\title{
Ricardo Galhardoni
}

Avaliação e tratamento de pacientes com dor facial atípica através da

Estimulação Magnética Transcraniana repetitiva

Tese apresentada à Faculdade de Medicina da Universidade de São Paulo para a obtenção do título de Doutor em Ciências - Programa de Neurologia

Orientadora: Prof. $^{\text {a }}$ Dra. Silvia Regina Dowgan Tesseroli de Siqueira

Coorientador: Prof. Dr. Daniel Ciampi Araujo de Andrade

São Paulo

2014 


\section{Ricardo Galhardoni}

Avaliação e tratamento de pacientes com dor facial atípica através da

Estimulação Magnética Transcraniana repetitiva

Tese apresentada à Faculdade de Medicina da Universidade de São Paulo para a obtenção do título de Doutor em Ciências - Programa de Neurologia

Orientadora: Prof. $^{\text {a }}$ Dra. Silvia Regina Dowgan Tesseroli de Siqueira

Coorientador: Prof. Dr. Daniel Ciampi Araujo de Andrade

São Paulo

2014 
Dados Internacionais de Catalogação na Publicação (CIP)

Preparada pela Biblioteca da

Faculdade de Medicina da Universidade de São Paulo

( $)$ reprodução autorizada pelo autor

Galhardoni, Ricardo

Avaliação e tratamento de pacientes com dor facial atípica através da estimulação magnética transcraniana repetitiva / Ricardo Galhardoni. -- São Paulo, 2014.

Tese(doutorado)--Faculdade de Medicina da Universidade de São Paulo. Programa de Neurologia.

Orientadora: Silvia Regina Dowgan Tesseroli de Siqueira

Coorientador: Daniel Ciampi Araujo de Andrade.

Descritores: 1.Dor facial/etiologia 2.Dor 3.Dor crônica 4.Estimulação magnética transcraniana 5.Avaliação 6.Terapêutica 7.Método duplo-cego

USP/FM/DBD-263/14 
A meus pais, Wilson e Luzia Galhardoni, e à minha irmã, Larissa Galhardoni, por tudo que sempre representaram em minha caminhada;

À Mariana Yumi Takahashi Puerta, por todo amor e carinho na luta diária. 
A Deus, por permitir que recomecemos diariamente a caminhada por um amanhã mais profícuo.

Aos pacientes que nos permitiram estudar sua condição a fim de que pudéssemos melhor entende-la para no futuro Ihes propiciar alívio.

À Prof. ${ }^{a}$ Silvia Regina Dowgan Tesseroli de Siqueira, por toda a orientação durante este trabalho, e por acreditar e investir na minha formação como profissional e pesquisador que hoje sou.

Ao Prof. Daniel Ciampi Araujo de Andrade, por aceitar a coorientação desde o início deste trabalho, e por toda sua parceria nas minhas empreitadas acadêmicas.

Ao Prof. Manoel Jacobsen Teixeira, pelo exemplo de dedicação à pesquisa e à boa prática clínica, e por permitir que este trabalho fosse desenvolvido no Centro de Dor do Departamento de Neurologia do HC/FMUSP.

Ao Prof. José Tadeu Tesseroli de Siqueira, pelos ensinamentos e discussões a respeito das dores orofaciais, e pelo auxílio na discussão a respeito de DFA.

A meus pais, Wilson e Luzia, pelas lutas diárias para que eu obtivesse uma boa formação desde o princípio.

À Mariana Y. T. Puerta, por toda a paciência, amor e carinho, e também por entender as ausências diárias para a realização e concretização deste trabalho. O seu apoio é sempre fundamental.

À Bruna L. R. Varotto, Mariana Y. T. Puerta e Rhianna B. Azeredo, pelas inúmeras avaliações dos doentes com DFA durante esta tese. 
Aos meus colegas do Centro de Dor, pela convivência no cotidiano de meus dias.

Aos colegas do Serviço de Estimulação Magnética Transcraniana do Instituto de Psiquiatria, pela convivência durante todo o período em que este trabalho foi desenvolvido.

À minha banca de qualificação, Dra. Rosana de Lima Pagano, Dra. Raquel Ruiz Chacon Martinez e Prof. Erich T. Fonoff, pelas valiosas contribuições neste trabalho.

À Thaís do Nascimento Figueira, pela paciência e auxílio nas questões burocráticas da pós-graduação.

À Alair Mariana dos Santos Silva, Elisabeth Lauritano, Elisete dos Santos Ferreira, Erli Vieira Soares Júnior, Ester Tomazzini, Maria Cecília Baptista Milani, Shirley Amaral Eugênio, Reiko Uchizono Simomura, Ruth Sousa Gomes, Sueli Martins, Valdenira dos Santos Silva, Vanessa Ramos Cardoso, Vera Lúcia Pereira, pelo apoio logístico dentro do Departamento de Neurologia HC/FMUSP.

À Sandra Aparecida de Lima Falcon, pelo apoio logístico no Laboratório de EMT do Instituto de Psiquiatria do HC/FMUSP.

Ao Conselho Nacional de Desenvolvimento Científico e Tecnológico (CNPq) pelo apoio financeiro através do processo no 141192/2012-7. 


\section{Normas adotadas:}

Esta tese está de acordo com as seguintes normas, em vigor no momento de sua publicação:

Referências: adaptado de International Committee of Medical Journals Editors (Vancouver).

Universidade de São Paulo. Faculdade de Medicina. Divisão de Biblioteca e Documentação. Guia de apresentação de dissertações, teses e monografias. Elaborado por Anneliese Carneiro da Cunha, Maria Julia de A. L. Freddi, Maria F. Crestana, Marinalva de Souza Aragão, Suely Campos Cardoso, Valéria Vilhena. 3ed. São Paulo: Divisão de Biblioteca e Documentação; 2011.

Abreviaturas dos títulos dos periódicos de acordo com List of Journals Indexed in Index Medicus. 
LISTA DE ABREVIATURAS 
A- $\beta$ : $\quad$ Fibras A-beta

A- $\delta$ : Fibras A-delta

$\%$ : Porcentagem

$\leq:$ Menor que

$\geq$ : $\quad$ Maior que

1B: Nível de evidência para estudos clínicos

2C+: Nível de evidência para estudos clínicos

5-HT: Serontonina

A/ $\mu$ s: Ampere por microssegundos

AMPA: Ácido alfa-amino-3-hidroxi-S-metil-4-isoxazole propiônico

ANOVA: Análise de Variância

ATM: Articulação temporomandibular

CDHCFMUSP: Centro de Dor do Hospital das Clínicas da Faculdade de Medicina

CGRP: Peptídeo geneticamente relacionado a calcitonina

$\mathrm{cm}$ : Centímetros

CPME: Corno posterior da medula espinal

d.C.: Depois de Cristo

D1 e D2: Receptor de dopamina

DFA: Dor facial atípica

DTM: Disfunção temporomandibular

EECM: Estimulação elétrica do córtex motor

EMG: Eletromiografia

EMT: Estimulação magnética transcraniana

EMTr: Estimulação magnética transcraniana repetitiva

EVA: Escala visual analógica

FIC: Facilitação intracortical

GABA A e B: Ácido gama aminobutírico

$\mathrm{Hz}: \quad$ Hertz

IASP: Associação Internacional para Estudos da Dor

IIC: Inibição intracortical 
M1: Córtex motor primário

ms: Milissegundos

NE: Noraepinefrina

NMDA: N-metil-D-aspartato

$\mathrm{NPH}$ : Neuralgia pós-herpética

PEM: Potencial evocado motor

PG: Prostaglandinas

R3: Reflexo de retirada 3

SI: Córtex sensitivo primário

SII: Córtex sensitivo secundário

SNC: Sistema nervoso central

SP: Substância P

$\mu: \quad$ Mi

$\mu \mathrm{V}$ : $\quad$ Microvolt 


\section{LISTA DE TABELAS}


TABELA 1 - ESTUDOS SOBRE AS PRINCIPAIS CARACTERÍSTICAS DA DFA.

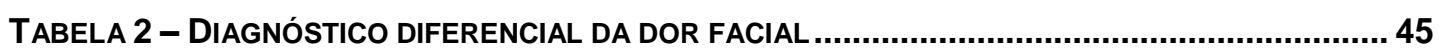

TABELA 3 - ESTUDOS SOBRE EMT-R EM DOR CRÔNICA INCLUINDO DOR FACIAL ….......................57

TABELA 4 - REVISÃO DOS PRINCIPAIS PARÂMETROS DE EXCITABILIDADE CORTICAL.....................61

TABela 5. Características Gerais de ACORDO COM O GRUPO DE DOENTES EM NúMEROS ABSOLUTOS (N), PORCENTAGENS (\%), MÉDIAS E DESVIO PADRÃO, E RESULTADOS DOS TESTES DE ASSOCIAÇÃO (P) ( $\mathrm{N}=29$ ).

TABELA 6 - MORBIDADES ASSOCIADAS DE ACORDO COM O GRUPO EM NÚMEROS ABSOLUTOS (N), PORCENTAGENS (\%) E RESULTADOS DOS TESTES DE ASSOCIAÇÃO (P) ( $\mathrm{N}=29$ ). 80

TABELA 7 - AUTO-AVALIAÇÃO DA SAÚDE DE ACORDO COM O GRUPO EM NÚMEROS ABSOLUTOS (N), PORCENTAGENS (\%) E RESULTADOS DOS TESTES DE ASSOCIAÇÃO (P) ( $\mathrm{N=29) \ldots ..........81}$

TABELA 8 - CARACTERÍSTICAS ODONTOLÓGICAS DE ACORDO COM O GRUPO EM NÚMEROS ABSOLUTOS (N), PORCENTAGENS (\%) E RESULTADOS DOS TESTES DE ASSOCIAÇÃO (P) $(\mathrm{N}=29)$ 82

TABELA 9 - CaRACTERísticas ÁlgiCAS de ACORDO COM O GRUPO EM NÚMEROS ABSOLUTOS (N), PORCENTAGENS (\%), MÉDIAS E DESVIO PADRÃO, E RESULTADOS DOS TESTES DE ASSOCIAÇÃO (P) ( $\mathrm{N}=29)$.

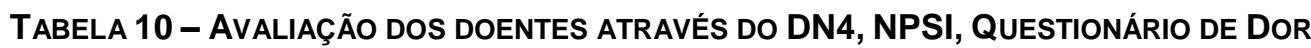
MCGILL, BPI E SF-36 DE ACORDO COM O GRUPO EM MÉDIAS E DESVIO PADRÃO, E RESULTADOS DOS TESTES DE ASSOCIAÇÃO (P) ( $N=29)$.

TABELA 11 - AVALIAÇÃo dOS DOENTES ATRAVÉS dO NPSI, QUESTIONÁRIO DE DOR MCGILL, BPI E SF-36 DE ACORDO COM O GRUPO EM MÉDIAS E DESVIO PADRÃO, E RESULTADOS DOS TESTES DE ASSOCIAÇÃO (P) NO TEMPO D7 ( $\mathrm{N}=29$ ).

TABELA 12 - AVALIAÇÃo dOS DOENTES ATRAVÉS do DN4, NPSI, QUESTIONÁRIO dE DOR MCGILL, BPI E SF-36 DE ACORDO COM O GRUPO EM MÉDIAS E DESVIO PADRÃO, E RESULTADOS DOS TESTES DE ASSOCIAÇÃO (P) NO TEMPO D21 ( $\mathrm{N}=29$ ). 90

TABELA 13 - AVALIAÇão dOS DOENTES ATRAVÉS DO DN4, NPSI, QUESTIONÁRIO DE DOR MCGILL, BPI E SF-36 DE ACORDO COM O GRUPO EM MÉDIAS E DESVIO PADRÃO, E RESULTADOS DOS TESTES DE ASSOCIAÇÃO (P) NO TEMPO D30 ( $N=29)$.

TABELA 14 - AVALIAÇÃo dos dOENTES atRAVÉS do DN4, NPSI, QUESTIONÁRIO de DOR

MCGILL, BPI E SF-36 DE ACORDO COM O GRUPO EM MÉDIAS E DESVIO PADRÃO, E RESULTADOS DOS TESTES DE ASSOCIAÇÃO (P) NO TEMPO D60 ( $N=29)$.

TABELA 15 - EXCITABILIDADE CORTICAL DOS DOENTES COM DFA COMPARADOS AOS CONTROLES SAUDÁVEIS EM MÉDIAS E DESVIO PADRÃO, E RESULTADOS DOS TESTES DE ASSOCIAÇÃO (P) $(\mathrm{N}=29)$ 138

TABELA 16 - EXCITABILIDADE CORTICAL DE ACORDO COM O GRUPO EM MÉDIAS E DESVIO PADRÃO, E RESULTADOS DOS TESTES DE ASSOCIAÇÃO (P) NO TEMPO D7 ( $N=29)$. 138 
TABELA 17 - EXCITABILIDADE CORTICAL DE ACORDO COM O GRUPO EM MÉDIAS E DESVIO PADRÃO, E RESULTADOS DOS TESTES DE ASSOCIAÇÃO (P) NO TEMPO D21 ( $\mathrm{N}=29$ ). 139

TABELA 18 - EXCITABILIDADE CORTICAL DE ACORDO COM O GRUPO EM MÉDIAS E DESVIO PADRÃO, E RESULTADOS DOS TESTES DE ASSOCIAÇÃO (P) NO TEMPO D30 ( $\mathrm{N}=29)$. 139

TABELA 19 - EXCITABILIDADE CORTICAL DE ACORDO COM O GRUPO EM MÉDIAS E DESVIO PADRÃO, E RESULTADOS DOS TESTES DE ASSOCIAÇÃO (P) NO TEMPO D60 ( $\mathrm{N}=29)$. 140

TABELA 20 - AVALIAÇÃO DOS DOENTES ATRAVÉS DO NPSI DE ACORDO COM O GRUPO E COM O PERÍODO EM MÉdIAS E DESVIO PADRÃO, E RESULTADOS DOS TESTES DE ASSOCIAÇÃo (P) $(\mathrm{N}=29)$ 140

TABELA 21 - AVALIAÇÃo DOS DOENTES ATRAVÉS DO BPI E DA EVA DE ACORDO COM O GRUPO E COM O PERÍODO EM MÉDIAS E DESVIO PADRÃO, E RESULTADOS DOS TESTES DE ASSOCIAÇÃO (P) $(\mathrm{N}=29)$. 141

TABela 22 - AVAliaÇÃo dos doentes ATRAVÉs do Questionário de Dor MCGiLl de ACORdo COM O GRUPO E COM O PERÍODO EM MÉDIAS E DESVIO PADRÃO, E RESULTADOS DOS TESTES DE ASSOCIAÇÃO (P) ( $N=29)$. 141

TABELA 23 - AVALIAÇÃO dOS DOENTES ATRAVÉS DO QUESTIONÁRIO DE QUALIDADE DE VIDA SF36 DE ACORDO COM O GRUPO E COM O PERÍODO EM MÉDIAS E DESVIO PADRÃO, E RESULTADOS DOS TESTES DE ASSOCIAÇÃO (P) ( $N=29$ ).

TABELA 24 - AVALIAÇÃO dOS DOENTES ATRAVÉS DO DN4 DE ACORDO COM O GRUPO E COM O PERÍODO EM MÉdIAS E DESVIO PADRÃO, E RESULTADOS DOS TESTES DE ASSOCIAÇÃo (P) $(\mathrm{N}=29)$. 


\section{LISTA DE FIGURAS}


FIGURA 1 - MECANISMO dE AÇÃO DA EMT

Figura 2 - CÁlCUlo do tAMANHO dA AMOSTRA SEGUNDO O PROGRAMA G*POWER 3.1...........64

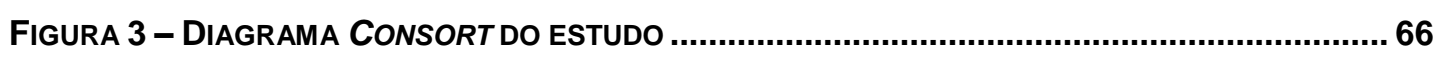

Figura 4 - Cronograma e Roteiro de AVALIAÇÃo duPLO-CEGA dos doENTES.....................69

FIGURA 5 - MOdELO dE MÁQUINA DE EMTR UTILIZADA NO ESTUDO. MAGPROX100 ${ }^{\circledR}$

(MAGVENTURE®-TONIKA EleKTRONIC, FARUM, DinAMARCA)...................................... 73

FIGURA 6 - DifERENÇAS ENTRE OS CAMPOS GERADOS PELA BOBINA CIRCULAR (ESQUEMA ESQUERDA) E BOBINA FIGURA DE OITO (ESQUEMA DIREITA). (MAGVENTURE® -

DiNAMARCA).

FIGURA 7 - AMPLITUDE DO LIMIAR MOTOR DE REPOUSO $(\mu \mathrm{V})$ DOS DOENTES COM DFA COMPARADOS AOS CONTROLES SAUDÁVEIS NO TEMPO BASAL E TESTE DE ASSOCIAÇÃO (P) $(\mathrm{N}=57)$.

FIGURA 8 - AMPLITUDE DO POTENCIAL EVOCADO MOTOR $(\mu \mathrm{V})$ DOS DOENTES COM DFA COMPARADOS AOS CONTROLES SAUDÁVEIS NO TEMPO BASAL E TESTE DE ASSOCIAÇÃo (P) $(\mathrm{N}=57)$.

FIGURA 9 - PORCENTAGEM DE INIBIÇÃO INTRACORTICAL DOS DOENTES COM DFA COMPARADOS AOS CONTROLES SAUDÁVEIS NO TEMPO BASAL E TESTE DE ASSOCIAÇÃO (P) ( $N=57)$......... 86

FigURA 10 - PORCENTAGEM DE FACILITAÇÃO INTRACORTICAL DOS DOENTES COM DFA COMPARADOS AOS CONTROLES SAUDÁVEIS NO TEMPO BASAL E TESTE DE ASSOCIAÇÃO (P) $(\mathrm{N}=57)$

FIGURA 11 - MÉdIA DE AMPLITUDE DO LIMIAR MOTOR DE REPOUSO $(\mu \mathrm{V})$ E INTERVALO DE CONFIANÇA DOS DOENTES COM DFA NO TEMPO D7 E TESTE DE ASSOCIAÇÃO (P) ( $N=29)$... 88

FIGURA 12 - MÉdIA DE AMPLITUDE DO POTENCIAL EVOCADO MOTOR $(\mu \mathrm{V})$ E INTERVALO DE CONFIANÇA DOS DOENTES COM DFA NO TEMPO D7 E TESTE DE ASSOCIAÇÃO (P) ( $N=29)$... 88

FIGURA 13 - MÉDIA DA PORCENTAGEM DE INIBIÇÃO INTRACORTICAL E INTERVALO DE CONFIANÇA DOS DOENTES COM DFA NO TEMPO D7 E TESTE DE ASSOCIAÇÃO (P) ( $\mathrm{N}=29$ ). 89

FIGURA 14 - MÉdIA DA PORCENTAGEM DE FACILITAÇÃO INTRACORTICAL E INTERVALO DE CONFIANÇA DOS DOENTES COM DFA NO TEMPO D7 E TESTE DE ASSOCIAÇÃO (P) ( $N=29)$... 89

FIGURA 15 - MÉdIA DE AMPLITUDE DO LIMIAR MOTOR DE REPOUSO $(\mu \mathrm{V})$ E INTERVALO DE CONFIANÇA DOS DOENTES COM DFA NO TEMPO D21 E TESTE DE ASSOCIAÇÃO (P) (N=29). 91

FIGURA 16 - MÉdIA DE AMPLITUDE DO POTENCIAL EVOCADO MOTOR $(\mu \mathrm{V})$ E INTERVALO DE CONFIANÇA DOS DOENTES COM DFA NO TEMPO D21 E TESTE DE ASSOCIAÇÃO (P) ( $\mathrm{N=29).} 91$

FiguRA 17 - MÉdIA DA PORCENTAGEM DE INIBIÇÃo INTRACORTICAL E INTERVALO DE CONFIANÇA DOS DOENTES COM DFA NO TEMPO D21 E TESTE DE ASSOCIAÇÃO ( $P$ ) ( $\mathrm{N}=29)$. 92

FIGURA 18 - MÉDIA DA PORCENTAGEM DE FACILITAÇÃO INTRACORTICAL E INTERVALO DE CONFIANÇA DOS DOENTES COM DFA NO TEMPO D21 E TESTE DE ASSOCIAÇÃO (P) ( $N=29) .92$

FIGURA 19 - MÉDIA DE AMPLITUDE DO LIMIAR MOTOR DE REPOUSO $(\mu \mathrm{V})$ E INTERVALO DE CONFIANÇA DOS DOENTES COM DFA NO TEMPO D30 E TESTE DE ASSOCIAÇÃO (P) ( $N=29) .94$ 
FIGURA 20 - MÉdIA DE AMPLITUDE DO POTENCIAL EVOCADO MOTOR $(\mu \mathrm{V})$ E INTERVALO DE CONFIANÇA DOS DOENTES COM DFA NO TEMPO D30 E TESTE DE ASSOCIAÇÃO (P) ( $\mathrm{N}=29) .95$

FIGURA 21 - MÉDIA DA PORCENTAGEM DE INIBIÇÃO INTRACORTICAL E INTERVALO DE CONFIANÇA DOS DOENTES COM DFA NO TEMPO D30 E TESTE DE ASSOCIAÇÃO (P) ( $\mathrm{N}=29)$. 95

FIGURA 22 - MÉdIA DA PORCENTAGEM DE FACILITAÇÃO INTRACORTICAL E INTERVALO DE CONFIANÇA DOS DOENTES COM DFA NO TEMPO D30 E TESTE DE ASSOCIAÇÃO (P) ( $\mathrm{N=29).} 96$

FIGURA 23 - MÉDIA DE AMPLITUDE DO LIMIAR MOTOR DE REPOUSO $(\mu \mathrm{V})$ E INTERVALO DE CONFIANÇA DOS DOENTES COM DFA NO TEMPO D60 E TESTE DE ASSOCIAÇÃO (P) ( $N=29) .98$

FIGURA 24 - MÉDIA DE AMPLITUDE DO POTENCIAL EVOCADO MOTOR $(\mu \mathrm{V})$ E INTERVALO DE CONFIANÇA DOS DOENTES COM DFA NO TEMPO D60 E TESTE DE ASSOCIAÇÃO (P) ( $\mathrm{N=29).} 98$

FIGURA 25 - MÉDIA DA PORCENTAGEM DE INIBIÇÃO INTRACORTICAL E INTERVALO DE CONFIANÇA DOS DOENTES COM DFA NO TEMPO D60 E TESTE DE ASSOCIAÇÃO (P) ( $\mathrm{N}=29)$. 99

FIGURA 26 - MÉdIA DA PORCENTAGEM DE FACILITAÇÃO INTRACORTICAL E INTERVALO DE CONFIANÇA DOS DOENTES COM DFA NO TEMPO D60 E TESTE DE ASSOCIAÇÃO (P) ( $\mathrm{N=29).} 99$

FIGURA 27 - ESCORE DO QUESTIONÁRIO DN-4 DOS DOENTES COM DFA DE ACORDO COM O GRUPO E COM O PERÍODO DE AVALIAÇÃO E INTERVALO DE CONFIANÇA, E RESULTADOS DOS TESTES DE ASSOCIAÇÃO (P) ( $\mathrm{N}=29)$. 100

FIGURA 28 - ESCORE DO QUESTIONÁRIO NPSI DOS DOENTES COM DFA DE ACORDO COM O GRUPO E COM O PERÍODO DE AVALIAÇÃO E INTERVALO DE CONFIANÇA, E RESULTADOS DOS TESTES DE ASSOCIAÇÃO (P) ( $N=29)$. 100

FIGURA 29 - ESCORE DA SUBESCALA INTERFERÊNCIA DO QUESTIONÁRIO BPI DOS DOENTES COM DFA DE ACORDO COM O GRUPO E COM O PERÍODO DE AVALIAÇÃO E INTERVALO DE CONFIANÇA, E RESULTADOS DOS TESTES DE ASSOCIAÇÃO (P) ( $\mathrm{N}=29$ ). 101

FIGURA 30 - ESCORE DA SUBESCALA (AFETIVO) DO QUESTIONÁRIO BREVE MCGILL DOS DOENTES COM DFA DE ACORDO COM O GRUPO E COM O PERÍODO DE AVALIAÇÃO E INTERVALO DE..102

FIGURA 31 - ESCORE DA SUBESCALA (SENSITIVO) DO QUESTIONÁRIO BREVE MCGILL DOS DOENTES COM DFA DE ACORDO COM O GRUPO E COM O PERÍODO DE AVALIAÇÃO E INTERVALO DE CONFIANÇA, E RESULTADOS DOS TESTES DE ASSOCIAÇÃO (P) ( $N=29)$....... 102

FIGURA 32 - ESCORE TOTAL DO QUESTIONÁRIO BREVE MCGILL DOS DOENTES COM DFA DE ACORDO COM O GRUPO E COM O PERÍODO DE AVALIAÇÃO E INTERVALO DE CONFIANÇA, E RESULTADOS DOS TESTES DE ASSOCIAÇÃO (P) ( $N=29$ ).

FIGURA 33 - ESCORE DO QUESTIONÁRIO DE QUALIDADE DE VIDA SF-36 DOMÍNIO ASPECTO FÍSICO, DOS DOENTES COM DFA E INTERVALO DE CONFIANÇA, E RESULTADOS DOS TESTES DE ASSOCIAÇÃO (P) ( $\mathrm{N}=29)$.

FIGURA 34 - ESCORE DO QUESTIONÁRIO DE QUALIDADE DE VIDA SF-36 DOMÍNIO ASPECTOS EMOCIONAIS, DOS DOENTES COM DFA E INTERVALO DE CONFIANÇA, E RESULTADOS DOS TESTES DE ASSOCIAÇÃO (P) ( $N=29)$. 
FIGURA 35 - ESCORE DO QUESTIONÁRIO DE QUALIDADE DE VIDA SF-36 DOMÍNIO ASPECTOS SOCIAIS, DOS DOENTES COM DFA E INTERVALO DE CONFIANÇA, E RESULTADOS DOS TESTES DE ASSOCIAÇÃO (P) ( $\mathrm{N}=29)$. 105

FIGURA 36 - ESCORE DO QUESTIONÁRIO DE QUALIDADE DE VIDA SF-36 DOMÍNIO CAPACIDADE FUNCIONAL, DOS DOENTES COM DFA E INTERVALO DE CONFIANÇA, E RESULTADOS DOS TESTES DE ASSOCIAÇÃO (P) ( $N=29)$. 105

FIGURA 37 - ESCORE DO QUESTIONÁRIO DE QUALIDADE DE VIDA SF-36 DOMÍNIO DOR, DOS DOENTES COM DFA E INTERVALO DE CONFIANÇA, E RESULTADOS DOS TESTES DE ASSOCIAÇÃO (P) ( $\mathrm{N}=29)$.

FIGURA 38 - ESCORE DO QUESTIONÁRIO DE QUALIDADE DE VIDA SF-36 DOMíNIO ESTADO GERAL DE SAÚDE, DOS DOENTES COM DFA E INTERVALO DE CONFIANÇA, E RESULTADOS DOS TESTES DE ASSOCIAÇÃO (P) ( $\mathrm{N}=29)$.

FIGURA 39 - ESCORE DO QUESTIONÁRIO DE QUALIDADE DE VIDA SF-36 DOMÍNIO SAÚDE MENTAL ASPECTO FÍSICO, DOS DOENTES COM DFA E INTERVALO DE CONFIANÇA, E RESULTADOS DOS TESTES DE ASSOCIAÇÃO (P) ( $\mathrm{N}=29)$. 107

FIGURA 40 - ESCORE DO QUESTIONÁRIO DE QUALIDADE DE VIDA SF-36 DOMÍNIO VITALIDADE, DOS DOENTES COM DFA E INTERVALO DE CONFIANÇA, E RESULTADOS DOS TESTES DE ASSOCIAÇÃO (P) ( $\mathrm{N}=29)$. 108

FIGURA 41 - ESCORE DA SUBESCALA DOR MAIS FRACA NAS ÚLTIMAS 24 HORAS DO QUESTIONÁRIO BPI DOS DOENTES COM DFA E INTERVALO DE CONFIANÇA, E RESULTADO DOS TESTES DE ASSOCIAÇÃO ( $P$ ) ( $N=29)$.

FIGURA 42 - ESCORE DA SUBESCALA DOR NO MOMENTO DA AVALIAÇÃO DO BPI DOS DOENTES COM DFA E INTERVALO DE CONFIANÇA, E RESULTADO DOS TESTES DE ASSOCIAÇÃO (P) $(\mathrm{N}=29)$. 109

FIGURA 43 - ESCORE DA SUBESCALA DOR MÉDIA NAS ÚLTIMAS 24 HORAS DO QUESTIONÁRIO BPI DOS DOENTES COM DFA E INTERVALO DE CONFIANÇA, E RESULTADO DOS TESTES DE ASSOCIAÇÃO ( $P$ ) ( $N=29)$.

FIGURA 44 - ESCORE DA SUBESCALA DOR MAIS FORTE NAS ÚLTIMAS 24 HORAS DO QUESTIONÁRIO BPI DOS DOENTES COM DFA E INTERVALO DE CONFIANÇA, E RESULTADO DOS TESTES DE ASSOCIAÇÃO (P) ( $\mathrm{N}=29)$.

Figura 45 - ANÁlise comparativa CuRVa de Kaplan-Meier entre os Grupos (Ativo E Placebo) PARA PORCENTAGEM DE MELHORA ESTIMADA EM 30\% DA EVA MÉdIA DOR TRATADA COM A EMTR $(\mathrm{N}=29)$. 
Galhardoni R. Avaliação e tratamento de pacientes com dor facial atípica através da estimulação magnética transcraniana repetitiva. [Tese]. São Paulo: Faculdade de Medicina, Universidade de São Paulo; 2014.

Dor facial atípica (DFA) é uma condição álgica crônica destacada pela etiopatogenia ainda desconhecida e pela característica rebelde aos tratamentos vigentes. O objetivo deste estudo é avaliar o limiar e padrões de excitabilidade cortical de doentes com DFA através de Estimulação Magnética Transcraniana (EMT) e compará-los a controles saudáveis, além de avaliar a eficácia terapêutica da EMT repetitiva (EMTr) ativa em doentes com DFA comparados à EMTr placebo. Durante o período de março de 2010 a dezembro de 2013 foram avaliados 29 doentes com DFA - os quais preencheram os critérios segundo a classificação proposta pela Associação Internacional de Cefaleia (2004) - e 28 controles saudáveis. A avaliação foi iniciada com a investigação da excitabilidade cortical bilateral. Em seguida, os doentes foram aleatorizados em dois grupos (ativo e placebo) para o tratamento com EMTr sobre o córtex motor primário na área de representação da face, esquematizado da seguinte forma: cinco sessões consecutivas para a fase de indução, e uma sessão semanal (pelo período de oito semanas) para a de manutenção; frequência excitatória de $10 \mathrm{~Hz}$; $80 \%$ do limiar motor de repouso; e 3000 pulsos no total por sessão. Os doentes foram avaliados quanto às características dolorosas no momento basal, no dia sete após a semana de indução, e nos dias 21, 30 e 60 para acompanhamento na fase de manutenção. Em todas as avaliações, utilizouse os seguintes questionários validados para a língua portuguesa: Inventário Breve de Dor; DN4, Inventário de Sintomas de Dor Neuropática, Questionário de descritores breve de dor McGill e Questionário de qualidade de vida SF-36. Os doentes do grupo ativo e placebo apresentaram excitabilidade cortical inicial diferenciada em relação aos controles nos padrões de inibição e facilitação intracortical $(p<0,001)$. Ao final da última avaliação, os doentes do grupo ativo apresentavam padrões de excitabilidade cortical mais próximos dos controles do que os doentes do grupo placebo, embora sem significância estatística. Não houve diferença entre os grupos ativo e placebo em todas as avaliações quanto às características de dor e de qualidade de vida, sendo que ambos apresentaram melhora da dor. Conclui-se que há diferenças neurofisiológicas entre os doentes com DFA e os controles, e que isso pode ser modificado através do EMTr. A ausência de efeito terapêutico da EMTr neste estudo indica que mais estudos utilizando-se outros parâmetros para a verificação da eficácia da EMTr na DFA são necessários.

Descritores: dor facial/etiologia; dor; dor crônica; estimulação magnética transcraniana; avaliação; terapêutica; método duplo-cego. 
Galhardoni R. Assessment and treatment of patients with atypical facial pain patients that underwent repetitive transcranial magnetic stimulation. [Thesis]. São Paulo: Faculdade de Medicina, Universidade de São Paulo; 2014

Atypical facial pain (AFP) is a chronic condition with unknown physiopathology and refractory characteristics to the gold standard treatment. The aim this study was to compare the patterns of cortical excitability between AFP and health subjects (HS), and to assess the analgesic effect of repetitive transcranial magnetic stimulation (rTMS) in AFP patients. Twenty-eight HS and 29 patients with AFP were included according to the IHS criteria (2004). Participants underwent a cortical excitability battery bilaterally in the primary motor cortex (M1) representation of the masseter muscle. They were then randomized into active and sham rTMS groups. rTMS was performed over the contralateral motor cortex in the representation area of the face daily for a week and weekly for eight weeks in a total of 13 sessions (5 induction and 8 maintenance sessions). All participants received $10 \mathrm{~Hz} \mathrm{rTMS}$, at $80 \%$ of the rest motor threshold (total of 3000 pulses per session). Sham rTMS was performed with an identical sham coil that emitted a similar sound to the active one. Patients were clinically assessed at baseline, after the induction phase (one week) and after 21,30 and 60 days after the beginning of the study. Evaluations included the following validated questionnaires to the Brazilian Portuguese language: brief pain inventory; DN-4; Neuropathic pain symptoms inventory, McGill pain questionnaire brief version and quality of life questionnaire SF36. At the baseline assessment, patients with AFP showed defective intracortical excitability inhibition and facilitation $(p<0.001)$ compared to HS. After the final evaluation, the patients from the active group had cortical excitability patterns closer to HS than the patients from the sham group, although there was no significant difference. There was no difference between patients that underwent active and sham rTMS about their pain characteristics and quality of life; both had pain improvement. In conclusion, there are neurophysiological differences between patients with AFP and HS, which could be modified with rTMS. The absence of a therapeutic effect of rTMS on pain in this study indicates the need of more research with other TMS parameters to check the efficacy of rTMS in AFP.

Descriptors: facial pain/etiology; pain; chronic pain; transcranial magnetic stimulation; evaluation; therapeutics and double-blind method. 
Lista de abreviaturas

Lista de tabelas

Lista de figuras

Resumo

Summary

1. INTRODUÇÃO.

2. OBJETIVOS

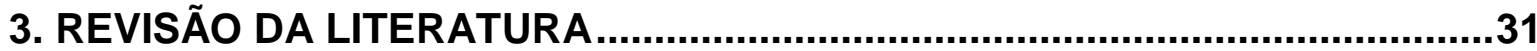

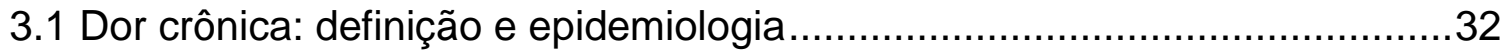

3.2 Dor crônica: fisiopatologia e neuroanatomia ....................................... 36

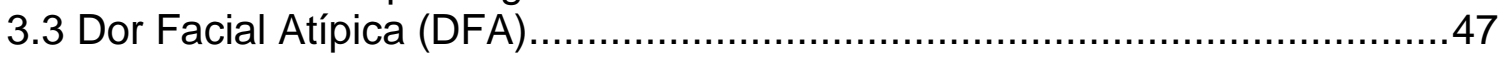

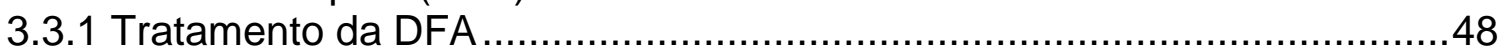

3.3.1.1 Tratamento farmacológico da DFA .............................................. 48

3.3.1.2 Medicamentos tópicos na DFA .......................................................49

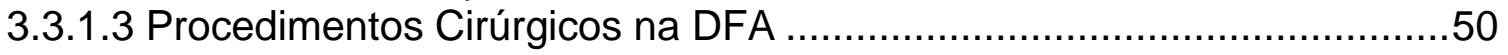

3.3.1.4 Estimulação magnética transcraniana (EMT) ..................................51

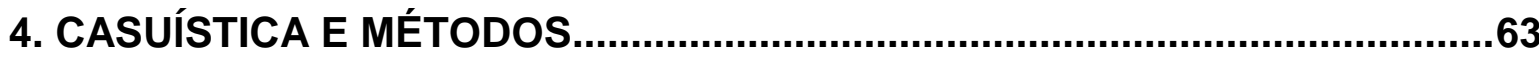

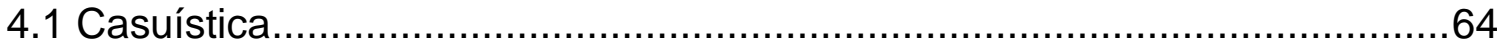

4.1.1 Critérios de Inclusão (grupos de estudo) (ANEXO 4) ............................67

4.1.2 Critérios de Inclusão (grupos de controle)

4.1.3 Critérios de Exclusão (grupo de estudo e controles)

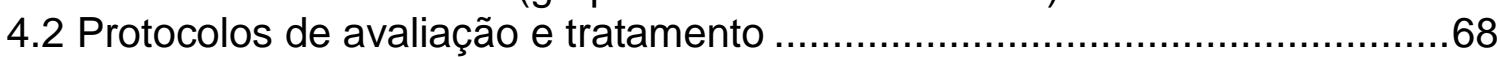

4.2.1 Instrumentos de avaliação.............................................................. 70

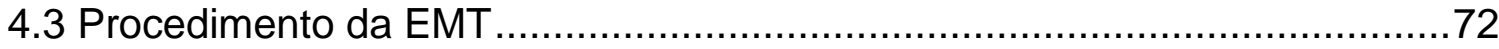

4.3.1 Medidas de excitabilidade cortical (grupos de estudo e controle) ..............72

4.3.2 Tratamento com EMTr (Ativo e Placebo) (grupos de estudo) ...................73

4.4 Análise dos dados ........................................................................... 75

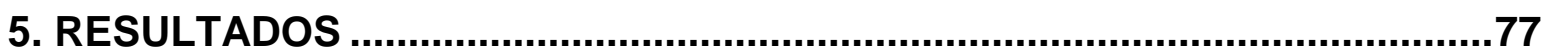

5.1. Avaliação inicial (Pré-tratamento) .................................................. 78

5.1.1 Características gerais dos doentes ............................................. 78

5.1.2 Características odontológicas, características álgicas e excitabilidade

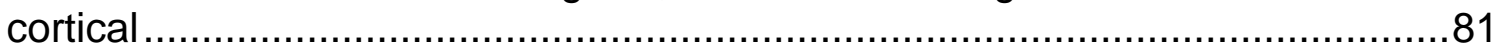

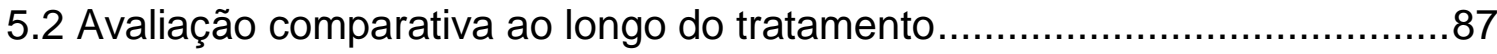

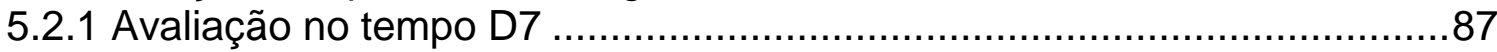

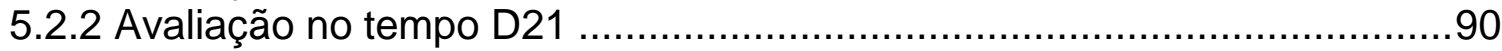

5.2.3 Avaliação no tempo D30 ................................................................93

5.2 .4 Avaliação no tempo D60 ...............................................................96

5.3 Comparação entre os tempos de tratamento e as avaliações......................99 


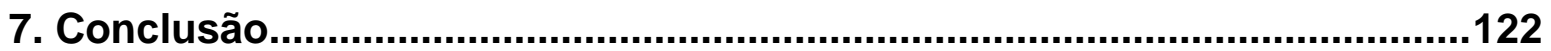

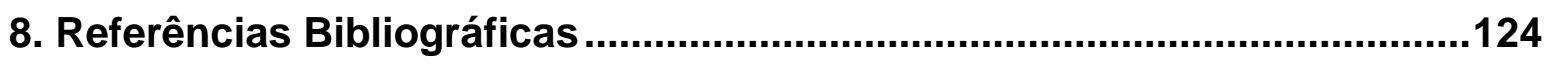

9. Apêndices

10. Anexos

Anexo 1: Aprovação do Comitê de Ética em Pesquisa do Hospital das

Clínicas da Faculdade de Medicina da Universidade de São Paulo.

Anexo 2: Registro do protocolo de estudo na plataforma ClinicalTrials.gov.....146

Anexo 3: Termo de Consentimento Livre e Esclarecido .................................147

Anexo 4: Critérios de Inclusão e exclusão grupo controle e doentes ...............149

Anexo 5: Ficha Clínica da Equipe de Dor Orofacial (EDOF) ...........................150

Anexo 6: Questionário de dor McGill resumido ..............................................154

Anexo 7: Inventário de Sintomas de Dor Neuropática (NPSI) ..........................155

Anexo 8: Questionário de Dor Neuropática 4 (DN-4) ....................................157

Anexo 9: Questionário de Qualidade de Vida SF-36 ....................................158

Anexo 10: Inventário Breve de Dor .........................................................161 
A dor crônica afeta entre $9 \%$ e $63,5 \%$ da população mundial e é uma condição altamente incapacitante, que interfere de forma marcante nas atividades do dia a dia, nos relacionamentos e na capacidade profissional e intelectual. Há elevados custos diretos e indiretos para o sistema de saúde, como perda de produtividade e aposentadoria precoce. Apesar dos avanços das pesquisas que visam a compreensão dos mecanismos fisiopatológicos com o intuito de desenvolver novas estratégias de tratamento, ainda há carência de modalidades terapêuticas realmente eficazes para o alívio da dor.

A dor facial atípica (DFA) é condição álgica crônica que se destaca pela etiopatogenia ainda desconhecida e por sua característica rebelde aos tratamentos vigentes. Descrita como dor facial profunda uni ou bilateral, sem respeitar o território trigeminal de inervação e de intensidade moderada ou grave, ela é também frequentemente associada às iatrogenias. Os medicamentos utilizados são antidepressivos tricíclicos, anticonvulsivantes e estabilizadores de membrana, porém raramente há completo desaparecimento dos sintomas. Nas últimas décadas, a Estimulação Magnética Transcraniana (EMT) foi desenvolvida como ferramenta de tratamento em neuropsiquiatria, sendo muito utilizada, com bons resultados, em doenças psiquiátricas. Trata-se de uma técnica não invasiva baseada no princípio de Faraday que serve como ferramenta neurofisiológica para mensuração e modulação da excitabilidade cortical. Devido à carência de estudos em dor facial, foi realizado ensaio controlado duplo cego para verificar aspectos fisiopatológicos e terapêuticos da EMT na DFA. 
O presente estudo teve como objetivos:

1) Avaliar o limiar e os padrões de excitabilidade cortical de doentes com DFA através da EMT comparados a controles saudáveis, comparando os limiares e os padrões encontrados com os resultados após EMT repetitiva (EMTr) (ativo e placebo);

2) Avaliar a eficácia terapêutica da EMTr no córtex motor primário em doentes com DFA comparada à EMTr placebo. 


\subsection{Dor crônica: definição e epidemiologia}

Dor é uma experiência sensitiva e emocional desagradável, associada à lesão real, em potencial ou descrita em tais termos (International Association for the Study of Pain, 1986). Pode ser compreendida como sofrimento e, desde os primórdios, provoca esforços humanos na busca do alívio. Por conta disso, a compreensão fisiopatológica e de mecanismos adjacentes é essencial para seu tratamento. A dor aguda - com duração de até três meses - tem função biológica de manutenção da vida. Após esse período, ela passa a ser crônica. Essa geralmente não tem função biológica, podendo causar incapacidade temporária ou permanente dos indivíduos (Twaddle, Cooke, 2006; Von Roenn, Paice et al., 2006; Cruccu, Sommer et al., 2010; Teixeira, Siqueira et al., 2012a). Sua subjetividade dificulta o diagnóstico e o tratamento.

Dor crônica é prevalente, afetando cerca de $10,1 \%$ a $55,2 \%$ da população anglo-saxônica (Harstall, Ospina, 2003) e gerando altos custos diretos e indiretos. Cerca de $75 \%$ a $80 \%$ dos atendimentos em saúde são motivados pela dor e aproximadamente $30 \%$ a $40 \%$ dos indivíduos com dor crônica utilizam cinco vezes mais o sistema de saúde do que a média geral de outros usuários. Na população com mais de 60 anos, há prevalência de dor crônica de $51,4 \%$, a qual diminui após os 75 anos (Teixeira, Siqueira et al., 2012a). Entre os doentes internados, a frequência de dor é de $45 \%$ a $80 \%$, tendo como causas mais comuns as epigastralgias e outras dores abdominais, dor à micção, cefaleias, artralgias, dores nos membros e dor torácica (Teixeira, Kraychete et al., 2006). As afecções do aparelho 
locomotor afetam cerca de $42,8 \%$ da população, e são seguidas pelas afecções neuropáticas $(31,2 \%)$. Teixeira et al. (2006) observaram que a síndrome fibromiálgica, a dor miofascial e a dor abdominal apresentam como média etária 49,6 anos, sendo que a neuralgia pós-herpética, a dor no coto amputado e a dor pós-acidente vascular encefálico são mais comuns em indivíduos mais idosos. As dores crônicas são mais prevalentes entre os indivíduos do sexo feminino e são localizadas principalmente na região dorsal e em membros inferiores. Elas provocam diminuição da funcionalidade física, aumento da insônia, depressão, dificuldades ao caminhar e, consequente, diminuição da qualidade de vida (Teixeira, Siqueira et al., 2012a). Dentre os problemas econômicos aventados, destacam-se: redução da jornada diária de trabalho; absenteísmo; processos de aposentadoria e pensões precoces.

Os estudos epidemiológicos sobre a dor no Brasil ainda são escassos e em geral centrados em condições ou locais específicos. Em Salvador, a dor crônica foi observada em $41,4 \%$ da população, sendo que $48,4 \%$ eram do sexo feminino e 16,3\% tinham como queixa principal a lombalgia (Sa, Baptista et al., 2008). Este resultado corrobora a revisão realizada por Teixeira et al. (Teixeira, Kraychete et al. 2006) na qual é relatada dor lombar de $8 \%$ a $45 \%$ da população brasileira adulta, enquanto que na população americana essa prevalência é de $26,3 \%$. Ferreira et al. (2011) também relataram que intensidade da dor, sexo feminino, depressão, ansiedade ou outros transtornos psiquiátricos, morbidades associadas, baixo nível de escolaridade, alto índice de massa corpórea e idade avançada foram 
preditores de dor crônica. A diferença cultural também é um fator que pode influenciar a prevalência de dor e o comportamento associado (Fillingim, 2012).

No segmento cefálico, a dor afeta cerca de $70 \%$ dos indivíduos, sendo que em $10 \%$ ela é crônica. Aproximadamente $90 \%$ dos homens e $95 \%$ das mulheres apresentam cefaleia ao menos uma vez ao ano. Ela é observada em $3 \%$ a $8 \%$ das crianças com 3 anos, $19 \%$ das crianças com 5 anos e em $37 \%$ a $51,5 \%$ das crianças entre 7 a 15 anos de idade. Dentre as cefaleias funcionais, as mais prevalentes são a migrânea (afeta $25 \%$ dos indivíduos, sendo $20 \%$ do sexo feminino) e as do tipo "tensão". Ao menos um episódio de cefaleia do tipo tensão foi descrito por $79 \%$ dos indivíduos, sendo que $20 \%$ a 30\% deles têm dor crônica (Teixeira, Siqueira et al., 2012a).

Dor de dente é uma mais comuns e conhecidas dores da humanidade, observada em $40 \%$ da população mundial, sendo que a dor facial afeta $12 \%$, e a cefaleia, 26\% (Siqueira, 2012). Dados semelhantes são observados nos Estados Unidos e no Brasil, havendo prevalência (12\%) de odontalgia entre adolescentes, com incapacitação média de 4,5 dias (Siqueira, 2012). A frequência de traumatismo dentário na população brasileira é de $8 \%$ aos 9 anos de idade, e de $13,6 \%$ aos 12 anos, chegando a $16,1 \%$ aos 14 anos (Siqueira, 2012).

A segunda causa mais comum de dor facial é a disfunção temporomandibular (DTM) (Zakrzewska, 2009). É mais comum em jovens entre 32,6 a 39 anos, e pode estar relacionada ao uso de próteses. Dor ao 
abrir a boca (5\%), ruído articular (24\%) e dor em masseter (39\%) podem estar presentes (Gonçalves, Dal Fabbro et al., 2010).

A cervicalgia afeta entre 9,5 e $35 \%$ dos indivíduos. Ela é mais comum em mulheres $(13,5 \%)$ do que em homens $(9,5 \%)$. Contudo, apenas $4 \%$ das mulheres a apresenta antes dos 30 anos de idade (Teixeira, Siqueira et al., 2012a)

Em 1997, a Neuralgia pós-herpética (NPH) foi prevalente em 12,4\% dos pacientes atendidos na triagem do Centro de Dor do Hospital das Clínicas da Faculdade de Medicina da USP (CDHCFMUSP). Sua incidência é de 4,8 casos a cada cem mil habitantes na terceira idade, e de 1,3 casos nas demais faixas etárias (Teixeira, Okada, 1999; Teixeira, Siqueira et al., 2012a). A NHP pode ser acompanhada de disestesia anterior à erupção (entre $20 \%$ e $40 \%$ ), que se mantém em $9 \%$ e $25 \%$ dos casos, tornando-se crônica em $10 \%$, principalmente em idosos (47,5\% a $70 \%$ ) (Teixeira, Okada, 1999). Ela afeta a face em $17 \%$ a $25 \%$ dos casos (desses, $88 \%$ a $94 \%$ são no nervo trigêmeo, principalmente no ramo oftálmico) (Teixeira, Okada, 1999; Alvarez, de Siqueira et al., 2007).

A neuralgia idiopática do trigêmeo apresenta prevalência de 155 indivíduos a cada um milhão de habitantes nos Estados Unidos e na França. Sua incidência é de 4,3 a cada cem mil habitantes, sendo mais comum nas mulheres. Ela apresenta padrão familiar em 2,6\% a 6\% dos casos (Siqueira, Teixeira, 2012a). A síndrome da ardência bucal afeta de $0,7 \%$ a $18 \%$ da população e é mais comum em mulheres pós-menopausa (Nasri e Siqueira 2012). 
A classificação de dor crônica baseia-se em geral nos mecanismos fisiopatológicos. A mais comum é a dor de origem musculoesquelética, seguida da dor neuropática. Essa decorre de lesão nervosa periférica e/ou central e anormalidades no trato espinotalâmico, a exemplo da dor póstrauma do nervo alveolar inferior ou polineuropatia diabética. A dor nociceptiva está associada à inflamação e pode ser exemplificada pelas osteoartrites, pulpites e angina isquêmica. Dor de caráter disfuncional é aquela que não apresenta evidência de lesão somestésica, como a síndrome fibromiálgica, a síndrome do intestino irritado e as cefaleias. A DFA pode ser incluída nesse grupo. Por fim, a dor visceral está relacionada a órgãos como fígado, coração e pulmão, sendo comumente irradiada, difusa e difícil de ser identificada (Dworkin, Backonja et al., 2003; Giamberardino, Affaitati et al., 2006; Zhang e Baccei, 2008; Teixeira, Siqueira et al., 2012b).

\subsection{Dor crônica: fisiopatologia e neuroanatomia}

O processo álgico inicia-se pela transdução de estímulos mecânicos, térmicos ou químicos em potenciais de ação nos aferentes primários mielínicos do tipo A-delta $(A-\delta)$ e amielínicos $C$ que acessam o sistema nervoso central (SNC). A porção terminal periférica responsável pela transdução nos aferentes primários é denominada receptor (Sessle, 2005). Enquanto as fibras A-beta $(A-\beta)$ possuem receptores de baixo limiar e são ativadas por estímulos mecânicos não nociceptivos ou por movimentos 
(Sessle, 2005), as fibras A- $\delta$ e C possuem terminações livres nos tecidos periféricos e são ativadas por nocicepção (Tracey, Mantyh, 2007; Teixeira, 2009; Gold, Gebhart, 2012). Sabe-se que os tecidos orofaciais, como dentes, pele da face, articulação temporomandibular (ATM) e músculos mastigatórios são inervados pelos ramos do $\mathrm{V}$ par craniano, o nervo trigêmeo.

Tão logo há o fenômeno álgico, inicia-se a sensibilização periférica ou hiperalgesia primária, caracterizada pela diminuição de limiar nos receptores nociceptivos (Johanek, Shim et al., 2006). Diversos mediadores, como a substância $\mathrm{P}(\mathrm{SP})$, o peptídeo geneticamente relacionado à calcitonina (CGRP), as prostaglandinas (PG), interleucinas, bradicinina e serotonina participam desse processo. No SNC, há facilitação da passagem do estímulo do aferente primário ao segundo neurônio do corno posterior da medula espinal (CPME), o que caracteriza sensibilização central ou hiperalgesia secundária, promovendo desinibição e redução de limiar através de mecanismos de neuroplasticidade.

A sensibilização central corresponde a um estado de hiperexcitabilidade patológica dos neurônios nociceptivos centrais que causam não somente respostas aumentadas a estímulos normalmente nociceptivos, como também respostas patológicas a estímulos não dolorosos (Sessle, 2005), sendo dependente da hiperatividade de fibras C principalmente nos estágios iniciais. Em condições normais, aminoácidos excitatórios como o glutamato ativam receptores do tipo ácido alfa-amino-3hidroxi-S-metil-4-isoxazole propiônico (AMPA)/kainato. Em casos de 
ativação prolongada de neurônios do tipo $\mathrm{C}$, a liberação exagerada de glutamato libera o íon magnésio que "bloqueia" os receptores NMDA. Estes causam grande entrada de cálcio na célula, o que altera a expressão gênica e a atividade elétrica, acarretando em modificações estruturais a longo prazo (Lavigne, Woda et al., 2005; Sessle, 2005; Johanek, Shim et al., 2006; Todd, 2006; Teixeira, 2009; Teixeira, Siqueira et al., 2012b).

As vias aferentes convergem para o complexo nuclear trigeminal que é formado pelos núcleos principal, mesencefálico e trato espinal. $O$ trato espinal divide-se em subnúcleos oral, interpolar e caudal, sendo que o último tem relação de continuidade com o corno posterior da medula espinal (Woda, 2003). A organização nociceptiva no subnúcleo caudal apresenta projeções axônicas ascendentes (Todd, 2006). Neurônios nociceptivos da parte rostral do subnúcleo oral promovem alterações neuroplásticas definitivas nas informações nociceptivas características da dor crônica, sendo que a tratotomia do núcleo caudal em humanos, gatos e ratos não é capaz de aliviar completamente a dor de origem bucal, necessitando de intervenção no subnúcleo oral (Woda, 2003).

O núcleo do trato espinal projeta-se para o tálamo e posteriormente para o córtex somatossensitivo. As transmissões pré-sinápticas nociceptivas dependem do glutamato, da substância P e do aspartato, e as transmissões pós-sinápticas dependem das proteínas G, N-metil-D-aspartato (NMDA) e (AMPA) (Lavigne, Woda et al., 2005; Wood, 2006; Teixeira 2009) .

As fibras do trato espinotalâmico originam-se nas lâminas I, IV, V, VI, VII e VIII do corno posterior da medula espinal, sendo que aproximadamente 
dois terços dessas fibras são nociceptivas rápidas ou lentas (Todd, 2006; Teixeira, 2009). Essas fibras fazem sinapse com neurônios do tálamo nas seguintes regiões: complexo ventrobasal, grupamento nuclear posterior, núcleos intralaminares e núcleo submédio. As fibras espinorreticulotalâmicas projetam-se para os núcleos centro-mediano, centro-lateral e parafascicular do tálamo e para o componente magnocelular do corpo geniculado medial. Não há consenso em relação às projeções diretas dos aferentes nociceptivos no núcleo centro-mediano (Teixeira, 2009). A organização somatotópica é bem definida no complexo ventrobasal do tálamo, e seus neurônios projetam-se aos córtices sensitivos SI e SII. Essas projeções de neurônios exercem função integradora entre as atividades motora, sensitiva, psicocomportamental e neurovegetativa (Teixeira, 2009; Lorenz, Hauck, 2012). As vias espinotalâmica e cortical ventroposterolateral estão relacionadas ao componente discriminativo da sensação de dor (localização, duração, natureza), bem como à temporalidade.

A informação dolorosa é processada em várias outras regiões do encéfalo, incluindo o córtex cingular anterior, o córtex frontal, o cerebelo (Garcia-Larrea, 2012), o corpo estriado, o putamem e a amígdala (Lavigne, Woda et al., 2005; Teixeira, 2009). A formação reticular tem projeções para o tálamo medial e para o trato mesencefálico, sendo que essas seguem para áreas parabranquiais e estruturas límbicas, como hipotálamo, amígdala, giro do cíngulo anterior e áreas corticais frontais. As regiões límbicas e paralímbicas estão relacionadas ao componente afetivo-emocional (Craig, 2002; Teixeira, 2009). 
A ínsula apresenta papel importante na função neurovegetativa e na discriminação térmica da dor em relação ao calor e ao frio. Há um aumento expressivo da atividade no lobo frontal tanto no estímulo doloroso cutâneo quanto no visceral (Teixeira, 2009; Craig, 2011), bem como no córtex orbitário, no núcleo acumbente e no tronco encefálico.

\subsection{Dor Facial Atípica (DFA)}

A Dor Facial Atípica (DFA), descrita a primeira vez por Fraizer e Russell em 1924, é caracterizada por dor difusa, uni ou bilateral, mais frequentemente na maxila (Ram, Teruel et al., 2009), sem causa aparente e sem preencher critérios de neuralgia craniana clássica ou outro diagnóstico orofacial. Geralmente, é latejante e profunda, não acompanhada de anormalidades neurológicas (Lavigne, Woda et al., 2005). Afeta $10 \%$ da população adulta e 50\% da população idosa (Agostoni, Frigerio et al., 2005). Pelo fato de ter uma etiologia controversa e mecanismos fisiopatológicos ainda obscuros, não há consenso em sua classificação, o que acaba levando à necessidade de exclusão das demais patologias para que seja feito o diagnóstico (Woda e Pionchon, 1999; Teixeira, Bor Seng Shu et al., 2000; Nóbrega, Siqueira et al., 2007; Siqueira, Marcolin et al., 2010).

Em sua classificação criada por Merskey e Bogduk em 1994, a Associação Internacional para Estudos da Dor (IASP) aloca a DFA no grupo IV, que inclui lesões de ouvido, nariz e cavidade oral (item IV-5, código 034.X8b). Nessa classificação, a DFA está associada à 
hipertermosensibilidade, sendo que o procedimento odontológico prévio é um dos critérios para o diagnóstico.

A Sociedade Internacional de Cefaleias denomina a DFA como dor facial idiopática, caracterizada por dor persistente na face e/ou região oral, mal localizada, que não respeita o território de distribuição nervosa, com diferentes apresentações, recorrente ao longo do dia por mais de duas horas por um período superior a três meses, e na ausência de déficit neurológico clínico (IHS 2013).

Os estudos nacionais e internacionais demonstram que a prevalência da DFA é maior em mulheres do que homens, sendo que, geralmente, ela é unilateral e predominantemente à esquerda (Pfaffenrath, Rath et al., 1993; Garcia, Okada et al., 1999; Teixeira, Bor Seng Shu et al., 2000; Nóbrega, 2002; Prado 2002; Siqueira, Ching et al., 2004; Nóbrega, Siqueira et al., 2007; Ram, Teruel et al., 2009). Os descritores mais comuns são queimação (Pfaffenrath, Rath et al., 1993; Vickers, Cousins et al., 1998; Teixeira, Bor Seng Shu et al., 2000; Nóbrega, Siqueira et al., 2007;) e latejamento (Pfaffenrath, Rath et al., 1993; Vickers, Cousins et al., 1998; Teixeira, Bor Seng Shu et al., 2000; List, Leijon et al., 2007; Nóbrega, Siqueira et al., 2007; Takenoshita, Sato et al., 2010). A média de duração varia de 24 a 161,4 meses (Hagelberg, Forssell et al., 2003; Wirz, Wartenberg et al., 2005; Nóbrega, Siqueira et al., 2007) e a média de idade dos doentes varia de 31,9 a 57,2 anos (Nóbrega, 2002; Prado, 2002; Wirz, Wartenberg et al., 2005; Zagury, Eliav et al., 2011). Há uma maior incidência de dor na região maxilar do que na região mandibular, bem como a predominância de queixas nos 
dentes pré-molares (List, Leijon et al., 2007; Zagury, Eliav et al., 2011) (Tabela 1). 
Tabela 1 - Estudos sobre as principais características da DFA

\begin{tabular}{|c|c|c|c|c|c|}
\hline Autor & $\begin{array}{c}\text { Número } \\
\text { de } \\
\text { sujeitos }\end{array}$ & $\begin{array}{c}\text { Idade } \\
\text { (média em } \\
\text { anos) }\end{array}$ & Razão homem/mulher & Localização da dor (lado da face) & $\begin{array}{c}\text { Tempo de } \\
\text { dor (em meses) }\end{array}$ \\
\hline Pfaffenrath et al., 1993 & 35 & 53.2 & $4 / 31$ & $\begin{array}{l}60 \% \text { unilateral; } 23 \text { pacientes lado } \\
\text { direito } \\
11 \text { pacientes lado esquerdo }\end{array}$ & 84 \\
\hline Vickers et al., 1998 & 50 & 51 & $16 / 34$ & n.d. & 57 \\
\hline Garcia et al., 1999 & 31 & n.d. & $10 / 21$ & n.d. & n.d. \\
\hline Teixeira et al., 2000 & 57 & 51 & $18 / 39$ & $\begin{array}{c}\text { Bilateral } 14,2 \% \\
\text { Esquerdo } 39 \% \\
\text { Direito } 47 \%\end{array}$ & 61,8 \\
\hline Hagelberg et al., 2003 & 8 & 56 & $8 / 0$ & $\begin{array}{c}3 \text { pacientes lado direito } \\
2 \text { pacientes lado esquerdo } \\
2 \text { pacientes bilateral }\end{array}$ & 156 \\
\hline Siqueira et al., 2004 & 26 & n.d. & $6 / 20$ & $\begin{array}{c}11 \text { lado direito; } \\
8 \text { lado esquerdo } \\
7 \text { bilateral }\end{array}$ & 31.2 \\
\hline Wirz et al., 2005 & 985 & n.d. & $66,8 \%$ mulheres & n.d. & 31.9 \\
\hline Nobrega et al., 2007 & 51 & 54,4 & $24 / 27$ & $\begin{array}{l}\text { Lado esquerdo } 9 \\
\text { Lado direito } 6 \\
\text { Bilateral } 6\end{array}$ & 161.4 \\
\hline List et al., 2007 & 46 & 56 & $11 / 24$ & n.d. & 91 \\
\hline Ram et al., 2009 & 64 & 55,4 & $20 / 44$ & $\begin{array}{l}\text { Lado esquerdo } 32 \\
\text { Lado direito } 19 \\
\text { Bilateral } 13\end{array}$ & 33 \\
\hline Takenoshita et al., 2010 & 37 & 45,7 & $7 / 30$ & $\begin{array}{c}\text { Gengiva } 33,3 \% \\
\text { Face } 36,1 \%\end{array}$ & 31,9 \\
\hline Pigg et al., 2011 & 20 & 52 & $2 / 18$ & $\begin{array}{c}\text { Maxila 12/18* } \\
\text { Região posterior } 13 / 16^{*}\end{array}$ & 51 \\
\hline Zagury et al., 2011 & 39 & 57,2 & $77 \%$ mulheres & $\begin{array}{l}59 \% \text { pré-molar; } \\
22 \% \text { molar; } \\
14 \% \text { incisivo }\end{array}$ & n.d. \\
\hline
\end{tabular}

Legenda: n.d. não descrito, *um paciente apresentava dor bilateral. 
O diagnóstico diferencial da DFA é complexo, por depender da exclusão de outras doenças. Dor facial sintomática poderia ser caracterizada como aquela que preenche critérios da DFA mas tem outra causa. Mais da metade de uma amostra estudada $(51,2 \%)$ foi considerada dor facial sintomática (Nóbrega, 2002). Os diagnósticos foram: Síndrome de Wallemberg; neuropatia por processo expansivo intracraniano; neuropatia iatrogênica; dor decorrente de carcinoma de língua, síndrome da ardência bucal; fibromialgia e DTM. A Tabela 2 apresenta os principais diagnósticos diferenciais da DFA (Agostoni et al., 2005).

Em $30 \%$ dos casos, a DFA apresenta como fator de piora a mastigação, seguida da emoção (25\%) e o consumo de alimentos gelados (25\%). Morbidades associadas são: migrânea (25\%), Diabetes mellitus tipo II (15\%) e acidente vascular encefálico (5\%). Os indivíduos procuram de dois a quinze profissionais até serem diagnosticados (Prado, 2002). Edentulismo total é frequentemente observado (40\%), sendo que $35 \%$ são edêntulos parciais (Nóbrega, 2002). 
Tabela 2 - Diagnóstico diferencial da dor facial

\begin{tabular}{|c|c|c|c|c|c|c|}
\hline Dor facial & Localização & Qualidade & Intensidade & Duração & $\begin{array}{c}\text { Fatores } \\
\text { Agravantes }\end{array}$ & $\begin{array}{c}\text { Outras } \\
\text { características }\end{array}$ \\
\hline Neuralgia do Trigêmeo & $\begin{array}{l}\text { Respeita território de inervação } \\
\text { trigeminal geralmente unilateral }\end{array}$ & $\begin{array}{l}\text { Lancinante, } \\
\text { pontada, } \\
\text { queimação, } \\
\text { choque } \\
\text { elétrico }\end{array}$ & Grave & $\begin{array}{l}\text { Segundos a } \\
\text { minutos }\end{array}$ & $\begin{array}{l}\text { Tocar ou lavar a } \\
\text { face, comer, } \\
\text { mastigar, sorrir, } \\
\text { falar, assoar o } \\
\text { nariz, escovar os } \\
\text { dentes, bocejar, } \\
\text { barbear }\end{array}$ & - \\
\hline $\begin{array}{l}\text { Neuralgia pós-herpética } \\
\text { trigeminal }\end{array}$ & $\begin{array}{l}\text { Usualmente ramo oftálmico ou } \\
\text { maxilar do } 5^{\circ} \text { par craniano, unilateral }\end{array}$ & $\begin{array}{l}\text { Dolorida, } \\
\text { queimação, } \\
\text { punhalada }\end{array}$ & Grave & Constante & $\begin{array}{l}\text { Contato, } \\
\text { movimento }\end{array}$ & \\
\hline Dor facial atípica & $\begin{array}{l}\text { Em um lado da face, asa do nariz ou } \\
\text { lateral, queixo, mandíbula, pescoço; } \\
\text { mal localizada }\end{array}$ & $\begin{array}{l}\text { Profunda, } \\
\text { dolorida, } \\
\text { Latejante, } \\
\text { queimação }\end{array}$ & $\begin{array}{l}\text { Moderada a } \\
\text { grave }\end{array}$ & Constante & 然 & 然 \\
\hline Disfunção Temporomandibular & $\begin{array}{l}\text { Mandíbula, região pré-auricular, } \\
\text { têmporas, fronte, face }\end{array}$ & $\begin{array}{l}\text { Apunhalada, } \\
\text { aborrecida }\end{array}$ & Moderada & $\begin{array}{l}\text { Minutos a } \\
\text { horas }\end{array}$ & $\begin{array}{l}\text { Palpação da } \\
\text { articulação } \\
\text { mandibular e dos } \\
\text { músculos da } \\
\text { mastigação }\end{array}$ & $\begin{array}{l}\text { Abertura incompleta } \\
\text { da mandíbula, } \\
\text { movimentos laterais }\end{array}$ \\
\hline Síndrome de Tolosa-Hunt & Principalmente retro-orbital, unilateral & Dolorida & Grave & Constante & - & $\begin{array}{l}\text { Oftalmoplegia, perda } \\
\text { sensitiva acima da } \\
\text { fronte, ptose, pupilas } \\
\text { normalmente } \\
\text { normais }\end{array}$ \\
\hline $\begin{array}{c}\text { Síndrome paratrigeminal de } \\
\text { Raeder }\end{array}$ & Frontotemporal e maxila, unilateral & $\begin{array}{l}\text { Profunda, } \\
\text { lancinante }\end{array}$ & Grave & Constante & - & Ptose, miose \\
\hline
\end{tabular}


Continuação da página anterior

Tabela 2 - Diagnóstico diferencial da dor facial

\begin{tabular}{|c|c|c|c|c|c|c|}
\hline Dor facial & Localização & Qualidade & Intensidade & Duração & $\begin{array}{c}\text { Fatores } \\
\text { Agravantes }\end{array}$ & $\begin{array}{c}\text { Outras } \\
\text { características }\end{array}$ \\
\hline Carotidinia & $\begin{array}{l}\text { Face, orelha, mandíbula, dentes, } \\
\text { região acima do pescoço, unilateral }\end{array}$ & Latejante & Moderada & Constante & $\begin{array}{l}\text { Compressão } \\
\text { comum da } \\
\text { carótida }\end{array}$ & \\
\hline Cefaleia tipo tensão & Frontotemporal ou parietal, bilateral & $\begin{array}{l}\text { Pressão, } \\
\text { aperto }\end{array}$ & $\begin{array}{l}\text { Leve a } \\
\text { moderada }\end{array}$ & $\begin{array}{l}\text { Minutos a } \\
\text { dias }\end{array}$ & - & \\
\hline Migrânea & $\begin{array}{l}\text { Frontotemporal, orbital, usualmente } \\
\text { bilateral }\end{array}$ & $\begin{array}{l}\text { Pressão, } \\
\text { aperto }\end{array}$ & $\begin{array}{l}\text { Moderada a } \\
\text { grave }\end{array}$ & Horas & Atividade física & Migrânea com aura \\
\hline Pulpite & $\begin{array}{c}\text { Dente, outras partes da face, } \\
\text { frontotemporal, não muito bem } \\
\text { localizada }\end{array}$ & Latejante & Leve a grave & $\begin{array}{l}\text { Minutos a } \\
\text { horas }\end{array}$ & $\begin{array}{l}\text { Mecânico, } \\
\text { comida, frio, calor }\end{array}$ & - \\
\hline Tumores orofaciais & Variável & $\begin{array}{l}\text { Variável } \\
\text { (atípico) }\end{array}$ & Grave & $\begin{array}{l}\text { Leve a } \\
\text { moderado }\end{array}$ & $\begin{array}{l}\text { Movimento da } \\
\text { mandíbula }\end{array}$ & $\begin{array}{l}\text { Frequentemente } \\
\text { sinais neurológicos }\end{array}$ \\
\hline
\end{tabular}

FONTE: Agostoni et al., 2005. p. S73. Adaptado. 
A fisiopatologia da DFA é ainda desconhecida e controversa. Embora não existam sintomas neurológicos típicos (hipoestesia, alodínia, hiperestesia), podem haver anormalidades sensitivas discretas (Woda, 2007). Foram observadas alterações sensitivas térmicas ao frio $(47,8 \%)$, mecânicas $(46,5 \%)$ e dolorosas $(67,4 \%)$ na DFA (List, Leijon et al., 2008; Baad-Hansen, Pigg et al., 2013). A sensibilidade intraoral no lado da dor apresenta diferenças significativas em $78,3 \%$ dos pacientes, sendo discretas em 94\% na comparação com o lado oposto em relação ao limiar de dor à pressão, somação temporal, alodínia mecânica dinâmica e limiar de dor ao quente.

Há anormalidade reflexa de $\mathrm{R} 3$ em $70,6 \%$ dos doentes com DFA caracterizada por diminuição de amplitude no lado da dor (Jääskeläinen, Forssell et al., 1999). Porém, não foi encontrada correlação clínica. A investigação de receptores de dopamina D2 detectou aumento de captação no putamen e diminuição na razão D1/D2, o que sugere alteração dopaminérgica nigroestriatal na DFA. Esses achados são semelhantes àqueles encontrados em doentes com síndrome da ardência bucal, indicando o sistema dopaminérgico nigroestriatal em associação à dor crônica orofacial (Hagelberg, Forssell et al., 2003).

\subsubsection{Tratamento da DFA}

O tratamento da DFA pode ser farmacológico com medicamentos tópicos, e através de procedimentos invasivos ou não invasivos (EMT). 


\subsubsection{Tratamento farmacológico da DFA}

O tratamento farmacológico das síndromes dolorosas, incluindo os diferentes tipos de dores crônicas é, de modo geral, baseado nos mecanismos fisiopatológicos. Ele inclui antidepressivos (tricíclicos, inibidores seletivos de receptação de serotonina, inibidores duais), anticonvulsivantes, analgésicos (opioides e não opioides), medicações tópicas (lidocaína, capsaicina), e outras, como canabinóides, mexilentina, agonista de NMDA e toxina botulínica A (Hansson, Attal et al.; 2009; Oliveira, 2009; Attal, Cruccu et al.; 2010; Stump, 2012).

Os antidepressivos tricíclicos isoladamente ou em associação são as drogas de escolha para o tratamento da DFA (Melis, Lobo et al., 2003), sendo a amitriptilina a primeira opção. São utilizadas doses baixas (25 a 100 mg), administradas uma única vez ao dia, e que promovem melhora inicial de $30 \%$ a $40 \%$ (Frediani, 2005). Vários autores observaram cerca de $69 \%$ de alívio da DFA com antidepressivos tricíclicos (Marbach, 1978; Brooke, 1980; Kreisberg, 1982; Schnurr, Brooke, 1992; Frediani, 2005), porém há controvérsias (Reik Jr., 1984). Essa melhora é obtida somente após três a quatro semanas de uso.

O mecanismo de ação dos antidepressivos tricíclicos na dor ainda não está completamente elucidado, e envolve mudanças no metabolismo de neurotransmissores e monoaminas (particularmente noraepinefrina - NE e serotonina - 5-HT) no corno posterior da medula espinhal. A inibição da recaptação da serotonina reduz a aferência sensitiva. Apresenta longa meia- 
vida (8 a 89 horas), o que facilita o uso, porém seus efeitos colaterais dificultam a adesão (Ward e Azzaro, 2005; Brunton, Parker et al., 2008; Galhardoni, Bonfiglioli et al., 2012). Entre esses efeitos, podemos destacar boca seca, tontura, constipação, visão borrada, palpitações, taquicardia, diminuição do apetite, náusea e/ou vômito, fraqueza, confusão, desorientação e ganho de peso. Esses efeitos tendem a passar com o tempo de uso (Ward, Azzaro, 2005; Galhardoni, Bonfiglioli et al., 2012). Outras classes de medicamentos, como anticonvulsivantes e analgésicos (opioides e não opioides), apresentam pouca ou nenhuma resposta nos quadros de DFA (Frediani, 2005).

\subsubsection{Medicamentos tópicos na DFA}

Tratamentos tópicos incluem: anestésicos (benzocaína 15\%, ametocaína 1,7\%, EMLA 6\%), dexametasona e capsaicina (0,025\%) (Trace, Barbieri et al., 2013). Eles promovem um significante, porém incompleto, alívio da dor (Rees e Harris, 1978; Bates Jr., Stewart, 1991; List, Leijon et al., 2006), principalmente da alodínia (Trace, Barbieri et al., 2013). Sua eficácia ainda é controversa (Graff-Radford, Solberg, 1992), havendo a necessidade de mais estudos sobre o tema (Trace, Barbieri et al., 2013). 


\subsubsection{Procedimentos Cirúrgicos na DFA}

Os procedimentos neurocirúrgicos utilizados para tratar a DFA são poucos. A radiofrequência do gânglio esfenopalatino apresenta nível de evidência de $2 \mathrm{C}+$, conforme descrito pelos guidelines de medicina baseada em evidência - eficácia apenas em estudos observacionais; análise de risco/benefício -, sendo que, por nível de evidência, entende-se a hierarquização da pesquisa e a abordagem metodológica adotada para o desenvolvimento do estudo (Cornelissen, van Kleef et al., 2009). Em um artigo, houve $21 \%$ de melhora completa da DFA e $65 \%$ de melhora boa ou moderada (Bayer, Racz et al., 2005). Nobrega, et al. (Nóbrega, Teixeira et al., 2000) utilizaram a nucleotratotomia trigeminal estereotáxica para tratar dois doentes com DFA, com alívio total da dor.

Doentes com DFA e neuralgia idiopática do trigêmeo associada foram tratados com anestésico local e esteroides na fossa pterigopalatina, sendo que $80 \%$ deles tiveram analgesia completa com 15 minutos de injeção, independente do ramo afetado (Nader, Kendall et al., 2013). Bloqueio do

gânglio estrelado pode aliviar os sintomas pela associação do sistema neurovegetativo na dor (Shanthanna, 2013). Outros procedimentos controversos incluem a descompressão trigeminal vascular e implante de estimuladores cerebrais profundos. Porém, há a necessidade de mais estudos (Cornelissen, van Kleef et al., 2009). De modo geral, a cirurgia traz alívio temporário, é um procedimento invasivo e apresenta riscos que precisam ser considerados, como infecções, hemorragias, sequelas 
neurológicas, morbidades e mortalidade.

\subsubsection{Estimulação Magnética Transcraniana (EMT)}

Devido à melhora parcial da dor da DFA através de fármacos e procedimentos cirúrgicos, a EMT tem se apresentado como uma alternativa promissora e não invasiva que permite a modulação elétrica cerebral.

A história do uso da eletricidade para o tratamento do sintoma álgico é antiga. No período clássico, Aristóteles, Galeno e Plutarco já utilizavam o peixe elétrico para tratar a dor. No Império Romano, Largus, médico do imperador Claudius (43-48 d.C.), travava a cefaleia utilizando o peixetorpedo. No final do século XVIII, é criada a primeira pilha voltaica por Alessandro Volta, e Luigi Galvani realiza experimentos em sapos demonstrando a contração muscular. No século XIX, inicia-se a estimulação elétrica de cadáveres por Giovanni Aldini, sobrinho de Galvani e também físico (Brunoni, Boggio et al., 2012). Finalmente, Roberts Bartholow realiza a primeira estimulação do córtex cerebral de uma pessoa desperta, simultaneamente ao mapeamento cortical de Penfield, protocolo que ficou conhecido como procedimento de Montreal (Brunoni, Boggio et al., 2012).

Foi somente em 1985 que Anthony Baker apresentou uma máquina de EMT capaz de gerar potenciais evocados motores sem causar dor. A EMT utiliza-se da Lei de Faraday (1831), onde a passagem da corrente elétrica pela bobina gera um campo eletromagnético que é capaz de promover diferença de potencial a alguns centímetros de distância da bobina e, quando aplicado a tecido vivo, leva à despolarização de neurônios através 
da criação de corrente elétrica induzida (Huerta e Volpe, 2009). Na EMT, a corrente elétrica percorre uma bobina de $8 \mathrm{~cm}$ de diâmetro em um milissegundo. Um campo eletromagnético de 1,9 Tesla gera corrente induzida no córtex cerebral a aproximadamente $2 \mathrm{~cm}$ de distância da bobina, levando à despolarização de neurônios (Edwards, Talelli et al., 2008; Huerta, Volpe, 2009; Lefaucheur , 2009).

O uso da neuromodulação para o tratamento da dor crônica de origem central iniciou-se em 1991 com Tsubokawa et al. (1991). O giro pré-central (córtex sensitivo primário) era estimulado para aliviar a dor neuropática de origem central após acidente vascular encefálico, sendo que a hipótese aceita era a de haver modulação rostrocaudal de neurônios terciários sensibilizados. Esse estudo demonstrou em gatos uma atenuação da hiperatividade anormal do tálamo após transecção espinotalâmica. Com essas descobertas foi possível iniciar a exploração do córtex motor primário para a estimulação tanto invasiva quanto não invasiva (Lefaucheur, Hatem et al., 2006; Edwards, Talelli et al., 2008; Ciampi de Andrade, 2013).

Em modelos animais de dor crônica, a estimulação elétrica do cortéx motor (EECM) inibe a hiperatividade de neurônios talâmicos desaferentados, sendo que neurônios do corno dorsal da coluna vertebral apresentam uma diminuição de resposta a estímulos mecânicos. Assim, há um aumento do limiar nociceptivo mecânico (Fonoff, Dale et al., 2009). O implante subdural do córtex motor inibe o comportamento doloroso crônico causado pela constrição crônica do nervo ciático em ratos na pata contralateral, mas não na pata ipsilateral (Pagano, Assis et al., 2011). 
Pagano et al. demonstraram que a estimulação epidural do córtex motor de ratos reverte a hiperalgesia e alodínia mecânica devido à inibição da medula espinal e de núcleos talâmicos, e consequente ativação da substância cinzenta periaquedutal, do córtex cingulado anterior e de núcleos da amígdala (Pagano, Fonoff et al., 2012).

Um estudo realizado por Fonoff et al. (2011) em humanos que apresentavam síndrome complexa de dor regional, observou-se que a estimulação do córtex motor influencia os níveis de opioides endógenos no giro anterior do cíngulo e na substância cinzenta periaquedutal, e que o EEMC pode modular as informações aferentes ascendentes da medula espinal na dor neuropática de forma recíproca tanto retrógrada quando anterógrada nas projeções corticotalâmicas.

Por outro lado, a estimulação não invasiva é uma boa alternativa para o tratamento da dor crônica. Atualmente, a EMT pode ser usada de duas formas (Cruccu, Aziz et al., 2007; Chen, Cros et al., 2008; Rossi, Hallett et al., 2009): como ferramenta neurofisiológica para a realização de potenciais evocados motores e mensuração de padrões corticais (por exemplo, excitabilidade cortical) (Lefaucheur, 2006; Lefaucheur, Drouot et al., 2006; Chen, Cros et al., 2008; Mhalla, de Andrade et al., 2010), e como tratamento de doenças psiquiátricas e neurológicas, como a dor neuropática (Lefaucheur, 2004; Lefaucheur, 2006; Lefaucheur, Drouot et al., 2006), a fibromialgia (Mhalla, de Andrade et al., 2010; Mhalla, Baudic et al., 2011) e a síndrome complexa de dor regional (Picarelli, Teixeira et al., 2010). 
O campo magnético é produzido a partir do posicionamento da bobina próxima ao couro cabeludo, que o atravessa por uma corrente elétrica breve e de grande intensidade, gerando linhas de campo perpendiculares ao plano da bobina (Figura 1) (Edwards, Talelli et al., 2008).

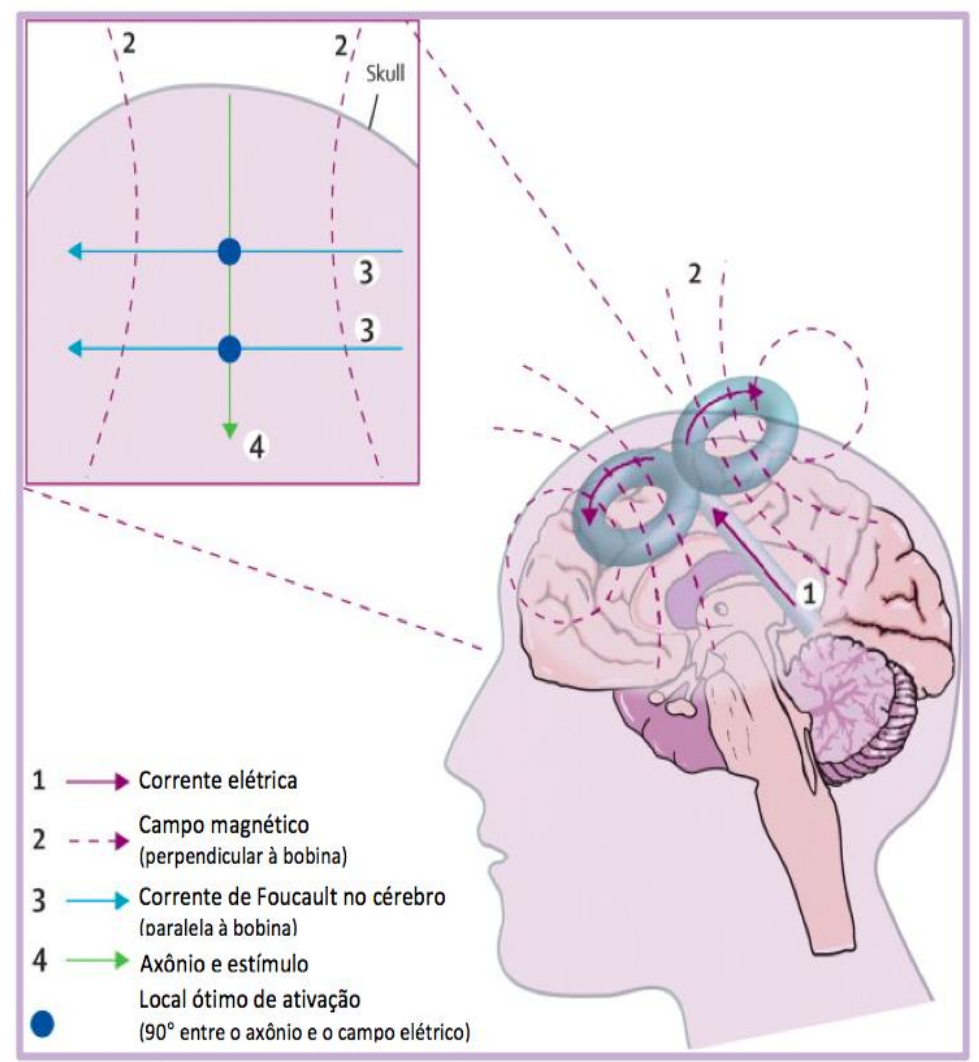

Figura 1 - Mecanismo de ação da EMT FONTE: Edwards et al., 2008.

A corrente induzida no córtex cerebral é perpendicular a essas linhas de campo. Dessa forma, em um meio homogêneo, a corrente produzida no cérebro é paralela à orientação da bobina e se distribui formando um gradiente, sendo maior nas proximidades da bobina e menor à medida que se afasta dela. A corrente é mais fraca nas proximidades do centro da bobina e, no centro propriamente dito, não há corrente (Huerta e Volpe, 
2009; Araújo, Iglesio et al., 2011). As únicas regiões do cérebro que provocam um efeito mensurável ao serem estimuladas são o córtex motor primário, que provoca contrações nos grupos musculares correspondentes à região estimulada que podem ser, inclusive, medidas em um aparelho de eletromiografia (EMG), e o córtex visual (Davey, Smith et al., 1999; Groppa, Oliviero et al., 2012).

O córtex cingulado anterior e a substância cinzenta periaquedutal são ativados de forma direta provavelmente porque essas estruturas contêm elevada densidade de receptores opioides pertencentes à rede subcortical que é ativada durante a analgesia opioide em humanos. De fato, foi demonstrada a ativação endógena do sistema opioide tanto em modelos animais como em estudos com doentes com dor crônica que utilizavam eletrodo epidural (Fonoff, Dale et al., 2009; Fonoff, Hamani et al., 2011; Pagano, Fonoff et al., 2012). Experimentalmente, demonstrou-se que a ativação dos receptores $\mu$-opioides aumenta a liberação de dopamina na região tegmental ventral, modulando a transmissão gabaérgica (de Andrade, Mhalla et al., 2011).

O EMTr ativa preferencialmente os axônios dos corpos celulares dos neurônios corticais. A EMTr de alta frequência $(\geq 5 \mathrm{~Hz})$ promove facilitação, sendo que a de baixa frequência $(\leq 1 \mathrm{~Hz})$ promove inibição. Existem outros parâmetros que também modulam os efeitos da EMTr, como: a orientação da bobina, a corrente elétrica induzida e a direção da corrente no encéfalo (Edwards, Talelli et al., 2008; Lefaucheur, 2009; Galhardoni, Teixeira et al., 2010; Ciampi de Andrade, 2013). 
A EMTr tende a reduzir a intensidade da dor entre 30\% e 40\%(Ciampi de Andrade, 2013). Uma única estimulação do córtex motor primário (M1) pode aliviar o sintoma álgico (Khedr, Kotb et al., 2005), e cinco sessões consecutivas em pacientes com dor crônica a $20 \mathrm{~Hz}$ promovem melhora significativa que dura até duas semanas após o tratamento. Contudo, ainda há controvérsias em relação ao uso da baixa frequência para inibição de vias neurais (Ciampi de Andrade, 2013). Os resultados apresentam-se similares ao placebo (Defrin, Grunhaus et al. 2007).

Não existe na literatura relatos de estudos de EMTr para avaliação e tratamento de DFA, e há poucos estudos que utilizaram a EMT na dor facial. Esses estudos podem ser observados na Tabela 3. 
Tabela 3 - Estudos sobre EMTr em dor crônica incluindo dor facial

\begin{tabular}{|c|c|c|c|c|c|c|c|}
\hline Autor & $\begin{array}{c}\text { Síndrome } \\
\text { dolorosa e } \\
\text { número de } \\
\text { participantes }\end{array}$ & $\begin{array}{l}\text { Tipo de } \\
\text { estudo }\end{array}$ & $\begin{array}{l}\text { Orientação } \\
\text { da corrente } \\
\text { induzida }\end{array}$ & $\begin{array}{l}\text { Tipo de } \\
\text { bobina, } \\
\text { alvo, } \\
\text { frequência, } \\
\text { intensidade } \\
\text { e número } \\
\text { total de } \\
\text { pulsos }\end{array}$ & $\begin{array}{l}\text { Número } \\
\text { de } \\
\text { sessões }\end{array}$ & $\begin{array}{l}\text { Período de } \\
\text { avaliação }\end{array}$ & Desfecho \\
\hline Reid et al., 2001 & $\begin{array}{l}\text { Dor facial } \\
\text { bilateral }(=1)\end{array}$ & $\begin{array}{l}\text { Relato de } \\
\text { caso }\end{array}$ & n.d. ${ }^{*}$ & $\begin{array}{c}\text { Tipo de } \\
\text { bobina } \\
\text { (n.d.), } \\
20 \mathrm{~Hz}, \\
100 \% \text { do } \\
\text { LMR, no de } \\
\text { pulsos (n.d) }\end{array}$ & 14 & $\begin{array}{c}\text { Entrada e } \\
\text { saída, EVA*; } \\
\text { Escala de } \\
\text { Avaliação de } \\
\text { depressão de } \\
\text { Asberg; } \\
\text { Inventário de } \\
\text { depressão de } \\
\text { Beck }\end{array}$ & $\begin{array}{l}\text { Diminuição da dor em } \\
42 \%\end{array}$ \\
\hline $\begin{array}{l}\text { Lefaucheur et } \\
\text { al., } 2001\end{array}$ & $\begin{array}{r}\text { DNe central } \\
\text { (talâmica }=7 \\
\text { trigeminal }=7 \text { ) }\end{array}$ & $\begin{array}{l}\text { Duplo-cego, } \\
\text { cruzado, } \\
\text { controlado por } \\
\text { placebo }\end{array}$ & $\begin{array}{l}\text { Póstero- } \\
\text { anterior }\end{array}$ & $\begin{array}{c}\mathrm{F} 8, \mathrm{M} 1 \mathrm{da} \\
\text { mão, } 10 \mathrm{~Hz}, \\
80 \% \text { do } \\
\text { LMR, } \\
1000 \mathrm{p} .\end{array}$ & 1 & $\begin{array}{l}12 \text { dias após } \\
\text { a estimulação } \\
\text { EVA }^{*}\end{array}$ & $\begin{array}{c}\text { Efeito analgésico principal } \\
\text { encontrado entre o } 2^{\circ} \text { e } 0 \\
4^{\circ} \text { dia de estimulação }\end{array}$ \\
\hline
\end{tabular}

Continua na próxima página 
Continuação da página anterior

Tabela 3 - Estudos sobre EMTr em dor crônica envolvendo dor facial

\begin{tabular}{|c|c|c|c|c|c|c|c|}
\hline Autor & $\begin{array}{l}\text { Síndrome } \\
\text { dolorosa e } \\
\text { número de } \\
\text { participantes }\end{array}$ & $\begin{array}{l}\text { Tipo de } \\
\text { estudo }\end{array}$ & $\begin{array}{l}\text { Orientação } \\
\text { da corrente } \\
\text { induzida }\end{array}$ & $\begin{array}{c}\text { Tipo de } \\
\text { bobina, alvo, } \\
\text { frequência, } \\
\text { intensidade e } \\
\text { número total } \\
\text { de pulsos }\end{array}$ & $\begin{array}{l}\text { Número } \\
\text { de } \\
\text { sessões }\end{array}$ & $\begin{array}{l}\text { Período de } \\
\text { avaliação }\end{array}$ & Desfecho \\
\hline $\begin{array}{l}\text { Lefaucheur et } \\
\text { al., } 2004\end{array}$ & $\begin{array}{c}\text { DNe* central } \\
\text { (dor pós ave } \\
=36 \text { ); periférica } \\
\text { (trigeminal =12; } \\
\text { plexo braquial } \\
=12 \text { ) }\end{array}$ & $\begin{array}{l}\text { Duplo-cego, } \\
\text { cruzado, } \\
\text { controlado por } \\
\text { placebo }\end{array}$ & $\begin{array}{l}\text { Póstero- } \\
\text { anterior }\end{array}$ & $\begin{array}{l}\text { F8, M1 da } \\
\text { mão, } 10 \mathrm{~Hz} \text {, } \\
80 \% \text { do LMR, } \\
\text { 1000p. }\end{array}$ & 1 & $\begin{array}{c}\text { Logo após a } \\
\text { sessão }\end{array}$ & $\begin{array}{l}\text { Melhora da lesão do nervo } \\
\text { trigêmeo, melhora da dor } \\
\text { localizada na face e } \\
\text { ausência de perda sensitiva }\end{array}$ \\
\hline $\begin{array}{l}\text { Khedr et al., } \\
2005\end{array}$ & $\begin{array}{l}\text { DNe central (dor } \\
\text { pós-ave }=24 \text { ) } \\
\text { (trigeminal }=24 \text { ) }\end{array}$ & $\begin{array}{l}\text { Duplo-cego, } \\
\text { cruzado, } \\
\text { controlado por } \\
\text { placebo }\end{array}$ & $\begin{array}{l}\text { Latero-medial } \\
\text { (a 45 da } \\
\text { linha média } \\
\text { sagital) }\end{array}$ & $\begin{array}{l}\text { F8, M1 da } \\
\text { mão, } 20 \mathrm{~Hz} \text {, } \\
80 \% \text { do LMR, } \\
2000 \mathrm{p} .\end{array}$ & 5 & $\begin{array}{l}\text { 21dias/efeito } \\
\text { duradouro } 14 \\
\text { dias após o final } \\
\text { da EMT }\end{array}$ & $\begin{array}{l}\text { Redução da EVA em } 45 \% \\
\text { do basal após a } 5^{a} \text { sessão } \\
\text { e } 40 \% \text { após duas semanas }\end{array}$ \\
\hline $\begin{array}{l}\text { Hirayama et al., } \\
2006\end{array}$ & $\begin{array}{l}\text { DNe central (dor } \\
\text { pós-ave } \\
=14) ; \text { (trigeminal } \\
=3 \text { ) e DNe } \\
\text { periférica }(=3)\end{array}$ & $\begin{array}{l}\text { Duplo-cego, } \\
\text { cruzado, } \\
\text { controlado por } \\
\text { placebo }\end{array}$ & n.d. & $\begin{array}{l}\text { F8, M1, S1, } \\
\text { CPM, SMA da } \\
\text { área } \\
\text { dolorosa, } \\
\text { 5Hz, 90\% do } \\
\text { LMR, 500p. }\end{array}$ & 1 & $\begin{array}{l}\text { 3horas após } \\
\text { com EVA, } \\
\text { Questionário de } \\
\text { dor McGill }\end{array}$ & $\begin{array}{c}\text { Efeito duradouro de } 3 \text { horas } \\
\text { após EMT }\end{array}$ \\
\hline
\end{tabular}

Continua na próxima página 
Continuação da página anterior

Tabela 3 - Estudos sobre EMTr em dor crônica envolvendo dor facial

\begin{tabular}{|c|c|c|c|c|c|c|c|}
\hline Autor & $\begin{array}{l}\text { Síndrome } \\
\text { dolorosa e } \\
\text { número de } \\
\text { participantes }\end{array}$ & $\begin{array}{l}\text { Tipo de } \\
\text { estudo }\end{array}$ & $\begin{array}{l}\text { Orientação } \\
\text { da corrente } \\
\text { induzida }\end{array}$ & $\begin{array}{l}\text { Tipo de } \\
\text { bobina, } \\
\text { alvo, } \\
\text { frequência, } \\
\text { intensidade } \\
\text { e número } \\
\text { total de } \\
\text { pulsos } \\
\end{array}$ & $\begin{array}{c}\text { Número } \\
\text { de } \\
\text { sessões }\end{array}$ & $\begin{array}{l}\text { Período de } \\
\text { avaliação }\end{array}$ & Desfecho \\
\hline $\begin{array}{l}\text { Lefaucheur et } \\
\text { al., } 2006\end{array}$ & $\begin{array}{c}\text { DNe facial } \\
(=18), \text { mão } \\
(=18) \text { [origem } \\
\text { central e } \\
\text { periférica] }\end{array}$ & $\begin{array}{l}\text { Duplo-cego, } \\
\text { cruzado, }\end{array}$ & $\begin{array}{l}\text { Póstero- } \\
\text { anterior }\end{array}$ & $\begin{array}{c}\text { F8, M1 da } \\
\text { face, braço, } \\
\text { mão, } 10 \mathrm{~Hz}, \\
90 \% \text { do } \\
\text { LMR, } \\
2000 \mathrm{p} .\end{array}$ & 1 & $\begin{array}{l}1 \text { semana } \\
\text { antes e após } \\
\text { a sessão de } \\
\text { EMT }\end{array}$ & $\begin{array}{l}\text { Melhora da dor da mão } \\
\text { com a EMT na área da } \\
\text { "face" e melhora da dor } \\
\text { facial com a EMT na área } \\
\text { da "mão" }\end{array}$ \\
\hline $\begin{array}{l}\text { Siqueira et al., } \\
2010\end{array}$ & DFA $(n=1)$ & $\begin{array}{l}\text { Relato de } \\
\text { caso }\end{array}$ & $\begin{array}{l}\text { Perpendi- } \\
\text { cular ao } \\
\text { córtex }\end{array}$ & $\begin{array}{c}\text { F8, CPFDL } \\
\text { esquerdo, } \\
10 \mathrm{~Hz}, \\
120 \% \text { do } \\
\text { LMR, no de } \\
\text { pulsos } \\
\text { (n.d.) }\end{array}$ & 1 & $\begin{array}{l}3 \text { e } 6 \text { dias e } 2 \\
\text { meses após a } \\
\text { sessão EMT } \\
\text { com EVA }\end{array}$ & $\begin{array}{l}\text { Redução dor avaliada } \\
\text { através da EVA para } 3\end{array}$ \\
\hline
\end{tabular}

Legenda: $n d$ = não descrito; $M 1=$ córtex motor primário; $E V A=$ escala visual analógica; $D N e=$ dor neuropática, $F 8=$ bobina figura de oito; $L M R=$ limiar motor de repouso; $\mathrm{S} 1$ = córtex sensitivo primário, $\mathrm{CPM}=$ córtex pré-motor; $\mathrm{SMA}=$ área suplementar motora; EMT= estimulação magnética transcraniana; CPFDL= córtex pré-frontal dorsolateral. 
Paralelamente ao desenvolvimento da EMTr como ferramenta terapêutica, a utilização em neurofisiologia para exploração das vias corticais e do córtex levou à observação de alterações no recrutamento e facilitação de moto-neurônios corticais em pacientes com lesão da medula parcial e incompleta (Davey, Smith et al., 1999). Foi observado que doentes com fibromialgia apresentavam alterações corticais de receptores do ácido gama amino-butírico (GABA) A e B (Salerno, Thomas et al., 2000).

Lefaucheur (2006) observou que doentes com dor neuropática apresentavam anormalidades na inibição intracortical que poderiam ser restauradas após o tratamento com EMTr em alta frequência. Assim, a excitabilidade cortical passou a ser utilizada na investigação do funcionamento do córtex motor, incluindo vias interneuronais glutamatérgicas, gabaérgicas e canais iônicos.

Atualmente, existem 25 diferentes medidas que compõem o conjunto denominado de excitabilidade cortical (Tabela 4) (Ciampi de Andrade, 2013). 
Tabela 4 - Revisão dos principais parâmetros de excitabilidade cortical

\begin{tabular}{|c|c|c|c|}
\hline Parâmetro & Descrição & Principal valor clínico & Autor \\
\hline Curva de Recrutamento & $\begin{array}{l}\text { Também chamada de curva estímulo-resposta ou } \\
\text { input-output. Refere-se ao aumento da amplitude } \\
\text { do PEM com aumentos da intensidade de } \\
\text { estimulação pela EMT. }\end{array}$ & $\begin{array}{l}\text { Ativa neurônios menos excitáveis ou espacialmente } \\
\text { distanciados do centro de ativação pela EMT. São } \\
\text { relacionadas à força das projeções corticoespinais e é } \\
\text { mais inclinada em músculos que possuem LM mais } \\
\text { baixos. Sua inclinação é aumentada por agentes } \\
\text { adrenérgicos e diminúda por bloqueadores de canais } \\
\text { iônicos e por agonistas Gabaérgicos }\end{array}$ & et al., \\
\hline $\begin{array}{l}\text { Tempo de Condução } \\
\text { Motora central/ Técnica } \\
\text { de Tripla Estimulação }\end{array}$ & $\begin{array}{l}\text { Estimativa do tempo de condução de fibras } \\
\text { corticoespinais entre córtex motor, neurônios } \\
\text { motores espinais ou bulbares. }\end{array}$ & $\begin{array}{l}\text { Uma das técnicas utilizadas para sua obtenção - } \\
\text { técnica de estimulação tripla - permite a quantificação } \\
\text { de falhas de condução central causadas por } \\
\text { excitabilidade reduzida ou perda de neurônios motores } \\
\text { corticais por bloqueio de condução ou perda de axônios } \\
\text { corticoespinais. Há estudos utilizando esse parâmetro } \\
\text { em mielopatias compressivas e esclerose lateral } \\
\text { amiotrófica. }\end{array}$ & $\begin{array}{l}\text { Magistris et } \\
\text { al.,1999; Roth e } \\
\text { Magistris, } 1989\end{array}$ \\
\hline
\end{tabular}


Tabela 4 - Revisão dos principais parâmetros de excitabilidade cortical

Continuação da página anterior

\begin{tabular}{|c|c|c|c|}
\hline Parâmetro & Descrição & Principal valor clínico & Autor \\
\hline $\begin{array}{l}\text { Inibição } \\
\text { Intracortical de } \\
\text { Intervalo Curto } \\
\text { (IICIC) }\end{array}$ & $\begin{array}{l}\text { Quando um estímulo condicionante } \\
\text { (EC) subliminar (abaixo do LM) é } \\
\text { administrado de } 1 \text { a } 6 \text { milissegundos } \\
\text { precedendo um estímulo teste (ET) } \\
\text { supraliminar (acima do LM) há uma } \\
\text { redução do PEM, chamada de IICIC. }\end{array}$ & $\begin{array}{l}\text { A IICIC um fenômeno complexo dependente de sistema interneuronal } \\
\text { localizado no córtex motor, de limiares baixos que representam a interação } \\
\text { entre um sistema inibitório potente e um facilitatório mais fraco. } \\
\text { Aumenta com aumentos do ET e do EC (padrão de curva em U). } \\
\text { Provavelmente tem ação de filtrar programas motores indesejados e facilitar } \\
\text { os que têm propósito funcional. É aumentado por agonistas GABAA, } \\
\text { antiglutamatérgicos e dopaminérgicos, não sendo influenciado por } \\
\text { bloqueadores de canais iônicos. } \\
\text { Reduzido em doentes com fibromialgia e dor neuropática }\end{array}$ & $\begin{array}{l}\text { Kujirai et al., 1993; } \\
\text { Mhalla et al., } 2010\end{array}$ \\
\hline $\begin{array}{l}\text { Inibição } \\
\text { Intracortical de } \\
\text { Intervalo Longo } \\
\text { (IICIL) }\end{array}$ & $\begin{array}{l}\text { EC supralimiar administrado } 50-200 \mathrm{~ms} \\
\text { antes de ET, reduzem os PEM dos ET. }\end{array}$ & $\begin{array}{l}\text { É um fenômeno predominantemente cortical. Depende de neurônios } \\
\text { corticoespinais de limiares mais baixos e não são influenciados por } \\
\text { contrações motoras voluntárias. É mediado por receptores GABAB. }\end{array}$ & $\begin{array}{l}\text { Valls-Sole et al., } \\
1992\end{array}$ \\
\hline $\begin{array}{l}\text { Facilitação } \\
\text { Intracortical de } \\
\text { Intervalo Curto }\end{array}$ & $\begin{array}{l}\text { EC e ET próximos do LM ou EC supra } \\
\text { e ET infralimiar, em ambos os casos } \\
\text { separados por } 1-4,5 \mathrm{~ms} \text { fazem com } \\
\text { que o ET seja facilitado }\end{array}$ & $\begin{array}{l}\text { Estímulos com EC despolarizam parcialmente os axônios de neurônios } \\
\text { responsáveis pela geração de ondas i, tornando-os hiperexcitáveis. O } \\
\text { segundo pulso (ET) acaba de despolarizá-los. Depende de receptores } \\
\text { GABAA. }\end{array}$ & Tokimura et al., 1996 \\
\hline $\begin{array}{l}\text { Facilitação } \\
\text { Intracortical }\end{array}$ & $\begin{array}{l}\text { EC infra e ET supralimiares causam } \\
\text { aumento dos PEM dos ET. }\end{array}$ & Dependente de receptores glutamatérgicos (NMDA) & Ziemann et al., 1996 \\
\hline
\end{tabular}

FONTE: Ciampi de Andrade, 2013.

Legenda: EC: estímulo condicionante, EMT: estimulação magnética transcraniana, ET: estímulo teste, GABA: ácido gama-aminobutírico, ms: milissegundo, NMDA: n-metil-d-aspartato, PEM: potencial evocado motor. 


\section{CASUÍSTICA E MÉTODOS}




\subsection{Casuística}

Neste estudo duplamente encoberto aleatorizado, foram avaliados 29 doentes do Ambulatório de Algias craniofaciais da Divisão de Neurologia do Hospital das Clínicas da Faculdade de Medicina da Universidade de São Paulo (HC-FMUSP), diagnosticados com DFA segundo os critérios da Classificação Internacional de Cefaleia da International Headache Society (IHS) (IHS 2004). A amostra foi calculada através do software G*Power 3.1 (Faul, Erdfelder et al. 2007) (Figura 2). Para a avaliação comparativa da excitabilidade cortical, realizou-se um corte transversal para compor uma amostra de 28 indivíduos saudáveis pareados quanto a idades e gênero (grupo controle). Os doentes eram dezesseis mulheres (55,2\%), com média de idade de $55,79 \pm 12,15$ anos. O grupo de controle foi composto por dezesseis mulheres $(57,1 \%)$, com média de idade de $50,68 \pm 12,31$ anos (respectivamente: $p=0,120$, exato de Fisher; $p=0,547$, t de Student).

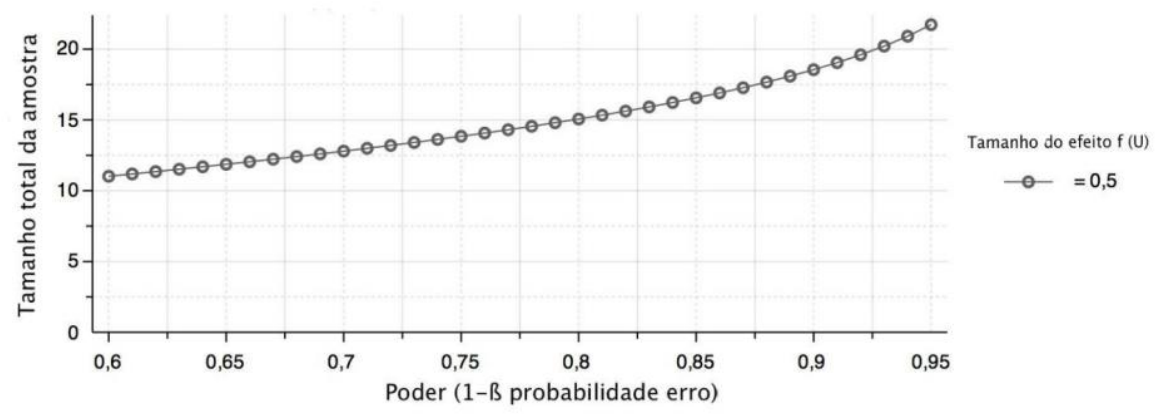

Figura 2 - Cálculo do tamanho da amostra segundo o programa G*Power 3.1 Teste Anova: Medidas repetidas, interação entre sujeitos. Número de grupos=2; Número de medições $=5$; Correção de não esfericidade $\varepsilon=1 ; \alpha$ erro de probabilidade $=0,05$. Tamanho do Efeito $f(U)=0,5$. 
Os doentes foram distribuídos de forma aleatória através de um programa open source, disponível em www.randomizer.org, de forma a compor dois subgrupos: o primeiro, com quatorze doentes, submetido ao tratamento com EMTr sham (Grupo Placebo), e o segundo, com quinze doentes, submetido ao tratamento com EMTr ativo (Grupo Ativo). A distribuição dos doentes está apresentada segundo o diagrama Consort (Turner, Shamseer et al. 2012) na Figura 3.

Este estudo foi aprovado pela Comissão de Ética do Hospital das Clínicas (ANEXO 1) e registrado na plataforma Clinical Trials.gov sob o número NCT01746355 (ANEXO 2). Somente participaram deste estudo doentes e voluntários que assinaram o termo de consentimento livre e esclarecido (TCLE) (ANEXO 3). 


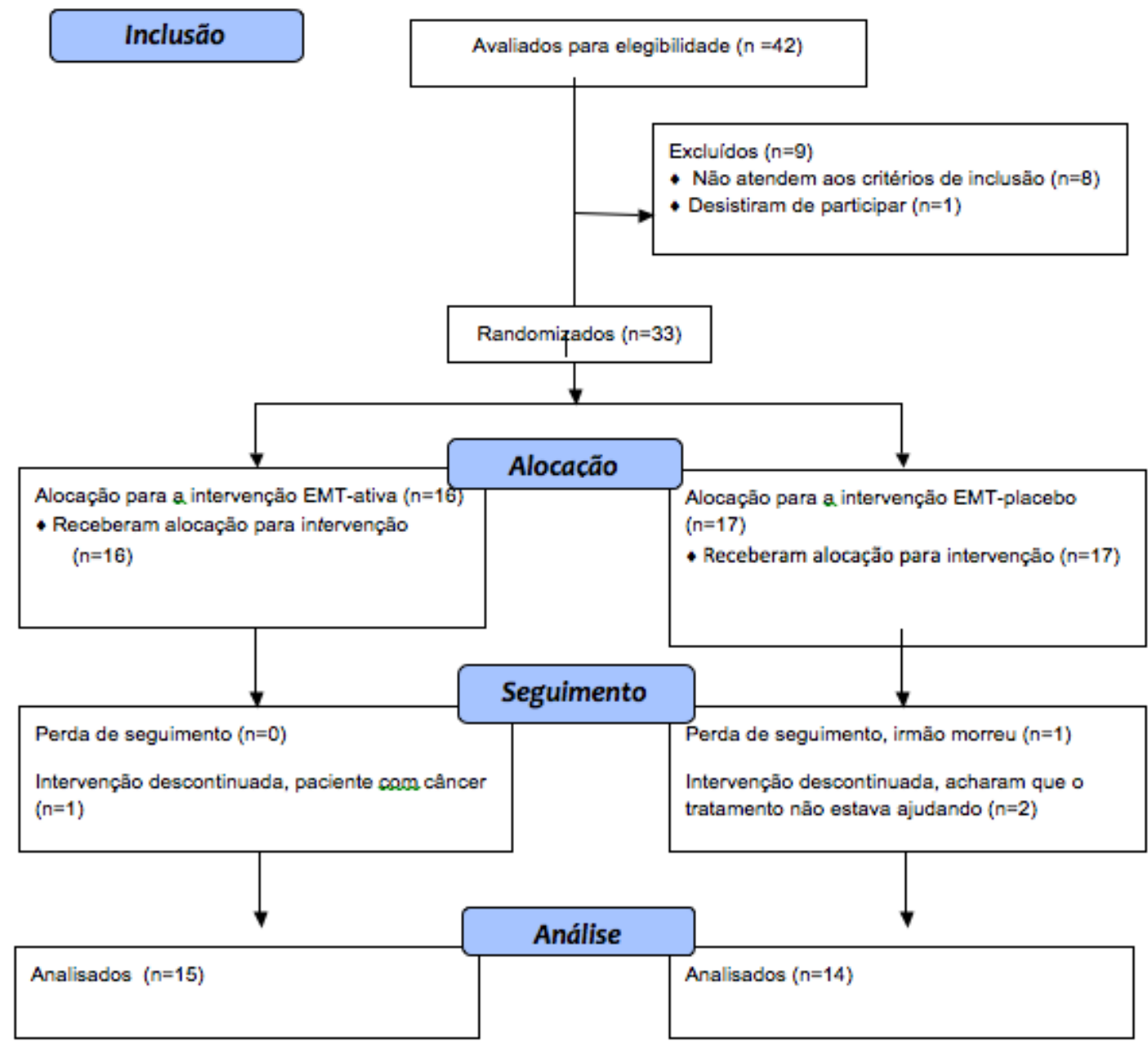

Figura 3 - Diagrama Consort do estudo 


\subsubsection{Critérios de Inclusão (grupos de estudo) (ANEXO 4)}

$>\quad$ Idade superior a 18 anos;

$>$ Apresentar-se em tratamento continuado para a DFA no ambulatório de Algias Cranio-Faciais da Divisão de Neurologia do HCFMUSP durante os últimos três meses, sem interrupções;

> Atender ao critério de resistência farmacológica documentada a tratamento clínico, definida em nosso estudo como persistência de dor após um mínimo de dois tratamentos farmacológicos adequados, incluindo pelo menos um antidepressivo/anticonvulsivante e/ou gabapentinoide. A adequação dos tratamentos foi definida pelo uso de fármacos em doses consideradas adequadas na literatura ou abaixo do limiar de efeitos colaterais (Hansson, Attal et al., 2009);

Apresentar pontuação na escala visual analógica (EVA) maior que seis por mais de três meses consecutivos antes de iniciar o tratamento.

\subsubsection{Critérios de Inclusão (grupos controle)}

$>\quad$ Idade superior a 18 anos;

$>\quad$ Ausência de quaisquer queixas de dor orofacial ou em outra localização corpórea. 


\subsubsection{Critérios de Exclusão (grupo de estudo e controles)}

$>$ Encefalopatias focais ou generalizadas (tumores, acidentes vásculo-encefálicos, meningite, encefalite);

> Traumatismo craniano, epilepsia não-tratada, indivíduos com parentes de primeiro grau com epilepsia idiopática;

$>$ Pacientes com uso inicial recente, $\leq 30$ dias de medicações que reduzem o limiar convulsivo (antidepressivos tricíclicos e antipsicóticos);

$>\quad$ Indivíduos em uso crônico de álcool ou drogas epileptogênicas (ex. cocaína);

> Cardiopatias graves, clipes neurocirúrgicos, marca-passo cardíaco, aumento de pressão intracraniana (risco de sequelas após convulsão);

> Mulheres grávidas ou amamentando.

\subsection{Protocolo de avaliação e tratamento}

Após a seleção e randomização, os indivíduos foram encaminhados para um protocolo de avaliação realizado por um profissional treinado e habilitado, que não teve acesso aos parâmetros de EMT e era cego quanto ao grupo a que pertencia o doente (ativo, placebo ou controle). As avaliações e o tratamento dos doentes foram realizados de acordo com o roteiro da Figura 4. Os controles foram avaliados somente quanto à excitabilidade cortical, ou seja, não realizaram a sessão de EMT mas 
somente as medidas de excitabilidade cortical para comparação destes parâmetros com os doentes tratados.

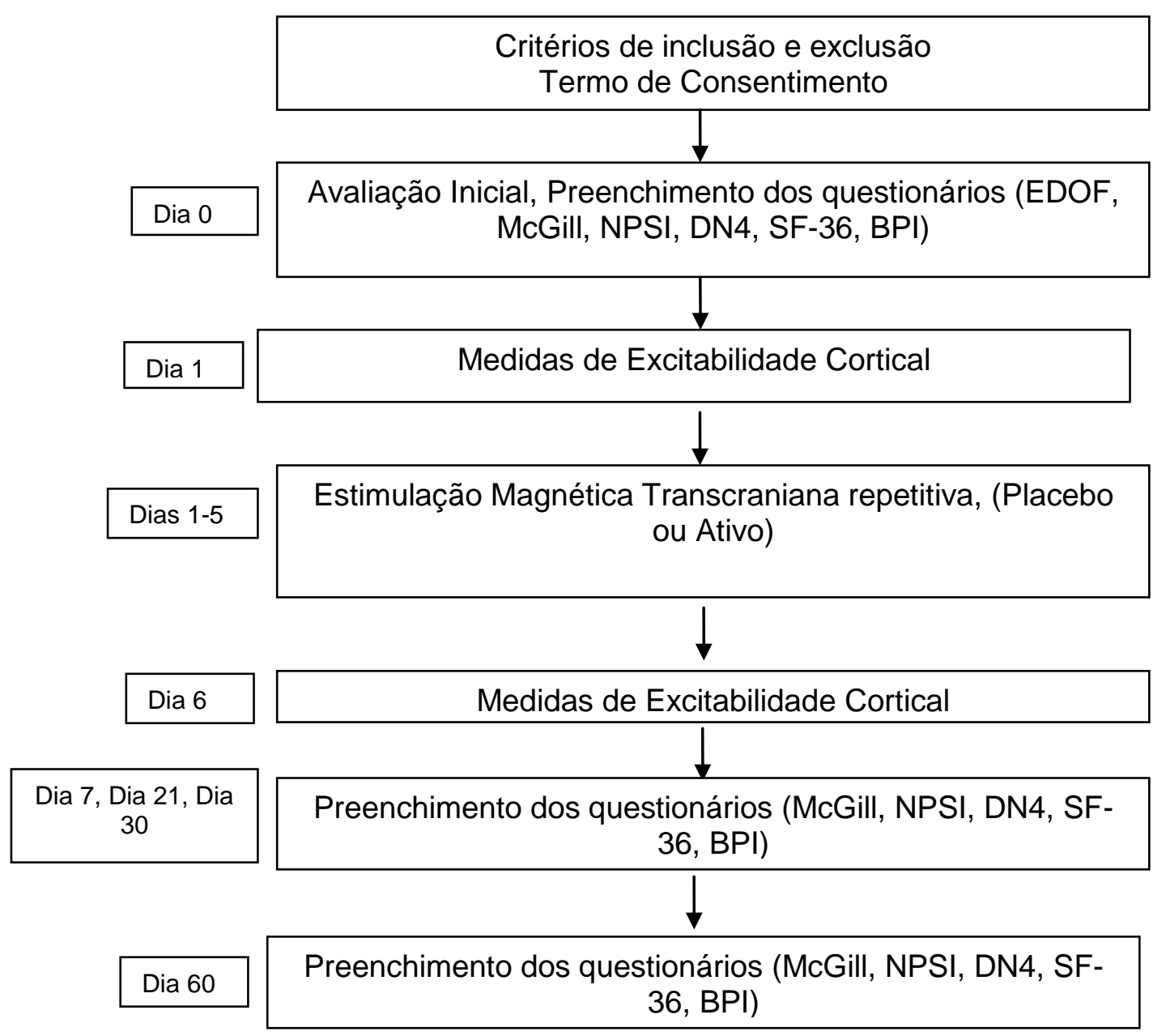

Figura 4 - Cronograma e roteiro de avaliação duplo-cega dos doentes

EDOF: Ficha clínica da equipe de dor orofacial; McGill: Questionário de dor McGill; NPSI: Inventário de Sintomas de Dor Neuropática; DN4: questionário para diagnóstico de dor neuropática; Sf-36: Inventário de qualidade de vida; BPI: Inventário Breve de Dor. 


\subsubsection{Instrumentos de avaliação}

4.2.1.1 Ficha clínica da equipe de dor orofacial (EDOF) e ATM (Divisão de Odontologia do HCFMUSP) para o diagnóstico de dor orofacial, que inclui: queixa principal, características principais de dor (localização, qualidade, duração, fatores de alívio, fatores de piora, intensidade pela EVA, cefaleia, dor generalizada, morbidades associadas e história médica (Siqueira et al., 2004) (ANEXO 4);

4.2.1.2 Questionário Breve de Dor McGill - validado para a língua portuguesa (Ferreira, de Andrade et al., 2011; Menezes Costa Lda, Maher et al., 2011), constituído de quinze itens para o domínio sensitivo, com escore máximo de 33 pontos e quatro para o domínio afetivo, com escore máximo de doze pontos); utilizado para a investigação dos descritores e índices de dor com escore total de 45 pontos (ANEXO 5);

\subsubsection{Inventário de Sintomas de Dor Neuropática (NPSI) - (de} Andrade, Ferreira et al., 2011) validado para a língua portuguesa. Trata-se de instrumento capaz de avaliar características da dor (dimensões), tanto centrais quanto periféricas, assim como o efeito do tratamento para as dores neuropáticas; tem escore máximo de cem pontos (ANEXO $6)$; 


\subsubsection{Questionário para diagnóstico de Dor Neuropática-DN4 -} (Santos, Brito et al., 2010) validado para a língua portuguesa. Trata-se de instrumento simples e objetivo que tem a propriedade de distinguir a dor neuropática da dor nociceptiva; considera-se a presença de dor neuropática o escore maior ou igual a quatro pontos em dez (ANEXO 7);

4.2.1.5 Inventário de Qualidade de Vida SF-36 - (Ciconelli, et al., 1999) traduzido e validado para a língua portuguesa. Tratase de instrumento genérico de fácil aplicação e compreensão usado para avaliação da qualidade de vida. Multidimensional, engloba oito domínios: capacidade funcional, aspecto físico, dor, estado geral de saúde, vitalidade, aspectos sociais e emocionais e saúde mental; o escore de cada domínio varia de zero a cem pontos (ANEXO 8);

4.2.1.6 Inventário Breve de Dor (BPI) - (Ferreira, Teixeira et al., 2011) validado para a língua portuguesa. Trata-se de instrumento breve e de fácil compreensão que avalia o quanto a dor interfere no cotidiano do paciente. Avalia a eficácia do tratamento, intensidade e local da dor; pode ser dividido em duas partes: a primeira refere-se à média de dor, com pontuação de zero a dez; a segunda refere-se à interferência da dor no cotidiano com escore de zero a setenta pontos (ANEXO 9). 


\subsection{Procedimento da EMT}

\subsubsection{Medidas de excitabilidade cortical (grupos de estudo e controle)}

Conforme descrito por Mhalla et al. (2010), doentes e controles foram submetidos a uma sessão inicial de 45 minutos usando-se uma bobina circular direcionada tangencialmente ao escalpo, na posição anteroposterior, na qual foram verificadas a excitabilidade cortical motora, o limiar motor de repouso, o potencial motor evocado e a facilitação e inibição cortical para cada hemisfério. Entende-se por limiar motor a menor intensidade de estímulo que provoque um potencial motor de $50 \mu \mathrm{V}$ em $50 \%$ das tentativas.

O intuito dessa fase do protocolo foi avaliar a relação entre intensidade e amplitude do estímulo (curva de resposta). O potencial motor evocado deu-se a $120 \%$ e $140 \%$ do limiar motor de repouso em masseter contralateral (Cruccu, Frisardi et al., 1997; McMillan, Graven-Nielsen et al., 2001; Pearce, Miles et al., 2003; Nordstrom, 2007), e a amplitude foi calculada a partir dessa relação (140/120). Utilizando-se dois pulsos pareados com intensidade de $80 \%$ do limiar motor de repouso para o pulso condicionante, e intensidade de $120 \%$ para o pulso teste. A pesquisa foi realizada com intervalos interestímulos de 2 e 4 ms para a inibição intracortical de intervalo curto, e com intervalos de 10 e 15 ms para a facilitação cortical. 


\subsubsection{Tratamento com EMTr (Ativo ou Placebo) (grupos de estudo)}

Os doentes com DFA foram distribuídos de forma aleatorizada para a estimulação do córtex motor primário contralateral ao lado da dor em dois grupos: ativo ou placebo (EMTr). Foi utilizado o seguinte protocolo: 3000 pulsos, com valor de $80 \%$ do limiar motor, disparados em 30 trens de 20 segundos a $10 \mathrm{~Hz}$ (frequência excitatória), com intervalos de 20 segundos entre os trens. Tal protocolo é semelhante ao utilizado pelo Centro Interdisciplinar de Dor do Departamento de Neurologia do HCFMUSP para investigar o alívio da dor, conforme descrito anteriormente.

Na Figura 5, é apresentado o modelo da máquina que foi utilizada para a EMTr e a excitabilidade cortical.

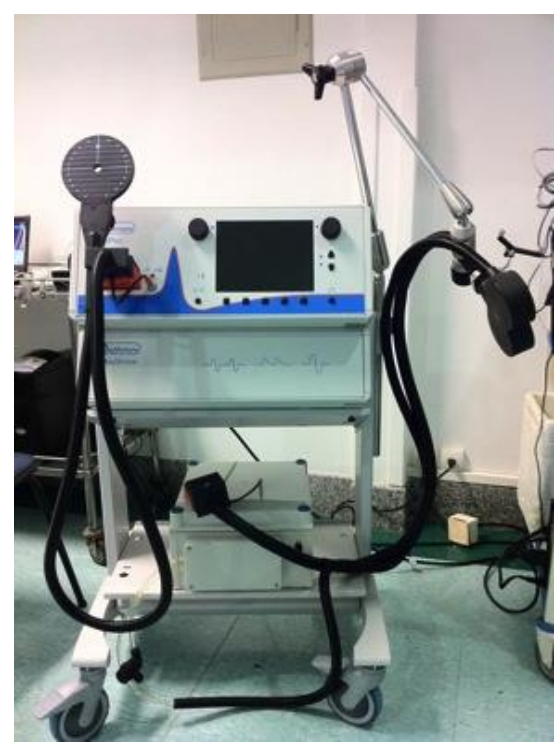

Figura 5 - Modelo de máquina de EMTr utilizada no estudo. MagProX100 ${ }^{\circledR}$ (MagVenture®)Tonika Elektronic, Farum, Dinamarca)

O estimulador pode ser dividido em três sistemas: 1) circuito de carga, 2) circuito de saída e 3) bobina propriamente dita. Esses estimuladores tem 
capacidade de armazenar carga em um reservatório que é permanentemente carregado ao máximo de 300 joules (Magventure Tonika Elektronic, 2009). No circuito de saída, há um interruptor acionado para descarregar em poucos microssegundos o reservatório para a bobina; a quantidade máxima de energia aproximada liberada na bobina é $180 \mathrm{~A} / \mu \mathrm{s}$. Essa por si só é um gerador de campo magnético, convertendo energia recebida do reservatório em um campo magnético que pode chegar a 4.1 Tesla, dependendo da especificação da bobina.

No presente estudo, foram usados três tipos de bobina: a) bobina refrigerada em figura de oito $\mathrm{BC}-65^{\circledR}$ (Magventure Tonika, Elektronic, Farum, Dinamarca) para o grupo ativo, que permite que o campo magnético seja multiplicado, e o volume estimulado na ordem de $6 \mathrm{~cm}^{2}$; b) bobina em figura de oito $B C-70^{\circledR}$ (Magventure Tonika, Elektronic, Farum, Dinamarca) para o grupo placebo, com aparência similar à bobina $\mathrm{BC}-65^{\circledR}$, porém com blindagem que não permite a passagem do campo magnético. $O$ doente ouve o barulho da estimulação mas não recebe seus efeitos; c) bobina circular C-100, conectada a um módulo amplificador de eletroneuromiografia (EMG) (Magventure Tonika Elektronic, Farum, Dinamarca) com saída para três eletrodos de superfície (1 canal) (Alpine Biom, Skovlunde, Dinamarca), para potenciais evocados motores e medida de excitabilidade cortical; possui diâmetro de 8 a $10 \mathrm{~cm}$ e campo eletromagnético mais difuso, sendo esse maior nas extremidades da bobina e quase zero no centro. A Figura 6 demonstra os diferentes campos eletromagnéticos gerados pelas bobinas. 


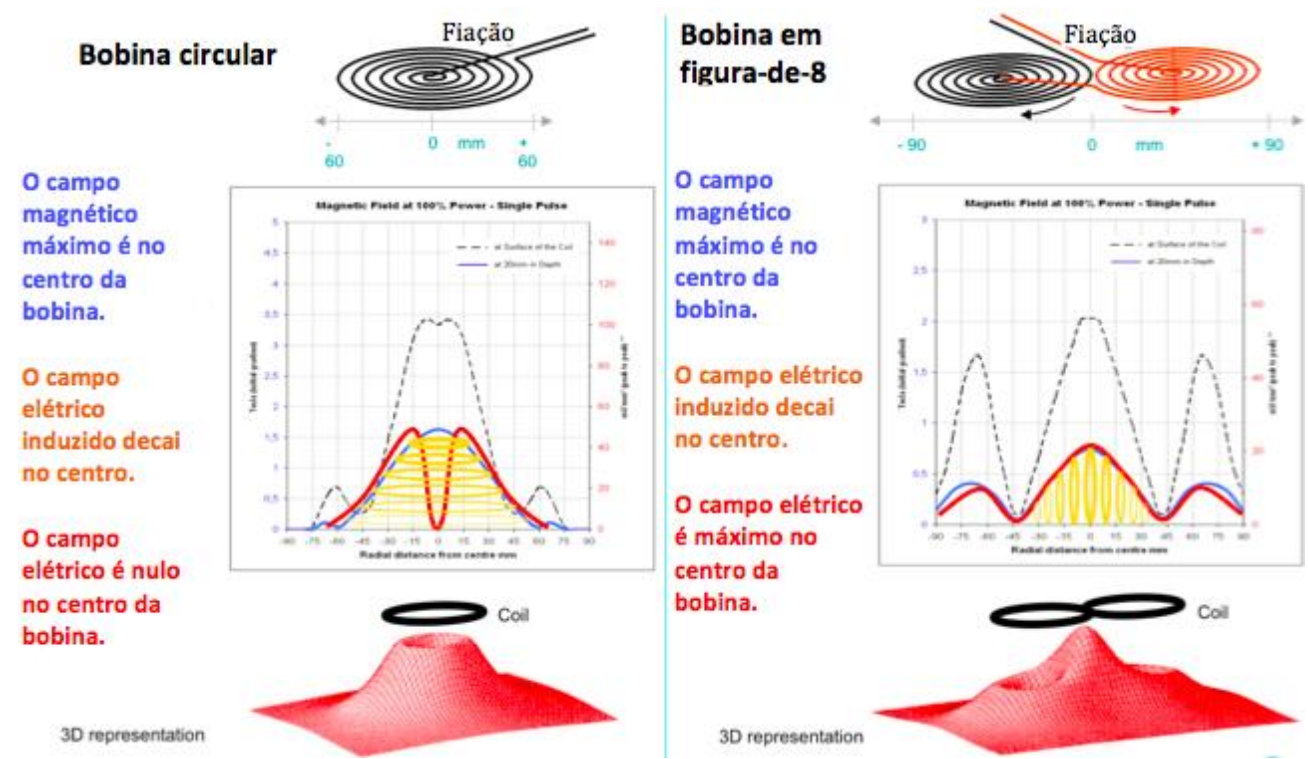

Figura 6 - Diferenças entre os campos gerados pela bobina circular (esquema esquerda) e bobina figura de oito (esquema direita).

Fonte: (Magventure ${ }^{\circledR}$, Dinamarca, 2010).

\subsection{Análise dos dados}

As variáveis foram descritas através de médias, desvios padrão, medianas, variações, frequências e porcentagens; as variáveis quantitativas foram testadas quanto à distribuição normal através dos testes KolmogorovSmirnoff e gráficos Q-Q. Inicialmente, foram utilizados os testes ANOVA 1 fator (paramétrico) e Mann-Whitney (não paramétrico). O teste de Quiquadrado foi utilizado para as variáveis qualitativas nominais e ordinais. Comparação de efeito entre os diferentes tempos de avaliação foi analisada através de ANOVA de medidas repetidas. Correlação múltipla de dados clínicos e eletrofisiológicos foi analisada pelo coeficiente de Bonferroni. Análise de sobrevida utilizando a curva de Kaplan-Meier foi utilizada para a investigação estatística da melhora da dor. Ela inclui o teste log-rank para 
verificar diferenças entre grupos, e seu cálculo considera o número total dos doentes que apresentaram melhora dividido pelos demais. Doentes que perderam o seguimento são censurados (Perera, Heneghan et al., 2010). O nível de significância foi de 5\%. 
5. RESULTADOS (5. RESULTADOS 


\subsection{Avaliação inicial (Pré-tratamento)}

\subsubsection{Características gerais dos doentes}

A comparação entre os grupos pode ser observada na Tabela 5. Não houve diferenças estatísticas quanto a gênero, idade, altura, peso, etnia, estado civil, renda, atividade laboral, religião e tabagismo. A morbidade associada mais prevalente foi hipertensão arterial sistêmica (31\%), sendo que não houve diferença entre os grupos. A maioria dos doentes classificou sua saúde como boa $(41,4 \%)$ ou ruim $(37,9 \%)$ (Tabela 7$)$. 
Tabela 5 - Características gerais de acordo com o grupo de doentes em números absolutos (N), porcentagens (\%), médias e desvio padrão, e resultados dos testes de associação (p) $(\mathrm{N}=29)$

\begin{tabular}{|c|c|c|c|c|c|c|c|c|}
\hline \multirow{3}{*}{$\begin{array}{l}\text { Características } \\
\text { Idade (em anos) }^{\#}\end{array}$} & \multirow{3}{*}{ Categorias } & \multicolumn{4}{|c|}{ Grupos } & \multirow{2}{*}{\multicolumn{2}{|c|}{ Total }} & \multirow[b]{2}{*}{ p } \\
\hline & & \multicolumn{2}{|c|}{$\begin{array}{l}\text { Grupo1 (Ativo) } \\
(\mathrm{N}=15)\end{array}$} & \multicolumn{2}{|c|}{$\begin{array}{c}\text { Grupo } 2 \text { (Placebo) } \\
(\mathrm{N}=14)\end{array}$} & & & \\
\hline & & \multicolumn{2}{|c|}{$\begin{array}{c}55,60 \pm 11,12 \\
(35-74)\end{array}$} & \multicolumn{2}{|c|}{$\begin{array}{c}56,0 \pm 13,59 \\
(28-77) \\
\end{array}$} & \multicolumn{2}{|c|}{$\begin{array}{c}53,28 \pm 12,39 \\
(28-78) \\
\end{array}$} & 0,780 \\
\hline \multicolumn{2}{|l|}{$\begin{array}{l}\text { Peso } \\
\text { (em quilogramas) }^{\#}\end{array}$} & \multicolumn{2}{|c|}{$\begin{array}{c}67,13 \pm 11,59 \\
(51-86) \\
\end{array}$} & \multicolumn{2}{|c|}{$\begin{array}{c}67,64 \pm 11,98 \\
(43-90)\end{array}$} & \multicolumn{2}{|c|}{$\begin{array}{c}67,37 \pm 11,57 \\
(43-90)\end{array}$} & 0,780 \\
\hline \multicolumn{2}{|l|}{$\begin{array}{l}\text { Altura } \\
\text { (em centímetros) }\end{array}$} & \multicolumn{2}{|c|}{$\begin{array}{c}1,60 \pm 0,06 \\
(1,50-1,70)\end{array}$} & \multicolumn{2}{|c|}{$\begin{array}{c}1,63 \pm 0,06 \\
(1,52-1,72)\end{array}$} & \multicolumn{2}{|c|}{$\begin{array}{c}1,62 \pm 0,06 \\
(1,50-1,72)\end{array}$} & 0,252 \\
\hline \multirow[t]{2}{*}{$\begin{array}{l}\text { Renda familiar } \\
\text { (em reais) }\end{array}$} & & \multicolumn{2}{|c|}{$\begin{array}{c}2.613,33 \pm 2.151,69 \\
(700,00-8.000,00)\end{array}$} & \multicolumn{2}{|c|}{$\begin{array}{c}2.407,14 \pm 1.637,75 \\
(800,00-6.000,00)\end{array}$} & \multicolumn{2}{|c|}{$\begin{array}{c}2.513,79 \pm 1.889,77 \\
(700,00-8.000,00)\end{array}$} & 0,847 \\
\hline & & $\mathrm{N}$ & $\%$ & $\mathrm{~N}$ & $\%$ & $\mathrm{~N}$ & $\%$ & \\
\hline \multirow[t]{5}{*}{ Etnia $^{\star \star}$} & Negro & 2 & 13,3 & 1 & 7,1 & 3 & 10,3 & 0,949 \\
\hline & Branca & 13 & 86,7 & 12 & 85,8 & 25 & 86,3 & \\
\hline & Parda & 0 & 0 & 1 & 7,1 & 1 & 3,4 & \\
\hline & Amarela & 0 & 0 & 0 & 0 & 0 & 0 & \\
\hline & Total & 15 & 100 & 14 & 100 & 29 & 100 & \\
\hline Estado civil $^{\star \star}$ & Casado & 11 & 73,3 & 9 & 64,3 & 20 & 68,9 & 0,813 \\
\hline & Viúvo & 1 & 6,7 & 0 & 0 & 1 & 3,4 & \\
\hline & Divorciado & 0 & 0 & 1 & 7,1 & 1 & 3,4 & \\
\hline & Solteiro & 3 & 20,0 & 3 & 21,5 & 6 & 20,9 & \\
\hline & Outros & 0 & 0 & 1 & 7,1 & 1 & 3,4 & \\
\hline & Total & 15 & 100 & 14 & 100 & 29 & 100 & \\
\hline Renda familiar (em & Menos de 1 & 0 & 0 & 0 & 0 & 0 & 0 & 0,780 \\
\hline salário mínimos) $^{\star *}$ & De 1 a 2 & 7 & 46,7 & 8 & 57,4 & 15 & 51,8 & \\
\hline & De 3 a 5 & 6 & 40,0 & 3 & 21,3 & 9 & 31,0 & \\
\hline & De 6 a 10 & 1 & 6,7 & 3 & 21,3 & 4 & 13,8 & \\
\hline & Mais de10 & 1 & 6,7 & 0 & 0 & 1 & 3,4 & \\
\hline & Total & 15 & 100,0 & 14 & 100 & 29 & 100 & \\
\hline Atividade laboral $^{\star \star}$ & Aposentado & 4 & 26,7 & 4 & 28,6 & 8 & 27,6 & 0,290 \\
\hline & $\begin{array}{l}\text { Trabalho } \\
\text { doméstico }\end{array}$ & 4 & 26,7 & 2 & 14,3 & 6 & 20,8 & \\
\hline & $\begin{array}{l}\text { Trabalho } \\
\text { externo }\end{array}$ & 6 & 40,0 & 8 & 57,1 & 14 & 48,2 & \\
\hline & $\begin{array}{l}\text { Licença } \\
\text { médica }\end{array}$ & 1 & 6,7 & 0 & 0 & 1 & 3,4 & \\
\hline & Total & 15 & 100,0 & 14 & 100,0 & 29 & 100 & \\
\hline Escolaridade ${ }^{\star \star}$ & $\begin{array}{l}\text { Ensino } \\
\text { Fundamental }\end{array}$ & 9 & 60,0 & 5 & 35,7 & 14 & 48,2 & 0,650 \\
\hline & $\begin{array}{l}\text { Ensino } \\
\text { Médio } \\
\end{array}$ & 4 & 26,7 & 4 & 28,6 & 8 & 27,6 & \\
\hline & $\begin{array}{l}\text { Superior ou } \\
\text { mais }\end{array}$ & 2 & 13,3 & 4 & 28,5 & 6 & 20,8 & \\
\hline & $\begin{array}{l}\text { Sem } \\
\text { resposta }\end{array}$ & 0 & 0 & 1 & 7,1 & 1 & 3,4 & \\
\hline & Total & 15 & 100,0 & 14 & 100,0 & 29 & 100 & \\
\hline Religião** & Sem religião & 0 & 0 & 2 & 14,3 & 2 & 6,9 & 0,715 \\
\hline & Católico & 11 & 73,3 & 7 & 50,0 & 18 & 62,1 & \\
\hline & Evangélico & 2 & 13,3 & 2 & 14,3 & 4 & 13,8 & \\
\hline & Outras & 2 & 13,3 & 3 & 21,4 & 5 & 17,2 & \\
\hline & Total & 15 & 100,0 & 14 & 100,0 & 29 & 100 & \\
\hline Tabagismo*** & Sim & 3 & 20,0 & 1 & 7,1 & 4 & 13,8 & 0,561 \\
\hline & Não & 12 & 80,0 & 13 & 92,9 & 25 & 86,2 & \\
\hline & Total & 15 & 100,0 & 14 & 100 & 29 & 100 & \\
\hline Etilismo** & Sim & 0 & 0 & 0 & 0 & 0 & 0 & 1,000 \\
\hline & Não & 15 & 100,0 & 14 & 100 & 29 & 100 & \\
\hline
\end{tabular}


${ }^{* *}$ Teste qui-quadrado; ***Teste Exato de Fisher; \#Resultado expresso no formato média \pm desvio-padrão (variação).

Tabela 6 - Morbidades associadas de acordo com o grupo em números absolutos (N), porcentagens (\%) e resultados dos testes de associação (p) ( $N=29)$

\begin{tabular}{|c|c|c|c|c|c|c|c|}
\hline \multirow[t]{3}{*}{ 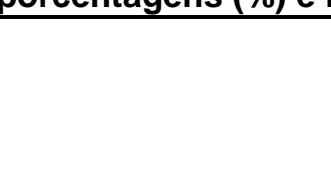 } & \multicolumn{4}{|c|}{ Grupos* } & \multirow[b]{3}{*}{ Total } & & \multirow[b]{4}{*}{$\mathbf{p}^{\star \star}$} \\
\hline & \multirow{2}{*}{\multicolumn{2}{|c|}{$\begin{array}{l}\text { Grupo } 1 \text { (Ativo) } \\
(\mathrm{N}=15)\end{array}$}} & \multicolumn{2}{|c|}{ Grupo 2 (Placebo) } & & & \\
\hline & & & $(\mathrm{N}=1$ & & & & \\
\hline Doenças & $\mathbf{N}$ & $\%$ & $\overline{\mathbf{N}}$ & $\%$ & $\mathbf{N}$ & $\%$ & \\
\hline Amigdalite & 1 & 6,7 & 1 & 7,1 & 2 & 6,9 & 0,741 \\
\hline Artrite reumatoide & 1 & 6,7 & 1 & 7,1 & 2 & 6,9 & 0,741 \\
\hline Asma & 1 & 6,7 & 1 & 7,1 & 2 & 6,9 & 0,741 \\
\hline Bronquite & 1 & 6,1 & 1 & 7,1 & 2 & 6,9 & 0,741 \\
\hline Depressão & 3 & 20,0 & 4 & 28,6 & 7 & 24,1 & 0,458 \\
\hline Diabetes & 1 & 6,7 & 1 & 7,1 & 2 & 6,9 & 0,741 \\
\hline Doença Cardíaca & 1 & 6,7 & 0 & 0 & 1 & 3,4 & 0,517 \\
\hline Doença Renal & 0 & 0 & 1 & 7,1 & 1 & 3,4 & 0,483 \\
\hline Enxaqueca & 2 & 13,3 & 4 & 28,6 & 6 & 20,7 & 0,291 \\
\hline Fibromialgia & 1 & 6,7 & 1 & 7,1 & 2 & 6,9 & 0,741 \\
\hline Gastrite & 5 & 33,3 & 3 & 21,4 & 8 & 27,6 & 0,383 \\
\hline HAS & 6 & 40,0 & 3 & 21,4 & 9 & 31,0 & 0,250 \\
\hline Hepatite & 2 & 13,3 & 0 & 0 & 2 & 6,9 & 0,259 \\
\hline Herpes Zoster & 1 & 6,7 & 0 & 0 & 1 & 3,4 & 0,517 \\
\hline Rinite alérgica & 3 & 20,0 & 3 & 21,4 & 6 & 20,7 & 0,639 \\
\hline Sinusite & 3 & 20,0 & 5 & 35,7 & 8 & 27,6 & 0,298 \\
\hline Úlcera & 0 & 0 & 2 & 14,3 & 2 & 6,9 & 0,224 \\
\hline Outras & 6 & 40,0 & 3 & 21,4 & 9 & 31,0 & 0,250 \\
\hline
\end{tabular}

**Teste exato de Fisher. 
Tabela 7 - Autoavaliação da saúde de acordo com o grupo em números absolutos (N), porcentagens (\%) e resultados dos testes de associação (p) ( $N=29)$

\begin{tabular}{|c|c|c|c|c|c|c|c|}
\hline & \multicolumn{4}{|c|}{ Grupos* } & \multirow[b]{3}{*}{ Total } & & \multirow[b]{4}{*}{$p^{\star \star}$} \\
\hline & \multirow{2}{*}{\multicolumn{2}{|c|}{$\begin{array}{l}\text { Grupo1 (Ativo) } \\
(\mathrm{N}=15)\end{array}$}} & \multirow{2}{*}{\multicolumn{2}{|c|}{$\begin{array}{l}\text { Grupo } 2 \text { (Placebo) } \\
(\mathrm{N}=14)\end{array}$}} & & & \\
\hline & & & & & & & \\
\hline Avaliação & $\mathbf{N}$ & $\%$ & $\mathbf{N}$ & $\%$ & $\mathbf{N}$ & $\%$ & \\
\hline Excelente & 0 & 0 & 0 & 0 & 0 & 0 & 0,708 \\
\hline Muito boa & 1 & 6,7 & 1 & 7,1 & 2 & 6,9 & \\
\hline Boa & 7 & 46,7 & 5 & 35,7 & 12 & 41,4 & \\
\hline Ruim & 6 & 40,0 & 5 & 35,7 & 11 & 37,9 & \\
\hline Muito & 1 & 6,7 & 3 & 21,4 & 4 & 13,8 & \\
\hline Ruim & & & & & & & \\
\hline Total & 15 & 100 & 14 & 100 & 29 & 100 & \\
\hline
\end{tabular}

**Teste do qui-quadrado.

\subsubsection{Características odontológicas, características álgicas e excitabilidade cortical}

Não houve diferenças estatísticas com relação a edentulismo, perda de dimensão vertical, ausências dentárias, características de mordida e frequência de periodontite (Tabela 8). O Grupo 1 (EMTr Ativo) apresentou maior tempo de uso de próteses totais $(p=0,031)$ e menor frequência de desgastes dentários $(p=0,015)$.

O lado mais afetado pela dor foi o esquerdo $(55,2 \%)$, sendo que não houve diferenças entre os grupos com relação a fatores desencadeantes, de piora, de melhora e tratamentos prévios (Tabela 9). 
Tabela 8 - Características odontológicas de acordo com o grupo em números absolutos (N), porcentagens (\%) e resultados dos testes de associação (p) (N=29)

\begin{tabular}{|c|c|c|c|c|c|}
\hline \multirow[b]{2}{*}{ Características } & \multirow[b]{2}{*}{ Categorias } & \multicolumn{2}{|c|}{ Grupos* } & \multirow[b]{2}{*}{ Total } & \multirow[b]{2}{*}{$\mathbf{p}$} \\
\hline & & $\begin{array}{c}\begin{array}{c}\text { Grupo } 1 \\
\text { (Ativo) }\end{array} \\
(\mathrm{N}=15)\end{array}$ & $\begin{array}{c}\begin{array}{c}\text { Grupo 2 } \\
\text { (Placebo) }\end{array} \\
(\mathrm{N}=14)\end{array}$ & & \\
\hline \multirow[t]{5}{*}{ Edêntulos ${ }^{\star *}$} & Superior & $3(20,0 \%)$ & $4(28,6 \%)$ & $7(24,1 \%)$ & 0,781 \\
\hline & Inferior & 0 & 0 & 0 & \\
\hline & Duplo & $2(13,3 \%)$ & $1(7,1 \%)$ & $3(10,3 \%)$ & \\
\hline & $\begin{array}{l}\text { Parcial com } \\
\text { PPR }\end{array}$ & $5(33,7)$ & $2(14,3 \%)$ & $7(24,1 \%)$ & \\
\hline & Não edêntulos & $10(66,7 \%)$ & $9(64,3 \%)$ & $19(65,5 \%)$ & \\
\hline $\begin{array}{l}\text { Perda de dimensão } \\
\text { vertical }^{+\star \star x}\end{array}$ & & $1(6,7 \%)$ & $3(21,4 \%)$ & $4(13,8 \%)$ & 0,272 \\
\hline \multirow{2}{*}{$\begin{array}{l}\text { Tempo de uso de } \\
\text { prótese (em anos) }\end{array}$} & PT total & $28 \pm 11,66$ & $8 \pm 8,1$ & $18,0 \pm 14,1$ & 0,031 \\
\hline & $\begin{array}{l}\text { PT atual } \\
\text { PPR }\end{array}$ & $\begin{array}{c}5,25 \pm 4,7 \\
7 \pm 10,0\end{array}$ & $2,5 \pm 1,0$ & $\begin{array}{c}3,9 \pm 3,4 \\
6,20+8,9\end{array}$ & $\begin{array}{l}0,297 \\
0,747\end{array}$ \\
\hline \multirow[t]{4}{*}{$\begin{array}{l}\text { Ausências dentárias } \\
\text { (em no de dentes) }{ }^{\#}\end{array}$} & $\begin{array}{l}\text { Superior } \\
\text { homolateral }\end{array}$ & $3,5 \pm 3,07$ & $2,85 \pm 3,13$ & $3,18 \pm 3,06$ & 0,574 \\
\hline & $\begin{array}{l}\text { Inferior } \\
\text { homolateral }\end{array}$ & $3,07 \pm 3,09$ & $3,07 \pm 2,78$ & $3,07 \pm 2,89$ & 1,000 \\
\hline & $\begin{array}{l}\text { Superior } \\
\text { contralateral }\end{array}$ & $3,3 \pm 3,11$ & $2,64 \pm 3,17$ & $2,96 \pm 3,10$ & 0,589 \\
\hline & $\begin{array}{l}\text { Inferior } \\
\text { contralateral }\end{array}$ & $2,92 \pm 2,94$ & $2,64 \pm 2,97$ & $2,78 \pm 2,91$ & 0,801 \\
\hline \multirow{4}{*}{$\begin{array}{l}\text { Classificação de } \\
\text { Angle }^{\star \star}\end{array}$} & 1 & $15(100 \%)$ & 14 & $29(100 \%)$ & 1,000 \\
\hline & ॥ & 0 & 0 & 0 & \\
\hline & III & 0 & 0 & 0 & \\
\hline & Total & $15(100 \%)$ & 14 & $29(100 \%)$ & \\
\hline \multirow[t]{3}{*}{ Mordida } & Aberta & $1(6,7 \%)$ & 0 & $1(3,4 \%)$ & 0,517 \\
\hline & $\begin{array}{l}\text { Sobremordida } \\
\text { profunda }\end{array}$ & $1(6,7 \%)$ & $4(28,6 \%)$ & $5(17,2 \%)$ & 0,060 \\
\hline & Total & $2(13,4 \%)$ & $4(28,6 \%)$ & $6(20,6 \%)$ & \\
\hline \multirow[t]{4}{*}{ Desgaste dentário** } & Incisal & 0 & $5(35,7 \%)$ & $5(17,2 \%)$ & 0,015 \\
\hline & $1 / 3$ incisal & 3 & 0 & $3(10,3 \%)$ & \\
\hline & 1/3 médio & 0 & 0 & 0 & \\
\hline & Sem desgaste & $12(80,0 \%)$ & $9(64,3 \%)$ & $21(72,4 \%)$ & \\
\hline Periodontite & & $2(13,3 \%)$ & $4(28,6)$ & $6(20,7 \%)$ & 0,291 \\
\hline
\end{tabular}

** Teste do qui-quadrado; ***Teste exato de Fisher; \#Resultado expresso no formato média \pm Desvio padrão(variação), $p$ obtido através do teste ANOVA; PPR: prótese parcial removível; PT: prótese total. 
Tabela 9 - Características álgicas de acordo com o grupo em números absolutos (N), porcentagens (\%), médias e desvio padrão, e resultados dos testes de associação (p) $(\mathrm{N}=29)$

\begin{tabular}{|c|c|c|c|c|c|c|c|c|}
\hline \multirow[b]{2}{*}{ Características } & \multirow[b]{2}{*}{ Categorias } & \multicolumn{4}{|c|}{ Grupos* } & \multirow[b]{2}{*}{ Total } & & \multirow[b]{2}{*}{$\mathbf{P}^{*}$} \\
\hline & & $\begin{array}{c}\begin{array}{c}\text { Grupo } 1 \\
\text { (Ativo) }\end{array} \\
(\mathrm{N}=15)\end{array}$ & & $\begin{array}{c}\begin{array}{c}\text { Grupo } 2 \\
\text { (Placebo) }\end{array} \\
(\mathrm{N}=14)\end{array}$ & & & & \\
\hline \multirow[t]{2}{*}{$\begin{array}{l}\begin{array}{l}\text { Tempo de dor } \\
\text { (em meses)\# }\end{array} \\
\end{array}$} & & $\begin{array}{c}14,3 \pm 12 \\
20 \\
\end{array}$ & & $\begin{array}{c}12,6 \pm 10,0 \\
4\end{array}$ & & $\begin{array}{c}13,5 \pm 1 \\
1,04 \\
\end{array}$ & & 0,700 \\
\hline & & $\mathrm{N}$ & $\%$ & $\mathrm{~N}$ & $\%$ & $\mathrm{~N}$ & $\%$ & \\
\hline \multirow[t]{3}{*}{ Lado da dor } & Esquerdo & 9 & 60 & 7 & 50 & 16 & 55,2 & 0,599 \\
\hline & Direito & 2 & 13,3 & 4 & 28,6 & 6 & 20,7 & \\
\hline & Bilateral & 4 & 26,7 & 3 & 21,4 & 7 & 24,1 & \\
\hline \multirow[t]{5}{*}{$\begin{array}{l}\text { Fator } \\
\text { desencadeante }\end{array}$} & $\begin{array}{l}\text { Tratamento } \\
\text { Odontológico }\end{array}$ & 5 & 33,3 & 5 & 35,7 & 10 & 34,5 & 0,625 \\
\hline & $\begin{array}{l}\text { Tratamento de } \\
\text { saúde }\end{array}$ & 2 & 13,3 & 0 & 0 & 2 & 6,9 & \\
\hline & Estresse & 3 & 20,0 & 2 & 14,3 & 5 & 17,2 & \\
\hline & Acidente & 1 & 6,7 & 2 & 13,4 & 3 & 10,3 & \\
\hline & Não sabe & 4 & 26,7 & 5 & 35,7 & 9 & 31 & \\
\hline \multirow[t]{5}{*}{ Fator de piora } & Estresse & 2 & 13,3 & 7 & 50 & 9 & 31 & 0,136 \\
\hline & $\begin{array}{l}\text { Mudança } \\
\text { temperatura } \\
\text { (frio/quente) }\end{array}$ & 5 & 33,3 & 4 & 28,6 & 9 & 31 & \\
\hline & $\begin{array}{l}\text { Toque região } \\
\text { face }\end{array}$ & 4 & 26,7 & 3 & 21,4 & 7 & 24,1 & \\
\hline & Alimentação & 1 & 6,7 & 0 & 0 & 1 & 3,4 & \\
\hline & Nenhum & 3 & 20,0 & 0 & 0 & 3 & 10,3 & \\
\hline \multirow{4}{*}{$\begin{array}{l}\text { Fatores de } \\
\text { melhora }\end{array}$} & Medicação & 4 & 26,7 & 7 & 50 & 11 & 37,9 & 0,327 \\
\hline & Relaxar & 5 & 33,3 & 5 & 35,7 & 10 & 34,5 & \\
\hline & Outros & 1 & 6,7 & 1 & 7,1 & 2 & 6,9 & \\
\hline & Nenhum & 5 & 33,3 & 1 & 7,1 & 6 & 20,7 & \\
\hline \multirow[t]{6}{*}{$\begin{array}{l}\text { Tratamentos } \\
\text { prévios }\end{array}$} & Medicamento & 3 & 20,0 & 5 & 35,7 & 8 & 27,6 & 0,526 \\
\hline & Exodontia & 1 & 6,7 & 1 & 7,1 & 2 & 6,9 & \\
\hline & Cirúrgico & 1 & 6,7 & 0 & 0 & 1 & 3,4 & \\
\hline & $\begin{array}{l}\text { Tratamentos } \\
\text { concomitantes ** }\end{array}$ & 8 & 53,3 & 5 & 35,7 & 13 & 44,8 & \\
\hline & Outros & 1 & 6,7 & 3 & 21,4 & 4 & 13,8 & \\
\hline & Nenhum & 1 & 6,7 & 0 & 0 & 1 & 3,4 & \\
\hline
\end{tabular}

*Teste do qui-quadrado; \# Resultado expresso no formato média \pm desvio-padrão (variação), $\mathrm{p}$ obtido através de ANOVA; ${ }^{*}$ Tratamentos concomitantes (medicamento+cirurgia ou medicamento+tratamento odontológico ou medicamento+outro tipo de terapia).

Pode-se observar na Tabela 10 que os doentes de ambos os grupos apresentaram características semelhantes quanto às características de dor pelo DN-4, NPSI e BPI, e quanto à qualidade de vida (SF-36). O Grupo 1 (Ativo) apresentou maior frequência de descritores sensitivos ( $P=0,031)$. 
Tabela 10 - Avaliação dos doentes através do DN4, NPSI, Questionário de Dor McGill, BPI e SF-36 de acordo com o grupo em médias e desvio padrão, e resultados dos testes de associação (p) ( $N=29)$

\begin{tabular}{|c|c|c|c|c|c|c|}
\hline \multirow[b]{3}{*}{ Características } & \multirow[b]{3}{*}{ Categorias } & \multicolumn{4}{|c|}{ Grupos* $^{*}$} & \multirow[b]{3}{*}{$\mathbf{p}$} \\
\hline & & \multicolumn{2}{|c|}{$\begin{array}{c}\text { Grupo } 1 \\
\text { (Ativo) }\end{array}$} & \multicolumn{2}{|c|}{$\begin{array}{c}\text { Grupo } 2 \\
\text { (Placebo) }\end{array}$} & \\
\hline & & $\mathrm{N}=15$ & DP & $\mathrm{N}=14$ & DP & \\
\hline DN4 $^{\star \star}$ & & 5,66 & $\pm 2,43$ & 4,35 & $\pm 2,20$ & 0,142 \\
\hline NPSI** & & 47,13 & 17,12 & 39,64 & 23,48 & 0,333 \\
\hline \multirow[t]{3}{*}{ McGill $^{\star \star \star}$} & Afetivo & 10,06 & $\pm 2,54$ & 7,64 & $\pm 4,10$ & 0,186 \\
\hline & Sensitivo** & 17,86 & $\pm 3,87$ & 12,85 & $\pm 7,53$ & 0,031 \\
\hline & Total $^{\star *}$ & 27,93 & $\pm 5,75$ & 20,50 & $\pm 10,65$ & 0,260 \\
\hline \multirow[t]{5}{*}{$\mathrm{BPI}^{\star \star}$} & Dor mais fraca & 5,2 & $\pm 2,4$ & 4,7 & $\pm 2,7$ & 0,620 \\
\hline & Média de dor & 7,1 & $\pm 2,1$ & 6,3 & $\pm 1,9$ & 0,312 \\
\hline & Dor mais forte & 8,3 & $\pm 1,6$ & 7,9 & $\pm 1,7$ & 0,553 \\
\hline & $\begin{array}{l}\text { Dor no } \\
\text { momento }\end{array}$ & 6,2 & $\pm 2,7$ & 5,5 & $\pm 3,4$ & 0,556 \\
\hline & Interferência & 34,13 & $\pm 20,92$ & 42,42 & 19,00 & 0,456 \\
\hline \multirow[t]{8}{*}{ SF-36 } & $\begin{array}{l}\text { Capacidade } \\
\text { funcional }\end{array}$ & 61,33 & $\pm 28,43$ & 66,42 & $\pm 27,55$ & 0,715 \\
\hline & $\begin{array}{l}\text { Aspectos } \\
\text { Físicos }\end{array}$ & 43,33 & $\pm 43,77$ & 39,28 & $\pm 45,69$ & 0,780 \\
\hline & $\begin{array}{l}\text { Estado geral de } \\
\text { saúde }^{\star \star}\end{array}$ & 60,40 & $\pm 13,36$ & 59,78 & $\pm 10,87$ & 0,893 \\
\hline & Vitalidade $^{\star *}$ & 48,66 & $\pm 24,74$ & 43,92 & $\pm 21,04$ & 0,893 \\
\hline & $\begin{array}{l}\text { Aspectos } \\
\text { sociais }\end{array}$ & 57,5 & $\pm 32,32$ & 42,85 & $\pm 32,41$ & 0,172 \\
\hline & $\begin{array}{l}\text { Aspectos } \\
\text { emocionais }\end{array}$ & 31,11 & $\pm 40,75$ & 33,33 & $\pm 39,22$ & 0,880 \\
\hline & Saúde mental** & 46,93 & $\pm 26,59$ & 45,42 & $\pm 24,83$ & 0,876 \\
\hline & Dor $^{\star *}$ & 37,80 & $\pm 21,50$ & 36,14 & $\pm 26,14$ & 0,853 \\
\hline
\end{tabular}

${ }^{* *}$ Teste $t$ de Student para amostras independentes; ${ }^{* * *}$ Teste $U$ de Mann-Whitney. McGill: Questionário de dor McGill; NPSI: Inventário de Sintomas de Dor Neuropática; DN4: questionário para diagnóstico de dor neuropática; Sf-36: Inventário de qualidade de vida; BPI: Inventário Breve de Dor.

Não houve assimetria entre os hemisférios direito e esquerdo. Portanto, para a comparação com o grupo controle (indivíduos saudáveis), realizou-se o agrupamento dos hemisférios no momento da avaliação basal para as medidas de inibição e facilitação intracortical. Foram apresentadas diferenças quanto à medida de excitabilidade cortical, para a amplitude do potencial evocado motor (PEM) a 120\% direito ( $p=0,025)$; PEM 140\% direito $(p=0,006)$ e PEM $120 \%$ esquerdo $(p=0,002)$, (Figura 8). Os pacientes 
apresentaram porcentagem de inibição e facilitação, respectivamente, maiores que os controles ( $p<0,001)$, (Figuras 9 e 10).

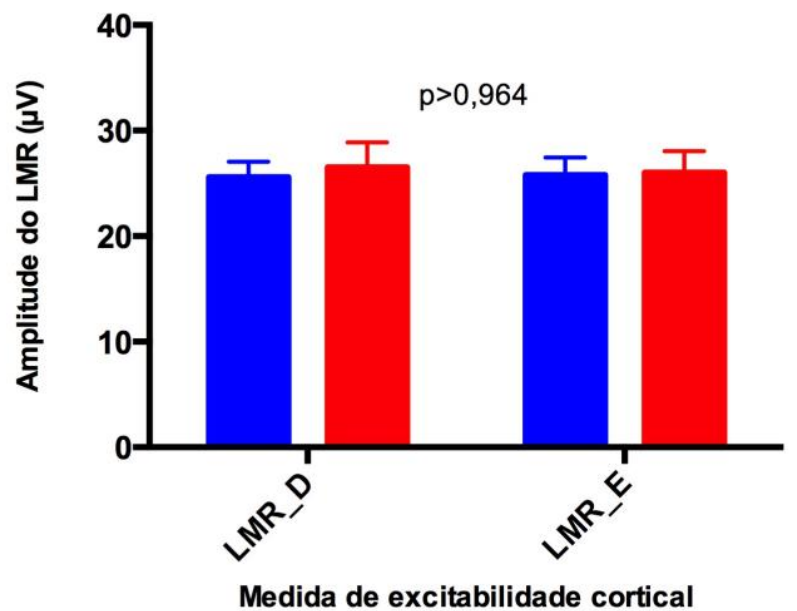

Grupo 1 (pacientes)

Grupo 2 (controles)

(*) Teste U de Mann-Whitney. As barras correspondem ao intervalo de confiança $95 \%$.

$\left({ }^{* *}\right)$ LMR=limiar motor de repouso; PEM=potencial evocado motor; IIC=inibição intracortical;

FIC=facilitação intracortical; $D=$ direito; $E=$ =esquerdo.

Figura 7 - Amplitude do limiar motor de repouso $(\mu \mathrm{V})$ dos doentes com DFA comparados aos controles saudáveis no tempo basal e teste de associação (p) $(N=57)$.

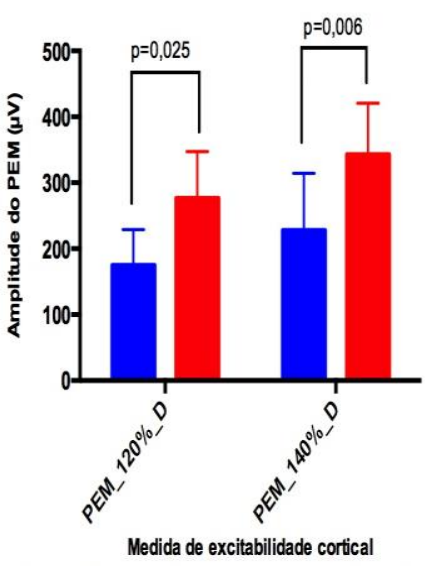

(*) Teste U de Mann-Whitney. As barras correspondem ao intervalo de confiança 95\%. $(* *)$ LMR=limiar motor de repouso; PEM=potencial evocado motor, IIC=inibição intracortical; $\mathrm{FIC}=$ facilitação intracortical; $D=$ direito; $E=$ esquerdo.

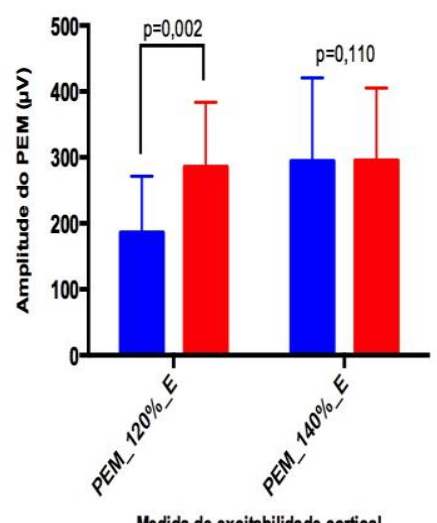

Grupo 1 (pacientes) Grupo 2 (controles)

Figura 8 - Amplitude do potencial evocado motor $(\mu \mathrm{V})$ dos doentes com DFA comparados aos controles saudáveis no tempo basal e teste de associação $(p)(N=57)$. 


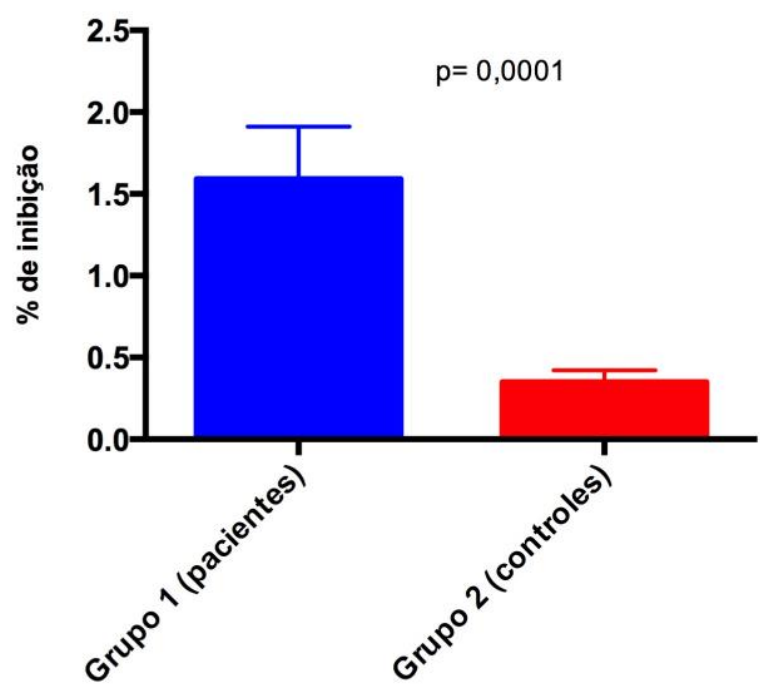

Grupo 1 (pacientes)

Grupo 2 (controles)

Medida de excitabilidade cortical

(*) Teste $U$ de Mann-Whitney. As barras correspondem ao intervalo de confiança $95 \%$.

$(\star \star) ~ L M R=l i m i a r$ motor de repouso; PEM=potencial evocado motor; IIC=inibição intracortical; $\mathrm{FIC}=$ facilitação intracortical; $D=$ direito; $E=$ esquerdo.

Figura 9 - Porcentagem de inibição intracortical dos doentes com DFA comparados aos controles saudáveis no tempo basal e teste de associação $(p)(N=57)$.

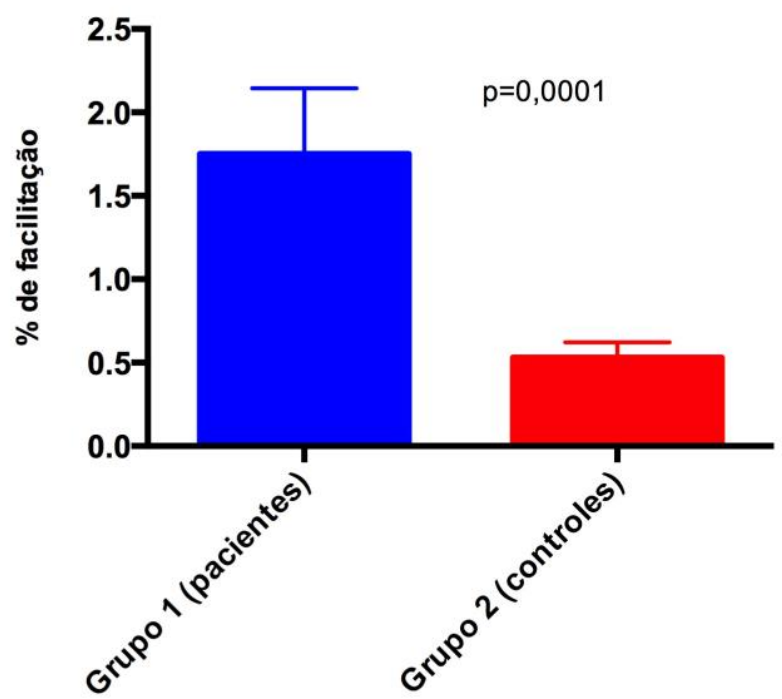

Grupo 1 (pacientes)

Grupo 2 (controles)

\section{Medida de excitabilidade cortical}

$\left.{ }^{*}\right)$ Teste $U$ de Mann-Whitney. As barras correspondem ao intervalo de confiança $95 \%$.

(**) LMR=limiar motor de repouso; PEM=potencial evocado motor; IIC=inibição intracortical;

$\mathrm{FIC}=$ facilitação intracortical; $\mathrm{D}=$ =direito; $\mathrm{E}=$ =esquerdo.

Figura 10 - Porcentagem de facilitação intracortical dos doentes com DFA comparados aos controles saudáveis no tempo basal e teste de associação (p) (N=57). 


\subsection{Avaliação comparativa ao longo do tratamento}

\subsubsection{Avaliação no tempo D7}

Pode-se observar diferença numérica, mas não estatística quanto à avaliação da dor pelo NPSI e BPI, quanto à qualidade de vida (SF-36) (Tabela 11) e quanto à excitabilidade cortical (Figuras 11, 12, 13 e 14).

Tabela 11 - Avaliação dos doentes através do NPSI, Questionário de Dor McGill, BPI e SF-36 de acordo com o grupo em médias e desvio padrão, e resultados dos testes de associação (p) no tempo D7 ( $\mathrm{N}=29)$

\begin{tabular}{llccccc}
\hline & & \multicolumn{4}{c}{ Grupos $^{*}$} \\
\cline { 2 - 5 } Características & \multicolumn{2}{c}{$\begin{array}{c}\text { Grupo 1 } \\
\text { (Ativo) }\end{array}$} & $\begin{array}{c}\text { Grupo 2 } \\
\text { (Placebo) }\end{array}$ & \\
\cline { 2 - 5 } & Categorias & $\mathbf{N}=\mathbf{1 5}$ & DP & N=14 & DP & p $^{\star *}$ \\
\hline \hline NPSI & & 40,73 & $\pm 27,40$ & 35,78 & $\pm 21,45$ & 0,949 \\
\hline McGill & Afetivo & 8,06 & $\pm 3,59$ & 8,00 & $\pm 3,90$ & 1,000 \\
& Sensitivo & 15,73 & $\pm 7,37$ & 13,64 & $\pm 7,57$ & 0,377 \\
& Total & 23,80 & $\pm 10,07$ & 21,64 & $\pm 10,87$ & 0,505 \\
\hline BPI & Dor mais fraca & 4,5 & $\pm 3,0$ & 4,1 & $\pm 1,9$ & 0,685 \\
& Média de dor & 5,9 & $\pm 2,7$ & 5,5 & $\pm 2,3$ & 0,706 \\
& Dor mais forte & 7,5 & $\pm 2,3$ & 7,3 & $\pm 1,9$ & 0,829 \\
& Dor no & 5,9 & $\pm 3,2$ & 5,0 & $\pm 2,8$ & 0,421 \\
& momento & & & & & \\
& Interferência & 29,33 & $\pm 23,50$ & 30,00 & $\pm 19,90$ & 0,780 \\
\hline SF-36 & Capacidade & 61,66 & $\pm 25,40$ & 60,00 & $\pm 24,88$ & 0,880 \\
& Funcional & & & & & \\
& Aspectos & 43,33 & $\pm 42,74$ & 26,78 & $\pm 31,72$ & 0,270 \\
& Físicos & & & & \\
& Estado Geral de & 61,86 & $\pm 11,28$ & 61,35 & $\pm 12,20$ & 0,914 \\
& saúde & & & & & \\
& Vitalidade & 48,66 & $\pm 29,18$ & 46,42 & $\pm 19,35$ & 0,747 \\
& Aspectos & 62,50 & $\pm 32,38$ & 60,71 & $\pm 25,87$ & 0,880 \\
& Sociais & & & & & \\
& Aspectos & 44,44 & $\pm 44,83$ & 33,33 & $\pm 41,34$ & 0,533 \\
& Emocionais & & & & & \\
& Saúde Mental & 55,20 & $\pm 23,03$ & 47,42 & $\pm 22,01$ & 0,331 \\
& Dor & 45,86 & $\pm 26,93$ & 48,92 & $\pm 22,87$ & 0,914 \\
\hline
\end{tabular}

**Teste U de Mann-Whitney. McGill: Questionário de dor McGill; NPSI: Inventário de Sintomas de Dor Neuropática; DN4: questionário para diagnóstico de dor neuropática; SF36: Inventário de qualidade de vida; BPI: Inventário Breve de Dor; EVA: Escala Visual Analógica; DP: Desvio Padrão. 


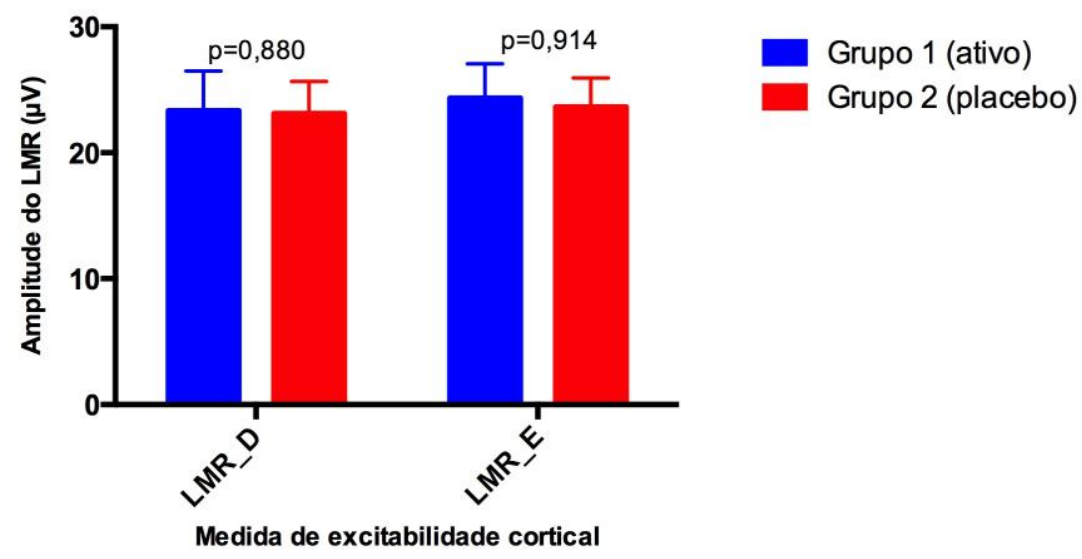

$\left(^{*}\right)$ Teste $U$ de Mann-Whitney. As barras correspondem ao intervalo de confiança $95 \%$.

$\left(^{* *}\right)$ LMR=limiar motor de repouso; PEM=potencial evocado motor; IIC=inibição intracortical; $\mathrm{FIC}=$ facilitação intracortical; $\mathrm{D}=$ direito; $E=e$ esquerdo.

Figura 11 - Média de amplitude do limiar motor de repouso $(\mu \mathrm{V})$ e intervalo de confiança dos doentes com DFA no tempo D7 e teste de associação $(p)(N=29)$.

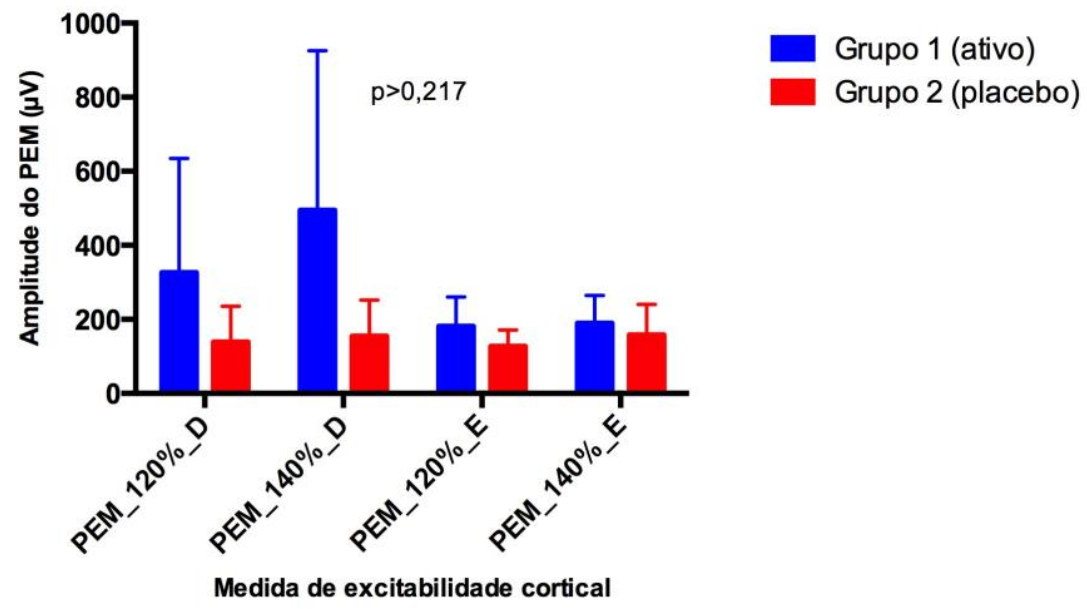

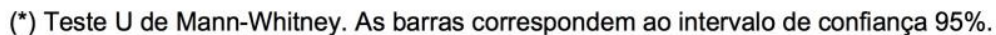

$\left({ }^{* *}\right)$ LMR=limiar motor de repouso; PEM=potencial evocado motor; IIC=inibição intracortical; FIC=facilitação intracortical; $D=$ direito; $E=$ =esquerdo.

Figura 12 - Média de amplitude do potencial evocado motor $(\mu \mathrm{V})$ e intervalo de confiança dos doentes com DFA no tempo D7 e teste de associação $(p)(N=29)$. 


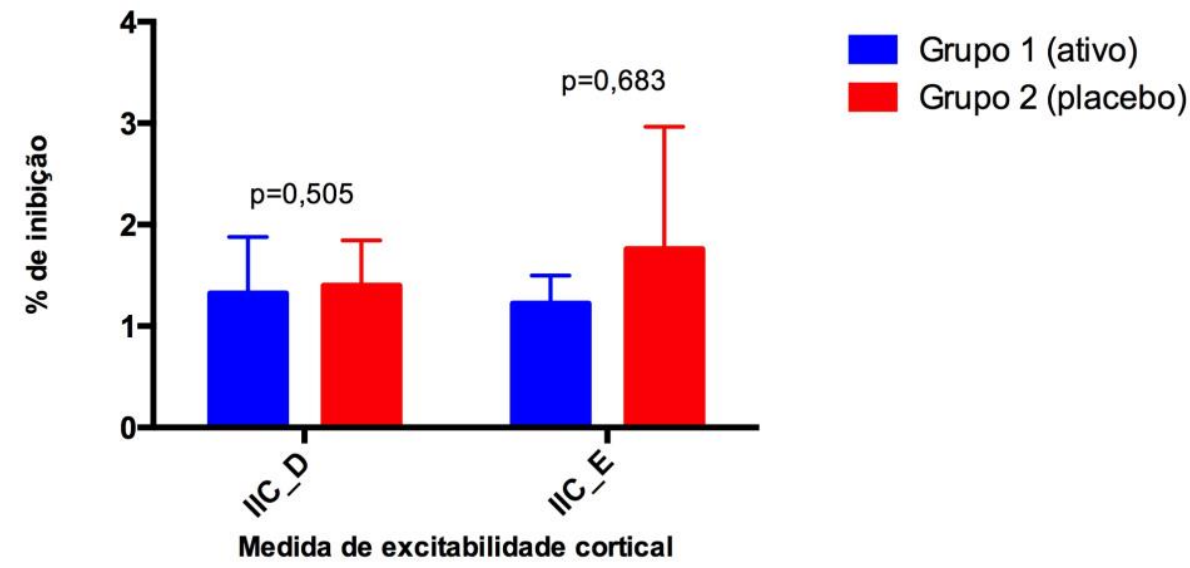

(*) Teste $U$ de Mann-Whitney. As barras correspondem ao intervalo de confiança $95 \%$.

(*) LMR=limiar motor de repouso; PEM=potencial evocado motor; IIC=inibição intracortical; $\mathrm{FIC}=$ facilitação intracortical; $\mathrm{D}=$ direito; $\mathrm{E}=$ esquerdo.

Figura 13 - Média da porcentagem de inibição intracortical e intervalo de confiança dos doentes com DFA no tempo D7 e teste de associação $(p)(N=29)$.

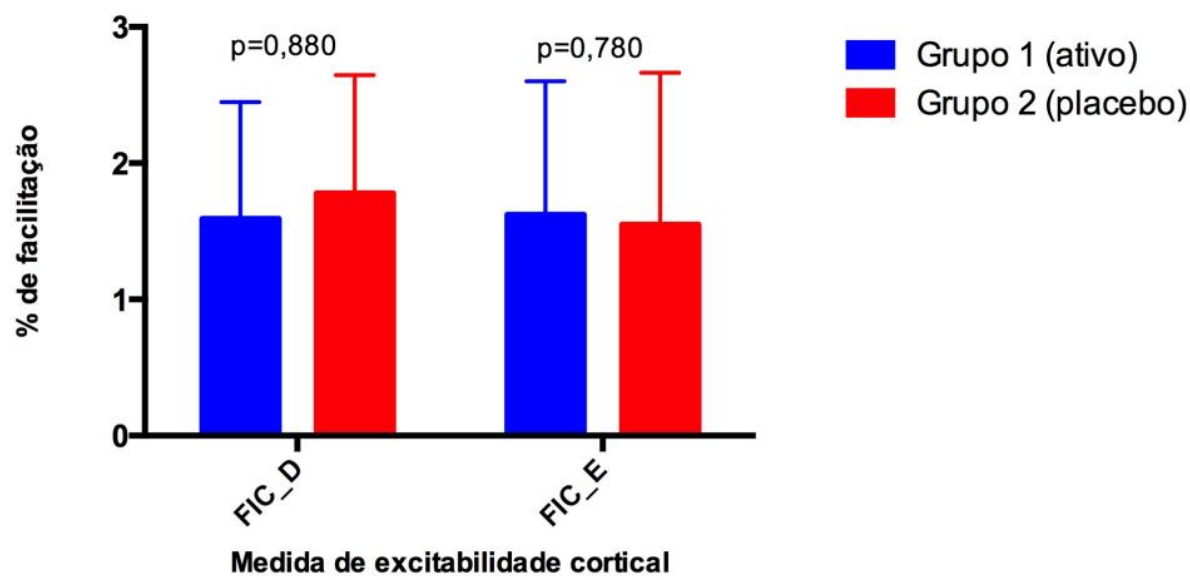

(*) Teste U de Mann-Whitney. As barras correspondem ao intervalo de confiança 95\%.

$\left({ }^{* *}\right)$ LMR=limiar motor de repouso; PEM=potencial evocado motor; IIC=inibição intracortical; $\mathrm{FIC}=$ facilitação intracortical; $\mathrm{D}=$ direito; $E=$ =squerdo.

Figura 14 - Média da porcentagem de facilitação intracortical e intervalo de confiança dos doentes com DFA no tempo D7 e teste de associação $(p)(N=29)$. 


\subsubsection{Avaliação no tempo D21}

$\mathrm{Na}$ avaliação da terceira semana (tempo D21), há diferença não estatística nas avaliações através do NPSI, questionário de dor McGill e BPI (Tabela 12). A amplitude do PEM foi maior no grupo ativo. Não houve diferença estatística na avaliação da excitabilidade cortical para a IIC e FIC entre os grupos ativo e placebo, contudo há diferença percentual de inibição e facilitação: o grupo 1 (ativo) apresentou valores mais próximos dos controles do que o grupo 2 (placebo) (Figuras 15, 16, 17 e 18).

Tabela 12 - Avaliação dos doentes através do DN4, NPSI, Questionário de Dor McGill, BPI e SF-36 de acordo com o grupo em médias e desvio padrão, e resultados dos testes de associação (p) no tempo D21 ( $N=29)$

\begin{tabular}{|c|c|c|c|c|c|c|}
\hline \multirow{4}{*}{$\begin{array}{l}\text { Características } \\
\text { NPSI }\end{array}$} & \multirow[b]{3}{*}{ Categorias } & \multicolumn{4}{|c|}{ Grupos $^{*}$} & \multirow[b]{3}{*}{$\mathbf{p}^{\star *}$} \\
\hline & & \multicolumn{2}{|c|}{ Grupo 1 (Ativo) } & \multicolumn{2}{|c|}{ Grupo 2 (Placebo) } & \\
\hline & & $\mathrm{N}=15$ & DP & $\mathrm{N}=14$ & DP & \\
\hline & & 38,00 & $\pm 26,98$ & 36,14 & $\pm 25,40$ & 0,847 \\
\hline \multirow[t]{3}{*}{ McGill } & Afetivo & 7,33 & $\pm 4,04$ & 8,07 & $\pm 4,00$ & 0,591 \\
\hline & Sensitivo & 16,40 & $\pm 7,62$ & 13,92 & $\pm 6,92$ & 0,561 \\
\hline & Total & 23,73 & $\pm 10,68$ & 22,00 & $\pm 10,04$ & 0,683 \\
\hline \multirow[t]{5}{*}{ BPI } & Dor mais fraca & 4,9 & $\pm 3,5$ & 4,1 & $\pm 2,7$ & 0,511 \\
\hline & Média de dor & 6,0 & $\pm 3,5$ & 5,5 & $\pm 2,7$ & 0,719 \\
\hline & Dor mais forte & 7,1 & $\pm 3,1$ & 7,2 & $\pm 2,8$ & 0,943 \\
\hline & $\begin{array}{l}\text { Dor no } \\
\text { momento }\end{array}$ & 6,1 & $\pm 3,7$ & 5,6 & $\pm 3,1$ & 0,705 \\
\hline & Interferência & 29,33 & $\pm 25,58$ & 29,92 & $\pm 19,44$ & 0,813 \\
\hline \multirow[t]{8}{*}{ SF-36 } & $\begin{array}{l}\text { Capacidade } \\
\text { Funcional }\end{array}$ & 62,66 & $\pm 25,20$ & 70,71 & $\pm 23,72$ & 0,400 \\
\hline & $\begin{array}{l}\text { Aspectos } \\
\text { Físicos }\end{array}$ & 36,66 & $\pm 41,04$ & 32,14 & $\pm 46,43$ & 0,683 \\
\hline & $\begin{array}{l}\text { Estado Geral de } \\
\text { saúde }\end{array}$ & 63,20 & $\pm 13,36$ & 64,35 & $\pm 12,15$ & 0,949 \\
\hline & Vitalidade & 51,66 & $\pm 26,29$ & 47,50 & $\pm 18,57$ & 0,621 \\
\hline & $\begin{array}{l}\text { Aspectos } \\
\text { Sociais }\end{array}$ & 59,16 & $\pm 36,43$ & 58,03 & $\pm 23,31$ & 0,780 \\
\hline & $\begin{array}{l}\text { Aspectos } \\
\text { Emocionais }\end{array}$ & 46,66 & $\pm 37,37$ & 40,47 & $\pm 43,71$ & 0,652 \\
\hline & Saúde Mental & 65,33 & $\pm 21,89$ & 65,14 & $\pm 18,98$ & 1,000 \\
\hline & Dor & 40,40 & $\pm 21,97$ & 41,57 & $\pm 22,20$ & 0,652 \\
\hline
\end{tabular}

**Teste U de Mann-Whitney. McGill: Questionário de dor McGill; NPSI: Inventário de Sintomas de Dor Neuropática; DN4: questionário para diagnóstico de dor neuropática; SF36: Inventário de qualidade de vida; BPI: Inventário Breve de Dor; EVA: Escala Visual Analógica; DP: Desvio Padrão. 


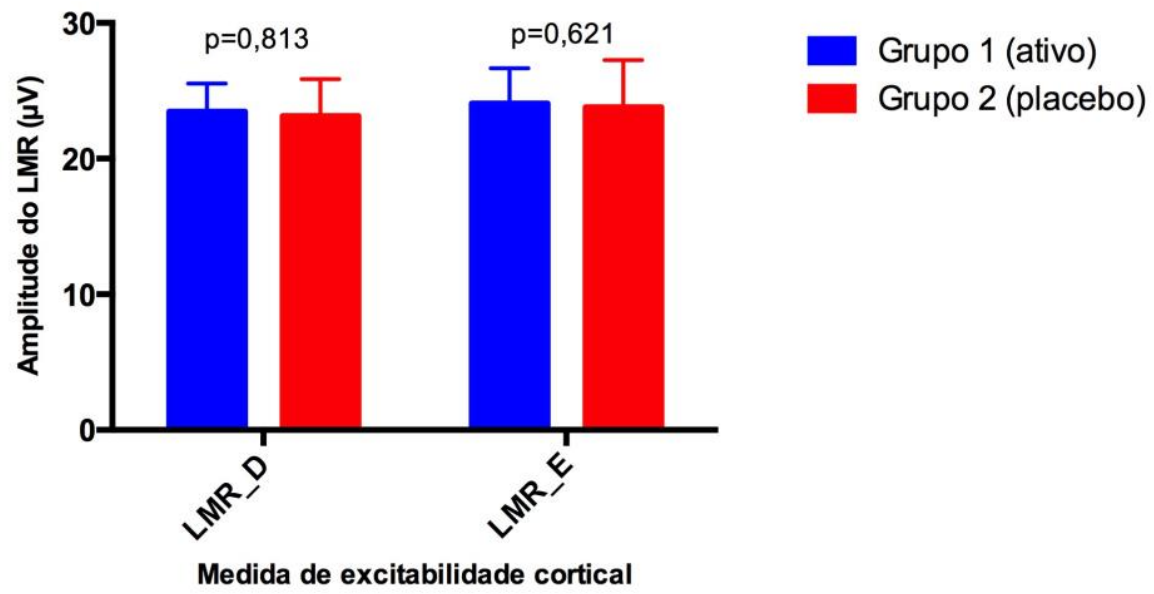

(*) Teste $U$ de Mann-Whitney. As barras correspondem ao intervalo de confiança 95\%.

${ }^{(* *}$ LMR=limiar motor de repouso; PEM=potencial evocado motor; IIC=inibição intracortical; FIC=facilitação intracortical; $D=$ direito; $E=e s q u e r d o$.

Figura 15 - Média de amplitude do limiar motor de repouso $(\mu \mathrm{V})$ e intervalo de confiança dos doentes com DFA no tempo D21 e teste de associação $(p)(N=29)$.

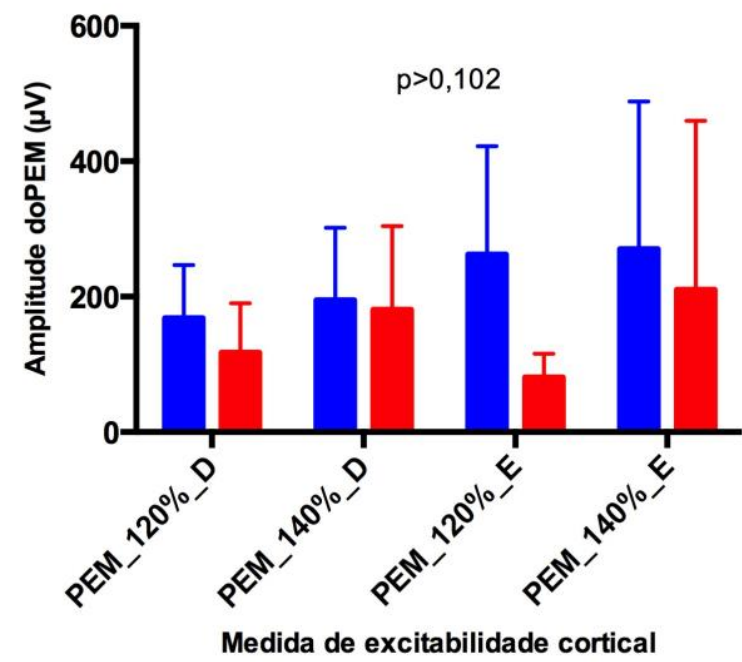

(*) Teste $U$ de Mann-Whitney. As barras correspondem ao intervalo de confiança $95 \%$.

$\left.{ }^{* *}\right)$ LMR=limiar motor de repouso; PEM=potencial evocado motor; IIC=inibição intracortical; FIC=facilitação intracortical; $D=$ direito; $E=$ esquerdo.

Figura 16 - Média de amplitude do potencial evocado motor $(\mu \mathrm{V})$ e intervalo de confiança dos doentes com DFA no tempo D21 e teste de associação $(p)(N=29)$. 


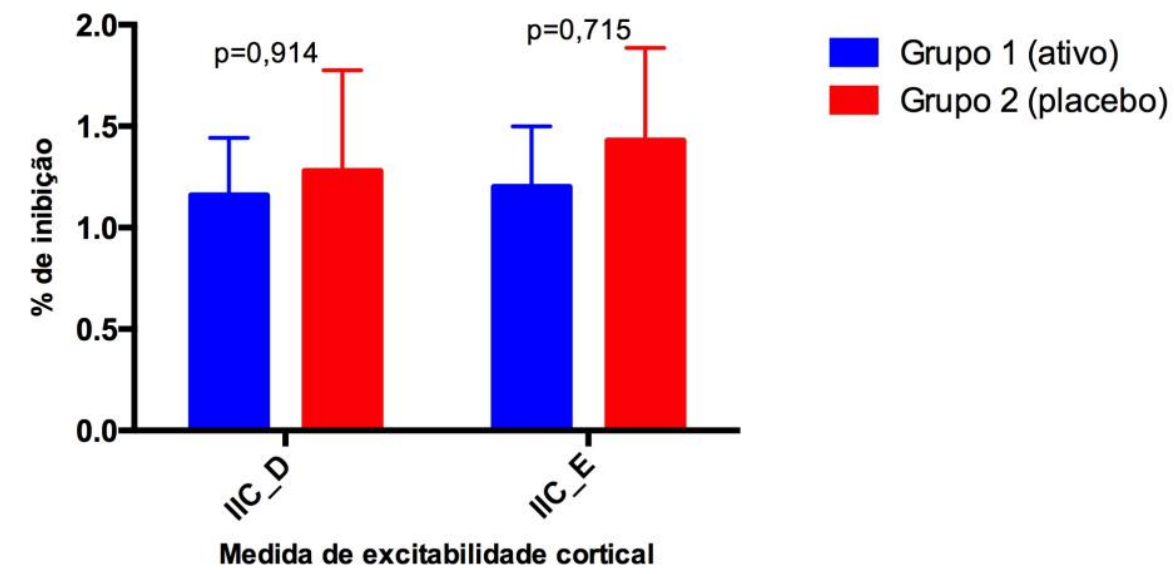

${ }^{*}$ ) Teste $U$ de Mann-Whitney. As barras correspondem ao intervalo de confiança $95 \%$.

(*) LMR=limiar motor de repouso; PEM=potencial evocado motor; IIC=inibição intracortical; $\mathrm{FIC}=$ facilitação intracortical; $\mathrm{D}=$ direito; $E=$ esquerdo.

Figura 17 - Média da porcentagem de inibição intracortical e intervalo de confiança dos doentes com DFA no tempo D21 e teste de associação $(p)(N=29)$.

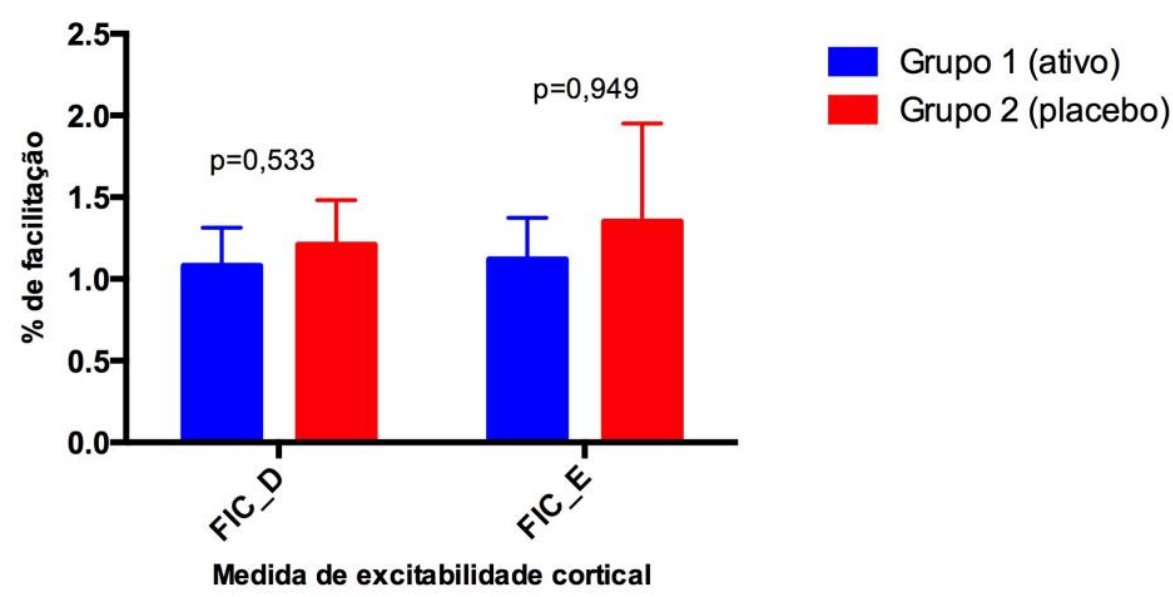

${ }^{*}$ ) Teste $U$ de Mann-Whitney. As barras correspondem ao intervalo de confiança $95 \%$.

$\left(^{* *}\right)$ LMR=limiar motor de repouso; PEM=potencial evocado motor; IIC=inibição intracortical;

$\mathrm{FIC}=$ facilitação intracortical; $\mathrm{D}=$ =direito; $E=$ =esquerdo.

Figura 18 - Média da porcentagem de facilitação intracortical e intervalo de confiança dos doentes com DFA no tempo D21 e teste de associação (p) ( $N=29)$. 


\subsubsection{Avaliação no tempo D30}

$\mathrm{Na}$ quarta semana de manutenção do tratamento, não houve diferenças quanto à intensidade média de dor (EVA; $p=0,756)$, NPSI e questionário de dor McGill. Pode ser observada diferença nos escores das categorias de capacidade funcional, aspectos físicos, estado geral de saúde e vitalidade pelo questionário SF-36 (Tabela 13). O Grupo 2 (placebo) apresentou menor potencial evocado para as amplitudes de $120 \%$ e $140 \%$ no limiar motor de repouso, sem significância estatística. Foi observada maior porcentagem de inibição intracortical no Grupo 2 (placebo) em IIC e em FIC (Figuras 19, 20, 21 e 22). 
Tabela 13 - Avaliação dos doentes através do DN4, NPSI, Questionário de Dor McGill, BPI e SF-36 de acordo com o grupo em médias e desvio padrão, e resultados dos testes de associação (p) no tempo D30 ( $N=29)$

\begin{tabular}{llccccc}
\hline & & \multicolumn{4}{c}{ Grupos $^{*}$} \\
\cline { 2 - 5 } Características & Grupo 1 (Ativo) & Grupo 2 (Placebo) & \\
\cline { 2 - 5 } & Categorias & $\mathbf{N}=\mathbf{1 5}$ & $\mathbf{D P}$ & $\mathbf{N = 1 4}$ & $\mathbf{D P}$ & $\mathbf{p}^{\star \star}$ \\
\hline \hline NPSI & & 36,60 & $\pm 28,33$ & 31,85 & $\pm 22,98$ & 0,561 \\
\hline McGill & Afetivo & 7,40 & $\pm 4,40$ & 7,14 & $\pm 3,89$ & 0,747 \\
& Sensitivo & 15,26 & $\pm 8,89$ & 11,50 & $\pm 7,14$ & 0,112 \\
& Total & 22,66 & $\pm 13,12$ & 18,64 & $\pm 10,09$ & 0,270 \\
\hline BPI & Dor mais fraca & 4,3 & $\pm 3,6$ & 3,6 & $\pm 2,7$ & 0,570 \\
& Média de dor & 5,5 & $\pm 2,9$ & 5,2 & $\pm 2,4$ & 0,756 \\
& Dor mais forte & 7,1 & $\pm 3,2$ & 7,1 & $\pm 2,6$ & 0,993 \\
& Dor no & 4,9 & $\pm 3,6$ & 4,9 & $\pm 3,0$ & 0,997 \\
& momento & & & & & \\
& Interferência & 28,33 & $\pm 26,59$ & 23,57 & $\pm 19,66$ & 0,847 \\
\hline SF-36 & Capacidade & 64,00 & $\pm 28,85$ & 65,00 & $\pm 27,59$ & 0,949 \\
& Funcional & & & & & \\
& Aspectos & 41,66 & $\pm 45,96$ & 32,14 & $\pm 43,22$ & 0,591 \\
& Físicos & & & & & \\
& Estado Geral & 53,93 & $\pm 27,16$ & 44,14 & $\pm 24,09$ & 0,354 \\
& de saúde & & & & & \\
& Vitalidade & 54,33 & $\pm 26,71$ & 48,57 & $\pm 20,79$ & 0,477 \\
& Aspectos & 57,50 & $\pm 35,91$ & 58,92 & $\pm 22,16$ & 0,983 \\
& Sociais & & & & & \\
& Aspectos & 44,44 & $\pm 43,03$ & 30,95 & $\pm 40,22$ & 0,400 \\
& Emocionais & & & & & \\
& Saúde Mental & 53,33 & $\pm 24,54$ & 52,85 & $\pm 18,24$ & 0,847 \\
& Dor & 47,20 & $\pm 24,30$ & 55,28 & $\pm 23,25$ & 0,310 \\
\hline
\end{tabular}

**Teste U de Mann-Whitney. McGill: Questionário de dor McGill; NPSI: Inventário de Sintomas de Dor Neuropática; DN4: questionário para diagnóstico de dor neuropática; SF36: Inventário de qualidade de vida; BPI: Inventário Breve de Dor; EVA: Escala Visual Analógica; DP: Desvio Padrão.

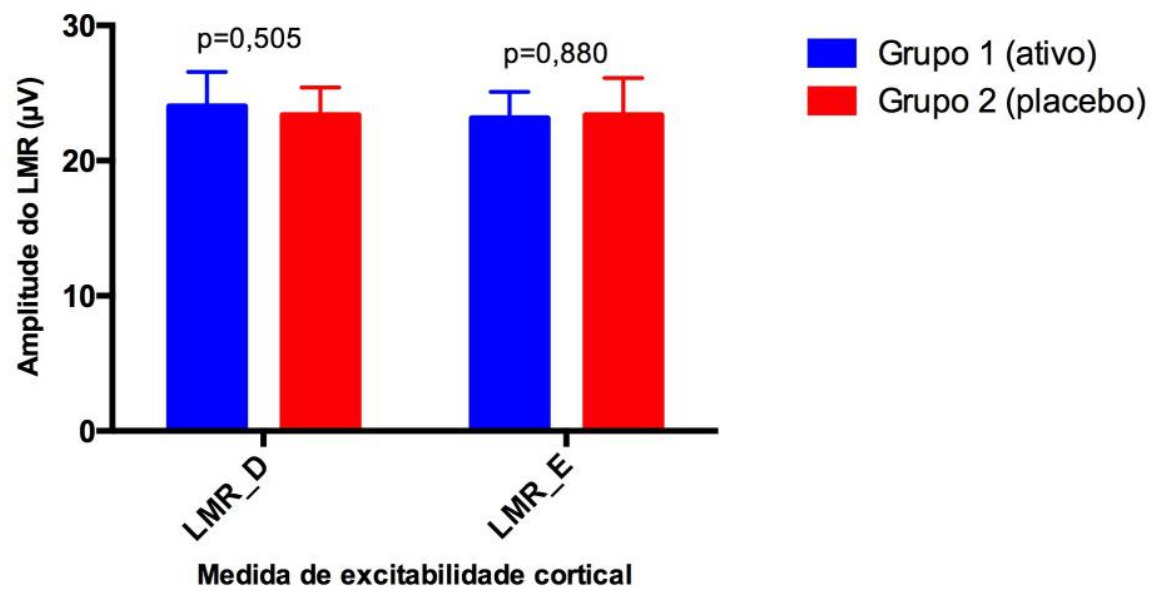

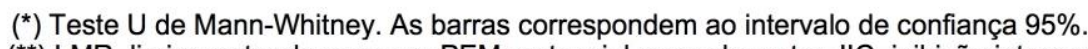

$\left({ }^{\star *}\right)$ LMR=limiar motor de repouso; PEM=potencial evocado motor; IIC=inibição intracortical;

$\mathrm{FIC}=$ facilitação intracortical; $\mathrm{D}=$ direito; $E=$ =esquerdo. 
Figura 19 - Média de amplitude do limiar motor de repouso $(\mu \mathrm{V})$ e intervalo de confiança dos doentes com DFA no tempo D30 e teste de associação $(p)(N=29)$.

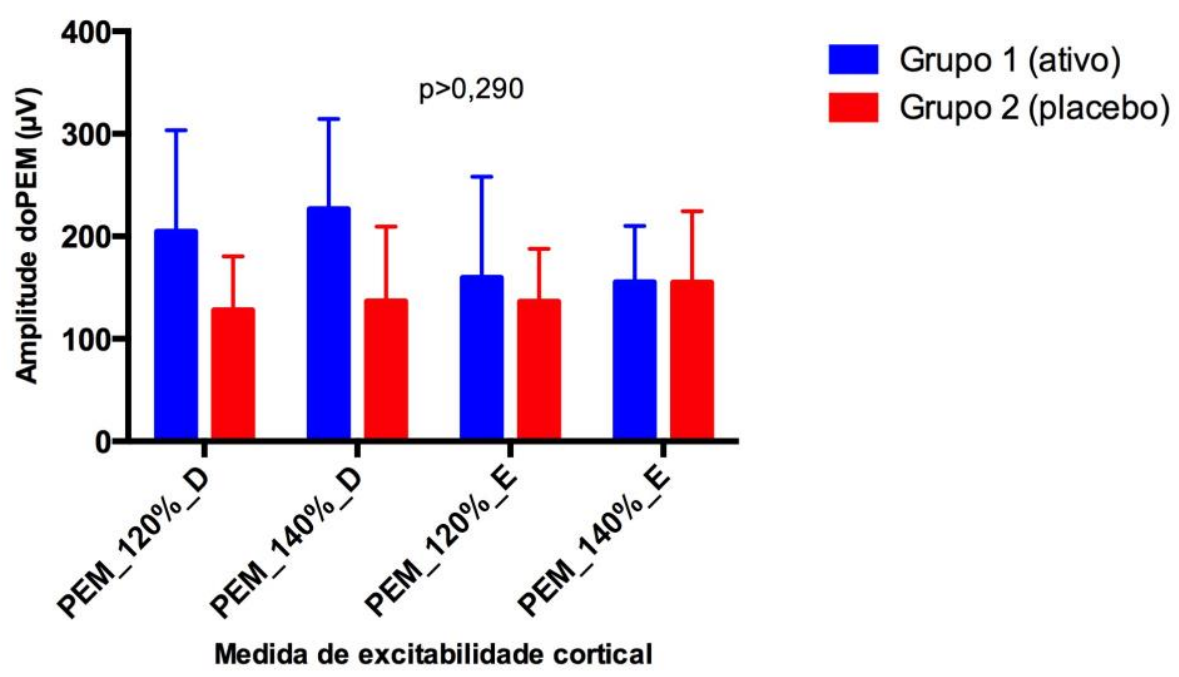

(*) Teste $U$ de Mann-Whitney. As barras correspondem ao intervalo de confiança $95 \%$.

$\left(^{* *}\right)$ LMR=limiar motor de repouso; PEM=potencial evocado motor; IIC=inibição intracortical; $\mathrm{FIC}=$ facilitação intracortical; $D=$ direito; $E=$ esquerdo.

Figura 20 - Média de amplitude do potencial evocado motor $(\mu \mathrm{V})$ e intervalo de confiança dos doentes com DFA no tempo D30 e teste de associação $(p)(N=29)$.

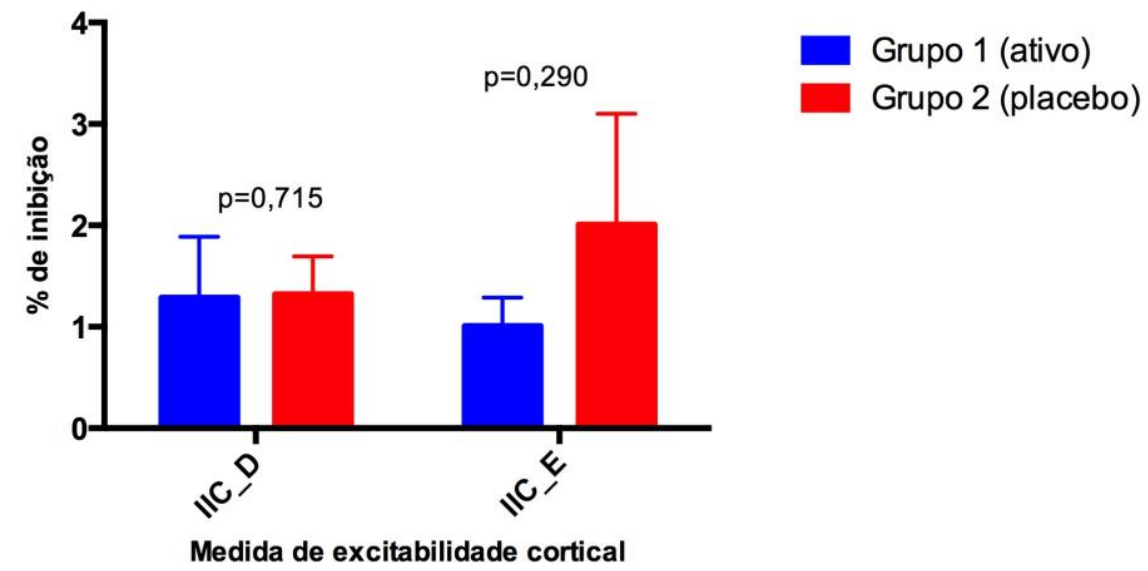

(*) Teste U de Mann-Whitney. As barras correspondem ao intervalo de confiança 95\%.

$\left(^{* *}\right)$ LMR=limiar motor de repouso; PEM=potencial evocado motor; IIC=inibição intracortical; FIC=facilitação intracortical; $D=$ direito; $E=$ esquerdo.

Figura 21 - Média da porcentagem de inibição intracortical e intervalo de confiança dos doentes com DFA no tempo D30 e teste de associação (p) (N=29). 


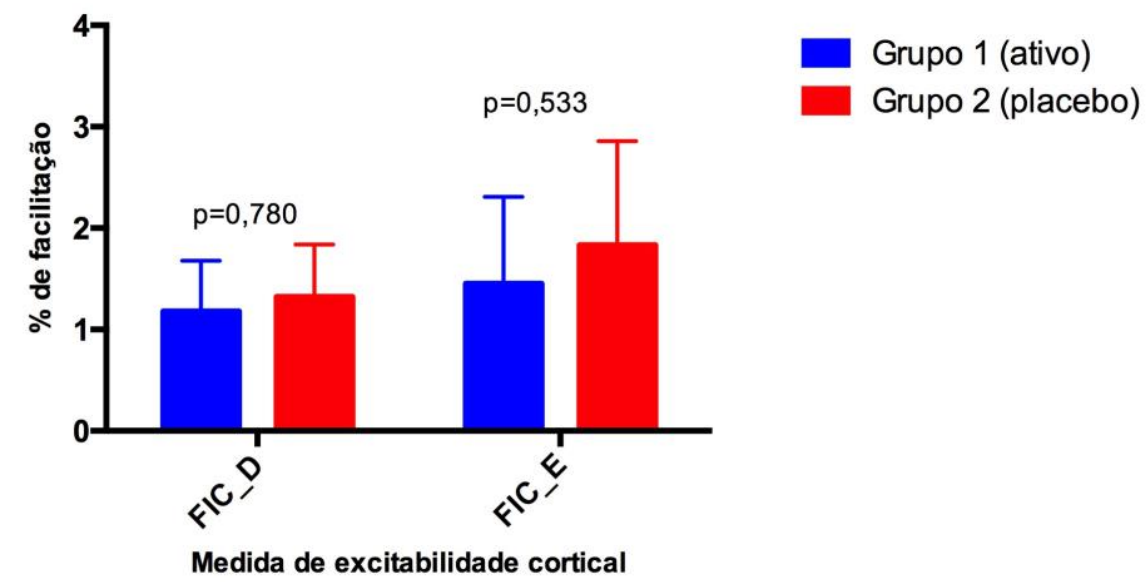

(*) Teste $U$ de Mann-Whitney. As barras correspondem ao intervalo de confiança $95 \%$.

${ }^{(*)}$ LMR=limiar motor de repouso; PEM=potencial evocado motor; IIC=inibição intracortical; $\mathrm{FIC}=$ facilitação intracortical; $D=$ direito; $E=$ =esquerdo.

Figura 22 - Média da porcentagem de facilitação intracortical e intervalo de confiança dos doentes com DFA no tempo D30 e teste de associação $(p)(N=29)$.

\subsubsection{Avaliação no tempo D60}

Na última avaliação (D60), não houve diferença estatística quanto às características da dor segundo o NPSI, o questionário de dor McGill e o BPI. O grupo 1 (Ativo) apresentou melhor desempenho no questionário de qualidade de vida SF-36, apesar de não haver significância (Tabela 14). Os resultados de excitabilidade cortical podem ser observados nas Figuras 23, 24, 25 e 26, sendo que não houve significância estatística entre os grupos, porém a amplitude do MEP foi maior no Grupo 1 (Ativo), a porcentagem de IIC foi maior no Grupo 2 (placebo) e a porcentagem de FIC foi menor no Grupo 1 (Ativo). 
Tabela 14 - Avaliação dos doentes através do DN4, NPSI, Questionário de Dor McGill, BPI e SF-36 de acordo com o grupo em médias e desvio padrão, e resultados dos testes de associação (p) no tempo D60 ( $\mathrm{N}=29)$

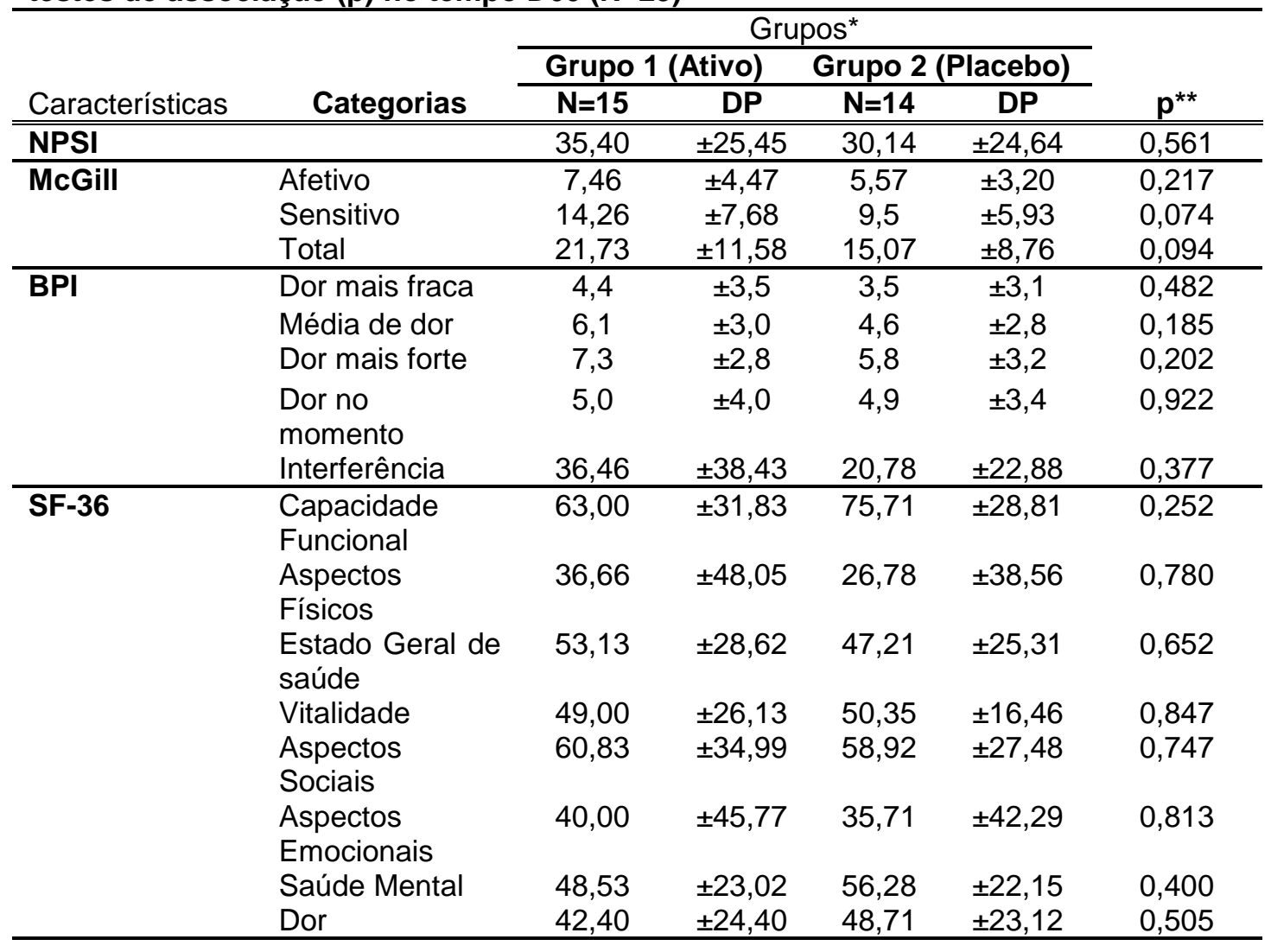

**Teste U de Mann-Whitney. McGill: Questionário de dor McGill; NPSI: Inventário de Sintomas de Dor Neuropática; DN4: questionário para diagnóstico de dor neuropática; SF36: Inventário de qualidade de vida; BPI: Inventário Breve de Dor; EVA: Escala Visual Analógica; DP: Desvio Padrão. 


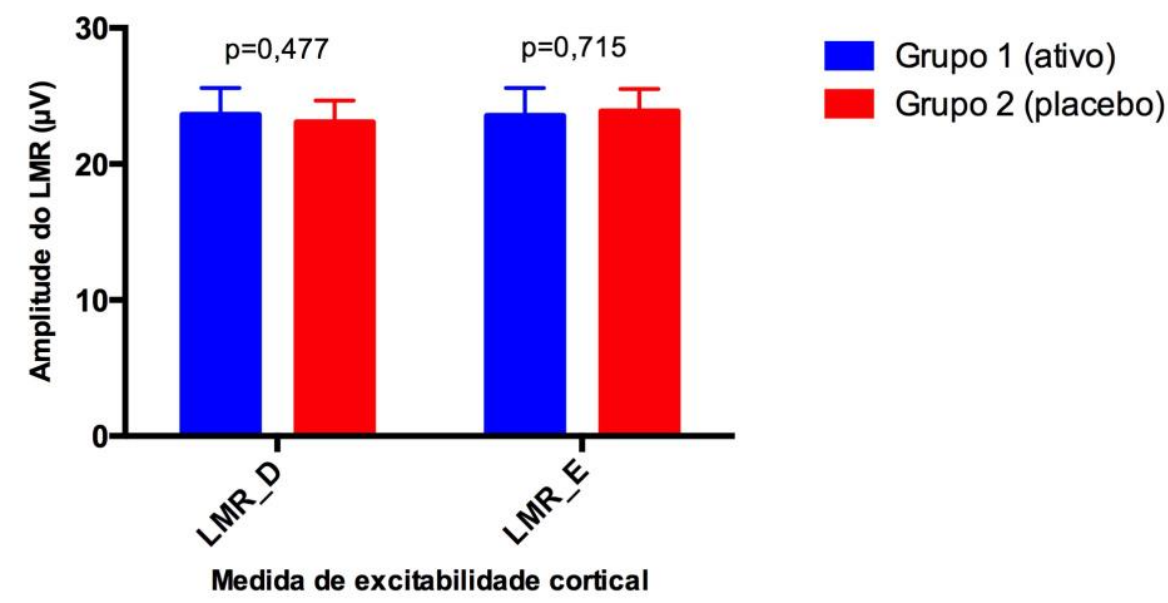

(*) Teste U de Mann-Whitney. As barras correspondem ao intervalo de confiança 95\%.

(**) LMR=limiar motor de repouso; PEM=potencial evocado motor; IIC=inibição intracortical; $F I C=$ facilitação intracortical; $D=$ direito; $E=$ esquerdo.

Figura 23 - Média de amplitude do limiar motor de repouso $(\mu \mathrm{V})$ e intervalo de confiança dos doentes com DFA no tempo D60 e teste de associação (p) (N=29).

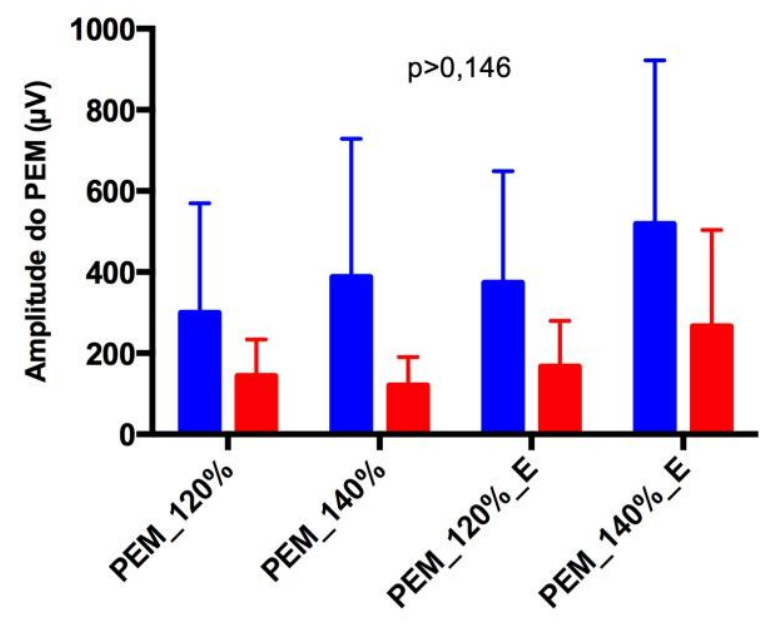

Grupo 1 (ativo)

Grupo 2 (placebo)

Medida de excitabilidade cortical

$\left(^{*}\right)$ Teste $U$ de Mann-Whitney. As barras correspondem ao intervalo de confiança $95 \%$.

${ }^{* *}$ LMR=limiar motor de repouso; PEM=potencial evocado motor; IIC=inibição intracortical; $\mathrm{FIC}=$ facilitação intracortical; $\mathrm{D}=$ direito; $E=$ esquerdo.

Figura 24 - Média de amplitude do potencial evocado motor $(\mu \mathrm{V})$ e intervalo de confiança dos doentes com DFA no tempo D60 e teste de associação (p) (N=29). 


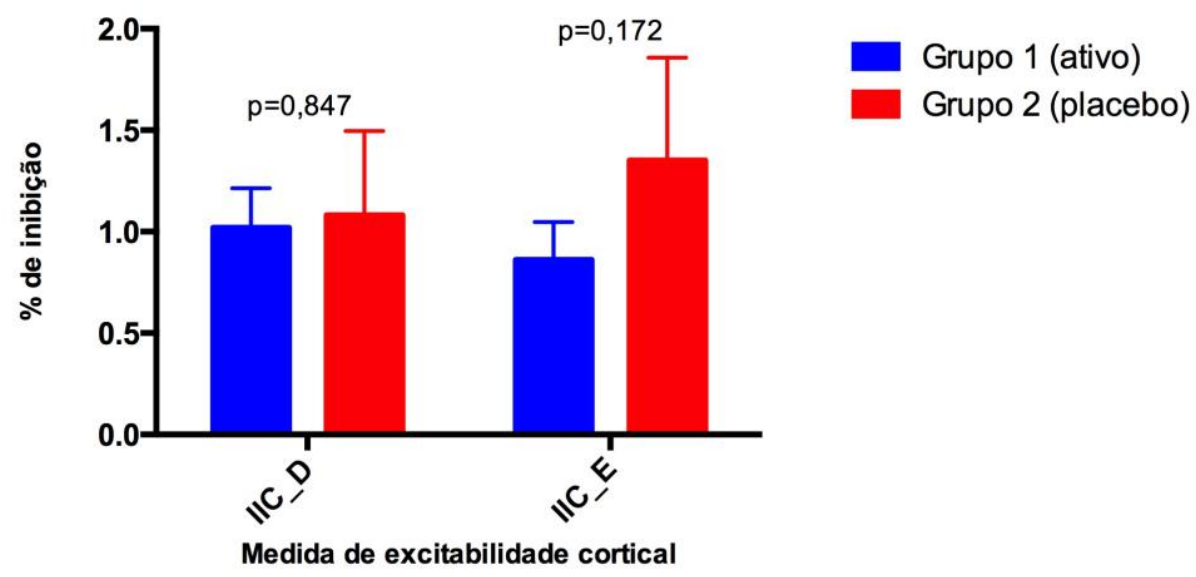

(*) Teste $U$ de Mann-Whitney. As barras correspondem ao intervalo de confiança $95 \%$.

(**) LMR=limiar motor de repouso; PEM=potencial evocado motor; IIC=inibição intracortical;

$\mathrm{FIC}=$ facilitação intracortical; $\mathrm{D}=$ direito; $\mathrm{E}=$ esquerdo.

Figura 25 - Média da porcentagem de inibição intracortical e intervalo de confiança dos doentes com DFA no tempo D60 e teste de associação (p) (N=29).
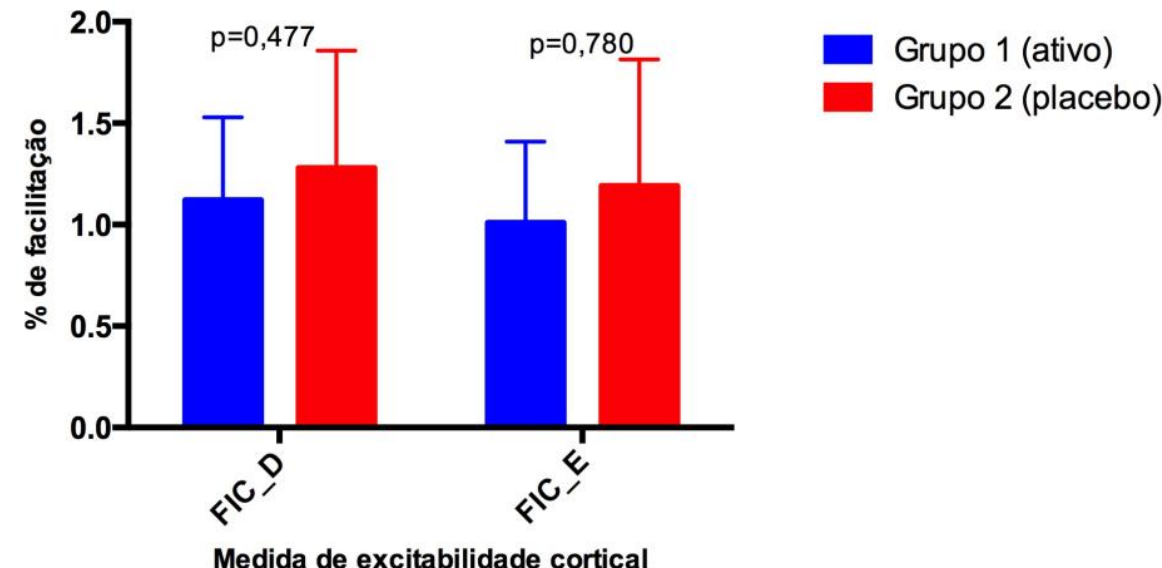

Medida de excitabilidade cortical

(*) Teste $U$ de Mann-Whitney. As barras correspondem ao intervalo de confiança 95\%.

(**) LMR=limiar motor de repouso; PEM=potencial evocado motor; IIC=inibição intracortical; $\mathrm{FIC}=$ facilitação intracortical; $D=$ direito; $E=$ =esquerdo.

Figura 26 - Média da porcentagem de facilitação intracortical e intervalo de confiança dos doentes com DFA no tempo D60 e teste de associação (p) $(\mathrm{N}=29)$.

\subsection{Comparação entre os tempos de tratamento e as avaliações}

A análise de comparação entre os tempos de avaliação apresentou melhora no resultado do questionário $\mathrm{DN}-4$ na avaliação $\mathrm{D} 21$, quando 
comparada à $D 7(P=0,048)$ (Figura 27). Não houve diferença com relação ao NPSI (Figura 28).

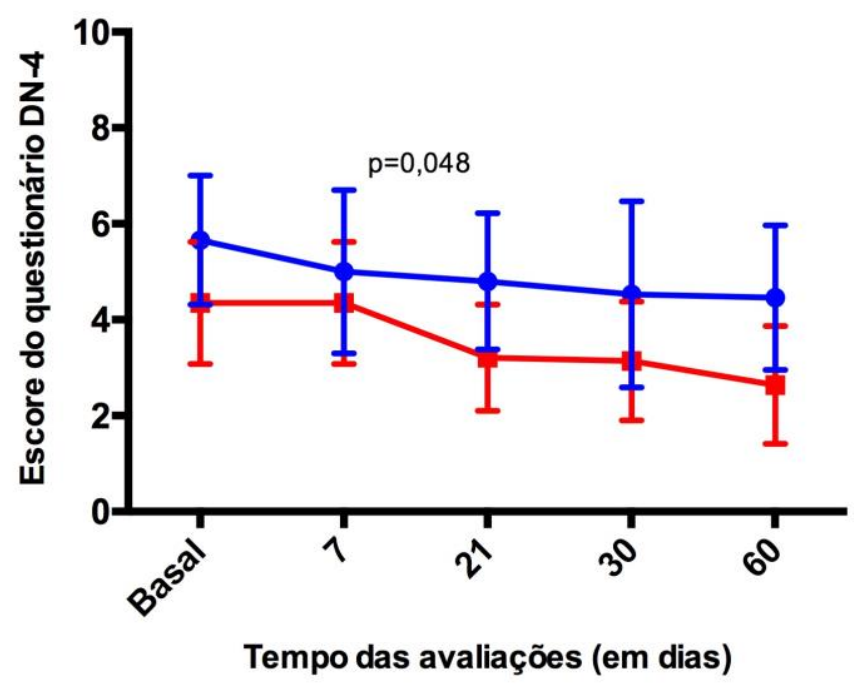

- Grupo 1 (ativo)

$\rightarrow$ Grupo 2 (placebo)

(*) Teste ANOVA de medidas repetidas, PostHoc: teste de Bonferroni. As barras correspondem ao intervalo de confiança $95 \%$.

$\left.{ }^{(* *}\right)$ DN4= Questionário de Dor Neuropática 4.

Figura 27 - Escore do questionário DN-4 dos doentes com DFA de acordo com o grupo e com o período de avaliação e intervalo de confiança, e resultados dos testes de associação (p) $(\mathrm{N}=29)$.

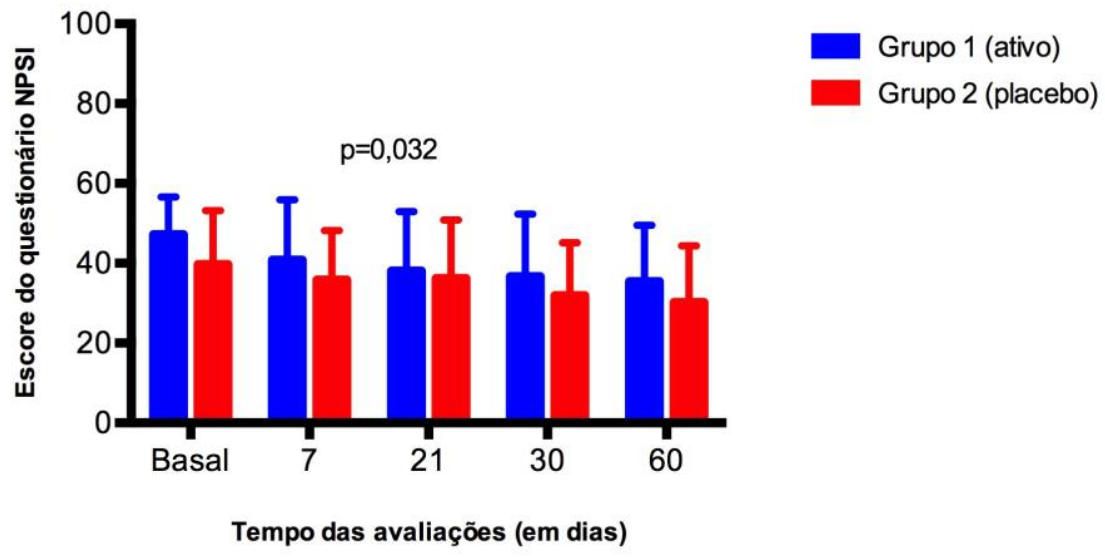

(*) Teste ANOVA de medidas repetidas, PostHoc: teste de Bonferroni. As barras correspondem ao intervalo de confiança $95 \%$.

$\left.{ }^{* *}\right) \mathrm{NPSI}=$ Inventánio de sintomas de dor neuropática.

Figura 28 - Escore do questionário NPSI dos doentes com DFA de acordo com o grupo e com o período de avaliação e intervalo de confiança, e resultados dos testes de associação (p) $(\mathrm{N}=29)$. 
Houve efeito de tempo entre as avaliações basal e D7 da subescala do BPI-interferência ( $p=0,013)$ (Figura 29), na subescala afetiva de McGill e também entre o basal e D7 ( $p=0,031)$ (Figura 30). Foi apresentada presença de tempo entre as avaliações D21 e D30 da subescala sensitiva de McGill $(p=0,058)$ (Figura 31). O escore do total do questionário McGill apresentou efeito de tempo entre as avaliações D21 e D30 ( $p=0,037)$ (Figura 32).

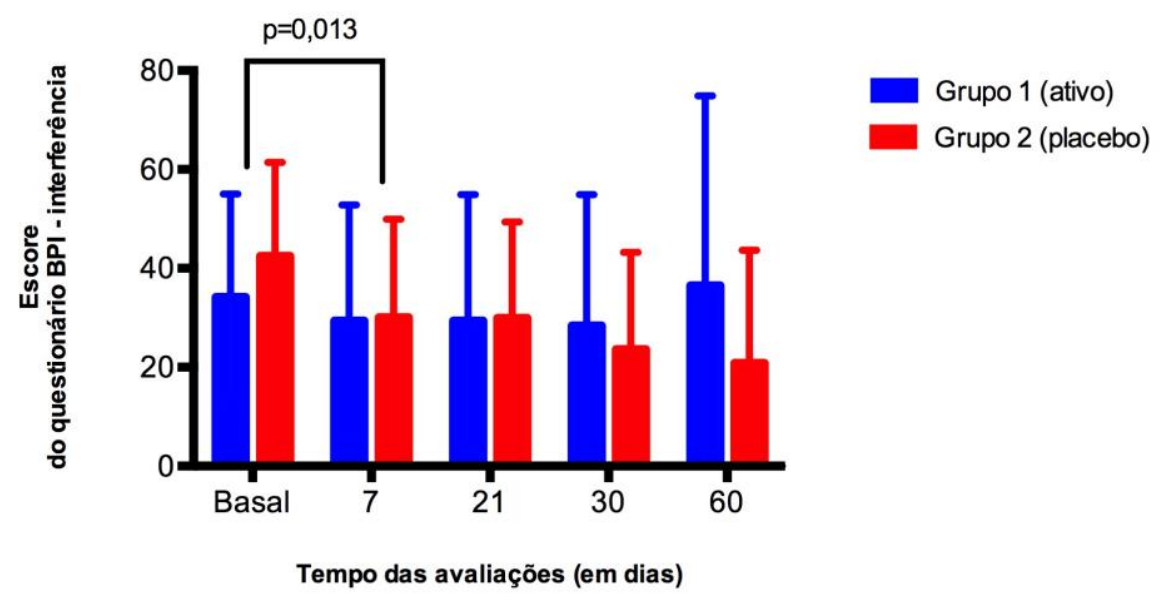

(*) Teste ANOVA de medidas repetidas, PostHoc: teste de Bonferroni. As barras correspondem ao intervalo de confiança $95 \%$.

$\left.{ }^{\star * \star}\right) \mathrm{BPI}=$ Inventário Breve de Dor.

Figura 29 - Escore da subescala interferência do questionário BPI dos doentes com DFA de acordo com o grupo e com o período de avaliação e intervalo de confiança, e resultados dos testes de associação $(p)(N=29)$. 


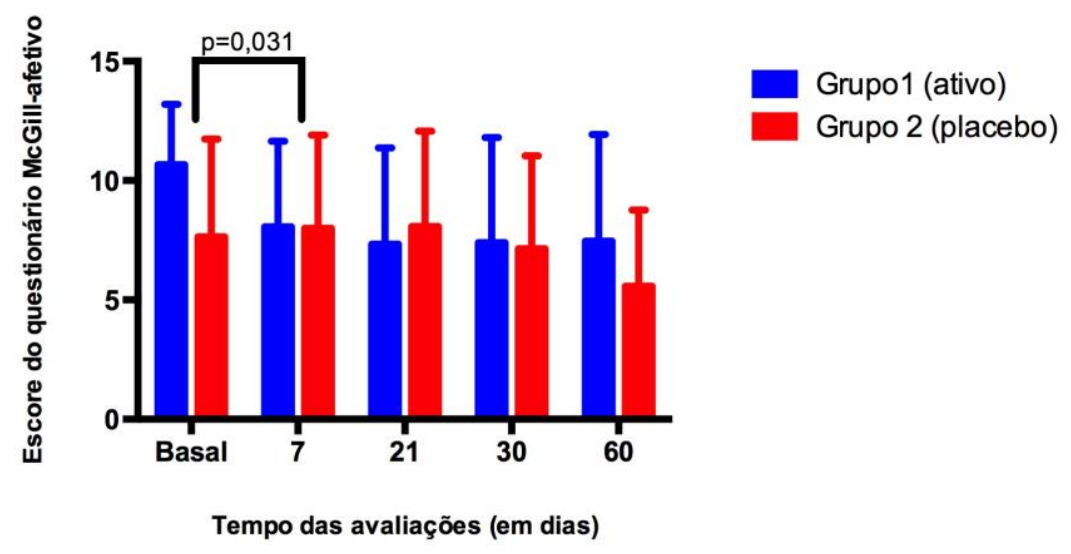

${ }^{*}$ ( ) Teste ANOVA de medidas repetidas, PostHoc: teste de Bonferroni. As barras correspondem ao intervalo de confiança $95 \%$.

$\left({ }^{*}\right)$ McGill=questionário breve de dor McGill.

Figura 30 - Escore da subescala (afetivo) do questionário breve McGill dos doentes com DFA de acordo com o grupo e com o período de avaliação e intervalo de confiança, e resultados dos testes de associação $(p)(N=29)$.

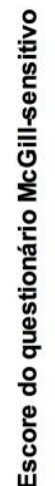

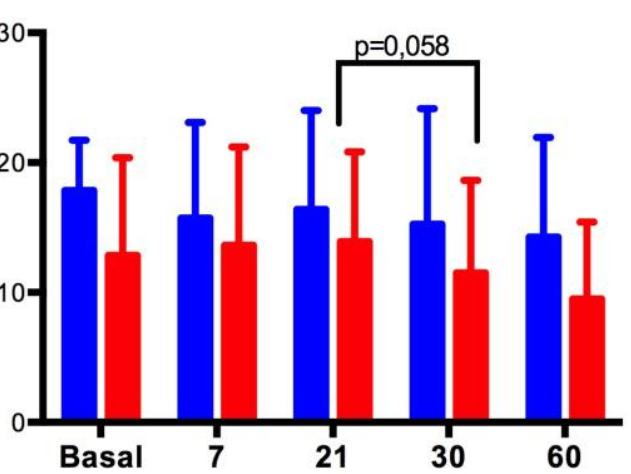

Grupo 1 (ativo)

Grupo 2 (placebo)

Tempo das avaliações (em dias)

${ }^{*}$ ) Teste ANOVA de medidas repetidas, PostHoc: teste de Bonferroni. As barras correspondem ao intervalo de confiança $95 \%$.

$\left.{ }^{* *}\right)$ McGill=questionário breve de dor McGill.

Figura 31 - Escore da subescala (sensitivo) do questionário breve McGill dos doentes com DFA de acordo com o grupo e com o período de avaliação e intervalo de confiança, e resultados dos testes de associação $(p)(N=29)$. 


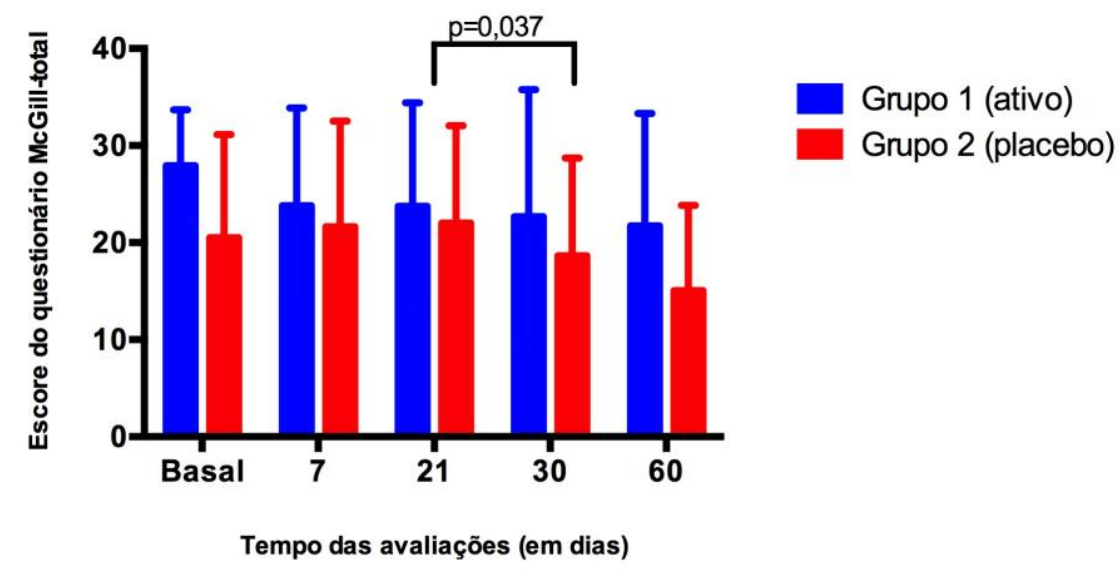

(*) Teste ANOVA de medidas repetidas, PostHoc: teste de Bonferroni. As barras correspondem ao intervalo de confiança $95 \%$.

(**) McGill=questionário breve de dor McGill.

Figura 32 - Escore total do questionário breve McGill dos doentes com DFA de acordo com o grupo e com o período de avaliação e intervalo de confiança, e resultados dos testes de associação (p) (N=29).

Pode-se observar na Figura 33 o domínio Aspecto Físico do SF-36, e na Figura 34, o domínio Aspecto Emocional, ambos sem diferença estatística entre os tempos de avaliação. A Figura 35 apresenta o domínio Aspectos Emocionais sem resultado significativo $(p=0,058)$, o domínio Capacidade Funcional (Figura 36) não apresentou resultados estatisticamente significante $(p=0,166)$. 


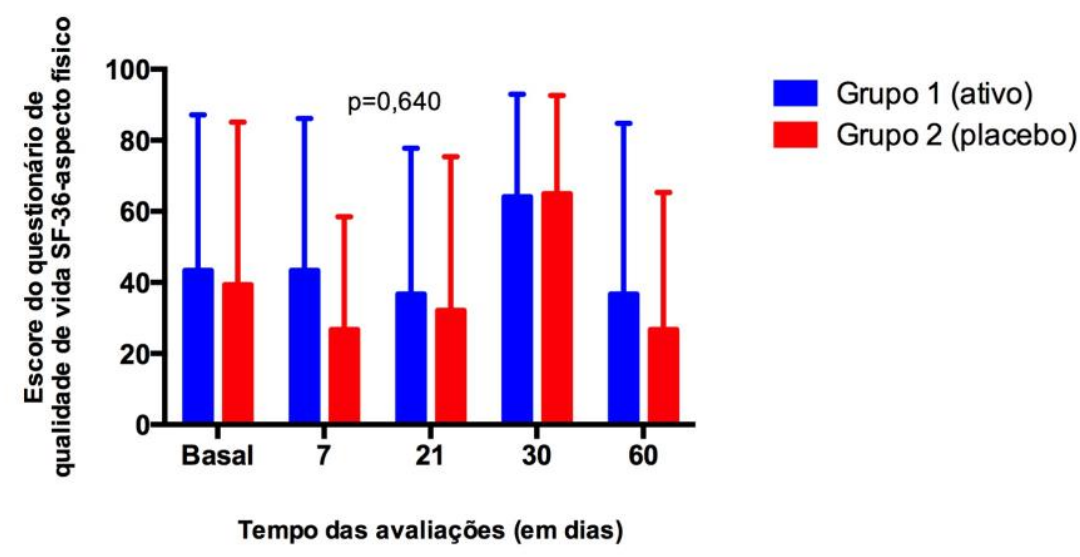

${ }^{\star}$ )Teste ANOVA de medidas repetidas, PostHoc: teste de Bonferroni. As barras comrespondem ao intervalo de confiança $95 \%$.

$\left.{ }^{* \star}\right)$ SF-36= questionánio de qualidade de vida Medical Outcomes Study 36- Item Short-Form Health Survey.

Figura 33 - Escore do questionário de qualidade de vida SF-36 domínio aspecto físico, dos doentes com DFA e intervalo de confiança, e resultados dos testes de associação (p)

$(\mathrm{N}=29)$.
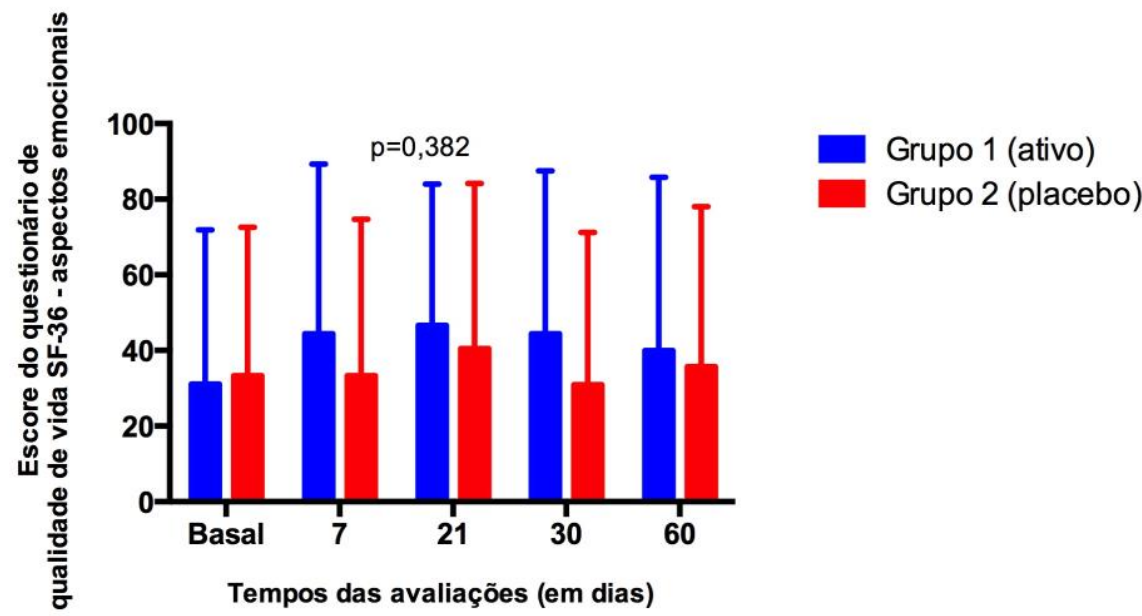

(*) Teste ANOVA de medidas repetidas, PostHoc: teste de Bonferroni. As barras correspondem ao intervalo de confiança $95 \%$.

${ }^{* *}$ SF-36= questionário de qualidade de vida Medical Outcomes Study 36- Item Short-Form Health Survey.

Figura 34 - Escore do questionário de qualidade de vida SF-36 domínio aspectos emocionais, dos doentes com DFA e intervalo de confiança, e resultados dos testes de associação (p) $(\mathrm{N}=29)$. 


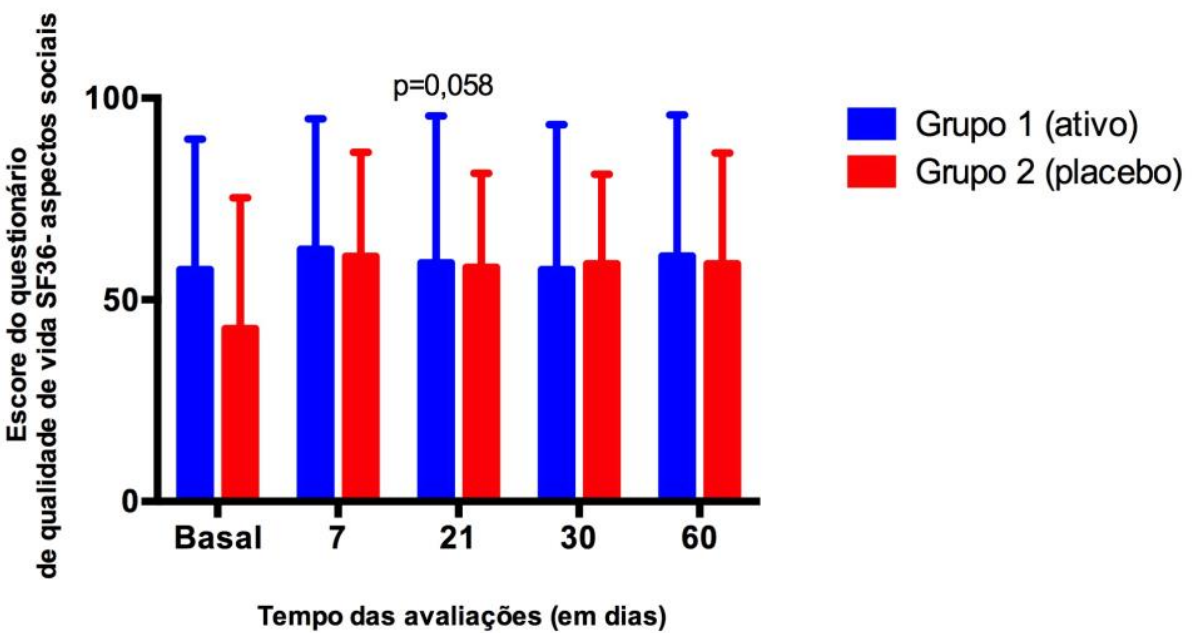

(*) Teste ANOVA de medidas repetidas, PostHoc: teste de Bonferroni. As barras correspondem ao intervalo de confiança $95 \%$.

$\left.{ }^{* \star}\right)$ SF-36= questionário de qualidade de vida Medical Outcomes Study 36- Item Short-Form Health Survey.

Figura 35 - Escore do questionário de qualidade de vida SF-36 domínio aspectos sociais, dos doentes com DFA e intervalo de confiança, e resultados dos testes de associação $(p)$ $(\mathrm{N}=29)$.

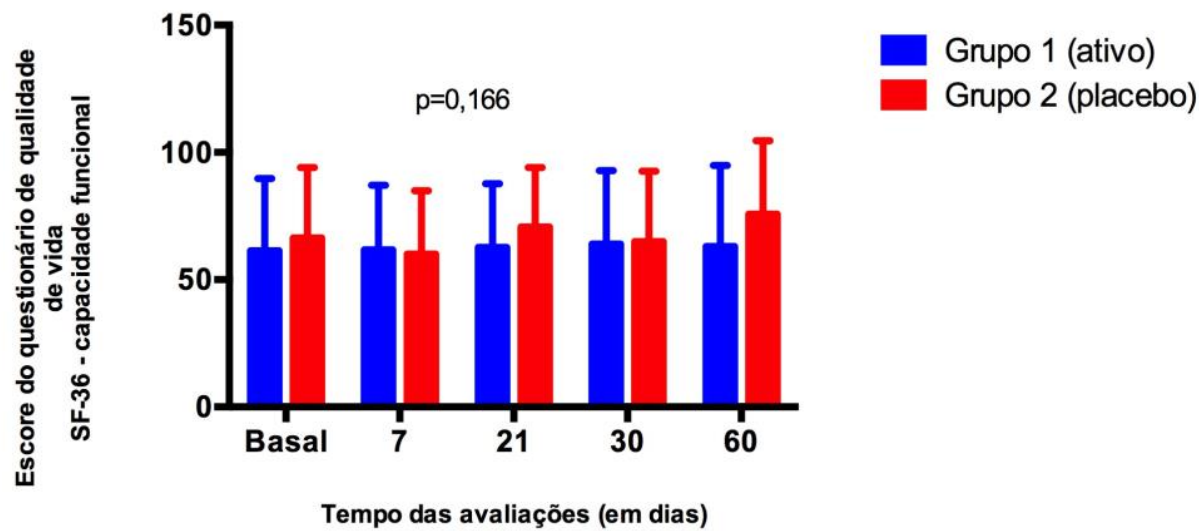

$\left(^{\star}\right)$ Teste Anova de medidas repetidas, PostHoc: teste de Bonferroni. As barras correspondem ao intervalo de confiança $95 \%$.

$\left.{ }^{* \star}\right)$ SF-36= questionário de qualidade de vida Medical Outcomes Study 36- Item Short-Form Health Survey.

Figura 36 - Escore do questionário de qualidade de vida SF-36 domínio capacidade funcional, dos doentes com DFA e intervalo de confiança, e resultados dos testes de associação $(p)(\mathrm{N}=29)$.

Houve interação entre grupos para o domínio Dor do SF-36 $(p=0,015)$ (Figura 37). Houve efeito de tempo entre as avaliações D21 e D30 para o 
domínio Estado Geral de Saúde, o grupo 2 (placebo) apresentou maior escore $(p=0,006)$ (Figura 38), a (Figura 39) apresentou interação entre os grupos para a o domínio Saúde Mental do SF-36 (p=0,0001) (Figura 39), o domínio Vitalidade do SF-36 não apresentou diferença estatisticamente significante $(p=0,525)$ (Figura 40).

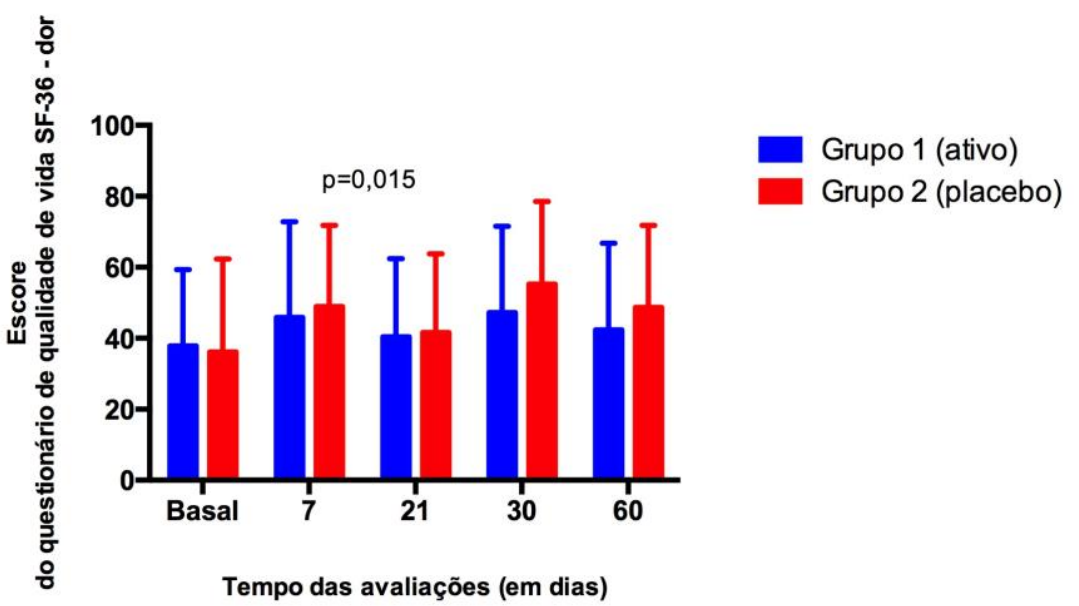

${ }^{*}$ ) Teste ANOVA de medidas repetidas, PostHoc: teste de Bonferroni. As barras correspondem ao intervalo de confiança $95 \%$

$\left.{ }^{* *}\right)$ SF-36= questionário de qualidade de vida Medical Outcomes Study 36- Item Short-Form Health Survey.

Figura 37 - Escore do questionário de qualidade de vida SF-36 domínio dor, dos doentes com DFA e intervalo de confiança, e resultados dos testes de associação $(p)(N=29)$. 


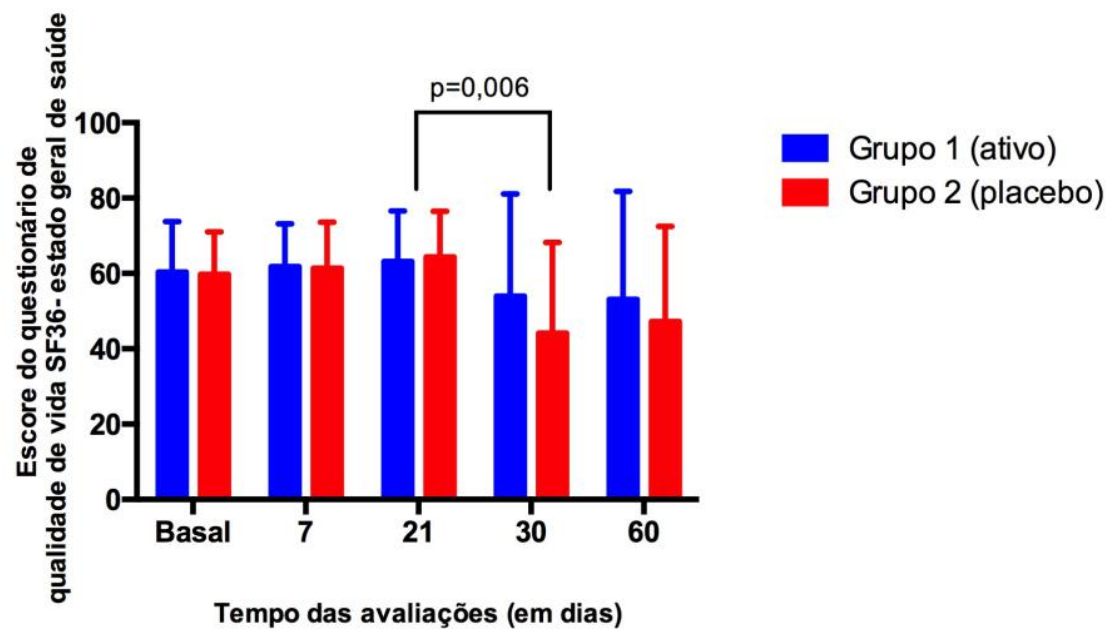

$\left({ }^{*}\right)$ Teste ANOVA de medidas repetidas, PostHoc: teste de Bonferroni. As barras correspondem ao intervalo de confiança $95 \%$.

$\left.{ }^{* *}\right)$ SF-36= questionário de qualidade de vida Medical Outcomes Study 36- Item Short-Form Health Survey.

Figura 38 - Escore do questionário de qualidade de vida SF-36 domínio estado geral de saúde, dos doentes com DFA e intervalo de confiança, e resultados dos testes de associação (p) (N=29).

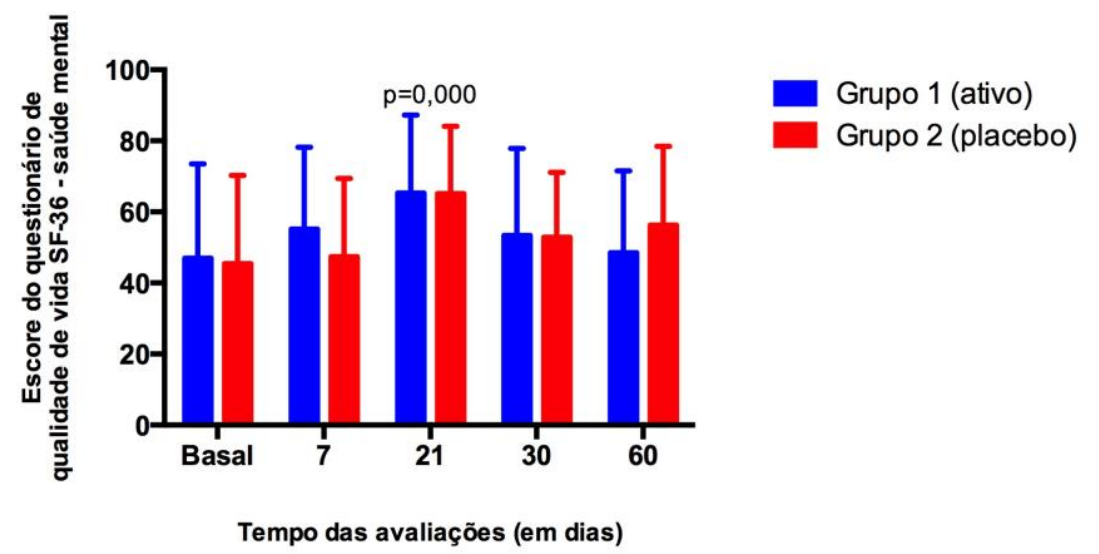

${ }^{*}$ ) Tempo ANOVA de medidas repetidas, PostHoc: teste de Bonferroni. As barras correspondem ao intervalo de confiança $95 \%$.

$\left({ }^{\star *}\right)$ SF-36= questionário de qualidade de vida Medical Outcomes Study 36-Item Short-Form Health Survey.

Figura 39 - Escore do questionário de qualidade de vida SF-36 domínio saúde mental aspecto físico, dos doentes com DFA e intervalo de confiança, e resultados dos testes de associação $(p)(N=29)$. 


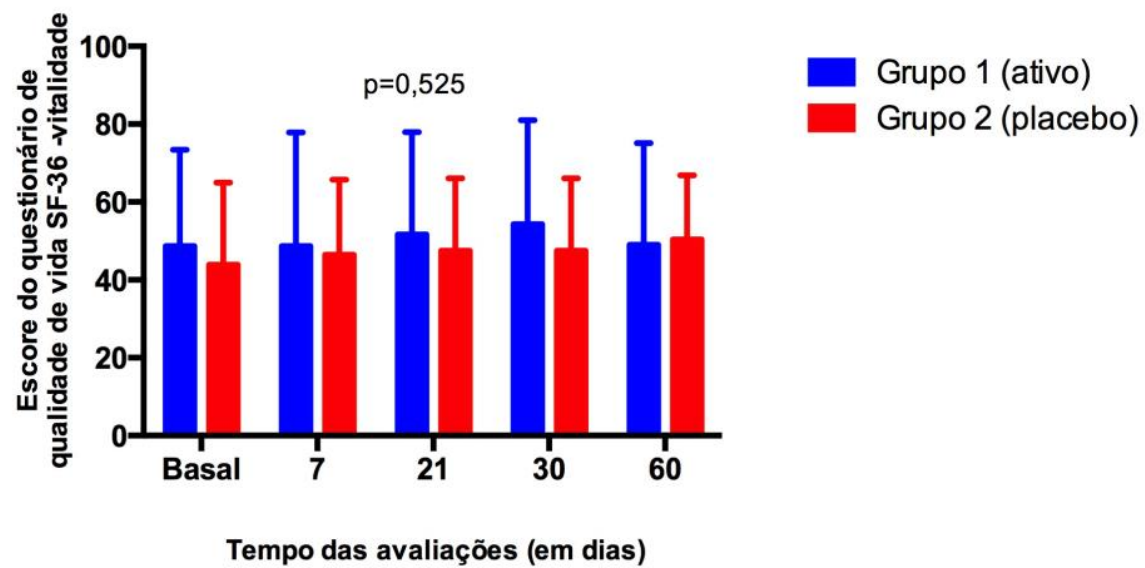

$\left.{ }^{*}\right)$ Teste ANOVA de medidas repetidas, PostHoc: teste de Bonferroni. As barras correspondem ao intervalo de confiança $95 \%$.

$\left.{ }^{(*}\right)$ SF-36= questionário de qualidade de vida Medical Outcomes Study 36- Item Short-Form Health Survey.

Figura 40 - Escore do questionário de qualidade de vida SF-36 domínio vitalidade, dos doentes com DFA e intervalo de confiança, e resultados dos testes de associação $(p)$ $(\mathrm{N}=29)$.

A média de dor mais fraca e a média de dor no momento da avaliação não apresentaram diferença estatística (Figuras 41 e 42). Houve redução na média da intensidade de dor pelo BPI e na média de dor mais intensa pela EVA em ambos os Grupos (Ativo e Placebo) ( $p=0,029$ e $p=0,027$, respectivamente) (Figuras 43 e 44). 


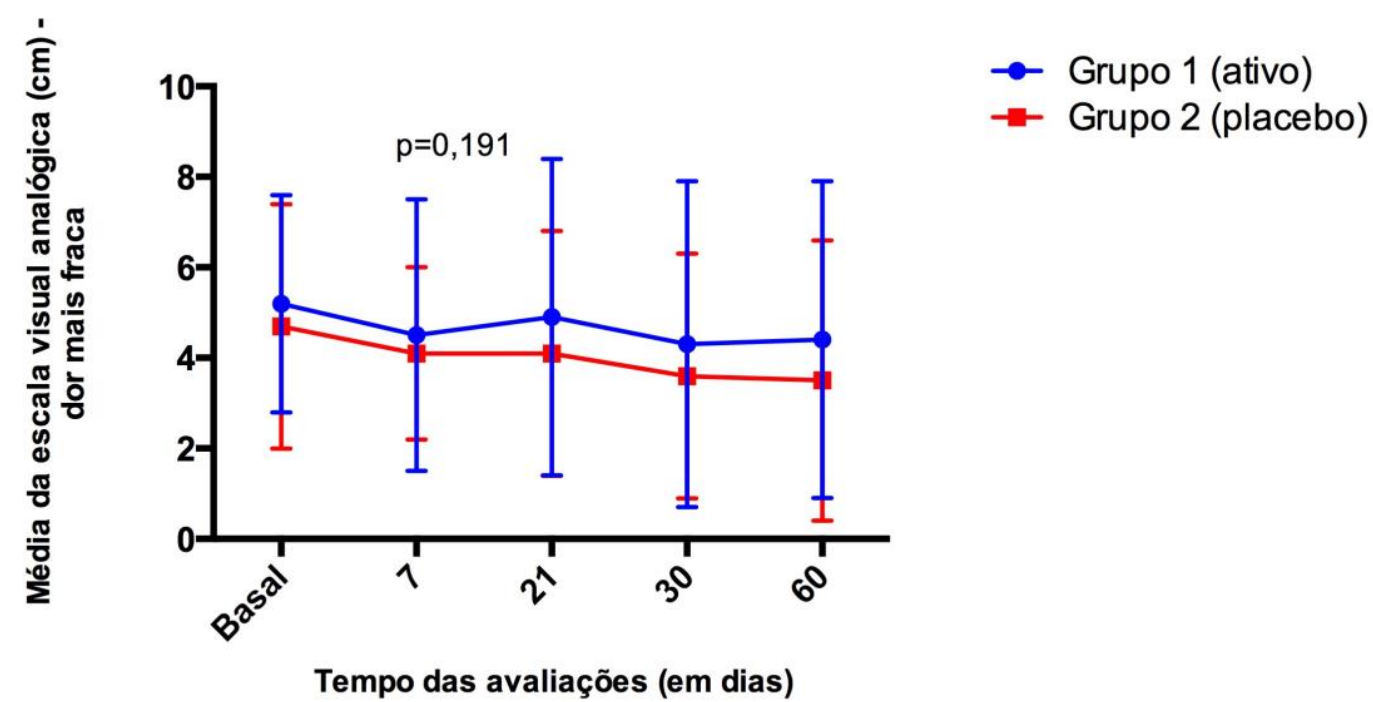

$\left(^{*}\right)$ Teste ANOVA de medidas repetidas, PostHoc: teste de Bonferroni. As barras correspondem ao intervalo de confiança $95 \%$.

$\left.{ }^{(*}\right) \mathrm{BPI}=$ Inventário Breve de Dor - subescala média de dor.

Figura 41 - Escore da subescala dor mais fraca nas últimas 24 horas do questionário BPI dos doentes com DFA e intervalo de confiança, e resultado dos testes de associação ( $p)$ $(\mathrm{N}=29)$.

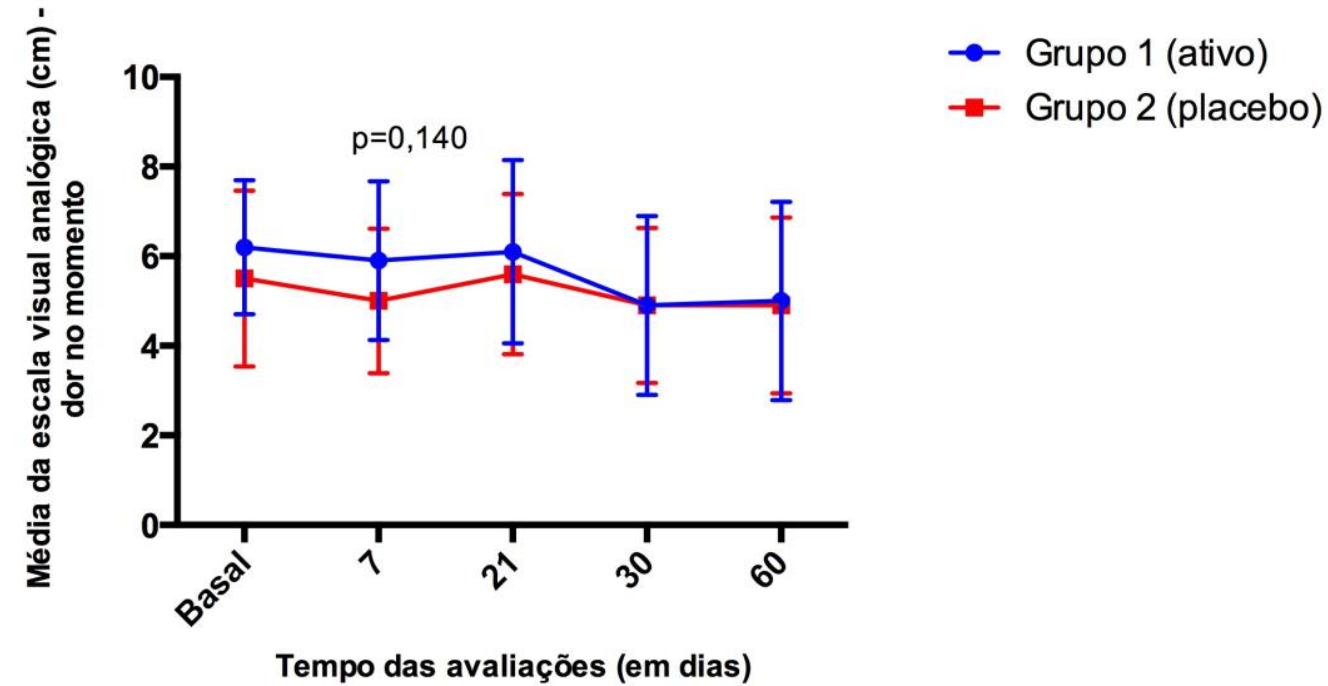

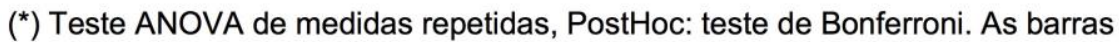
correspondem ao intervalo de confiança $95 \%$.

$\left.{ }^{* *}\right) \mathrm{BPI}=$ Inventário Breve de Dor - subescala média de dor.

Figura 42 - Escore da subescala dor no momento da avaliação do BPI dos doentes com DFA e intervalo de confiança, e resultado dos testes de associação ( $p)(\mathrm{N}=29)$. 


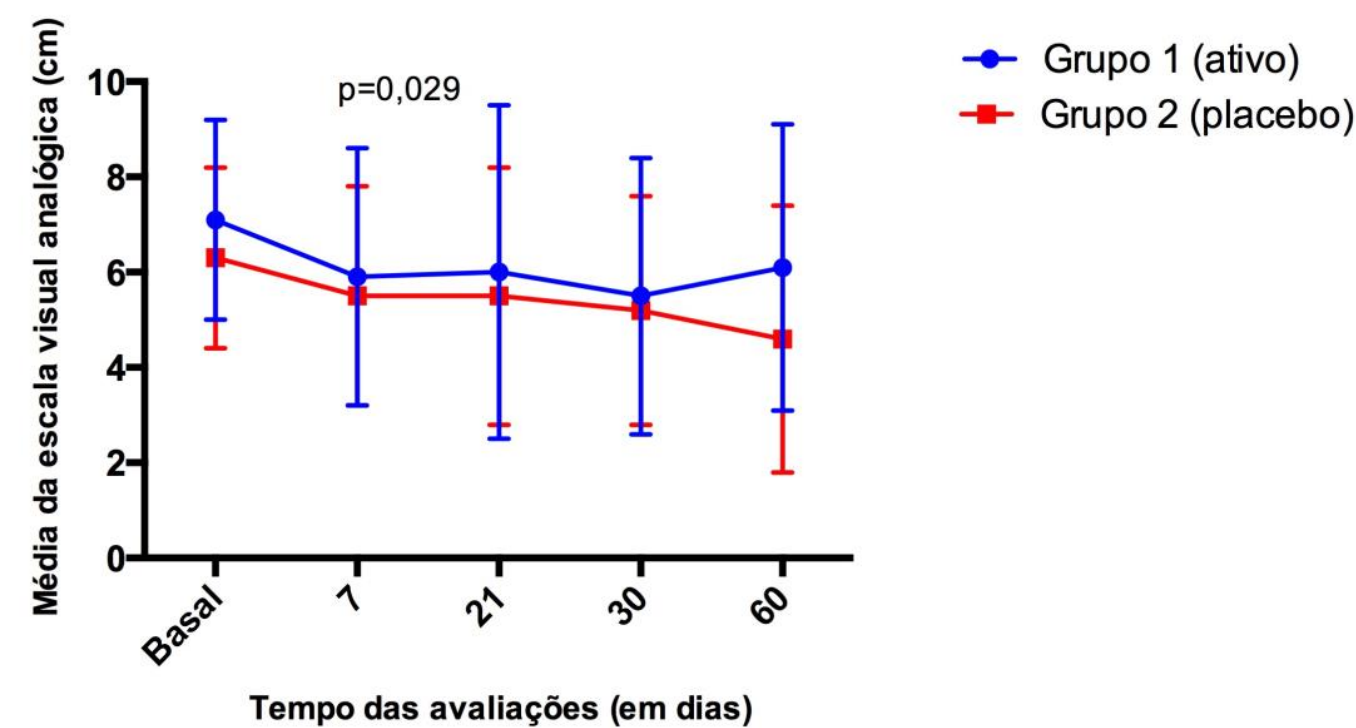

(*) Teste ANOVA de medidas repetidas, PostHoc: teste de Bonferroni. As barras correspondem ao intervalo de confiança $95 \%$.

$\left.{ }^{* *}\right) \mathrm{BPI}=$ Inventário Breve de Dor - subescala média de dor.

Figura 43 - Escore da subescala dor média nas últimas 24 horas do questionário BPI dos doentes com DFA e intervalo de confiança, e resultado dos testes de associação $(p)(N=29)$.

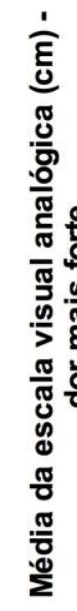

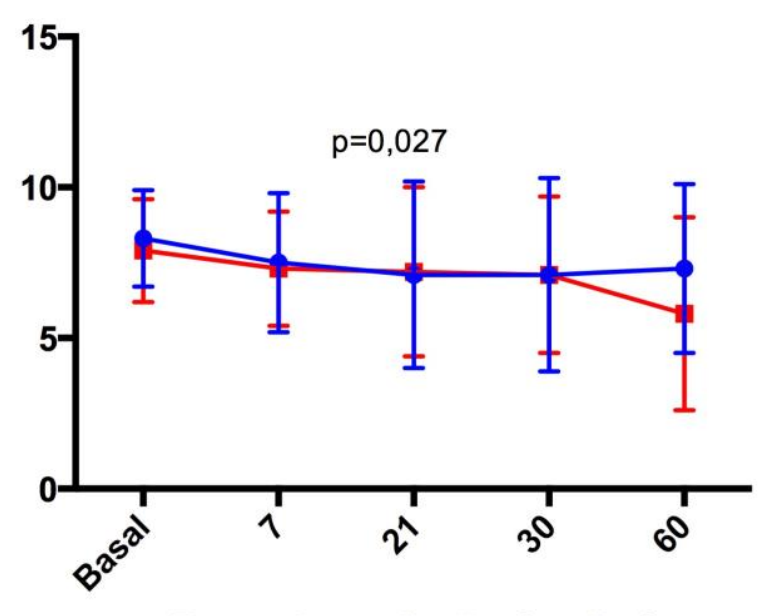

Tempo das avaliações (em dias)

$\left(^{*}\right)$ Teste ANOVA de medidas repetidas, PostHoc: teste de Bonferroni. As barras correspondem ao intervalo de confiança $95 \%$.

${ }^{(* *)} \mathrm{BPI}=$ Inventário Breve de Dor - subescala média de dor.

Figura 44 - Escore da subescala dor mais forte nas últimas 24 horas do questionário BPI dos doentes com DFA e intervalo de confiança, e resultado dos testes de associação $(p)$ $(\mathrm{N}=29)$. 
A (Figura 45) apresenta a análise comparativa através da curva de Kaplan-Meier para a melhora da dor com a EMTr. O Grupo 1 (Ativo) apresentou mediana de melhora da dor na avaliação após 30 dias (tempo de avaliação 3) (intervalo de confiança de 95\%; 0,13 a 5,86). O Grupo 2 (placebo) apresentou mediana de melhora da dor na avaliação após 21 dias (intervalo de confiança de 95\%; 1,13 a 2,86). Não houve significância estatística $\left(X^{2}=0,18, p=0,894\right)$.

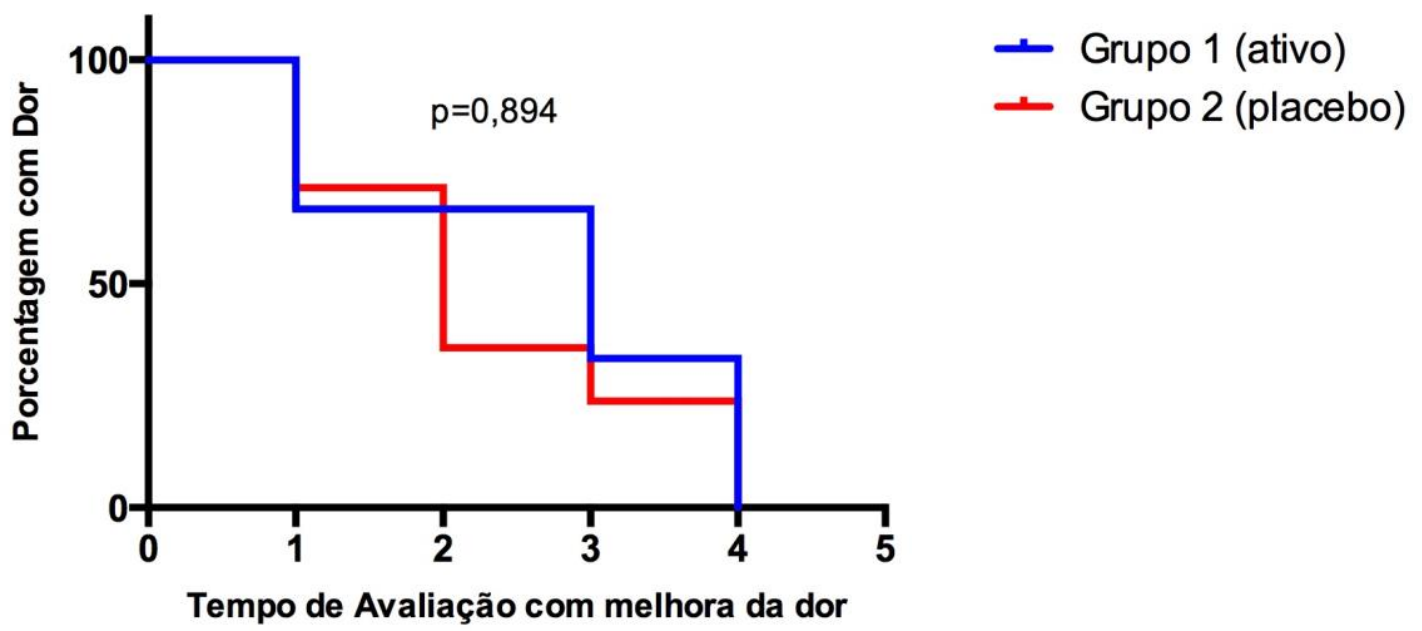

Figura 45 - Análise comparativa Curva de Kaplan-Meier entre os grupos (Ativo e Placebo) para porcentagem de melhora estimada em 30\% da EVA média dor tratada com a EMTr $(\mathrm{N}=29)$. 
6. DISCUSSÃO<smiles>C=CC=CC=CC</smiles> 
Estudos envolvendo dores craniofaciais e EMT ainda são escassos, especialmente em se tratando da DFA, condição essa que é frequentemente refratária aos tratamentos e que necessita de estudos com novas modalidades terapêuticas na busca de alternativas para seu alívio. A EMTr tem se mostrado como uma alternativa não invasiva para tratar diversas condições neuropsiquiátricas incluindo dores crônicas de origem neuropática, como fibromialgia (Mhalla, Baudic et al., 2011) e síndrome complexa regional tipo I (Picarelli, Teixeira et al., 2010). Sendo assim, este estudo investigou a utilização da EMT no tratamento da DFA com metodologia de acordo com o que foi desenvolvido pelo Centro de Dor do Departamento de Neurologia HC/FMUSP, baseando-se na literatura internacional para a produção de evidência de nível 1B (ensaio clínico controlado e randomizado com intervalo de confiança restrito) (Phillips, Ball et al., 2001).

Não houve diferença significante com relação à idade e gênero entre os grupos estudados (Siviero, Alvarez et al., 2011), havendo similaridade quanto às características gerais (idade, gênero, cor e estado civil) com outras amostras de DFA (Vickers, Cousins et al., 1998; Teixeira, Bor Seng Shu et al., 2000; Nóbrega, 2002; Prado, 2002; Agostoni, Frigerio et al., 2005; Koopman, Dieleman et al., 2009). As mulheres são mais afetadas devido a aspectos hormonais, genéticos e socioculturais (Greenspan, Craft et al., 2007; Paller, Campbell et al., 2009; Ngun, Ghahramani et al., 2011), sendo que a morbidade associada mais prevalente foi a hipertensão arterial sistêmica, o que corresponde ao esperado para os sujeitos que se 
encontram na meia idade e idosos (Siqueira, Ching et al. 2004; Siqueira 2011; Siqueira 2012; Silva 2013).

A prevalência de anormalidades odontológicas, como dentes ausentes, tipo de mordida e periodontite na amostra utilizada corresponde ao esperado na literatura (Silva, 2013), diferentemente da neuralgia idiopática do trigêmeo, que causa dor facial crônica e que pode estar associada à maior prevalência de doenças infecciosas orais devido à dificuldade de higienização quando a zona gatilho é intraoral (Siqueira, 2011; Siviero, Alvarez et al., 2011). O edentulismo pode ser consequência de procedimentos iatrogênicos que terminam em exodontias devido às dificuldades no diagnóstico diferencial da DFA (Bates Jr., Stewart, 1991; Woda, Pionchon, 1999).

Neste estudo, ambos os grupos (EMT ativo e placebo) não apresentaram diferenças estatísticas odontológicas, o que é relevante para a análise dos dados, uma vez que dores associadas a essas condições odontológicas, como pulpites e focos infecciosos, entre outros, podem interferir na percepção da dor total pelo doente, pois participam do processo de sensibilização central (Sessle, 2005; Siqueira, 2011; Siviero, Alvarez et al., 2011).

Da mesma forma, a maior frequência da dor no lado esquerdo, os fatores de piora (mudança de temperatura, estresse), fatores desencadeantes (tratamento odontológico), fatores de melhora (medicamentos, relaxamento) e a duração (média de 13,5 meses) estão de acordo com outras amostras de DFA (Brooke, 1980; Pfaffenrath, Rath et al., 
1993; Vickers, Cousins et al., 1998; Teixeira, Bor Seng Shu et al., 2000, Siqueira, Ching et al., 2004; Manzoni e Torelli, 2005; Nóbrega, Siqueira et al., 2007; Siqueira, Siqueira et al., 2012) e não diferiram entre os grupos de estudo EMT ativo e EMT placebo.

Neste estudo, as características odontológicas e álgicas não se correlacionaram com os padrões de excitabilidade cortical. Aparentemente, mecanismos neuropáticos que poderiam estar envolvidos na DFA podem estar associados a mudanças nos limiares psicofísicos de termosensibilidade (ao frio), sensibilidade mecânica táctil, sensibilidade dolorosa de superfície, dor à pressão e dor ao calor (Woda, Pionchon, 2000; List, Leijon et al., 2008; Baad-Hansen, Pigg et al., 2013), o que justificaria a queixa de piora relacionada às mudanças de temperatura (Siqueira, 2011). Terminações nervosas livres e corpúsculos de Ruffini foram encontradas alteradas no periodonto após procedimentos odontológicos, o que contribuiria para o desenvolvimento de componente neuropático na DFA (Maeda, Ochi et al., 1999).

A avaliação da presença de dor neuropática através do DN-4 e do NPSI demonstrou neste estudo que 22 doentes da amostra total apresentaram componente neuropático. Portanto, a DFA pode ser considerada composta por componentes neuropáticos e não neuropáticos, o que é compatível com a heterogeneidade do quadro clínico e que não permite classificar a origem da dor como central ou periférica. Não houve diferença entre os grupos ativo e placebo, o que poderia ter interferido nos resultados. 


\section{Comparação entre EMT ativo e EMT placebo}

Pode-se observar que, de forma geral, não houve diferença nos resultados e no alívio da dor entre os grupos ativo e placebo. Houve efeito do tempo no DN-4 entre a avaliação D21 e D7, o que poderia estar relacionado com o gerenciamento da dor crônica, do estresse, da ansiedade e da depressão (Takenoshita, Sato et al., 2010). Esses doentes apresentam morbidades associadas como quadros neuróticos, distúrbios somatoformes e estresse, bem como altos índices de catastrofização (Sullivan, Bishop, 1995) e ansiedade (Marcolino, Mathias et al., 2007). Essas condições estão associadas ao tempo de dor e ao processo de cronificação. Os achados aqui observados são similares aos encontrados em doentes com síndrome fibromiálgica com relação à ansiedade (Mhalla, Baudic et al., 2011).

Por outro lado, semelhantemente aos resultados de estudos sobre síndrome fibromiálgica (Mhalla, Baudic et al. 2011), houve diminuição gradual dos escores do BPI nessa amostra, porém não de maneira significante. Além do efeito do EMT em si, o fato do indivíduo participar de uma atividade fora de casa e manter-se ativo pode estar relacionado com essa melhora e com a redução não significante da interação BPI-EVA observada neste estudo. Uma possível justificativa para a diminuição dos escores dos questionários utilizados no estudo advém do fato da EMT influenciar a neuroplasticidade cortical, o que foi observado no córtex sensitivo de amputados (Mercier, Reilly et al., 2006). Da mesma forma, Pascual-Leone et al. (1998), demonstrou que o aumento da frequência da EMT gera um aumento da excitabilidade cortical, o que sinaliza o efeito da 
EMT em outros sistemas além do GABAérgico e dopaminérgico. Mudanças no padrão de receptores dos neurotransmissores também podem ocorrer com a EMT (Ziemann, 2004) o que modificaria a percepção geral da dor pelos doentes com DFA. Assim, pode-se justificar a redução nos escores no DN4, devido à melhora na sensibilização central dos doentes em ambos os grupos.

A avaliação através do questionário de dor McGill foi similar ao observado por Picarelli et al. (2010), no qual não houve mudança estatística nos escores. Entretanto, no estudo de Picarelli o subescore afetivo apresentou diferença, enquanto que na amostra deste estudo observou-se nesse subescore interação e efeito tempo, com efeito tempo no subescore sensitivo.

A dor crônica, de forma geral, afeta a qualidade de vida (Loduca, Samuelian et al., 2004; Siqueira, Nobrega et al., 2006; Castro, Siqueira et al., 2009). Condições que afetam o sistema estomatognático, como as disfunções temporomandibulares (Kuroiwa, Marinelli et al., 2011), podem comprometer ainda mais a vida do doente devido à importância funcional do segmento (Kandel, Schwartz et al., 2013) e à presença da dor. A necessidade de reabilitação oral também implica em problemas nutricionais, alimentares e de fala, o que afeta a saúde não só física, mas também psíquica e social (Siqueira, 2012). Os estudos correlacionando dor orofacial e qualidade de vida demonstram que a interferência na vida diária desses doentes é marcante, com alto impacto negativo independentemente do gênero. A abordagem biopsicossocial da dor tende a auxiliar no alívio dos 
sintomas e na qualidade de vida em geral (Barros, Seraidarian et al., 2009; Porto, de Leeuw et al., 2011).

Neste estudo houve diferença estatística com relação ao domínio de estado geral de saúde do questionário SF-36 entre as avaliações D30 e D21, sendo que outros domínios apresentaram melhora sem significância na análise dos dados. Essa melhora é reflexo da atenção que o doente recebeu durante o estudo pela equipe multiprofissional, o que proporcionou mudança no senso de autoeficácia devido à esperança depositada no tratamento. Isso gerou quebra no ciclo habitual de enfrentamento, o que modificou a percepção e o comportamento e promoveu melhora (Baltes, Baltes, 1991; Bandura, 1997).

\section{Excitabilidade Cortical}

Excitabilidade cortical é uma ferramenta neurofisiológica para mensurar os padrões de redes neurais presentes no córtex motor, permitindo avaliar padrões excitatórios e inibitórios (Chen, Cros et al., 2008; Rossi, Hallett et al., 2009; Groppa, Oliviero et al., 2012), e que pode ser utilizada como preditora da melhora de tratamento (Fitzgerald, Brown et al., 2004; Lefaucheur, Drout et al., 2006; Daskalakis, Christensen et al., 2008). Em geral, os sujeitos que apresentam maior variabilidade da excitabilidade cortical são aqueles que mais respondem com alívio da dor através do EMT.

Neste estudo, pode-se observar que os doentes apresentaram diferenças com relação aos controles, sendo que IIC e FIC foram maiores 
em ambos os hemisférios, diferentemente do que foi observado em doentes com síndrome fibromiálgica (diminuição da amplitude da FIC e aumento da IIC) (Mhalla, de Andrade et al., 2010). Porém, enquanto os doentes fibromiálgicos (Mhalla, de Andrade et al., 2010; Mhalla, Baudic et al., 2011) e os doentes com dor pós-acidente vascular encefálico (Hosomi, Kishima et al., 2013) tiveram restauração dos padrões normais de excitabilidade cortical. Observou-se também que os doentes do grupo ativo apresentaram aproximação de PEM 120\% e 140\% aos controles, embora sem significância estatística.

Sabe-se que os mecanismos de analgesia propiciados pela EMTr, quando aplicada sob o córtex motor, envolvem a liberação de $\mu$-opioides endógenos (Araújo, Iglesio et al., 2011; de Andrade, Mhalla et al., 2011) e é dependente de receptor NMDA (Ciampi de Andrade, Mhalla et al., 2014). Houve alívio da dor em doentes com fibromialgia (Mhalla, de Andrade et al., 2010; Mhalla, Baudic et al., 2011; Baudic, Attal et al., 2013), CRPS (Picarelli, Teixeira et al., 2010), dor visceral (Villanueva, 2011) e outras condições neuropáticas (Andre-Obadia, Peyron et al., 2006; André-Obadia, Mertens et al., 2014). Contudo, a eficiência do procedimento está relacionada ao número de sessões, frequência, intensidade e quantidade de pulsos (Galhardoni, Correia et al., 2014). Ao contrário dos estudos anteriores, que utilizaram baixo número de pulsos e poucas sessões (Defrin, Grunhaus et al., 2007), este estudo aplicou ambos em número maior. Não houve diferença entre os grupos ativo e placebo através da análise de curva de Kaplan-Meier nessa amostra. 
Fricová, Klírová et al. (2013) realizaram um estudo paralelo controlado por placebo com doentes que apresentavam dor facial crônica de diversas etiologias tais como, neuralgia do trigêmeo, neuralgia pós-herpética, cefaleia em salvas, neuralgia occipital, neuralgia local, DFA, hemicrania paroxística e neuralgia do glossofaríngeo. No resultado obtido, a estimulação de M1 a $20 \mathrm{~Hz}$ é mais efetiva que a $10 \mathrm{~Hz}$, porém ambas são melhores que placebo. Tais resultados vão em sentido oposto aos encontrados neste trabalho. Contudo, não é possível utilizar o estudo acima como referência devido à diversidade de parâmetros utilizados por ele (baixo número de pulsos - 720 pulsos a $20 \mathrm{~Hz}$ e 600 pulsos $10 \mathrm{~Hz}$-, diferentes intensidades de limiar motor $-95 \%$ a $20 \mathrm{~Hz}$ e 85,90 e $95 \%$ a $10 \mathrm{~Hz})$. Possivelmente, a resposta positiva tenha acontecido devido à heterogeneidade de doentes presentes na amostra, o que difere totalmente de nosso estudo.

Em casos publicados anteriormente, o uso da EMTr, de forma similar ao que é utilizado no tratamento da depressão, promoveu melhora significativa de doente com DFA (Reid e Pridmore, 2001; Siqueira, Marcolin et al., 2010). É possível que, no caso da DFA, devido às inúmeras morbidades psiquiátricas frequentemente associadas e ao diferente perfil desses doentes, não foi observada melhora quando utilizado protocolo de tratamento semelhante àquele que trouxe alívio da dor em diversas outras condições crônicas, como síndrome fibromiálgica, síndrome complexa de dor regional e dor central (Picarelli, Teixeira et al., 2010; Siqueira, Marcolin et al., 2010; Mhalla, Baudic et al., 2011; Hosomi, Kishima et al., 2013). 
Assim, pode-se considerar que a DFA possui componentes periféricos e centrais, além de aspectos neuropáticos e não neuropáticos, o que torna essa doença ímpar e com particularidades que devem ser consideradas no tratamento. Pelos resultados, possivelmente existem subgrupos de doentes que necessitam ser identificados para a investigação de aspectos fisiopatológicos e etiológicos associados à DFA. Por outro lado, este estudo indica que há a necessidade de seleção de outras áreas corticais ou subcorticais para estimulação, como a ínsula posterior superior direita. Essa tem se mostrado um alvo em potencial para o tratamento da dor crônica com EMTr em casos sem melhora quando utilizados alvos clássicos superficiais (Ciampi de Andrade, Galhardoni et al., 2012; Yilmaz, Kesikburun et al., 2013). 
7. CONCLUSÃO 
Através deste estudo, conclui-se que:

1) Não houve melhora da dor nos grupos ativo e placebo através da EMTr quando estimulado o córtex motor primário;

2) Os parâmetros da EMTr padronizados utilizados não foram eficazes como alternativa terapêutica para a DFA;

3) Os padrões de excitabilidade cortical (inibição e facilitação) dos doentes com DFA demonstraram-se maiores do que os padrões dos controles saudáveis. 
Agostoni E, Frigerio R, Santoro P. Atypical facial pain: clinical considerations and differential diagnosis. Neurol Sci. 2005;26 Suppl 2:s71-4.

Alvarez FK, de Siqueira SR, Okada M, Teixeira MJ, de Siqueira JT. Evaluation of the sensation in patients with trigeminal post-herpetic neuralgia. J Oral Pathol Med. 2007;36(6):347-50.

Andre-Obadia N, Peyron R, Mertens P, Mauguiere F, Laurent B, GarciaLarrea L. Transcranial magnetic stimulation for pain control. Double-blind study of different frequencies against placebo, and correlation with motor cortex stimulation efficacy. Clin Neurophysiol. 2006;117(7):1536-44.

André-Obadia N, Mertens P, Lelekov-Boissard T, Afif A, Magnin M, GarciaLarrea L. Is Life better after motor cortex stimulation for pain control? Results at long-term and their prediction by preoperative rTMS. Pain Physician. 2014 Jan-Feb;17(1):53-62.

Araújo $\mathrm{H}$, Iglesio R, Correia G, Fernandes D, Galhardoni R, Marcolin MA, et al. Estimulação magnética transcraniana e aplicabilidade clínica: perspectivas na conduta terapêutica neuropsiquiátrica. Rev Med. 2011;90(1):3-14.

Attal N, Cruccu G, Baron R, Haanpaa M, Hansson P, Jensen TS, et al. EFNS guidelines on the pharmacological treatment of neuropathic pain: 2010 revision. Eur J Neurol. 2010;17(9):1113-e88.

Baad-Hansen L, Pigg M, Ivanovic SE, Faris H, List T, Drangsholt M et al. Chairside Intraoral Qualitative Somatosensory Testing: Reliability and Comparison Between Patients with Atypical Odontalgia and Healthy Controls. Journal of Orofacial Pain. 2013;27(2):165-70.

Baltes PB, Baltes MM. Psychological perspectives on successful aging: The model of selective optimization with compensation. In: Baltes PB, Baltes MM, editors. Successful aging: A psychologic model. Cambridge: Cambridge University Press; 1991. p. 1-34.

Bandura A. Self-efficacy: the exercise of control. New York: W.H. Freeman and Company; 1997.

Barros V de M, Seraidarian PI, Cortes MI, de Paula LV. The impact of orofacial pain on the quality of life of patients with temporomandibular disorder. J Orofac Pain. 2009;23(1):28-37.

Bates Jr. RE, Stewart CM. Atypical odontalgia: phantom tooth pain. Oral Surgery, Oral Medicine, Oral Pathology. 1991;72:479-83.

Baudic S, Attal N, Mhalla A, Ciampi de Andrade D, Perrot S, Bouhassira D. Unilateral repetitive transcranial magnetic stimulation of the motor cortex does not affect cognition in patients with fibromyalgia. J Psychiatr Res. 2013;47(1):72-7.

Bayer E, Racz GB, Miles D, Heavner J. Sphenopalatine Ganglion Pulsed Radiofrequency Treatment in 30 Patients Suffering from Chronic Face and Head Pain. Pain Practice. 2005;5(3):223-7. 
Brooke RI. Atypical odontalgia: a report of twenty-two cases. Oral Surgery, Oral Medicine, Oral Pathology. 1980;49(3):196-9.

Brunoni $A$, Boggio $P$, Fregni $F$. Estimulação elétrica no sistema nervoso central: uma breve revisão histórica. In: Fregni $F$, Boggio $P$, Brunoni $A$, editors. Neuromodulação Terapêutica: princípios e avanços da estimulação cerebral não invasiva em neurologia, reabilitação, psiquiatria e neuropsicologia. São Paulo: Sarvier; 2012. p. 3-20.

Brunton L, Parker K, Blumenthal D, Buxton I. Goodman \& Gilman's: Manual of Pharmacology and Therapeutics. 4th ed. New York: McGrawHill Medical; 2008. $1230 \mathrm{p}$.

Castro AR, Siqueira SRDT, Perissinotti DMN, Teixeira MJ, Siqueira JTT. Emotional aspects of chronic orofacial pain and surgical treatment. International Journal of Surgery. 2009;7(3):196-9.

Chen R, Cros D, Curra A, Di Lazzaro V, Lefaucheur JP, Magistris MR, et al. The clinical diagnostic utility of transcranial magnetic stimulation: report of an IFCN committee. Clin Neurophysiol. 2008;119(3):504-32.

Ciampi de Andrade D, Galhardoni R, Pinto LF, Lancelotti R, Rosi J, Jr., Marcolin MA, et al. Into the island: a new technique of non-invasive cortical stimulation of the insula. Neurophysiol Clin. 2012;42(6):363-8.

Ciampi de Andrade D, Mhalla A, Adam F, Texeira MJ, Bouhassira D. Repetitive transcranial magnetic stimulation induced analgesia depends on $\mathrm{N}$-methyl-d-aspartate glutamate receptors. Pain. 2014;155(3):598-605.

Ciampi de Andrade D. Contribuição ao uso da estimulação magnética transcranianano no tratamento da dor. [Tese]. São Paulo: Faculdade de Medicina da Universidade de São Paulo; 2013.

Cornelissen P, van Kleef M, Mekhail N, Day M, van Zundert J. 3. Persistent Idiopathic Facial Pain. Pain Practice. 2009;9(6):443-8.

Costigan M, Scholz J, Woolf CJ. Neuropathic pain: a maladaptive response of the nervous system to damage. Annu Rev Neurosci. 2009;32:1-32.

Craig AD. How do you feel? Interoception: the sense of the physiological condition of the body. Nature reviews Neuroscience. 2002;3(8):655-66.

Craig AD. Interoceptive cortex in the posterior insula: comment on GarciaLarrea et al. 2010 Brain 133, 2528. Brain. 2011;134(Pt 4):e166; author reply e5.

Cruccu G, Aziz TZ, Garcia-Larrea L, Hansson P, Jensen TS, Lefaucheur JP, et al. EFNS guidelines on neurostimulation therapy for neuropathic pain. Eur J Neurol. 2007;14(9):952-70.

Cruccu G, Frisardi G, Pauletti G, Romaniello A, Manfredi M. Excitability of the central masticatory pathways in patients with painful temporomandibular disorders. Pain. 1997;73(3):447-54. 
Cruccu G, Sommer C, Anand P, Attal N, Baron R, Garcia-Larrea L, et al. EFNS guidelines on neuropathic pain assessment: revised 2009. Eur $\mathrm{J}$ Neurol. 2010;17(8):1010-8.

Daskalakis ZJ, Christensen BK, Fitzgerald PB, Moller B, Fountain SI, Chen $R$. Increased cortical inhibition in persons with schizophrenia treated with clozapine. Journal of psychopharmacology. 2008;22(2):203-9.

Davey N, Smith H, Savic G, Maskill D, Ellaway P, Frankel H. Comparison of input-output patterns in the corticospinal system of normal subjects and incomplete spinal cord injured patients. Expe Brain Res. 1999;127:382-90.

de Andrade DC, Ferreira KA, Nishimura CM, Yeng LT, Batista AF, de Sa K, et al. Psychometric validation of the Portuguese version of the Neuropathic Pain Symptoms Inventory. Health Qual Life Outcomes. 2011;9:107.

de Andrade DC, Mhalla A, Adam F, Texeira MJ, Bouhassira D. Neuropharmacological basis of rTMS-induced analgesia: the role of endogenous opioids. Pain. 2011;152(2):320-6.

Defrin R, Grunhaus L, Zamir D, Zeilig G. The effect of a series of repetitive transcranial magnetic stimulations of the motor cortex on central pain after spinal cord injury. Arch Phys Med Rehabil. 2007;88(12):1574-80.

Dworkin RH, Backonja M, Rowbotham MC, Allen RR, Argoff CR, Bennett GJ, et al. Advances in neuropathic pain: diagnosis, mechanisms, and treatment recommendations. Archives of neurology. 2003;60(11):1524-34.

Edwards MJ, Talelli P, Rothwell JC. Clinical applications of transcranial magnetic stimulation in patients with movement disorders. The Lancet Neurology. 2008;7(9):827-40.

Faul F, Erdfelder E, Lang A-G, Buchner A. G*Power 3: A flexible statistical power analysis program for the social, behavioral, and biomedical sciences. Behavior Research Methods. 2007;39(2):175-91.

Ferreira K, Dias T, Latorre M, Teixeira M, Silva A, Appolinario J. Predictors of perceived pain-related interference with emotional and physical functioning in persons with chronic pain: a population-based study. Eur J Pain Suppl. $2011 ; 5(1): 75$.

Ferreira KA, Teixeira MJ, Mendonza TR, Cleeland CS. Validation of brief pain inventory to Brazilian patients with pain. Support Care Cancer. 2011;19(4):505-11.

Ferreira KASL, de Andrade DC, Teixeira MJ. Development and Validation of a Brazilian Version of the Short-Form McGill Pain Questionnaire (SF-MPQ). Pain Management Nursing. 2011.

Fillingim RB. Individual Differences in Pain: the roles of gender, ethnicity and genetics. In: Fishman SM, Ballantyne J, Rathmell J, editors. Bonica's Management of Pain. Philadelphia: Wolters Kluwer Health; 2012. p. 86-98.

Fitzgerald PB, Brown TL, Marston NA, Daskalakis ZJ, de Castella A, Bradshaw JL, et al. Motor cortical excitability and clinical response to rTMS in depression. Journal of affective disorders. 2004;82(1):71-6. 
Fonoff ET, Dale CS, Pagano RL, Paccola CC, Ballester G, Teixeira MJ, et al. Antinociception induced by epidural motor cortex stimulation in naive conscious rats is mediated by the opioid system. Behavioural Brain Research. 2009;196(1):63-70.

Fonoff ET, Hamani C, Ciampi de Andrade D, Yeng LT, Marcolin MA, Jacobsen Teixeira $M$. Pain relief and functional recovery in patients with complex regional pain syndrome after motor cortex stimulation. Stereotactic and functional neurosurgery. 2011;89(3):167-72.

Fraizer C, Russel E. Neuralgia of the face. Arch Neurol Psychiatry. 1924;11:557-63.

Frediani F. Pharmacological therapy of atypical facial pain: actuality and perspective. Neurol Sci. 2005;26 Suppl 2:s92-4.

Fricová J, Klírová M, Masopust V, Novák T, Vérebová K, Rokyta R. Repetitive transcranial magnetic stimulation in the treatment of chronic orofacial pain. Physiol Res. 2013 Dec 12;62 Suppl 1:S125-34.

Galhardoni R, Bonfiglioli k, Ciampi de Andrade D. Fármacos utilizados no tratamento da dor. In: Fuller R, editor. Reumamecum. São Paulo: Permanyer Brasil Publicações; 2012. p. 136-73.

Galhardoni R, Correia G, Araújo H, Kaziyama HH, Marcolin MA, Bouhassira $\mathrm{D}$ et al. Repetitive transcranial Magnetic Stimulation (rTMS) in Chronic Pain. Arch Phys Med Rehabil. 2014. (Aceito para publicação).

Galhardoni R, Teixeira MJ, Siqueira SRDT, de Andrade DC. Estimulação Magnética Transcraniana repetitiva (EMTr) e dor neuropática: novas perspectivas. Dor é coisa séria. 2010;6(4):27-32.

Garcia O, Okada M, Yeng L, Teixeira M, Siqueira J, Pimenta C, et al. Atypical Facial Pain: Multidisciplinary assessment and treatment. In: 9th World Congress on Pain, August 22-27, Vienna, Austria (Abstracts). 1999.

Garcia-Larrea L. The posterior insular-opercular region and the search of a primary cortex for pain. Neurophysiol Clin. 2012;42(5):299-313.

Giamberardino MA, Affaitati G, Costantini R. Referred pain from internal organs. In: Fernando C, Troels SJ, editors. Handbook of clinical neurology. Volume 81: Elsevier; 2006. p. 343-61.

Gold M, Gebhart G. Peripheral Pain Mechanisms and Nociceptor Sensitization. In: Fishman SM, Ballantyne J, Rathmell J, editors. Bonica's Management of Pain. Philadelphia: Wolters Kluwer Health; 2012. p. 24-34.

Gonçalves DA, Dal Fabbro AL, Campos JA, Bigal ME, Speciali JG. Symptoms of temporomandibular disorders in the population: an epidemiological study. Journal of Orofacial Pain. 2010;24(3):270-8.

Graff-Radford SB, Solberg WK. Atypical Odontalgia. J Craniomandib Disord. 1992;6(4):260-5. 
Greenspan JD, Craft RM, LeResche L, Arendt-Nielsen L, Berkley KJ, Fillingim RB, et al. Studying sex and gender differences in pain and analgesia: a consensus report. Pain. 2007;132 Suppl 1:S26-45.

Groppa S, Oliviero A, Eisen A, Quartarone A, Cohen LG, Mall V, et al. A practical guide to diagnostic transcranial magnetic stimulation: Report of an IFCN committee. Clinical Neurophysiology. 2012;123(5):858-82.

Hagelberg N, Forssell H, Aalto S, Rinne JO, Scheinin H, Taiminen T, et al. Altered dopamine D2 receptor binding in atypical facial pain. Pain. 2003;106(1-2):43-8.

Hansson PT, Attal N, Baron R, Cruccu G. Toward a definition of pharmacoresistant neuropathic pain. Eur J Pain. 2009;13(5):439-40.

Harstall C, Ospina M. How Prevalent is Chronic Pain? Pain Clinical Updates - International Association for the study of Pain. 2003;XI(2):1-4.

Hirayama A, Saitoh Y, Kishima H, Shimokawa T, Oshino S, Hirata M, et al. Reduction of intractable deafferentation pain by navigation-guided repetitive transcranial magnetic stimulation of the primary motor cortex. Pain. 2006;122(1-2):22-7.

Hosomi K, Kishima H, Oshino S, Hirata M, Tani N, Maruo T, et al. Cortical excitability changes after high-frequency repetitive transcranial magnetic stimulation for central poststroke pain. Pain. 2013;154(8):1352-7.

Huerta PT, Volpe BT. Transcranial magnetic stimulation, synaptic plasticity and network oscillations. Journal of neuroengineering and rehabilitation. 2009;6:7.

International Headache Society (IHS). The International Classification of Headache Disorders, 3rd edition (beta version). Cephalalgia. 2013;33(9):629-808.

IHS. The International Classification of Headache Disorders 2nd edition. Cephalalgia. 2004;24(Suppl 1):1-150.

International Association for the Study of Pain (IASP). Classification of chronic pain. Descriptions of chronic pain syndromes and definitions of pain terms. Pain Suppl. 1986;3:S1-226.

Jääskeläinen $S K$, Forssell $H$, Tenovuo $O$. Electrophysiological testing of the trigeminofacial system: aid in the diagnosis of atypical facial pain. Pain. 1999;80:191-200.

Johanek L, Shim B, Meyer RA. Primary hyperalgesia and nociceptor sensitization. In: Fernando C, Troels SJ, editors. Handbook of clinical neurology. Volume 81: Elsevier; 2006. p. 35-47.

Kandel E, Schwartz J, Jessell T, Siegelbaum S, Hudspeth AJ. Principles of Neural Science, Fifth Edition: McGraw-Hill Education; 2013.

Khedr EM, Kotb H, Kamel NF, Ahmed MA, Sadek R, Rothwell JC. Longlasting antalgic effects of daily sessions of repetitive transcranial 
magnetic stimulation in central and peripheral neuropathic pain. Journal of Neurology, Neurosurgery, and Psychiatry. 2005;76(6):833-8.

Koopman JSHA, Dieleman JP, Huygen FJ, de Mos M, Martin CGM, Sturkenboom MCJM. Incidence of facial pain in the general population. Pain. 2009;147(1-3):122-7.

Kreisberg M. Atypical odontalgia: differential diagnosis and treatment. JADA. 1982;104:852-4.

Kuroiwa D, Marinelli J, Rampani M, Oliveira W, Nicodemo D. Desordens temporomandibulares e dor orofacial: estudo da qualidade de vida medida pelo Medical Outcomes Study 36- Item Short Form Health Survey. Revista Dor. 2011;12(2):93-8.

Lavigne G, Woda A, Truelove E, Ship JA, Dao T, Goulet JP. Mechanisms Associated with Unusual Orofacial Pain. J Orofac Pain. 2005;19:9-21.

Lefaucheur JP, Drouot X, Menard-Lefaucheur I, Keravel Y, Nguyen JP. Motor cortex rTMS restores defective intracortical inhibition in chronic neuropathic pain. Neurology. 2006;67:1568-74.

Lefaucheur JP, Hatem S, Nineb A, Menard-Lefaucheur I, Wendling S, Keravel $\mathrm{Y}$, et al. Somatotopic organization of the analgesic effects of motor cortex rTMS in neuropathic pain. Neurology. 2006;67(11):1998-2004.

Lefaucheur JP. Methods of therapeutic cortical stimulation. Neurophysiol Clin. 2009;39(1):1-14.

Lefaucheur JP. Methods of therapeutic cortical stimulation. Neurophysiol Clin. 2009;39(1):1-14.

Lefaucheur JP. Neurogenic pain relief by repetitive transcranial magnetic cortical stimulation depends on the origin and the site of pain. Journal of Neurology, Neurosurgery, and Psychiatry. 2004;75(4):612-6.

Lefaucheur JP. The use of repetitive transcranial magnetic stimulation (rTMS) in chronic neuropathic pain. Neurophysiol Clin. 2006;36(3):117-24.

List T, Leijon G, Helkimo M, Oster A, Dworkin SF, Svensson P. Clinical findings and psychosocial factors in patients with atypical odontalgia: a case control study. J Orofac Pain. 2007;21:89-98.

List T, Leijon G, Helkimo M, Öster A, Svensson P. Effect of local anesthesia on atypical odontalgia - A randomized controlled trial. Pain. 2006;122(3):306-14.

List T, Leijon G, Svensson P. Somatosensory abnormalities in atypical odontalgia: A case-control study. Pain. 2008;139(2):333-41.

Loduca A, Samuelian C, Wurzba A, Palaia R, Grimber F, Kitayama M, et al. Caracterização e representação do sofrimento de pacientes com dor crônica. Revista Dor. 2004;5(3):S:17.

Lorenz J, Hauck M. Supraspinal Mechanism of Pain and Nociception. In: Fishman SM, Ballantyne J, Rathmell J, editors. Bonica's Management of Pain. Philadelphia: Wolters Kluwer Health; 2012. p. 62-74. 
Maeda T, Ochi K, Nakakura-Ohshima K, Youn S, Wakisaka S. The Ruffini ending as the primary mechanoreceptor in the periodontal ligament: its morphology, cytochemical features, regeneration, and development. Critical Reviews in Oral Biology \& Medicine. 1999;10(3):307-27.

Manzoni GC, Torelli P. Epidemiology of typical and atypical craniofacial neuralgias. Neurol Sci. 2005;26 Suppl 2:s65-7.

Marbach JJ. Phantom tooth pain. J Endod. 1978;4:362-72.

Marcolino J, Mathias L, Piccinini Fo L, Guaratini A, Suzuki F, Alli L. Escala Hospitalar de Ansiedade e Depressão: Estudo de Validade de Critério e da Confiabilidade com pacientes no pré-operatório. Rev Bras Anestesiol. 2007;57(1):52-62.

McMillan AS, Graven-Nielsen T, Romaniello A, Svensson P. Motor potentials evoked by transcranial magnetic stimulation during isometric and dynamic masseter muscle contraction in humans. Archives of oral biology. 2001;46(4):381-6.

Melis M, Lobo SL, Ceneviz C, Zawawi K, Al-Badawi E, Maloney G, et al. Atypical odontalgia: a review of the literature. Headache. 2003;43:1060-74.

Menezes Costa Lda C, Maher CG, McAuley JH, Hancock MJ, de Melo Oliveira W, Azevedo DC, et al. The Brazilian-Portuguese versions of the McGill Pain Questionnaire were reproducible, valid, and responsive in patients with musculoskeletal pain. Journal of clinical epidemiology. 2011;64(8):903-12.

Mercier C, Reilly KT, Vargas CD, Aballea A, Sirigu A. Mapping phantom movement representations in the motor cortex of amputees. Brain. 2006;129(Pt 8):2202-10.

Merskey $\mathrm{H}$, Bogduk N. Classification of chronic pain: description of chronic pain syndromes and defnitions of pain terms. 2nd ed. Seattle: IASP Press; 1994.

Mhalla A, Baudic S, Ciampi de Andrade D, Gautron M, Perrot S, Teixeira MJ, et al. Long-term maintenance of the analgesic effects of transcranial magnetic stimulation in fibromyalgia. Pain. 2011;152(7):1478-85.

Mhalla A, de Andrade DC, Baudic S, Perrot S, Bouhassira D. Alteration of cortical excitability in patients with fibromyalgia. Pain. 2010;149(3):495-500.

Nader A, Kendall MC, De Oliveira Jr. GS, Chen JQ, Vanderby B, Rosenow $\mathrm{JM}$, et al. Ultrasound-Guided Trigeminal Nerve Block via the Pterygopalatine Fossa: An Effective Treatment for Trigeminal Neuralgia and Atypical Facial Pain. Pain Physician. 2013;16:E537-E45.

Nasri C, Siqueira J. Síndrome da Ardência Bucal. In: Siqueira J, Teixeira MJ, editors. Dores Orofaciais: diagnóstico e tratamento. 1. São Paulo: Artes Médicas; 2012. p. 293-309.

Ngun TC, Ghahramani N, Sanchez FJ, Bocklandt S, Vilain E. The genetics of sex differences in brain and behavior. Frontiers in neuroendocrinology. 2011;32(2):227-46. 
Nóbrega JCM, Siqueira SRDT, Siqueira J, Teixeira MJ. Differential Diagnosis In Atypical Facial Pain. Arq Neuropsiquiatr. 2007;65(2-A):256-61.

Nóbrega JCM, Teixeira MJ, Corrêa CF, Okada M. Nucleotratotomia trigeminal estereotáxica no tratamento da dor facial atípica. Arq Bras Neurocir. 2000;19(3):131-5.

Nóbrega JCM. Dor facial atípica e dor facial sintomática: caracterização diferencial quanto à apresentação clínica. [Dissertação]. São Paulo: Universidade Federal de São Paulo; 2002.

Nordstrom MA. Insights into the bilateral cortical control of human masticatory muscles revealed by transcranial magnetic stimulation. Archives of oral biology. 2007;52(4):338-42.

Oliveira L. Princípios gerais do tratamento farmacológico da dor. In: Alves Neto O, Costa C, Siqueira J, Teixeira MJ, editors. Dor: princípios e prática. Porto Alegre: Artmed; 2009. p. 1033-41.

Pagano RL, Assis DV, Clara JA, Alves AS, Dale CS, Teixeira MJ, et al. Transdural motor cortex stimulation reverses neuropathic pain in rats: $A$ profile of neuronal activation. Eur J Pain. 2011;15(3):268.e1-.e14.

Pagano RL, Fonoff ET, Dale CS, Ballester G, Teixeira MJ, Britto LRG. Motor cortex stimulation inhibits thalamic sensory neurons and enhances activity of PAG neurons: Possible pathways for antinociception. Pain. 2012;153(12):2359-69.

Paller CJ, Campbell CM, Edwards RR, Dobs AS. Sex-based differences in pain perception and treatment. Pain Med. 2009;10(2):289-99.

Pascual-Leone A, Tormos JM, Keenan J, Tarazona F, Canete C, Catala MD. Study and modulation of human cortical excitability with transcranial magnetic stimulation. Journal of clinical neurophysiology : official publication of the American Electroencephalographic Society. 1998;15(4):333-43.

Pearce SL, Miles TS, Thompson PD, Nordstrom MA. Responses of single motor units in human masseter to transcranial magnetic stimulation of either hemisphere. J Physiol. 2003;549(Pt 2):583-96.

Perera R, Heneghan C, Bandenoch D. Ferramentas Estatísticas no contexto clínico. Porto Alegre: Artmed; 2010. 120 p.

Pfaffenrath V, Rath M, Pöllman W, Keeser W. Atypical facial pain-application of the IHS criteria in a clinical sample. Cephalalgia. 1993;13(suppl 12):84-8.

Phillips B, Ball C, al. SDe. Oxford Centre for evidence-based Medicine Levels of Evidence Grades of Recommendation 2001 [cited 201309 march]. Available from: Disponível em: http:// cebm.jr2.ox.ac.uk/docs/ levels4.html.

Picarelli H, Teixeira MJ, de Andrade DC, Myczkowski ML, Luvisotto TB, Yeng LT, et al. Repetitive transcranial magnetic stimulation is efficacious as an add-on to pharmacological therapy in complex regional pain syndrome (CRPS) type I. J Pain. 2010;11(11):1203-10. 
Porto F, de Leeuw R, Evans DR, Carlson CR, Yepes JF, Branscum A, et al. Differences in psychosocial functioning and sleep quality between idiopathic continuous orofacial neuropathic pain patients and chronic masticatory muscle pain patients. J Orofac Pain. 2011;25(2):117-24.

Prado E. Dor facial atípica: caracterização de uma amostra baseada na avaliação multidisciplinar; proposta de classificação baseada em estudo clínico e em exames complementares. [Dissertação]. São Paulo: Universidade de São Paulo; 2002.

Ram S, Teruel A, Kumar SKS, Clark G. Clinical Characteristics and diagnosis of Atypical Odontalgia: Implications for dentist. JADA. 2009;140 (2):223-8.

Rees RT, Harris M. ATYPICAL ODONTALGIA. British Journal Of Oral Surgery. 1978-79;16:212-8.

Reid P, Pridmore S. Improvement i Chronic pain with transcranial magnetic stimulation. Aust N Z J Pychiatry. 2001;35(2):252.

Reik Jr. L. Atypical odontalgia: a localized form of atypical facial pain. Headache. 1984;24:222-4.

Rossi S, Hallett M, Rossini PM, Pascual-Leone A. Safety, ethical considerations, and application guidelines for the use of transcranial magnetic stimulation in clinical practice and research. Clin Neurophysiol. 2009;120(12):2008-39.

Sa KN, Baptista AF, Matos MA, Lessa I. Chronic pain and gender in Salvador population, Brazil. Pain. 2008;139(3):498-506.

Salerno A, Thomas E, Olive P, Blotman F, Picot MC, Georgesco M. Motor cortical dysfunction disclosed by single and double magnetic stimulation in patients with fibromyalgia. Clin Neurophysiol. 2000;111(6):994-1001.

Santos JG, Brito JO, de Andrade DC, Kaziyama VM, Ferreira KA, Souza I, et al. Translation to Portuguese and validation of the Douleur Neuropathique 4 questionnaire. J Pain. 2010;11(5):484-90.

Schnurr RF, Brooke RI. Atypical odontalgia Update and comment on longterm follow-up. Oral Surgery, Oral Medicine, Oral Pathology. 1992;73:445-8.

Sessle BJ. Peripheral and central mechanisms of orofacial pain and their clinical correlates. Minerva Anestesiol. 2005;71(4):117-36.

Shanthanna $\mathrm{H}$. Utility of stellate ganglion block in atypical facial pain: a case report and consideration of its possible mechanisms. Case Rep Med. 2013;2013:293826.

Silva LAd. Avaliação trigeminal somestésica, gustativa, olfativa e salivar em diferentes faixas etárias: Universidade de São Paulo; 2013.

Siqueira J, Ching LH, Nasri C, Siqueira SRDT, Teixeira MJ, Heir G, et al. Clinical study of patients with persistent orofacial pain. Arq Neuropsiquiatr. 2004;62(4):988-96. 
Siqueira J, Marcolin MA, Teixeira MJ, Siqueira S. Persistent atypical odontalgia treated with transcranial magnetic stimulation. Case report Rev Dor. 2010;11(3):259-61.

Siqueira J, Siqueira SRDT, Teixeira MJ. Dor Facial Atípica/Odontalgia Atípica. In: Siqueira J, Teixeira MJ, editors. Dores Orofaciais: diagnóstico e tratamento. São Paulo: Artes Médicas; 2012. p. 409-16.

Siqueira J. Avaliação da dor orofacial persistente e exames complementares em pacientes complexos ou com doenças sistêmicas. In: Siqueira J, Teixeira MJ, editors. Dores orofaciais. São Paulo: Artes Médicas; 2012. p. 145-58.

Siqueira J. Odontalgias odontogênicas e não odontogênicas. In: Siqueira J, Teixeira MJ, editors. Dores Orofaciais: diagnóstico e tratamento. 1. São Paulo: Artes Médicas; 2012. p. 337-51.

Siqueira J. Pesquisa, ensino e asistência em dor orofacial. In: Siqueira J, Teixeira M, editors. Dores Orofaciais. 1를 ed. São Paulo: Artes Médicas; 2012. p. 17-37.

Siqueira J. Reabilitação Oral e Qualidade de Vida. In: Siqueira J, Teixeira MJ, editors. Dores orofaciais. São Paulo: Artes Médicas; 2012. p. 795-810.

Siqueira SRDT. Avaliação sensitiva quantitativa de doentes com dor orofacial. [Tese]. São Paulo: Faculdade de Medicina da Universidade de São Paulo; 2011.

Siqueira SRDT, da Nobrega JC, de Siqueira JT, Teixeira MJ. Frequency of postoperative complications after balloon compression for idiopathic trigeminal neuralgia: prospective study. Oral Surg Oral Med Oral Pathol Oral Radiol Endod. 2006;102(5):e39-45.

Siqueira SRDT, Teixeira MJ. Neuralgia Idiopática do Trigêmeo. In: Siqueira J, Teixeira MJ, editors. Dores Orofaciais: diagnóstico e tratamento. 1. São Paulo: Artes Médicas; 2012. p. 396-408.

Siviero M, Alvarez FK, Okada M, Teixeira MJ, de Siqueira JT, de Siqueira SR. Facial sensibility of patients with trigeminal neuralgias. Clinical neurology and neurosurgery. 2011;113(4):268-71.

Stump P. Orientação para o tratamento farmacológico da dor neuropática. Dor é coisa séria. 2012;8(2):5-16.

Sullivan M, Bishop S. The Pain Catastrophizing Scale: Development and Validation. Psychological Assessment. 1995;7(4):524-32.

Takenoshita M, Sato T, Kato Y, Katagiri A, Yoshikawa T, Sato $Y$, et al. Psychiatric diagnoses in patients with burning mouth syndrome and atypical odontalgia referred from psychiatric to dental facilities. Neuropsychiatr Dis Treat. 2010(6):699-705.

Teixeira M. Fisiopatologia da dor. In: Alves Neto O, Costa C, Siqueira J, Teixeira MJ, editors. Dor: princípios e prática. 1. Porto Alegre: Artmed; 2009. p. $145-75$. 
Teixeira MJ, Bor Seng Shu E, Nóbrega JCM, Corrêa CF. Dor Facial atípica: caracterização de uma amostra Arq Bras Neurocir. 2000;19(2):64-70.

Teixeira MJ, Kraychete D, Guimarães C, Carvalho MG. Epidemiologia Clínica da Dor. In: Teixeira MJ, editor. Dor: Manual para o Clínico. São Paulo: Ed. Atheneu; 2006. p. 1-8.

Teixeira MJ, Okada M. Neuralgia pós-herpética. Rev Med. 1999;78(3):140-9.

Teixeira MJ, Siqueira J, Alvarez F. Fisiopatologia da dor/glóssario de termos para a semiologia da dor. In: Siqueira J, Teixeira MJ, editors. Dores orofaciais: diagnóstico e tratamento. São Paulo: Artes Médicas; 2012b. p. 61-81.

Teixeira MJ, Siqueira SRDT, Kosminsky M, Monteiro AA. Epidemiologia da Dor. In: Siqueira J, Teixeira M, editors. Dores Orofaciais. 1르 ed. São Paulo: Artes Médicas; 2012a. p. 46-60.

Todd AJ. Anatomy and neurochemistry of the dorsal horn. In: Fernando C, Troels SJ, editors. Handbook of clinical neurology. Volume 81: Elsevier; 2006. p. 61-76.

Trace M, Barbieri C, Sardella A. Atypical odontalgia: an up-to-date view. Minerva Stomatol. 2013;62(5):163-81.

Tracey I, Mantyh PW. The cerebral signature for pain perception and its modulation. Neuron. 2007;55(3):377-91.

Tsubokawa T, Katayama Y, Yamamoto T, Hirayama T, Koyama S. Treatment of thalamic pain by chronic motor cortex stimulation. PACE. 1991;14:131-4.

Turner L, Shamseer L, Altman DG, Weeks L, Peters J, Kober T, et al. Consolidated standards of reporting trials (CONSORT) and the completeness of reporting of randomised controlled trials (RCTs) published in medical journals. The Cochrane database of systematic reviews. 2012;11:Mr000030.

Twaddle M, Cooke KJ. Assessment of Pain \& Common Pain Syndromes. In: Von Roenn J, Paice J, Preodor M, editors. Current Diagnosis \& Treatment of Pain: McGraw-Hill Education; 2006. p. 10-20.

Vickers ER, Cousins MJ, Walker S, Chisholm K. Analysis of 50 patients with atypical odontalgia. A preliminary report on pharmacological procedures for diagnosis and treatment. Oral Surgery, Oral Medicine, Oral Pathology. 1998;85:24-32.

Villanueva L. Repetitive transcranial magnetic stimulation (rTMS) as a tool for the treatment of chronic visceral pain. Eur J Pain. 2011;15(1):1-2.

Von Roenn J, Paice J, Preodor M. Current Diagnosis \& Treatment of Pain: McGraw-Hill Education; 2006.

Ward H, Azzaro A. Drogas usadas nos distúrbios de humor. In: Craig C, Stitzel R, editors. Farmacologia moderna com aplicações clínicas. 6ed. Rio de Janeiro: Guanabara Koogan; 2005. p. 361-71. 
Wirz S, Wartenberg HC, Nadstawek J. Pain management procedures used by dental and maxillofacial surgeons: an investigation with special regard to odontalgia. Head Face Med. 2005;1:14.

Woda A, Pionchon P. A Unified Concept of Idiopathic Orofacial Pain: Clinical Features. J Orofac Pain. 1999;13:172-84.

Woda A, Pionchon P. A Unified Concept of Idiopathic Orofacial Pain: Pathophysiologic Features. J Orofac Pain. 2000;14:196-212.

Woda A. Neuropathic Pain. J Orofac Pain. 2007;22(3):257-8.

Woda A. Pain in the Trigeminal System: from Orofacial Nociception to Neural Network Modeling. j dent res. 2003;82(10):764-8.

Wood JN. Molecular mechanisms of nociception and pain. In: Fernando C, Troels SJ, editors. Handbook of clinical neurology. Volume 81: Elsevier; 2006. p. 49-59.

YIImaz B, Kesikburun S, Yas Ar E, Tan AK. The effect of repetitive transcranial magnetic stimulation on refractory neuropathic pain in spinal cord injury. J Spinal Cord Med. 2013 Nov 11. [Epub ahead of print]

Zagury JG, Eliav E, Heir GM, Nasri-Heir C, Ananthan S, Pertes R, et al. Prolonged gingival cold allodynia: a novel finding in patients with atypical odontalgia. Oral Surg Oral Med Oral Pathol Oral Radiol Endod. 2011;111(3):312-9.

Zakrzewska JM. Facial pain: an update. Curr Opin Support Palliat Care. 2009;3(2):125-30.

Zhang J, Baccei M. Pathophysiology of Pain. In: Smith HS, editor. Current Therapy in Pain: Saunders/Elsevier; 2008. p. 3-8.

Ziemann U. TMS and drugs. Clin Neurophysiol. 2004;115(8):1717-29. 
Tabela 15 - Excitabilidade cortical dos doentes com DFA comparados aos controles saudáveis em médias e desvio padrão, e resultados dos testes de associação (p) $(\mathrm{N}=29)$

\begin{tabular}{|c|c|c|c|c|c|}
\hline \multirow[b]{3}{*}{ Categoria } & \multicolumn{4}{|c|}{ Grupos* } & \multirow[b]{3}{*}{$\mathbf{p}^{\star *}$} \\
\hline & \multicolumn{2}{|c|}{ DFA } & \multicolumn{2}{|c|}{ Controles } & \\
\hline & $\mathrm{N}=29$ & DP & $\mathrm{N}=28$ & DP & \\
\hline LMR_D (\%)\# & 25,58 & 5,67 & 26,50 & 8,92 & 0,974 \\
\hline PEM_120\% $(\mu \mathrm{V})$ & 174,91 & 207,24 & 276,58 & 266,57 & 0,114 \\
\hline PEM_140\% $(\mu \mathrm{V})$ & 227,84 & 332,65 & 342,68 & 293,80 & 0,053 \\
\hline IIC_D $(\mu \mathrm{V})$ & 1,62 & 1,18 & 0,35 & 0,25 & 0,000 \\
\hline FIC-D $(\mu \mathrm{V})$ & 1,76 & 1,34 & 0,54 & 0,34 & 0,000 \\
\hline Razão 140/120_D ( $\mu \mathrm{V})$ & 1,51 & 0,811 & 1,52 & 1,18 & 0,453 \\
\hline LMR_E (\%) & 25,79 & 6,38 & 26,03 & 7,66 & 0,898 \\
\hline PEM $120 \%(\mu \mathrm{V})$ & 185,61 & 329,81 & 285,22 & 370,15 & 0,032 \\
\hline PEM_140\% $(\mu \mathrm{V})$ & 294,08 & 485,11 & 294,72 & 415,69 & 0,260 \\
\hline IIC_E $\bar{E}(\mu \mathrm{V})$ & 1,56 & 1,27 & 0,35 & 0,30 & 0,000 \\
\hline FIC_E $(\mu \mathrm{V})$ & 1,70 & 1,68 & 0,52 & 0,35 & 0,000 \\
\hline Razão 140/120_E ( $\mu \mathrm{V})$ & 3,31 & 6,67 & 1,30 & 1,08 & 0,021 \\
\hline
\end{tabular}

**Teste $U$ de Mann-Whitney \#LMR = Limiar motor em repouso, PEM= potencial evocado motor, IIC = inibição intracortical, FIC = facilitação intracortical; D: direito, E: esquerdo

Tabela 16 - Excitabilidade cortical de acordo com o grupo em médias e desvio padrão, e resultados dos testes de associação (p) no tempo D7 (N=29)

\begin{tabular}{|c|c|c|c|c|c|}
\hline \multirow[b]{3}{*}{ Categorias } & \multicolumn{4}{|c|}{ Grupos* } & \multirow[b]{3}{*}{$\mathbf{p}^{\star \star}$} \\
\hline & \multicolumn{2}{|c|}{ Grupo1 (Ativo) } & \multicolumn{2}{|c|}{ Grupo 2 (Placebo) } & \\
\hline & $\mathrm{N}=15$ & DP & $\mathrm{N}=14$ & DP & \\
\hline LMR D (\%) & 23,33 & 5,70 & 23,12 & 4,42 & 0,880 \\
\hline PEM_120\% $(\mu \mathrm{V})$ & 326,56 & 556,66 & 138,61 & 167,78 & 0,217 \\
\hline PEM_-140\% $(\mu \mathrm{V})$ & 494,42 & 778,14 & 154,28 & 169,60 & 0,172 \\
\hline IIC_D $(\mu \mathrm{V})$ & 1,32 & 1,01 & 1,40 & 0,77 & 0,505 \\
\hline FIC_D $(\mu V)$ & 1,59 & 1,55 & 1,78 & 1,50 & 0,880 \\
\hline $\begin{array}{l}\text { Razão 140/120_D } \\
(\mu \mathrm{V})\end{array}$ & 2,25 & 3,89 & 2,79 & 5,95 & 0,747 \\
\hline LMR_E (\%) & 24,33 & 4,95 & 23,64 & 3,99 & 0,914 \\
\hline PEM_120\% $(\mu \mathrm{V})$ & 181,02 & 143,74 & 126,85 & 77,29 & 0,354 \\
\hline PEM_140\% $(\mu \mathrm{V})$ & 189,91 & 134,18 & 157,51 & 143,25 & 0,477 \\
\hline IIC_E $(\mu \mathrm{V})$ & 1,22 & 0,50 & 1,76 & 2,09 & 0,683 \\
\hline FIC_E $(\mu V)$ & 1,62 & 1,77 & 1,55 & 1,93 & 0,780 \\
\hline Razão 140/120_E ( $\mu \mathrm{V})$ & 1,50 & 1,30 & 1,31 & 0,72 & 0,949 \\
\hline
\end{tabular}

${ }^{\star *}$ Teste Kruskal-Wallis. LMR = Limiar motor em repouso, $\mathrm{PEM}=$ potencial evocado motor, IIC = inibição intracortical, FIC = facilitação intracortical. D: Direito, E: Esquerdo. DP: Desvio Padrão. 
Tabela 17 - Excitabilidade cortical de acordo com o grupo em médias e desvio padrão, e resultados dos testes de associação (p) no tempo D21 ( $N=29)$

\begin{tabular}{|c|c|c|c|c|c|}
\hline \multirow[b]{3}{*}{ Categorias } & \multicolumn{4}{|c|}{ Grupos* } & \multirow[b]{3}{*}{$p^{\star \star}$} \\
\hline & \multicolumn{2}{|c|}{$\begin{array}{c}\text { Grupo } 1 \\
\text { (Ativo) }\end{array}$} & \multicolumn{2}{|c|}{$\begin{array}{c}\text { Grupo } 2 \\
\text { (Placebo) }\end{array}$} & \\
\hline & $\mathrm{N}=15$ & DP & $\mathrm{N}=14$ & DP & \\
\hline LMR_D (\%) & 23,46 & $\pm 3,73$ & 23,14 & $\pm 4,70$ & 0,813 \\
\hline PEM_120\% $(\mu \mathrm{V})$ & 168,26 & $\pm 141,92$ & 117,24 & $\pm 126,21$ & 0,102 \\
\hline PEM_-140\% $(\mu \mathrm{V})$ & 194,72 & $\pm 193,49$ & 180,91 & $\pm 213,49$ & 0,331 \\
\hline IIC_D $(\mu \mathrm{V})$ & 1,16 & $\pm 0,51$ & 1,28 & $\pm 0,86$ & 0,914 \\
\hline FIC_D $(\mu \mathrm{V})$ & 1,08 & $\pm 0,42$ & 1,21 & $\pm 0,47$ & 0,533 \\
\hline Razão 140/120_D $(\mu \mathrm{V})$ & 1,62 & $\pm 1,92$ & 1,76 & $\pm 1,61$ & 1,000 \\
\hline LMR_E (\%) & 24,06 & $\pm 4,69$ & 23,78 & $\pm 6,02$ & 0,621 \\
\hline PEM_120\% $(\mu \mathrm{V})$ & 262,48 & $\pm 288,46$ & 80,65 & $\pm 60,90$ & 0,005 \\
\hline PEM_140\% $(\mu \mathrm{V})$ & 270,16 & $\pm 393,69$ & 210,40 & $\pm 431,57$ & 0,102 \\
\hline IIC E $(\mu \mathrm{V})$ & 1,20 & $\pm 0,54$ & 1,43 & $\pm 0,79$ & 0,715 \\
\hline FIC_E $(\mu \mathrm{V})$ & 1,12 & $\pm 0,46$ & 1,35 & $\pm 1,04$ & 0,949 \\
\hline Razão 140/120_E $(\mu \mathrm{V})$ & 0,95 & $\pm 0,52$ & 2,31 & $\pm 3,21$ & 0,023 \\
\hline
\end{tabular}

${ }^{* *}$ Teste Kruskal-Wallis. LMR = Limiar motor em repouso, $\mathrm{PEM}=$ potencial evocado motor, IIC = inibição intracortical, FIC = facilitação intracortical. D: Direito, E: Esquerdo. DP: Desvio Padrão.

Tabela 18 - Excitabilidade cortical de acordo com o grupo em médias e desvio padrão, e resultados dos testes de associação (p) no tempo D30 ( $N=29)$

\begin{tabular}{|c|c|c|c|c|c|}
\hline \multirow[b]{3}{*}{ Categorias } & \multicolumn{4}{|c|}{ Grupos* } & \multirow[b]{3}{*}{$\mathbf{p}^{\star *}$} \\
\hline & \multicolumn{2}{|c|}{ Grupo 1 (Ativo) } & \multicolumn{2}{|c|}{ Grupo 2 (Placebo) } & \\
\hline & $\mathrm{N}=15$ & DP & $\mathrm{N}=14$ & DP & \\
\hline LMR_D (\%) & 24,00 & $\pm 4,64$ & 23,35 & $\pm 3,58$ & 0,505 \\
\hline PEM $120 \%(\mu \mathrm{V})$ & 204,38 & $\pm 178,76$ & 127,77 & $\pm 91,37$ & 0,290 \\
\hline PEM $140 \%(\mu \mathrm{V})$ & 226,55 & $\pm 158,79$ & 136,60 & $\pm 126,12$ & 0,051 \\
\hline IIC_D $(\mu \mathrm{V})$ & 1,29 & $\pm 1,08$ & 1,32 & $\pm 0,65$ & 0,715 \\
\hline FIC $D(\mu V)$ & 1,18 & $\pm 0,90$ & 1,32 & $\pm 0,90$ & 0,780 \\
\hline Razão 140/120_D ( $\mu \mathrm{V})$ & 1,26 & $\pm 0,61$ & 1,13 & $\pm 0,46$ & 1,000 \\
\hline LMR_E (\%) & 23,13 & $\pm 3,52$ & 23,35 & $\pm 4,78$ & 0,880 \\
\hline PEM_120\% $(\mu \mathrm{V})$ & 159,35 & $\pm 178,15$ & 136,28 & $\pm 89,29$ & 0,847 \\
\hline PEM $140 \%(\mu \mathrm{V})$ & 155,02 & $\pm 99,68$ & 154,98 & $\pm 120,67$ & 0,847 \\
\hline IIC $\bar{E}(\mu \mathrm{V})$ & 1,01 & $\pm 0,50$ & 2,01 & $\pm 1,89$ & 0,290 \\
\hline FIC $E(\mu V)$ & 1,45 & $\pm 1,55$ & 1,83 & $\pm 1,78$ & 0,533 \\
\hline Razão 140/120_E $(\mu \mathrm{V})$ & 1,53 & $\pm 0,93$ & 1,38 & $\pm 0,84$ & 0,780 \\
\hline
\end{tabular}

**Teste Kruskal-Wallis. LMR = Limiar motor em repouso, PEM= potencial evocado motor, IIC = inibição intracortical, FIC = facilitação intracortical. D: Direito, E: Esquerdo. DP: Desvio Padrão. 
Tabela 19 - Excitabilidade cortical de acordo com o grupo em médias e desvio padrão, e resultados dos testes de associação (p) no tempo D60 ( $N=29)$

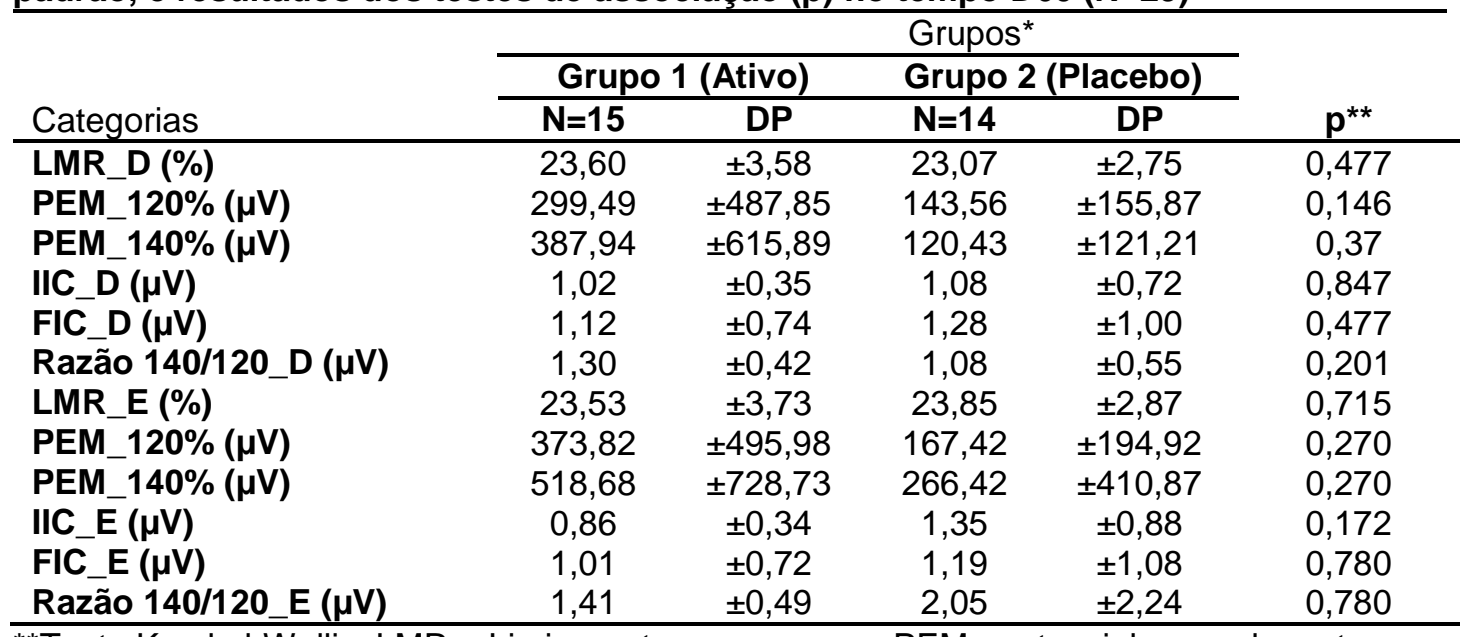

**Teste Kruskal-Wallis. LMR = Limiar motor em repouso, $\mathrm{PEM}=$ potencial evocado motor, IIC = inibição intracortical, FIC = facilitação intracortical. D: Direito, E: Esquerdo. DP: Desvio Padrão.

Tabela 20 - Avaliação dos doentes através do NPSI de acordo com o grupo e com o período em médias e desvio padrão, e resultados dos testes de associação ( $p$ ) ( $N=29)$

\begin{tabular}{|c|c|c|c|c|c|c|c|c|c|}
\hline \multirow[b]{3}{*}{ Característica } & \multirow[b]{3}{*}{ Categorias } & \multicolumn{4}{|c|}{ Grupos* } & \multicolumn{4}{|c|}{ Medidas de efeito** } \\
\hline & & \multicolumn{2}{|c|}{$\begin{array}{c}\text { Grupo } 1 \\
\text { (Ativo) }\end{array}$} & \multicolumn{2}{|c|}{$\begin{array}{c}\text { Grupo } 2 \\
\text { (Placebo) }\end{array}$} & \multirow{2}{*}{$\begin{array}{c}\begin{array}{c}\text { Basal- } \\
\text { D7 }\end{array} \\
p\end{array}$} & \multirow{2}{*}{$\begin{array}{c}\text { D7- } \\
\text { D21 } \\
p\end{array}$} & \multirow{2}{*}{$\begin{array}{c}\text { D21- } \\
\text { D30 } \\
p\end{array}$} & \multirow{2}{*}{$\begin{array}{c}\text { D30- } \\
\text { D60 } \\
p\end{array}$} \\
\hline & & $\mathrm{N}=15$ & DP & $\mathrm{N}=14$ & DP & & & & \\
\hline \multirow[t]{7}{*}{ NPSI } & Basal & 47,13 & $\pm 17,12$ & 39,64 & $\pm 23,48$ & & & & \\
\hline & D7 & 40,73 & $\pm 27,40$ & 35,78 & $\pm 21,45$ & & & & \\
\hline & D21 & 38,00 & $\pm 26,98$ & 36,14 & $\pm 25,40$ & & & & \\
\hline & D30 & 36,60 & $\pm 28,33$ & 31,85 & $\pm 22,98$ & & & & \\
\hline & $\mathrm{D} 60$ & 35,40 & $\pm 25,45$ & 30,14 & $\pm 24,64$ & & & & \\
\hline & Ef. Tempo & & & & & 0,191 & 0,747 & 0,228 & 0,531 \\
\hline & Interação & & & & & 0,742 & 0,675 & 0,537 & 0,912 \\
\hline
\end{tabular}

\footnotetext{
${ }^{*}$ Análise de variância para medidas repetidas. NPSI: Inventário de Sintomas de Dor Neuropática; DP: Desvio Padrão
} 
Tabela 21 - Avaliação dos doentes através do BPI e da EVA de acordo com o grupo e com o período em médias e desvio padrão, e resultados dos testes de associação (p) $(\mathrm{N}=29)$

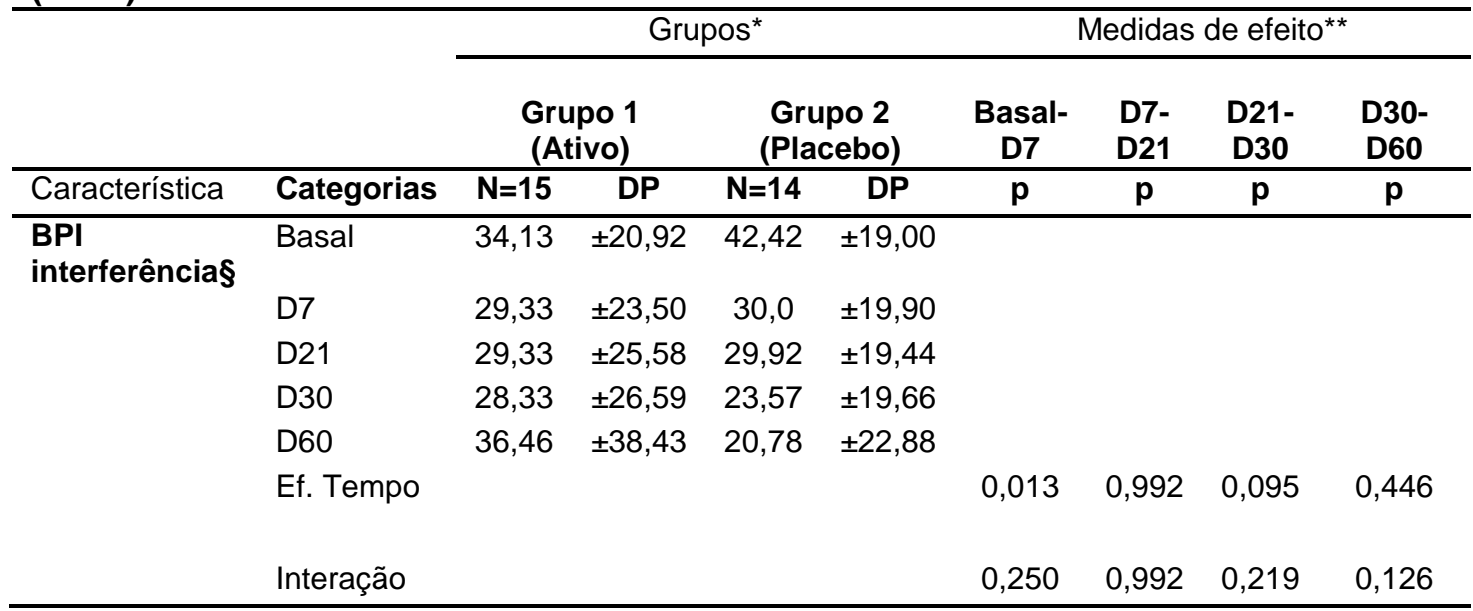

\footnotetext{
**Análise de variância para medidas repetidas; §presença apenas de efeito de tempo,

*** presença de interação. BPI: Inventário breve de dor; EVA: Escala Visual Analógica;

DP: Desvio Padrão.
}

Tabela 22. Avaliação dos doentes através do Questionário de Dor McGill de acordo com o grupo e com o período em médias e desvio padrão, e resultados dos testes de associação (p) ( $\mathrm{N}=29)$.

\begin{tabular}{|c|c|c|c|c|c|c|c|c|c|}
\hline \multirow[b]{3}{*}{ Característica } & \multirow[b]{3}{*}{ Categorias } & \multicolumn{4}{|c|}{ Grupos* } & \multicolumn{4}{|c|}{ Medidas de efeito** } \\
\hline & & \multicolumn{2}{|c|}{$\begin{array}{c}\text { Grupo } 1 \\
\text { (Ativo) }\end{array}$} & \multicolumn{2}{|c|}{$\begin{array}{l}\text { Grupo } 2 \\
\text { (Placebo) }\end{array}$} & \multirow{2}{*}{$\begin{array}{c}\text { Basal- } \\
\text { D7 } \\
p\end{array}$} & \multirow{2}{*}{$\begin{array}{c}\text { D7- } \\
\text { D21 } \\
p\end{array}$} & \multirow{2}{*}{$\begin{array}{c}\text { D21- } \\
\text { D30 } \\
p\end{array}$} & \multirow{2}{*}{$\begin{array}{c}\begin{array}{c}\text { D30- } \\
\text { D60 }\end{array} \\
p\end{array}$} \\
\hline & & $\mathrm{N}=15$ & DP & $\mathrm{N}=14$ & DP & & & & \\
\hline \multirow{7}{*}{$\begin{array}{l}\text { McGill- } \\
\text { sensitivo§ }\end{array}$} & Basal & 17,86 & $\pm 3,87$ & 12,85 & $\pm 7,53$ & & & & \\
\hline & D7 & 15,73 & $\pm 7,37$ & 13,64 & $\pm 7,57$ & & & & \\
\hline & D21 & 16,40 & $\pm 7,62$ & 13,92 & $\pm 6,92$ & & & & \\
\hline & D30 & 15,26 & $\pm 8,89$ & 11,50 & $\pm 7,14$ & & & & \\
\hline & D60 & 14,26 & $\pm 7,68$ & 9,50 & $\pm 5,93$ & & & & \\
\hline & Ef. Tempo & & & & & 0,521 & 0,612 & 0,058 & 0,143 \\
\hline & Interação & & & & & 0,170 & 0,839 & 0,479 & 0,619 \\
\hline \multirow{7}{*}{$\begin{array}{l}\text { McGill- } \\
\text { afetivo }\end{array}$} & Basal & 10,66 & $\pm 2,54$ & 7,64 & $\pm 4,10$ & & & & \\
\hline & D7 & 8,06 & $\pm 3,59$ & 8,00 & $\pm 3,90$ & & & & \\
\hline & D21 & 7,33 & $\pm 4,04$ & 8,07 & $\pm 4,00$ & & & & \\
\hline & D30 & 7,40 & $\pm 4,40$ & 7,14 & $\pm 3,89$ & & & & \\
\hline & D60 & 7,46 & $\pm 4,47$ & 5,57 & $\pm 3,20$ & & & & \\
\hline & Ef. Tempo & & & & & 0,124 & 0,507 & 0,137 & 0,341 \\
\hline & Interação & & & & & $0,031^{* * *}$ & 0,421 & 0,088 & 0,300 \\
\hline \multirow[t]{6}{*}{ McGill-total§ } & Basal & 27,93 & $\pm 5,75$ & 20,50 & $\pm 10,65$ & & & & \\
\hline & D7 & 23,80 & $\pm 10,07$ & 21,64 & $\pm 10,87$ & & & & \\
\hline & D21 & 23,73 & $\pm 10,68$ & 22,00 & $\pm 10,04$ & & & & \\
\hline & D30 & 22,66 & $\pm 13,12$ & 18,64 & $\pm 10,09$ & & & & \\
\hline & D60 & 21,73 & $\pm 11,58$ & 15,07 & $\pm 8,76$ & & & & \\
\hline & $\begin{array}{l}\text { Ef. Tempo } \\
\text { Interação }\end{array}$ & & & & & $\begin{array}{l}0,283 \\
0,064\end{array}$ & $\begin{array}{l}0,887 \\
0,821\end{array}$ & $\begin{array}{l}0,037 \\
0,265\end{array}$ & $\begin{array}{l}0,192 \\
0,440\end{array}$ \\
\hline
\end{tabular}

\footnotetext{
${ }^{* *}$ Análise de variância para medidas repetidas; §presença apenas de efeito de tempo.

*** presença de interação. McGill: Questionário de dor McGill. DP: Desvio Padrão.
} 
Tabela 23 - Avaliação dos doentes através do Questionário de qualidade de vida SF36 de acordo com o grupo e com o período em médias e desvio padrão, e resultados dos testes de associação (p) ( $N=29)$

\begin{tabular}{|c|c|c|c|c|c|c|c|c|c|}
\hline \multirow[b]{3}{*}{ Característica } & \multirow[b]{3}{*}{ Categorias } & \multicolumn{4}{|c|}{ Grupos* $^{*}$} & \multicolumn{4}{|c|}{ Medidas de efeito** } \\
\hline & & \multicolumn{2}{|c|}{$\begin{array}{c}\text { Grupo 1 } \\
\text { (Ativo) }\end{array}$} & \multicolumn{2}{|c|}{$\begin{array}{c}\text { Grupo } 2 \\
\text { (Placebo) }\end{array}$} & \multirow{2}{*}{$\begin{array}{c}\begin{array}{c}\text { Basal- } \\
\text { D7 }\end{array} \\
\mathbf{p} \\
\end{array}$} & \multirow{2}{*}{$\begin{array}{c}\text { D7- } \\
\text { D21 } \\
p\end{array}$} & \multirow{2}{*}{$\begin{array}{c}\text { D21- } \\
\text { D30 } \\
p\end{array}$} & \multirow{2}{*}{$\begin{array}{c}\text { D30- } \\
\text { D60 } \\
p\end{array}$} \\
\hline & & $\mathrm{N}=15$ & DP & $\mathrm{N}=14$ & DP & & & & \\
\hline \multirow{7}{*}{$\begin{array}{l}\text { SF36 - capacidade } \\
\text { funcional }\end{array}$} & Basal & 61,33 & $\pm 28,43$ & 66,42 & $\pm 27,56$ & & & & \\
\hline & D7 & 61,66 & $\pm 25,40$ & 60,00 & $\pm 24,88$ & & & & \\
\hline & D21 & 62,66 & $\pm 25,02$ & 70,71 & $\pm 23,27$ & & & & \\
\hline & D30 & 64,00 & $\pm 28,85$ & 65,00 & $\pm 27,59$ & & & & \\
\hline & $\mathrm{D} 60$ & 63,00 & $\pm 31,83$ & 75,71 & $\pm 28,81$ & & & & \\
\hline & Ef. Tempo & & & & & 0,267 & 0,063 & 0,254 & 0,120 \\
\hline & Interação & & & & & 0,219 & 0,120 & 0,072 & 0,063 \\
\hline \multirow[t]{7}{*}{ SF36 - aspecto físico } & Basal & 43,33 & $\pm 43,77$ & 39,38 & $\pm 45,69$ & & & & \\
\hline & D7 & 43,33 & $\pm 42,74$ & 26,78 & $\pm 31,72$ & & & & \\
\hline & D21 & 36,66 & $\pm 41,04$ & 32,14 & $\pm 43,22$ & & & & \\
\hline & D30 & 64,00 & $\pm 28,85$ & 65,00 & $\pm 27,59$ & & & & \\
\hline & $\mathrm{D} 60$ & 36,66 & $\pm 48,05$ & 26,78 & $\pm 38,56$ & & & & \\
\hline & Ef. Tempo & & & & & 0,451 & 0,932 & 0,560 & 0,214 \\
\hline & Interação & & & & & 0,451 & 0,433 & 0,560 & 0,965 \\
\hline \multirow{7}{*}{$\begin{array}{l}\text { SF36 - estado geral de } \\
\text { saúde§ }\end{array}$} & Basal & 60,40 & $\pm 13,36$ & 59,78 & $\pm 11,28$ & & & & \\
\hline & D7 & 61,86 & $\pm 11,28$ & 61,35 & $\pm 12,20$ & & & & \\
\hline & D21 & 63,20 & $\pm 13,36$ & 64,35 & $\pm 12,15$ & & & & \\
\hline & D30 & 53,93 & $\pm 27,16$ & 44,14 & $\pm 24,09$ & & & & \\
\hline & $\mathrm{D} 60$ & 53,13 & $\pm 28,62$ & 47,21 & $\pm 25,31$ & & & & \\
\hline & Ef. Tempo & & & & & 0,104 & 0,398 & 0,006 & 0,687 \\
\hline & Interação & & & & & 0,954 & 0,743 & 0,278 & 0,494 \\
\hline \multirow[t]{7}{*}{ SF36 - vitalidade } & Basal & 48,66 & $\pm 24,74$ & 43,92 & $\pm 21,04$ & & & & \\
\hline & D7 & 48,66 & $\pm 29,18$ & 46,42 & $\pm 19,35$ & & & & \\
\hline & $\mathrm{D} 21$ & 51,66 & $\pm 26,29$ & 47,50 & $\pm 18,57$ & & & & \\
\hline & D30 & 54,33 & $\pm 26,71$ & 47,50 & $\pm 18,57$ & & & & \\
\hline & $\mathrm{D} 60$ & 49,00 & $\pm 26,13$ & 50,35 & $\pm 16,46$ & & & & \\
\hline & Ef. Tempo & & & & & 0,589 & 0,529 & 0,543 & 0,548 \\
\hline & Interação & & & & & 0,589 & 0,765 & 0,795 & 0,233 \\
\hline \multirow{7}{*}{$\begin{array}{l}\text { SF36 - Aspectos } \\
\text { sociais }\end{array}$} & Basal & 57,50 & $\pm 32,32$ & 42,85 & $\pm 32,41$ & & & & \\
\hline & D7 & 62,50 & $\pm 32,38$ & 60,71 & $\pm 25,87$ & & & & \\
\hline & $\mathrm{D} 21$ & 59,16 & $\pm 36,43$ & 58,03 & $\pm 23,31$ & & & & \\
\hline & D30 & 57,50 & $\pm 35,91$ & 58,92 & $\pm 22,16$ & & & & \\
\hline & $\mathrm{D} 60$ & 60,83 & $\pm 34,99$ & 58,92 & $\pm 27,48$ & & & & \\
\hline & Ef. Tempo & & & & & 0,110 & 0,390 & 0,916 & 0,642 \\
\hline & Interação & & & & & 0,135 & 0,925 & 0,729 & 0,642 \\
\hline \multirow{7}{*}{$\begin{array}{l}\text { SF36 -Aspectos } \\
\text { emocionais }\end{array}$} & Basal & 31,11 & $\pm 40,75$ & 33,33 & $\pm 39,22$ & & & & \\
\hline & D7 & 44,44 & $\pm 44,83$ & 33,33 & $\pm 41,34$ & & & & \\
\hline & D21 & 46,66 & $\pm 37,37$ & 40,47 & $\pm 43,71$ & & & & \\
\hline & D30 & 44,44 & $\pm 43,03$ & 30,95 & $\pm 40,22$ & & & & \\
\hline & $\mathrm{D} 60$ & 40,00 & $\pm 45,77$ & 35,71 & $\pm 42,29$ & & & & \\
\hline & Ef. Tempo & & & & & 0,382 & 0,489 & 0,417 & 0,976 \\
\hline & Interação & & & & & 0,382 & 0,715 & 0,613 & 0,389 \\
\hline
\end{tabular}

${ }^{* *}$ Análise de variância para medidas repetidas; §presença apenas de efeito de tempo. Sf-36: Inventário de qualidade de vida. DP: Desvio Padrão. 
Tabela 24 - Avaliação dos doentes através do DN4 de acordo com o grupo e com o período em médias e desvio padrão, e resultados dos testes de associação ( $p$ ) ( $N=29$ )

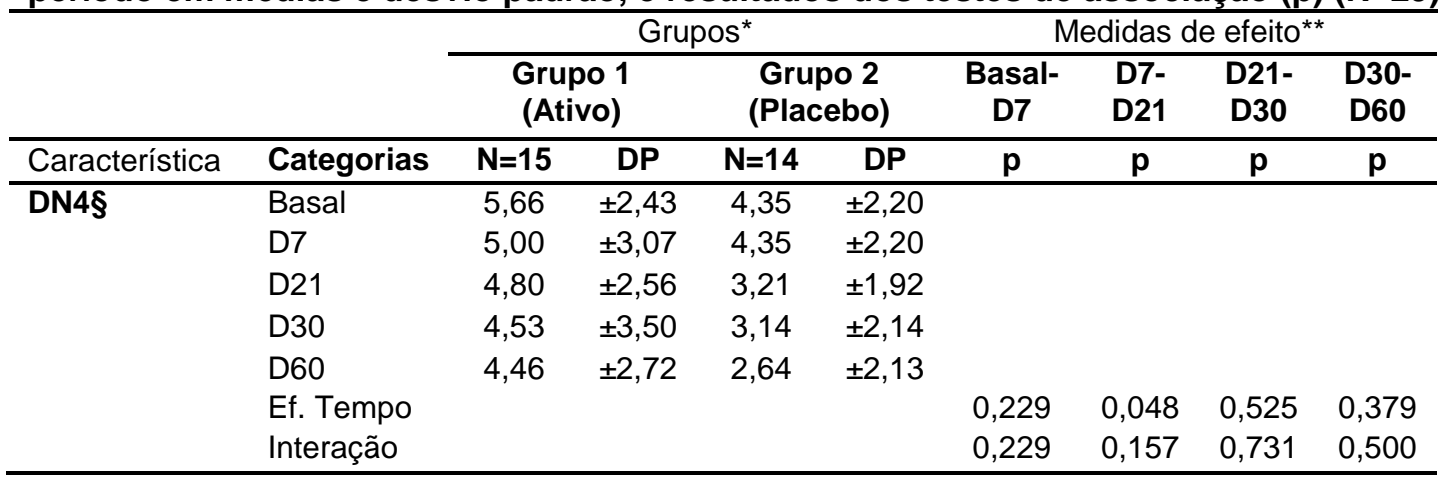

${ }^{* *}$ Análise de variância para medidas repetidas; §presença apenas de efeito de tempo. DN4: questionário para diagnóstico de dor neuropática; DP: Desvio Padrão. 


\section{Anexo 1: Aprovação do Comitê de Ética em Pesquisa do Hospital das Clínicas da Faculdade de Medicina da Universidade de São Paulo.}

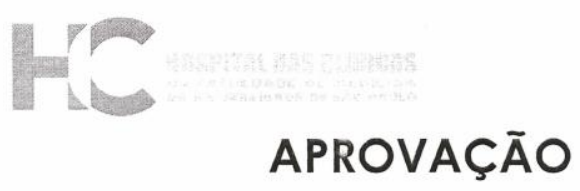

A Comissão de Ética para Análise de Projetos de Pesquisa CAPPesq da Diretoria Clínica do Hospital das Clínicas da Faculdade de Medicina da Universidade de São Paulo, em sessão de 02/02/2011. APrOVOU o Protocolo de Pesquisa n 0674/10, intitulado: "AVALIAÇĀO DE DOENTES COM DOR FACIAL ATÍPICA (DFA) ATRAVÉS DE MAGNETOENCEFALOGRAFIA, TRATADOS COM ESTIMULAÇĀO MAGNÉtICA TRANSCRANIANA (EMT)" apresentado pelo Departamento de NEUROLOGIA, inclusive o Termo de Consentimento Livre e Esclarecido.

Cabe ao pesquisador elaborar e apresentar à CAPPesq, os relatórios parciais e final sobre a pesquisa (Resolução do Conselho Nacional de Saúde $n^{\circ} 196$, de 10/10/1996, inciso IX.2, letra "c").

Pesquisador (a) Responsável: Prof.Dr. Manoel Jacobsen Teixeira / Profa.Dra.Silvia R.D. T. de Siqueira

Pesquisador (a) Executante: Ricardo Galhardoni

CAPPesq, 04 de Fevereiro de 2011

$$
\begin{gathered}
\text { PROF. DR. EUCLIDES AYRES DE CASTILHO } \\
\text { Coordenador } \\
\text { Comissão de Ética para Análise de } \\
\text { Projetos de Pesquisa - CAPPesq }
\end{gathered}
$$

Comissăo de Ética para Análise de Projetos de Pesquisa do HCFMUSP da Diretoria Clinica do Hospital das Clinicas da Faculdade de Medicina da Universidade de São Paulo Rua Ovídio Pires de Campos, 225, $5^{\circ}$ andar - CEP 05403010 - São Paulo - SP Fone: 01130696442 Fax: 01130696492

e-mail: cappesq@hcnet.usp.br 


\title{
Anexo 2: Registro do protocolo de estudo na plataforma ClinicalTrials.gov
}

\author{
ClinicalTrials.gov \\ Protocol Registration System
}

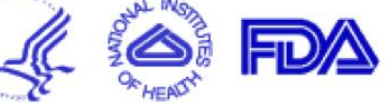

Protocol Registration Receipt

$04 / 24 / 2014$

Assessment and Treatment Patients With Atypical Facial Pain Trough Repetitive

Transcranial Magnetic Stimulation (EMTr-AFP)

This study has been completed.

\begin{tabular}{|r|l|}
\hline Sponsor: & University of Sao Paulo \\
\hline Collaborators: & \\
\hline $\begin{array}{r}\text { Information provided by } \\
\text { (Responsible Party): }\end{array}$ & Ricardo Galhardoni, University of Sao Paulo \\
\hline ClinicalTrials.gov Identifier: & NCT01746355 \\
\hline
\end{tabular}

\section{Purpose}

Atypical Facial Pain is a chronic condition and presents controversies during diagnostic and treatment, between specialist not have consensus about the pathophysiology. It is possible consider this entitie a potential neuropathic cause without pathological signs. It knows the repetitive transcranial magnetic presents good results in the treatment of chronic pain coditions. The aim this study is evaluated the thresholds and excitability cortical in patients with AFP and verify the patterns of improvement in pain for patients undergoing rTMS compared to controls, as well as the therapeutic response to neuromodulation procedures. This enroll 20 patients with DAF and 20 controls treated with rTMS (5 sessions) and evaluated through questionnaires (EDOF clinical record, McGill Pain Questionnaire, Visual Analogue Scale, Inventory of symptoms of neuropathic pain, neuropathic pain DN4 questionnaire, SF -36, brief pain inventory). At the end of the data will be statistically analyzed and expressed as mean and standard deviation, and analyzed by Student's $t$ test, analysis of variance (ANOVA), Tukey-Kramer and Pearson correlation. The level of significance is $5 \%$. The results will be published in journals indexed in the area both nationally and internationally and presented at conferences and scientific meetings.

\begin{tabular}{|l|l|l|}
\hline Condition & Intervention & Phase \\
\hline $\begin{array}{l}\text { Atypical Facial Pain or Atypical } \\
\text { Odontalgia }\end{array}$ & Procedure/Surgery: rTMS & N/A \\
\hline
\end{tabular}




\title{
Anexo 3: Termo de Consentimento Livre e Esclarecido
}

\section{HOSPITAL DAS CLÍNICAS DA FACULDADE DE MEDICINA DA UNIVERSIDADE DE SÃO PAULO- HCFMUSP TERMO DE CONSENTIMENTO LIVRE E ESCLARECIDO}

\author{
DADOS DE IDENTIFICAÇÃO DO SUJEITO DA PESQUISA OU RESPONSÁVEL LEGAL \\ 1. NOME: \\ DE IDENTIDADE № : \\ SEXO : .M $\square \mathrm{F}$ \\ DATA NASCIMENTO: \\ ENDEREÇO: $\quad$ № APTO: BAIRRO:

CIDADE: CEP:___. TELEFONE: DDD ( ) \\ 2. RESPONSÁVEL LEGAL \\ NATUREZA (grau de parentesco, tutor, curador etc.) \\ DOCUMENTO DE IDENTIDADE : \\ ENDEREÇO: \\ SEXO: $M \square F \square$ DATA NASCIMENTO.: \\ CIDADE: \\ № \\ APTO \\ BAIRRO: \\ SOBRE A PESQUISA \\ 1. TÍTULO DO PROTOCOLO DE PESQUISA: \\ Avaliação e tratamento de doentes com dor facial atípica (DFA) através de estimulação \\ magnética transcraniana repetitiva (EMTr)
}

2. PESQUISADOR : Manoel Jacobsen Teixeira

CARGO/FUNÇÃO: Professor titular de Neurocirurgia, Departamento de Neurologia. INSCRIÇÃO CONSELHO REGIONAL № 17968

UNIDADE DO HCFMUSP: Equipe de Dor Orofacial e Liga de Dor, Divisão de Neurologia, Instituto Central. Pesquisador executante: Ricardo Galhardoni

\section{AVALIAÇÃO DO RISCO DA PESQUISA:}

$\begin{array}{lr}\text { RISCO MÍNIMO } & \text { RISCO MÉDIO } \\ \text { RISCO BAIXO } & \text { RISCO MAIOR }\end{array}$

4. DURAÇÃO DA PESQUISA : 2 anos.

1 - Desenho do estudo e objetivo(s):

$\mathrm{Sr}(\mathrm{a})$, a dor que o senhor(a) apresenta ainda tem causa pouco conhecida e é de difícil tratamento. Estamos realizando um estudo para a avaliação do tratamento de sua doença através da estimulação magnética transcraniana, um método que tem sido utilizado para outras dores com bons resultados.

2 - Descrição dos procedimentos que serão realizados, com seus propósitos e identificação dos que forem experimentais e não rotineiros;

$\mathrm{Sr}(\mathrm{a})$, utilizaremos questionários e faremos uma avaliação através de um aparelho das áreas do cérebro afetadas pela dor, daí procederemos a estimulação magnética transcraniana, (que poderá ser ativa ou placebo, ou seja, bobina ligada ou desligada), que acontecerá através de uma bobina colocada próxima a sua cabeça. Esse procedimento não é novo e também não é invasivo, ou seja, todos os exames e o tratamento são aplicados sobre a pele e couro cabeludo e seus efeitos são bem conhecidos.

3 - Relação dos procedimentos rotineiros e como são realizados:

Inicialmente, serão aplicados os questionários para o (a) Sr (a) responder. Para avaliação da excitabilidade cortical e para o tratamento da sua dor, o (a) $\mathrm{Sr}$ (a). será colocado (a) em uma cadeira confortável onde uma bobina será posicionada perto de sua cabeça. O procedimento em geral é indolor e o (a) Sr (a) deve apenas ouvir alguns barulhos que correspondem à atividade do aparelho. Após o tratamento, o (a) Sr (a) será novamente avaliado (a) através de questionários.

4 - Descrição dos desconfortos e riscos esperados nos procedimentos dos itens 2 e 3;

Em alguns pacientes, pode ocorrer alguns sintomas durante a estimulação como: dor de cabeça, ruídos nos ouvidos, dor no pescoço, dor nos dentes e excepcionalmente crises convulsivas na presença de estimulação de altas freqüências. $\mathrm{O}$ (a) $\operatorname{Sr}($ a) será devidamente orientado e tratado caso algum desconforto ocorra. 
5 - Benefícios para o participante

Este estudo permitirá verificar a eficácia desse tratamento em pessoas que tem o tipo de dor facial que o (a) Sr (a) tem, além de ajudar no entendimento dos mecanismos envolvidos nessas dores como, por exemplo, quais as áreas do cérebro afetadas por essa doença. O (a) Sr (a) continuará sendo tratado pelo grupo de dor durante e após a estimulação na divisão de neurologia.

6 - Relação de procedimentos alternativos que possam ser vantajosos, pelos quais o paciente pode optar:

O paciente pode optar a qualquer momento a interromper o estudo, e também estarei sempre disponível para esclarecimentos de dúvidas e questões que surjam antes, durante, ou depois da pesquisa.

7 - Garantia de acesso: em qualquer etapa do estudo, você terá acesso aos profissionais responsáveis pela pesquisa para esclarecimento de eventuais dúvidas. Os principais investigadores são os Prof. Dr. Manoel Jacobsen Teixeira, Profa. Dra. Silvia R.D.T. de Siqueira, Dr. Daniel Ciampi de Andrade e o pesquisador executante é Ricardo Galhardoni, que poderão ser encontrados no Instituto

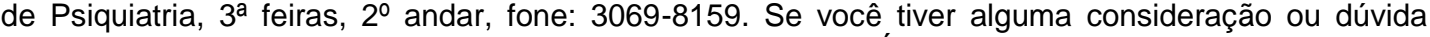
sobre a ética da pesquisa, entre em contato com o Comitê de Ética em Pesquisa (CEP) - Rua Ovídio Pires de Campos, 225 - 5 andar - tel: 3069-6442 ramais 16, 17, 18 ou 20, FAX: 3069-6442 ramal 26 - E-mail: cappesq@hcnet.usp.br

8 - É garantida a liberdade da retirada de consentimento a qualquer momento e deixar de participar do estudo, sem qualquer prejuízo à continuidade de seu tratamento na Instituição;

09 - Direito de confidencialidade - As informações obtidas serão analisadas em conjunto com outros pacientes, não sendo divulgada a identificação de nenhum paciente;

10 - Direito de ser mantido atualizado sobre os resultados parciais das pesquisas, quando em estudos abertos, ou de resultados que sejam do conhecimento dos pesquisadores;

11 - Despesas e compensações: não há despesas pessoais para o participante em qualquer fase do estudo, incluindo exames e consultas. Também não há compensação financeira relacionada à sua participação. Se existir qualquer despesa adicional, ela será absorvida pelo orçamento da pesquisa.

12 - Em caso de dano pessoal, diretamente causado pelos procedimentos ou tratamentos propostos neste estudo (nexo causal comprovado), o participante tem direito a tratamento médico na Instituição, bem como às indenizações legalmente estabelecidas.

13 - Compromisso do pesquisador de utilizar os dados e o material coletado somente para esta pesquisa.

Acredito ter sido suficientemente informado a respeito das informações que li ou que foram lidas para mim, descrevendo o estudo Avaliação e tratamento de doentes com dor facial atípica (DFA) através de estimulação magnética transcraniana repetitiva (EMTr).

Eu discuti com o Prof. Dr. Manoel Jacobsen Teixeira / Ricardo Galhardoni sobre a minha decisão em participar nesse estudo. Ficaram claros para mim quais são os propósitos do estudo, os procedimentos a serem realizados, seus desconfortos e riscos, as garantias de confidencialidade e de esclarecimentos permanentes. Ficou claro também que minha participação é isenta de despesas e que tenho garantia do acesso a tratamento hospitalar quando necessário. Concordo voluntariamente em participar deste estudo e poderei retirar o meu consentimento a qualquer momento, antes ou durante o mesmo, sem penalidades ou prejuízo ou perda de qualquer benefício que eu possa ter adquirido, ou no meu atendimento neste Serviço.

Assinatura do paciente/representante legal

Assinatura da testemunha

Data $\frac{1}{1}$

para casos de pacientes menores de 18 anos, analfabetos, semi-analfabetos ou portadores de deficiência auditiva ou visual.

(Somente para o responsável do projeto)

Declaro que obtive de forma apropriada e voluntária o Consentimento Livre e Esclarecido deste paciente ou representante legal para a participação neste estudo.

Assinatura do responsável pelo estudo

Data

11




\section{Anexo 4: Critérios de Inclusão e exclusão do grupo controle e doentes}

\begin{tabular}{|c|c|c|}
\hline \multicolumn{3}{|l|}{ Critérios de Inclusão } \\
\hline & Sim & Não \\
\hline $\begin{array}{l}\text { Preenche os critérios de DFA, segundo a Classificação de Cafaleias. } \\
\text { 13.18.4 Persistent idiopathic facial pain Previously used term: Atypical facial } \\
\text { pain } \\
\text { Description: } \\
\text { Persistent facial pain that does not have the charac- } \\
\text { teristics of the cranial neuralgias and is not attributed to another disorder. } \\
\text { Diagnostic criteria: } \\
\text { A. Pain in the face, present daily and persisting for all or most of the day, } \\
\text { fulfilling criteria B and C } \\
\text { B. Pain is confined at onset to a limited area on one side of the face }{ }^{1} \text {, and is } \\
\text { deep and poorly localized. } \\
\text { C .Pain is not associated with sensory loss or other physical signs. } \\
\text { D. Investigations including X-ray of face and jaws do not demonstrate any } \\
\text { relevant abnormality. }\end{array}$ & & \\
\hline 2) Assinar o termo de consentimento livre-esclarecido (TCLE) & & \\
\hline 3) Idade superior a 18 anos & & \\
\hline 4) Se em tratamento, não ter descontinuado no último mês & & \\
\hline \multicolumn{3}{|l|}{ Critérios de Exclusão } \\
\hline & Sim & Não \\
\hline $\begin{array}{l}\text { Encefalopatias focais ou generalizadas (tumores, acidentes vásculo- } \\
\text { cerebrais, meningite, encefalite) (GEORGE; BELMAKER 2000) }\end{array}$ & & \\
\hline $\begin{array}{l}\text { Trauma craniano, epilepsia não tratada, indivíduos com parentes de } \\
\text { primeiro grau com epilepsia idiopática, }\end{array}$ & & \\
\hline $\begin{array}{l}\text { Pacientes com uso inicial recente de medicações que reduzem o limiar } \\
\text { convulsivo (antidepressivos tricíclicos e antipsicóticos), }\end{array}$ & & \\
\hline $\begin{array}{l}\text { Indivíduos em uso crônico de álcool ou drogas epileptogênicas (ex. } \\
\text { cocaína) }\end{array}$ & & \\
\hline $\begin{array}{l}\text { Cardiopatias graves, clipes neurocirúrgicos, marca-passo cardíaco, } \\
\text { aumento de pressão intracraniana (risco de sequelas após convulsão) }\end{array}$ & & \\
\hline Mulheres grávidas ou amamentando & & \\
\hline
\end{tabular}

Note:

1. Pain at onset is commonly in the nasolabial fold or side of the chin, and may spread to the upper or lower jaw or a wider area of the face and neck.

Comments:

Pain may be initiated by surgery or injury to the face, teeth or gums but persists without any demonstrable local cause. Facial pain around the ear or temple may precede the detection of an ipsilateral lung carcinoma causing referred pain by invasion of the vagus nerve. The term atypical odontalgia has been applied to a continuous pain in the teeth or in a tooth socket after extraction in the absence of any identifiable dental cause. 


\section{Anexo 5: Ficha Clínica da Equipe de Dor Orofacial (EDOF)}

Ficha Clínica da Equipe de Dor Orofacial / ATM Divisão de Odontologia do Hospital das Clínicas - FMUSP

Nome:

$\mathrm{RGHC}$ Sexo:

Dentista: Tel: Data:

Clínica: Origem:

Idade: Altura: Peso: Profissão:

Cor: ( ) B ( ) N ( ) Outra Estado civil: O paciente está:

( ) só ( ) acompanhado por ( ) cadeira de rodas

I. ANAMNESE - CARACTERÍSTICAS DA DOR

1. QP (Qual é a sua queixa?)

2. Há quanto tempo você tem essa dor? ( ) Dias ( ) Meses ( ) Anos 3. Periodicidade:

( ) Diária... ( ) 2-3 X sem ( ) Sem ( ) Quinz ( ) Mensal.

4. Periodo do dia que tem dor: ( ) M ( ) T () N ( ) Indiferente $\underline{5}$ : Como ela aparece? ( ) Espontânea

( ) Provocada: Como?

() segs ( ) mins ( ) horas () dias ( ) Outro $\underline{6}$ Quanto tempo dura a sua dor?

7. Tipo (caracteristica) da dor: ( ) Pontada ( ) Peso ( ) Queimor ( ) Choque ( ) Latejante ( ) Contínua ( ) Outro

8. Intensidade da dor: ( ) fraca ( ) moderada ( ) forte $\underline{9}$. Nota de 0 a 10:

10. Essa dor te acorda durante o sono? ( ) N ( ) S

11. Período do dia em que a dor é pior: ( ) M ( ) T ( ) N ( ) sono ( ) indiferente

( ) outro:

13. Sabe o que iniciou a sua dor? ( ) N ( ) S - Como?

14. O que piora a sua

dor?

15. O que acalma a sua

dor?

16. Tratamentos realizados para a dor e melhora

$(\mathrm{M}, \mathrm{PM}, \mathrm{SM})$

17. Possui o hábito de morder: ( ) língua ( ) bochecha ( ) lábios ( ) objetos:

18. Você mastiga do lado: ( ) D ( ) E ( ) na frente ( ) bilateral $\underline{19}$. - Você acha que sua mastigação é: ( ) boa ( ) ruim ( ) péssima ( ) não sabe ( ) causa dor - Onde?

20. Ao acordar sente alguma dor em seu corpo? ( )N ( )S:- ( )rosto ( )ouvido ( )cabeça ()dentes ( ) pescoço ( ) corpo ( ) Outro 
21 - Sente o rosto cansado com frequência: ( ) N ( ) S - ( ) ao acordar ( ) ao mastigar ( ) ao falar ( ) ao sorrir ( ) outro

22 - Sabe se range os dentes: ( ) N ( ) não sabe ( ) S ( ) à noite ( ) de dia

Quem disse?

23 - Sente ruídos na ( ) face ( ) cabeça ( ) N () S - Lado? Quando? ( ) AB ( ) fala

( ) mastiga ( ) outro

24 - Tem dor provocada por algum movimento da boca? ()N ()S - ( ) AB ( ) Protrusão ( ) later. D ( ) Later.E ( ) outro

25 - Tem dor de ouvido? ( ) N ( ) S: Lado? ()D ()E Passou pelo médico (ORL)? ()N ()S - O que ele disse?

26 - Tem dor de cabeça? ( ) N ( ) S: Onde?

27 - Passou pelo médico (Neuro)? ( ) N () S - o que ele disse ou receitou?

28 - Tem dor no corpo: ( ) N ( ) S: Onde?

29- Passou pelo médico? ( ) N ( ) S- Qual? O que ele disse ou receitou?

30 - Teve algum acidente, cirurgia ou doença grave? ( ) N ( ) S - Qual, como e onde afetou seu corpo?

31. Mostre onde é a sua dor.

E as dores do seu corpo.

() $\mathrm{D}$

( ) Bilateral

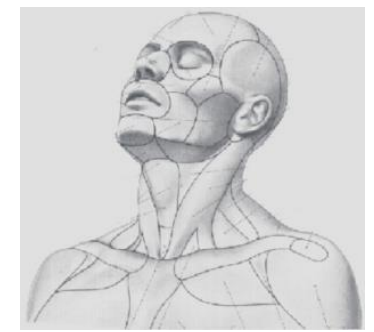

III. ANTECEDENTES MÉDICOS PESSOAIS:
( ) Artrite reumatóide
( ) Asma
( ) Amigdalite
( ) Derrame (AVC)
( ) Fibromialgia
( ) Sinusite
( ) Úlcera
( ) Gastrite
( ) Rinite alérgica
( ) Coração
( ) Infecções
( ) Enxaqueca

( ) Herpes zoster (cobreiro) ( ) Parkison

( ) Rebordo

( ) Dentes
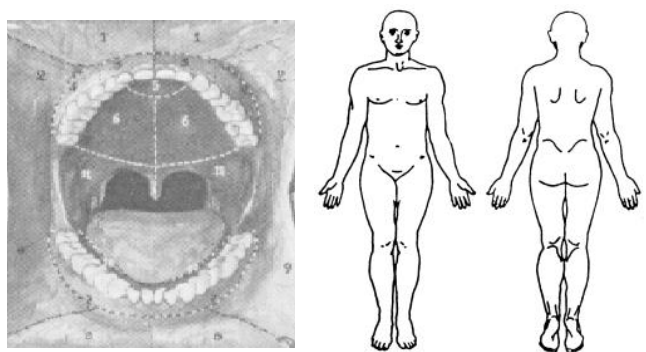

32. Tratou-se de alguma destas doenças:

( ) Bronquite

( ) Pressão alta (HAS)

( ) Doença renal (rins)

( ) Outra:
( ) Hepatite

( ) Diabete

( ) Depressão 
33. Está em tratamento médico atual? ( ) N ( ) S Doenças que tem e remédios que usa:

\section{ASPECTOS PSICOLÓGICOS}

34. Comportamento durante a consulta:

\section{EXAME FÍSICO:}

35. Face: ( ) Assimetria facial ( ) Prognatismo ( ) Laterognatismo:-D E Hipertrofia: ( ) Masseter ( )Temporal - ( ) D ( ) E

36. Pele da Face:

\section{Linfonodos:}

\section{Mucosa oral:}

39. Lingua:

40. Alterações neurológicas:

41. Periodonto:

42. Dentes:

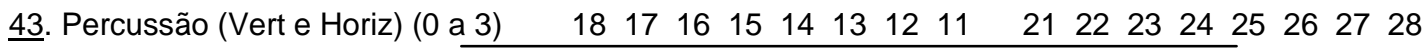

44. Ausências dentárias (/) $\quad$\begin{tabular}{lllllllllllllllll}
\cline { 2 - 3 } & 47 & 46 & 45 & 44 & 43 & 42 & 41 & 31 & 32 & 33 & 34 & 35 & 36 & 37 & 38
\end{tabular}

45. Interferências oclusais:

46. Mordida aberta: ( ) S ( ) N 느. Mordida cruzada: ( ) Ant ( ) Post- ( ) D ( ) E

48. Sobremordida profunda: ( ) N ( ) S - ( ) a ( ) b ( ) c

49. Desgastes dentários: ( ) N ( ) S - ( ) incisais ( ) 1/3 incisal ( ) 1/3 médio ( ) 1/3 cervical

50. Angle: ( )CI I ( )Cl II ( )Cl III

51. Desdentado Total: ( ) Duplo ( ) Sup ( ) Inf 52. PPR: ( ) N ( ) S-Qual:

53. Perda de DV:( ) N ( ) S - ___ mm 54. Tempo de uso de PT:

55. Tempo da PT atual:

56. Tempo de uso da PPR:

57. Movimentos mandibulares: AB: ___ $\mathrm{mm}-($ ) sem dor ( ) com dor - local:

$\mathbf{P}: \ldots+\ldots \mathrm{mm}-()$ sem dor ()com dor - local: ___ _ _ LD: ___ mm - ()sem dor ()com dor local: _ _ _ _ LE:___ $\mathrm{mm}-($ ) sem dor ( ) com dor - local:

Media: DLMf: _ _ mm ()E ()D DLMa: _

Linha 
58. Ruídos na ATM: ( ) Ausentes ( ) POP ( ) Crepitação ( ) D ( ) E Estalo D- ( ) IA ( ) MA ( ) FA ( ) IF ( ) MF ( ) FF Estalo E - ( ) IA ( ) MA ( ) FA ( ) IF ( ) MF ( ) FF

59. Palpação da ATM e dos músculos da mastigação e do pescoço:

\begin{tabular}{|c|c|c|c|}
\hline ATM ou Músculos & Dir & Esq & Obs. \\
\hline \multicolumn{4}{|l|}{ ATM - polo lateral } \\
\hline \multicolumn{4}{|l|}{$\begin{array}{l}\text { ATM - polo } \\
\text { posterior }\end{array}$} \\
\hline \multicolumn{4}{|l|}{ Masseter Inferior } \\
\hline \multicolumn{4}{|l|}{ Masseter Médio } \\
\hline \multicolumn{4}{|l|}{ Masseter Superior } \\
\hline \multicolumn{4}{|l|}{$\begin{array}{l}\text { Masseter Intra- } \\
\text { Oral }\end{array}$} \\
\hline \multicolumn{4}{|l|}{ Temporal Anterior } \\
\hline \multicolumn{4}{|l|}{ Temporal Médio } \\
\hline \multicolumn{4}{|l|}{$\begin{array}{l}\text { Temporal } \\
\text { Posterior }\end{array}$} \\
\hline \multicolumn{4}{|l|}{$\begin{array}{l}\text { Temporal Intra- } \\
\text { Oral }\end{array}$} \\
\hline \multicolumn{4}{|l|}{ Digástrico anterior } \\
\hline \multicolumn{4}{|l|}{$\begin{array}{l}\text { Digástrico } \\
\text { posterior }\end{array}$} \\
\hline \multicolumn{4}{|l|}{ ECM superior } \\
\hline \multicolumn{4}{|l|}{ ECM médio } \\
\hline \multicolumn{4}{|l|}{ ECM inferior } \\
\hline \multicolumn{4}{|l|}{ Esplênio cervical } \\
\hline \multicolumn{4}{|l|}{$\begin{array}{l}\text { Esplênio da } \\
\text { cabeça }\end{array}$} \\
\hline \multicolumn{4}{|l|}{ Suboccipitais } \\
\hline \multicolumn{4}{|l|}{ Trapézio ombro } \\
\hline Trapézio pescoço & & & \\
\hline
\end{tabular}

60. Movimentos cervicais dolorosos? ( ) N ( ) S- rotação D ( ) rotação E ( ) extensão () flexão obs.

61. Rx, exames ou interconsultas solicitadas:

62. Hipótese Diagnóstica para a dor

(CID):

63. Diagnósticos secundários (CID):

64. Diagnóstico final

(dor):

65. Tratamento sugerido para a

dor:

66. Reabilitações sugeridas: 
Anexo 6: Questionário de dor McGill resumido

\begin{tabular}{|l|l|l|l|l|}
\hline \multicolumn{5}{|l|}{ Questionário de dor McGill - resumido } \\
\hline & Ausente & Fraca & Moderada & Forte \\
\hline Pulsátil & & & & \\
\hline Em choques & & & & \\
\hline Em facadas & & & & \\
\hline Viva & & & & \\
\hline Como câimbras & & & & \\
\hline Que corrói & & & & \\
\hline Quente, que queima & & & & \\
\hline Surda & & & & \\
\hline Pesada & & & & \\
\hline Sensível ao contato & & & & \\
\hline Que rasga & & & & \\
\hline Cansativa, exaustiva & & & & \\
\hline Adoecedora & & & & \\
\hline Amedrontadora & & & \\
\hline Cruel, que pune & & & \\
\hline
\end{tabular}

B. Intensidade atual da dor
0 - nenhuma dor
1- leve
2- desagradável
3- esgotante
4- horrível
5- excruciante 


\section{Anexo 7: Inventário de Sintomas de Dor Neuropática (NPSI)}

\section{Inventário de Sintomas de Dor Neuropática (NPSI)}

Você tem sofrido de dor devido a lesão ou doença do sistema nervoso. Esta dor pode ser de diversos tipos. Você pode ter dor espontânea, ex: dor na ausência de qualquer estímulo, que pode ser duradoura ou ocorrer em ataques breves. Você pode também ter dor provocada ou aumentada por leve toque, pressão ou contato com o frio na área dolorosa. Você pode sentir um ou mais tipos de dor. Este questionário foi desenvolvido para ajudar o seu médico a melhor avaliar e tratar diferentes tipos de dor que possa sentir.

Nós queremos saber se você sente dor espontânea, isto é dor sem qualquer estímulo. Para cada uma das seguintes questões, por favor selecione o número que melhor descreve a sua gravidade média da dor espontânea durante as últimas 24 horas. Selecione o número 0 se você não sentiu tal dor (circule o número apenas).

Q1. A sua dor dá a sensação de queimação?

$\begin{array}{lllllllllllll}\text { Não queima } & 0 & 1 & 2 & 3 & 4 & 5 & 6 & 7 & 8 & 9 & 10 & \text { A pior }\end{array}$

Queimadura

Imaginável

Q2. A sua dor dá a sensação de apertar?

$\begin{array}{llllllllllll}\text { Não aperta } & 0 & 1 & 2 & 3 & 4 & 5 & 6 & 7 & 8 & 9 & 10 \\ \text { Aperta }\end{array}$

O pior

Imaginável

Q3. A sua dor dá a sensação de pressão?

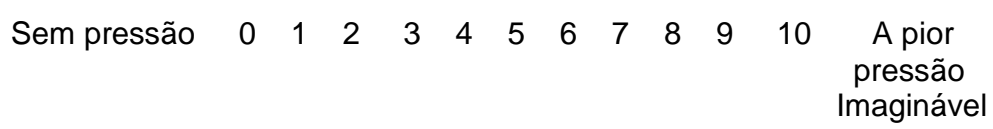

Q4. Durante as últimas 24 horas, a sua dor espontânea tem estado presente:

Selecione a resposta que melhor descreve o seu caso

$\begin{array}{ll}\text { Permanentemente } & (\text { ) } \\ \text { Entre } 8 \text { e } 12 \mathrm{~h} & (\text { ) } \\ \text { Entre } 4 \text { e } 7 \mathrm{~h} & (\text { ) } \\ \text { Entre } 1 \text { e } 3 \mathrm{~h} & (\text { ) } \\ \text { menos que } 1 \mathrm{~h} & \end{array}$

Nós queremos saber se você teve ataques breves de dor. Para cada uma das seguintes questões, por favor, selecione o número que melhor descreve a gravidade média dos seus ataques de dor durante as últimas 24 horas. Selecione o número 0 se você não sentiu tal dor (circule um número apenas).

Q5 A sua dor dá a sensação de choque elétrico?

$\begin{array}{lllllllllllll}\text { Sem choque elétrico } & 0 & 1 & 2 & 3 & 4 & 5 & 6 & 7 & 8 & 9 & 10 & \text { O pior }\end{array}$

Choque elétrico

imaginável

Q6 A sua dor dá a sensação de apunhalar?

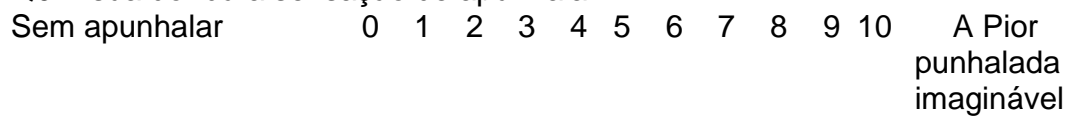

Q7. Durante as últimas 24 horas, quantos destes ataques de dor teve? Selecione a resposta que melhor descreve o seu caso.

Mais de 20

Entre 11 e 20

Entre 6 e10

Entre 1 e 5

Sem ataque de dor

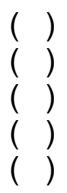


Nós queremos saber se você sente dor provocada ou aumentada por leve toque, pressão, contacto com frio na área onde dói. Para cada das seguintes questões, por favor selecione o número que melhor descreve a gravidade media da dor provocada durante as últimas 24 horas. Selecione 0 número 0 se você não sentiu tal dor (circule um número apenas).

Q8. A sua dor é provocada ou aumentada por um leve toque na área dolorosa?

Sem dor $0 \begin{array}{llllllllllll}0 & 1 & 2 & 3 & 4 & 5 & 6 & 7 & 8 & 9 & 10 & \text { A pior dor }\end{array}$ Imaginável

Q9. A sua dor é provocada ou aumentada por pressão na área dolorosa?

Sem dor $\begin{array}{lllllllllllll}0 & 1 & 2 & 3 & 4 & 5 & 6 & 7 & 8 & 9 & 10 & \text { A pior dor }\end{array}$ Imaginável

Q10. A sua dor é provocada ou aumentada por contacto com algo frio na área dolorosa?

$\begin{array}{lllllllllllll}\text { Sem dor } & 0 & 1 & 2 & 3 & 4 & 5 & 6 & 7 & 8 & 9 & 10 & \begin{array}{c}\text { Pior dor } \\ \text { Imaginável }\end{array}\end{array}$

Nós queremos saber se você sente sensações anormais na zona onde dói. Para cada uma das seguintes questões, por favor selecione o número que melhor descreve a gravidade média das sensações anormais durante as últimas 24 horas. Selecione o número 0 se você não sentiu tal dor (circule um número apenas).

Q11. Sente alfinetadas e agulhadas?

Sem alfinetes nem agulhas $\begin{array}{lllllllllll}0 & 1 & 2 & 3 & 4 & 5 & 6 & 7 & 8 & 9 & 10\end{array}$

Os piores alfinetadas e agulhadas

I Imagináveis

Q12. Sente Dormente?

$\begin{array}{lllllllllllll}\text { Sem Dormência } & 0 & 1 & 2 & 3 & 4 & 5 & 6 & 7 & 8 & 9 & 10 & \text { O mais dormente }\end{array}$ Imaginável 


\section{Anexo 8: Questionário de Dor Neuropática 4 (DN-4)}

Por favor, nas quatro perguntas abaixo, complete o questionário marcando uma resposta para cada número:

\section{ENTREVISTA DO PACIENTE}

Questão 1: A sua dor tem uma ou mais das seguintes características?

1 - Queimação

2 - Sensação de frio dolorosa

3 - Choque elétrico
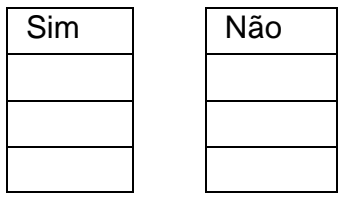

Questão 2: Há presença de um ou mais dos seguintes sintomas na mesma área da sua dor?

4 - Formigamento

5 - Alfinetada e Agulhada

6 - Adormecimento

7 - Coceira
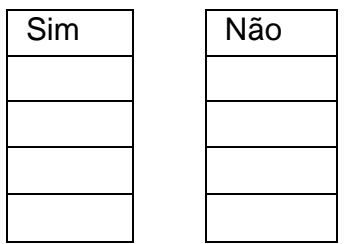

\section{EXAME DO PACIENTE}

Questão 3. A dor está localizada numa área onde o exame físico pode revelar uma ou mais das seguintes caraterísticas?

8- Hipoestesia ao toque

9- Hipoestesia a picada de agulha

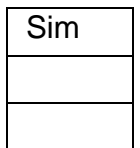

Não

Questão 4. Na área dolorosa, a dor pode ser causada ou aumentada por:

10 - Escovação 


\section{Anexo 9: Questionário de Qualidade de Vida SF-36}

1- Em geral você diria que sua saúde é:

\begin{tabular}{|c|c|c|c|c|}
\hline Excelente & Muito Boa & Boa & Ruim & Muito Ruim \\
\hline 1 & 2 & 3 & 4 & 5 \\
\hline
\end{tabular}

2- Comparada há um ano atrás, como você se classificaria sua idade em geral, agora?

\begin{tabular}{|c|c|c|c|c|}
\hline Muito Melhor & Um Pouco Melhor & Quase a Mesma & Um Pouco Pior & Muito Pior \\
\hline 1 & 2 & 3 & 4 & 5 \\
\hline
\end{tabular}

3- Os seguintes itens são sobre atividades que você poderia fazer atualmente durante um dia comum. Devido à sua saúde, você teria dificuldade para fazer estas atividades? Neste caso, quando?

\begin{tabular}{|l|c|c|c|}
\hline \multicolumn{1}{|c|}{ Atividades } & $\begin{array}{c}\text { Sim, dificulta } \\
\text { muito }\end{array}$ & $\begin{array}{c}\text { Sim, dificulta } \\
\text { um pouco }\end{array}$ & $\begin{array}{c}\text { Não, não } \\
\text { dificulta de } \\
\text { modo algum }\end{array}$ \\
\hline $\begin{array}{l}\text { a) Atividades Rigorosas, que exigem } \\
\text { muito esforço, tais como correr, } \\
\text { levantar objetos pesados, participar } \\
\text { em esportes árduos. }\end{array}$ & 1 & 2 & 3 \\
\hline $\begin{array}{l}\text { b) Atividades moderadas, tais como } \\
\text { mover uma mesa, passar aspirador de } \\
\text { pó, jogar bola, varrer a casa. }\end{array}$ & 1 & 2 & 3 \\
\hline c) Levantar ou carregar mantimentos & 1 & 2 & 3 \\
\hline d) Subir vários lances de escada & 1 & 2 & 3 \\
\hline e) Subir um lance de escada & 1 & 2 & 3 \\
\hline f) Curvar-se, ajoelhar-se ou dobrar-se & 1 & 2 & 3 \\
\hline g) Andar mais de 1 quilômetro & 1 & 2 & 3 \\
\hline h) Andar vários quarteirões & 1 & 2 & 3 \\
\hline i) Andar um quarteirão & 1 & 2 & 3 \\
\hline j) Tomar banho ou vestir-se & 1 & 2 & 3 \\
\hline
\end{tabular}

4- Durante as últimas 4 semanas, você teve algum dos seguintes problemas com seu trabalho ou com alguma atividade regular, como consequência de sua saúde física?

\begin{tabular}{|l|c|c|}
\hline & Sim & Não \\
\hline $\begin{array}{l}\text { a) Você diminui a quantidade de tempo que se dedicava ao seu } \\
\text { trabalho ou a outras atividades? }\end{array}$ & 1 & 2 \\
\hline b) Realizou menos tarefas do que você gostaria? & 1 & 2 \\
\hline c) Esteve limitado no seu tipo de trabalho ou a outras atividades. & 1 & 2 \\
\hline $\begin{array}{l}\text { d) Teve dificuldade de fazer seu trabalho ou outras atividades (p. ex. } \\
\text { necessitou de um esforço extra). }\end{array}$ & 1 & 2 \\
\hline
\end{tabular}


5- Durante as últimas 4 semanas, você teve algum dos seguintes problemas com seu trabalho ou outra atividade regular diária, como consequência de algum problema emocional (como se sentir deprimido ou ansioso)?

\begin{tabular}{|c|c|c|}
\hline & Sim & Não \\
\hline $\begin{array}{l}\text { a) Você diminui a quantidade de tempo que se dedicava ao seu } \\
\text { trabalho ou a outras atividades? }\end{array}$ & 1 & 2 \\
\hline b) Realizou menos tarefas do que você gostaria? & 1 & 2 \\
\hline $\begin{array}{l}\text { c) Não realizou ou fez qualquer das atividades com tanto cuidado } \\
\text { como geralmente faz. }\end{array}$ & 1 & 2 \\
\hline
\end{tabular}

6- Durante as últimas 4 semanas, de que maneira sua saúde física ou problemas emocionais interferiram nas suas atividades sociais normais, em relação à família, amigos ou em grupo?

\begin{tabular}{|c|c|c|c|c|}
\hline De forma nenhuma & Ligeiramente & Moderadamente & Bastante & Extremamente \\
\hline 1 & 2 & 3 & 4 & 5 \\
\hline
\end{tabular}

7- Quanta dor no corpo você teve durante as últimas 4 semanas?

\begin{tabular}{|c|c|c|c|c|c|}
\hline Nenhuma & Muito leve & Leve & Moderada & Grave & Muito grave \\
\hline 1 & 2 & 3 & 4 & 5 & 6 \\
\hline
\end{tabular}

8- Durante as últimas 4 semanas, quanto a dor interferiu com seu trabalho normal (incluindo o trabalho dentro de casa)?

\begin{tabular}{|c|c|c|c|c|}
\hline De maneira alguma & Um pouco & Moderadamente & Bastante & Extremamente \\
\hline 1 & 2 & 3 & 4 & 5 \\
\hline
\end{tabular}

9- Estas questões são sobre como você se sente e como tudo tem acontecido com você durante as últimas 4 semanas. Para cada questão, por favor, dê a resposta que mais se aproxime da maneira como você se sente em relação à esse período.

\begin{tabular}{|l|c|c|c|c|c|r|}
\hline & $\begin{array}{c}\text { Todo } \\
\text { Tempo }\end{array}$ & $\begin{array}{c}\text { A maior } \\
\text { parte do } \\
\text { tempo }\end{array}$ & $\begin{array}{c}\text { Uma boa } \\
\text { parte do } \\
\text { tempo }\end{array}$ & $\begin{array}{c}\text { Alguma } \\
\text { parte } \\
\text { do } \\
\text { tempo }\end{array}$ & $\begin{array}{c}\text { Uma } \\
\text { pequena } \\
\text { parte do } \\
\text { tempo }\end{array}$ & $\begin{array}{c}\text { u } \\
\text { cmpon } \\
\text { a }\end{array}$ \\
\hline $\begin{array}{l}\text { a) Quanto tempo você } \\
\text { tem se sentindo cheio } \\
\text { de vigor, de vontade, } \\
\text { de força? }\end{array}$ & 1 & 2 & 3 & 5 & 6 \\
\hline $\begin{array}{l}\text { b) Quanto tempo você } \\
\text { tem se sentido uma } \\
\text { pessoa muito } \\
\text { nervosa? }\end{array}$ & 1 & 2 & 3 & 4 & 5 & 6 \\
\hline $\begin{array}{l}\text { c) Quanto tempo você } \\
\text { tem se sentido tão } \\
\text { deprimido que nada }\end{array}$ & 1 & 2 & 3 & 4 & 5 & 6 \\
\hline
\end{tabular}




\begin{tabular}{|l|c|c|c|c|c|c|}
\hline pode anima-lo? & & & & & & \\
\hline $\begin{array}{l}\text { d) Quanto tempo você } \\
\text { tem se sentido calmo } \\
\text { ou tranquilo? }\end{array}$ & 1 & 2 & 3 & 4 & 5 & 6 \\
\hline $\begin{array}{l}\text { e) Quanto tempo você } \\
\text { tem se sentido com } \\
\text { muita energia? }\end{array}$ & 1 & 2 & 3 & 4 & 5 & 6 \\
\hline $\begin{array}{l}\text { f) Quanto tempo você } \\
\text { tem se sentido } \\
\text { desanimado ou } \\
\text { abatido? }\end{array}$ & 1 & 2 & 3 & 4 & 5 & 6 \\
\hline $\begin{array}{l}\text { g) Quanto tempo você } \\
\text { tem se sentido } \\
\text { esgotado? }\end{array}$ & 1 & 2 & 3 & 4 & 5 & 6 \\
\hline $\begin{array}{l}\text { h) Quanto tempo você } \\
\text { tem se sentido uma } \\
\text { pessoa feliz? }\end{array}$ & 1 & 2 & 3 & 4 & 5 & 6 \\
\hline $\begin{array}{l}\text { i) Quanto tempo você } \\
\text { tem se sentido } \\
\text { cansado? }\end{array}$ & 1 & 2 & 3 & 4 & 5 & 6 \\
\hline
\end{tabular}

10- Durante as últimas 4 semanas, por quanto tempo a sua saúde física ou problemas emocionais interferiram com as suas atividades sociais (como visitar amigos, parentes etc.)?

\begin{tabular}{|c|c|c|c|c|}
\hline $\begin{array}{c}\text { Todo } \\
\text { Tempo }\end{array}$ & $\begin{array}{c}\text { A maior parte do } \\
\text { tempo }\end{array}$ & $\begin{array}{c}\text { Alguma parte do } \\
\text { tempo }\end{array}$ & $\begin{array}{c}\text { Uma pequena } \\
\text { parte do tempo }\end{array}$ & $\begin{array}{c}\text { Nenhuma } \\
\text { parte do } \\
\text { tempo }\end{array}$ \\
\hline 1 & 2 & 3 & 4 & 5 \\
\hline
\end{tabular}

11- O quanto verdadeiro ou falso é cada uma das afirmações abaixo para você?

\begin{tabular}{|c|c|c|c|c|c|}
\hline & $\begin{array}{l}\text { Definitivamente } \\
\text { verdadeiro }\end{array}$ & $\begin{array}{l}\text { A maioria } \\
\text { das vezes } \\
\text { verdadeiro }\end{array}$ & $\begin{array}{l}\text { Não } \\
\text { sei }\end{array}$ & $\begin{array}{c}\text { A } \\
\text { maioria } \\
\text { das } \\
\text { vezes } \\
\text { falso }\end{array}$ & $\begin{array}{c}\text { De } \\
\text { fin } \\
\text { itiv } \\
\text { a- } \\
\text { me } \\
\text { nt } \\
\text { e } \\
\text { fal } \\
\text { so }\end{array}$ \\
\hline $\begin{array}{l}\text { a) Eu costumo adoecer } \\
\text { um pouco mais } \\
\text { facilmente que as outras } \\
\text { pessoas }\end{array}$ & 1 & 2 & 3 & 4 & 5 \\
\hline $\begin{array}{l}\text { b) Eu sou tão saudável } \\
\text { quanto qualquer pessoa } \\
\text { que eu conheço }\end{array}$ & 1 & 2 & 3 & 4 & 5 \\
\hline $\begin{array}{l}\text { c) Eu acho que a minha } \\
\text { saúde vai piorar }\end{array}$ & 1 & 2 & 3 & 4 & 5 \\
\hline $\begin{array}{l}\text { d) Minha saúde é } \\
\text { excelente }\end{array}$ & 1 & 2 & 3 & 4 & 5 \\
\hline
\end{tabular}


Anexo 10: Inventário Breve de Dor

\section{INVENTÁRIO BREVE DE DOR}

1) Durante a vida, a maioria das pessoas apresenta dor de vez em quando (dor de cabeça, dor de dente, etc.). Você teve hoje, dor diferente dessas?

1.Sim $\square$ 2.Não $\square$

2) Marque sobre o diagrama um $X$ nas áreas onde você sente dor, e onde a dor é mais intensa.

Frente

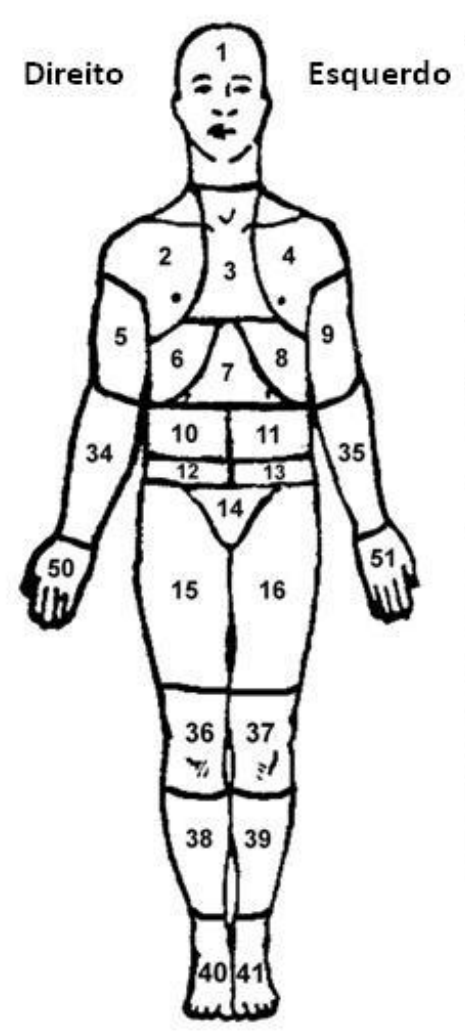

Costas

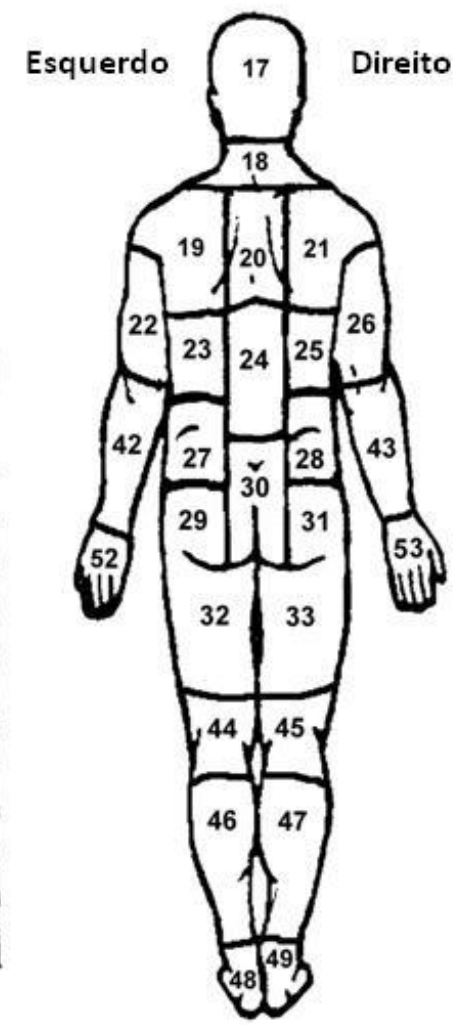

3) Circule o número que melhor descreve a pior dor que você sentiu nas últimas 24 horas.

Sem dor \begin{tabular}{|lllllllllll|l}
0 & 1 & 2 & 3 & 4 & 5 & 6 & 7 & 8 & 9 & 10 & Pior dor possível
\end{tabular}

4) Circule o número que melhor descreve a dor mais fraca que você sentiu nas últimas 24 horas.

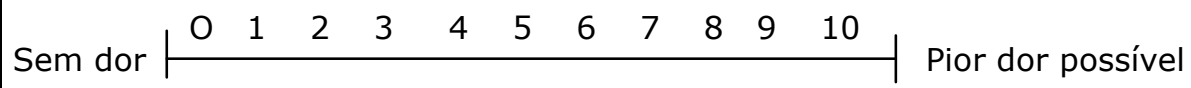


5) Circule o número que melhor descreve a média da sua dor.

Sem dor $\mid$\begin{tabular}{lllllllllll|l|}
0 & 1 & 2 & 3 & 4 & 5 & 6 & 7 & 8 & 9 & 10 \\
\hline
\end{tabular}

6) Circule o número que mostra quanta dor você está sentindo agora (neste momento).

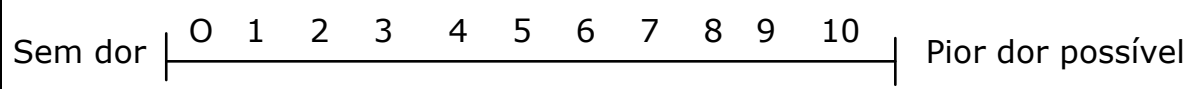

\begin{tabular}{|l|l|l|}
\hline \multicolumn{3}{|c|}{ 7) Quais tratamentos ou medicações você está recebendo para dor? } \\
\hline Nome & Dose/ Frequência & Data de Início \\
\hline & & \\
\hline & & \\
\hline & & \\
\hline & & \\
\hline & & \\
\hline & & \\
\hline & & \\
\hline
\end{tabular}

8) Nas últimas 24 horas, qual a intensidade da melhora proporcionada pelos tratamentos ou medicações que você está usando?

Circule o percentual que melhor representa o alívio que você obteve.

Sem alívio \begin{tabular}{lllllllll|}
$0 \%$ & $10 \%$ & $20 \%$ & $30 \%$ & $40 \%$ & $50 \%$ & $60 \%$ & $70 \%$ & alívio
\end{tabular} completo

9) Circule o número que melhor descreve como, nas últimas 24 horas, a dor interferiu na sua:

Atividade geral

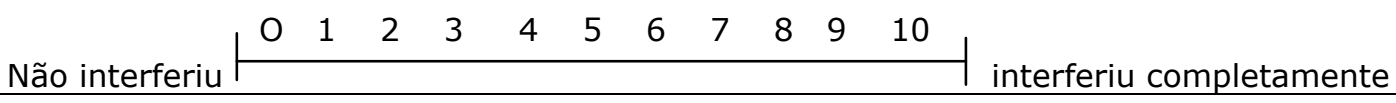

Humor

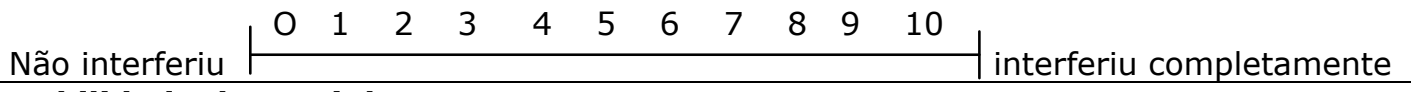

Habilidade de caminhar

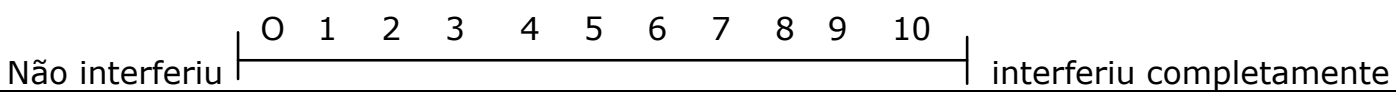

Trabalho

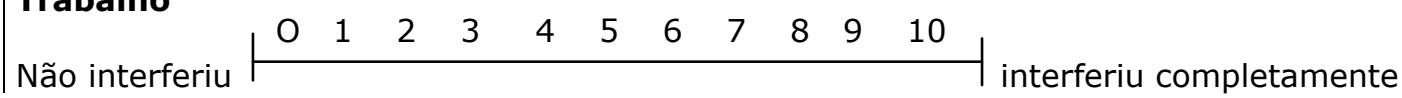




\begin{tabular}{|c|c|c|c|c|c|c|c|c|c|c|c|c|c|}
\hline \multicolumn{14}{|c|}{ Relacionamento com outras pessoas } \\
\hline \multirow[b]{2}{*}{ Não interferiu } & $\mathrm{O}$ & 1 & 2 & 3 & 4 & 5 & & & 7 & 8 & 9 & 10 & \multirow[b]{2}{*}{ interferiu completamente } \\
\hline & & & & & & & & & & & & & \\
\hline \multicolumn{14}{|l|}{ Sono } \\
\hline & $\mathrm{O}$ & 1 & 2 & 3 & 4 & & & & 7 & 8 & 9 & 10 & \\
\hline \multirow{2}{*}{\multicolumn{14}{|c|}{$\begin{array}{l}\text { Não interferiu } \\
\text { Habilidade para apreciar a vida }\end{array}$}} \\
\hline & & & & & & & & & & & & & \\
\hline & $\mathrm{O}$ & 1 & 2 & 3 & 4 & 5 & & & 7 & 8 & 9 & 10 & \multirow[b]{2}{*}{ interferiu completamente } \\
\hline Não interferiu & & & & & & & & & & & & & \\
\hline
\end{tabular}

